\begin{abstract}
CHANG, HUAN-YU. Damage Visualization of Scattered Ultrasonic Wavefield via Integrated High-speed Camera System (Under the direction of Dr. Fuh-Gwo Yuan).

The ultimate goal for this research is to see through the structure in order to visualize the invisible hidden damages in the structures via camera, so that the damage image can be shown by simply taking a "video" on the structure. Traditionally, for full-field scanning by laser Doppler vibrometer (LDV), the wavefield needs to be reconstructed by measuring each point sequentially. The camera enable measuring all the points at the same time and replacing the current LDV system. However the performance of current high-speed camera is still limited, so for the development of damage imaging algorithm, it needs to be tested with the current laser system.
\end{abstract}

As the first step in the development of image processing algorithm, a fully non-contact laser scanning system was developed to detect and visualize a barely visible impact damage (BVID) in a honeycomb composite panel. A zero-lag cross correlation (ZLCC) imaging condition was employed for damage imaging. ZLCC results were in very good agreement with ultrasonic Cscan and X-ray CT scan results. However, ZLCC was the damage imaging technique based on frequency-wavenumber filtering which requires multiple dimensional Fourier transform, am algorithm for "video" was needed without complex transform and could be operated in time domain.

Imaging a damage using the phase of wavefield "video" was then developed and applied to a stiffened curved composite sandwich panel for visualization of BVID with the laser system. The wavefield "video" was reconstructed through the measurement by LDV. Newly generated wavenumbers from the geometry/material discontinuities caused by either the stiffener or the impact damage can be detected by observing the change of wavenumber. The instantaneous 
wavenumber derived via Riesz transform (RT) and its difference can be shown and highlighted using the developed imaging condition, named wavenumber index (WI), which is different to other techniques implemented in the frequency-wavenumber domain by the use of complex wavenumber filtering and wave mode decomposition. Since WI is the phase-based imaging technique instead of conventional intensity-based technique for the wavefield "video", this technique is robust in that the impact damages located in the vicinity of geometry/material discontinuities can yield consistent damage image resolution with high sensitivity even for wave propagating from the direction across the stiffener. The BVID of the composite structure becomes therefore "visible".

The WI technique was then further applied and integrated with a high-speed camera scanning system, and it successfully visualized the hidden damage through reconstructed scattered wavefield. By employing a sample interleaving and an image stitching technique, the data requisition rate for the high-speed camera was enhanced nearly 250 times in order to overcome the current limited performance due to inadequate on both spatial resolution and temporal resolution, for guided wavefield measurement. Further, many experimental parameters, e.g., specimen design, field of view, speckle size and signal frequency, as well as the camera system were carefully designed, integrated and optimized to enable capture of propagating guided waves excited by a piezo actuator on a surface of the structure. As such the in-plane wave displacements could be maximized and extracted using digital image correlation (DIC).

A very compliant HDPE plate with an added mass for mimicking damage was chosen for the proof of concept. The first fundamental $S H$ and $S_{0}$ guided wave modes under continuous wave were successfully detected by the camera and extracted by DIC, and the disturbance at the damage region was clearly observed in the reconstructed scattered wavefield. The WI technique was then 
improved and applied for the wavefield reconstructed via high-speed DIC. In this dissertation, the hidden damage was successfully visualized through camera for the very first time. 
(C) Copyright 2019 by Huan-Yu Chang

All Rights Reserved 
Damage Visualization of Scattered Ultrasonic Wavefield via Integrated High-speed Camera System

by

\begin{abstract}
A dissertation submitted to the Graduate Faculty of
North Carolina State University

in partial fulfillment of the requirements for the degree of

Doctor of Philosophy
\end{abstract}

Mechanical Engineering

Raleigh, North Carolina

2019

\title{
APPROVED BY:
}

Dr. Fuh-Gwo Yuan

Committee Chair

Dr. Larry Silverberg
Dr. Chih-Hao Chang

Dr. Xiangwu Zhang 


\section{DEDICATION}

To my parents who always support me

and

all those who inspired me 


\section{BIOGRAPHY}

Huan-Yu (Tony) Chang is a goal-oriented mechanical engineer and passionate research scientist, who is specialized in automotive engineering, composite structure, finite element analysis (FEA) and nondestructive inspection (NDI).

Tony was born on February 18, 1987, in Taipei, Taiwan. He received his B.S. in 2009 and M.S in 2011, in the Department of Mechanical Engineering at National Taiwan University (NTU). During his college time, he joined the solar car team at NTU, which was later transformed to Formosun Advanced Power Research Center (FAPRC), and started his career path toward an excellent mechanical engineer in automotive industry. He participated the projects building a fuel-cell electric scooter and an electric vehicle (EV), and he was responsible for the entire chassis and part of the structure. In the last year of his grad school, he became the leader of the entire team to finalize the EV project while he was initiating a new project for a new conceptual EV chassis.

After the one-year mandatory military service, Tony started working at Hua-Chuang Automobile Information Technical Center (HAITEC), which is the research and development center of a local automotive company in Taiwan, as a suspension engineer responsible for wheels, tires and the twist beam for the rear suspension.

After leaving HAITEC, he started pursuing his Ph.D. degree in the Department of Mechanical and Aerospace Engineering in Raleigh, NC in 2014. He spent most of his Ph.D. life in working on his dissertation research as a research assistant in the National Institute of Aerospace (NIA) located at Hampton, Virginia. He earned his degree in September 2019. 


\section{ACKNOWLEDGMENTS}

I would like to express my sincere gratitude to my advisor Dr. Fuh-Gwo Yuan for his patient guidance, profound insight, professional attitude and dedication towards researches. Thank you for your support, countless advice and timely response to all my questions. I would also like to thank my committee members: Dr. Chi-Hao Chang, Dr. Larry Silverberg, and Dr. Xiangwu Zhang for your time, advises and helps in completing my dissertation. I also want to thank Dr. Chau-Wai Wong to serve as my substitute committee member in my final defense at a very short notice. Thanks to National Institute of Aerospace for all supports in financial of scholarship, courses, and life helps. I spend meaningful time in National Institute of Aerospace, Virginia.

I would also like to thank the members, graduates and friends in our research group at North Carolina State University: Dr. Jiaze He, Chao Wan, Karthik, and Sakib; Labmate in NIA, Dr. CheYuan Chang, Dr. Tyler Hudson, Dr. Yu-Sheng Chang, Dongwon Lee, and Abel Fong. Thank you for all the helpful supports I had with you.

Additionally, I would like to thank all the friends that I have met in Virginia, I could have not continued this long and lonely journey if it wasn't for all your company.

My gratitude also goes to my dear parents and sister, who have unconditionally loved and supported me. You are always supporting and encouraging me with your best wishes. 


\section{TABLE OF CONTENTS}

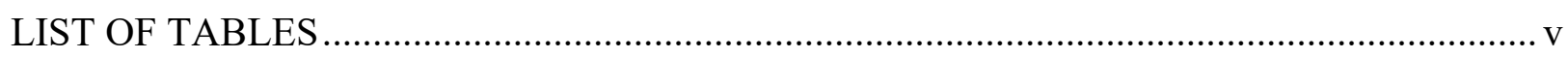

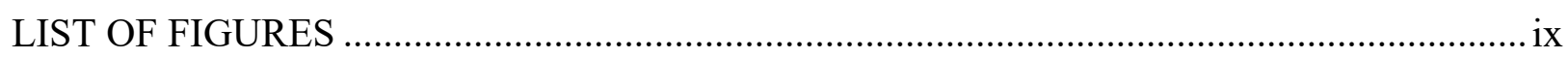

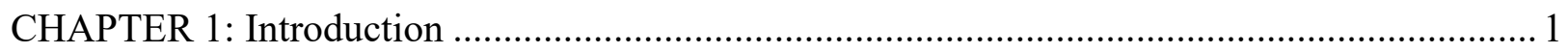

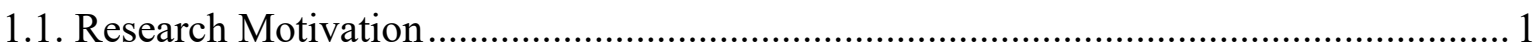

1.2. Motion Magnification ......................................................................................... 4

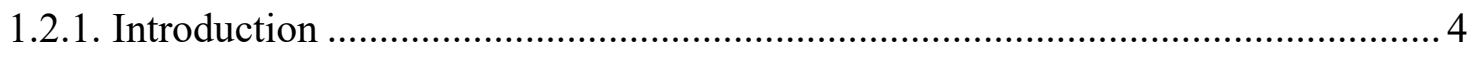

1.2.2. Eularian Motion Magnification ....................................................................... 5

1.2.3. Complex steerable pyramids phase-based motion magnification ........................ 7

1.2.4. Riesz pyramid phase-based motion magnification.............................................. 10

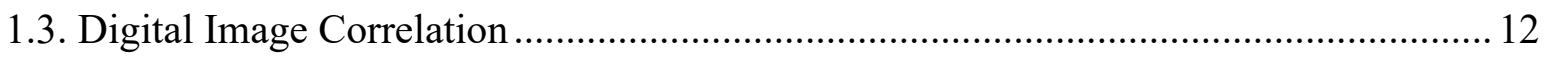

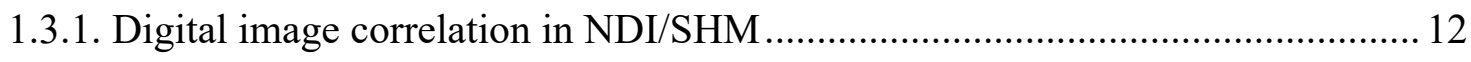

1.3.2. Cross-correlation in displacement extraction ................................................... 16

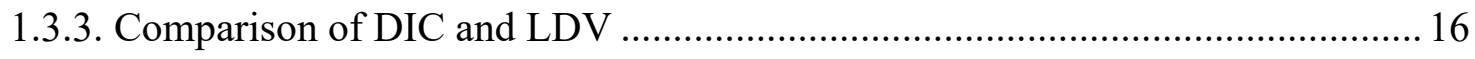

1.4. Guided Waves in Damage Detection for Composites ................................................ 18

1.5. Objectives and Dissertation Outline .................................................................... 24

CHAPTER 2: Image Processing via Hilbert and Reisz transform ................................................ 32

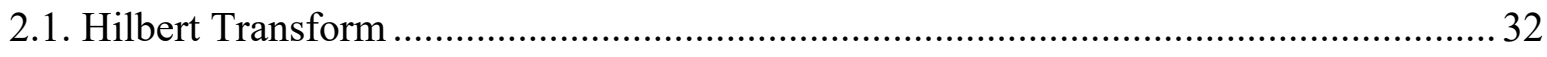

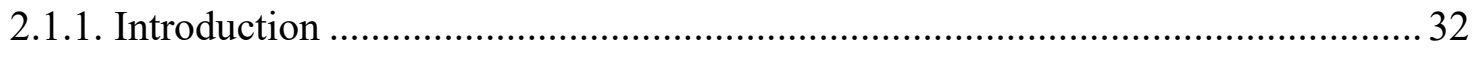

2.1.2. Definition of Hilbert transform in time domain .................................................. 39

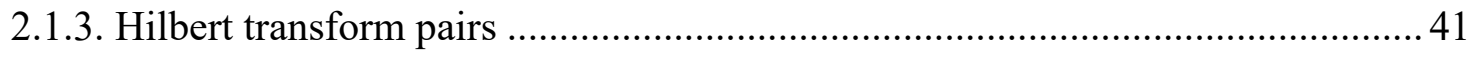

2.1.4. Definition of Hilbert transform in frequency domain ......................................... 43

2.1.5. Analytic signals and instantaneous parameters ............................................... 47 
2.2. Hilbert Spectral Analysis ....................................................................................... 49

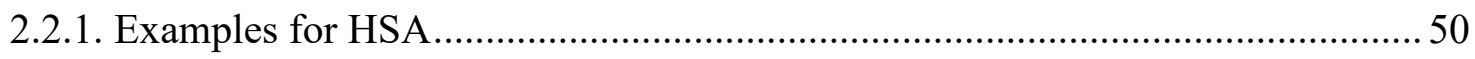

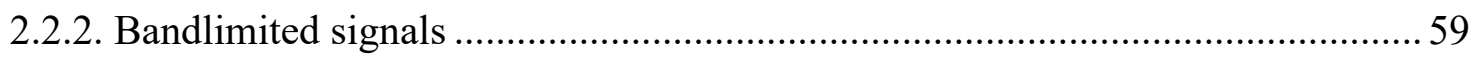

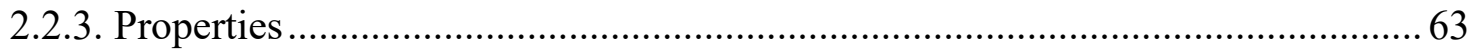

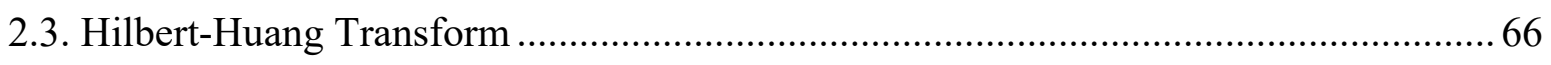

2.3.1. Empirical Mode Decomposition and Hilbert Spectrum Analysis ........................66

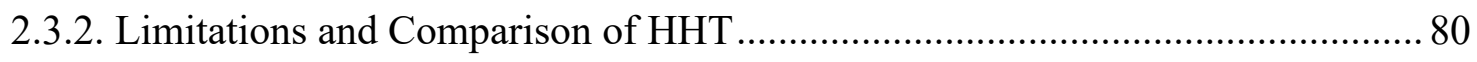

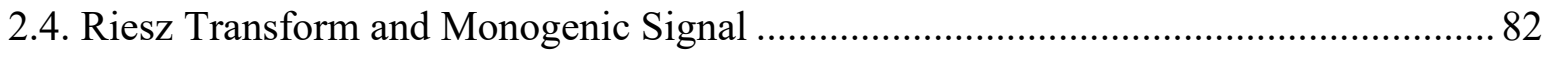

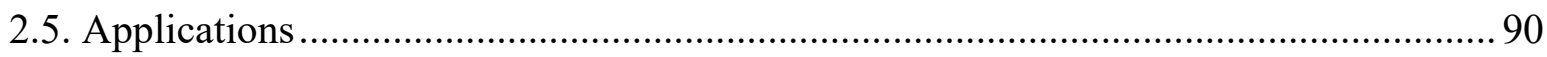

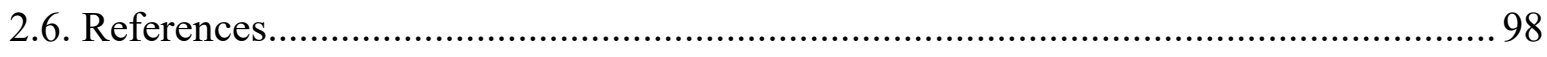

CHAPTER 3: Impact Damage Visualization in a Honeycomb Composite Panel through Laser

Inspection using Zero-lag Cross-correlation Imaging Condition ............................................ 104

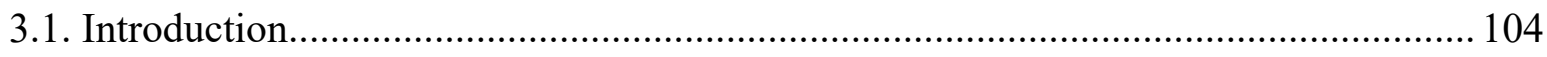

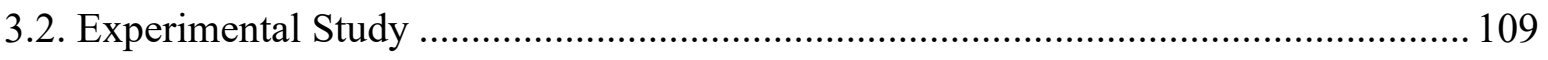

3.3. BVID Visualization using Zero-Lag Cross Correlation (ZLCC) Imaging Condition. 120

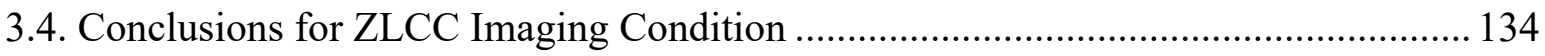

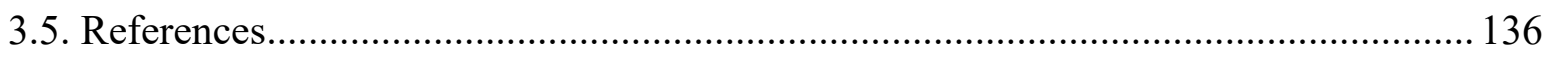

CHAPTER 4: Damage Imaging in a Stiffened Curved Composite Sandwich Panel with

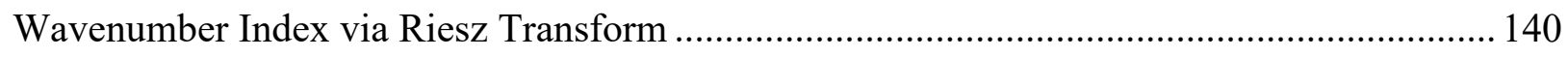

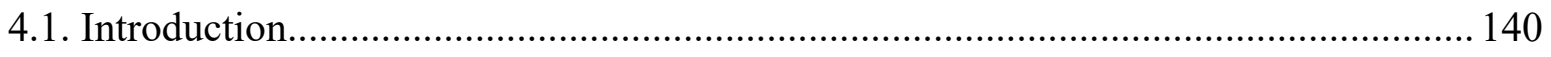

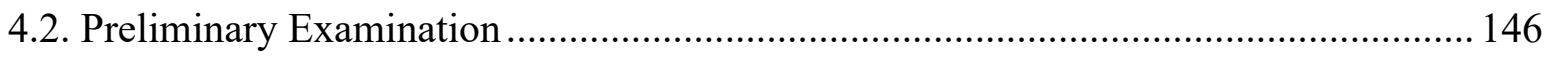

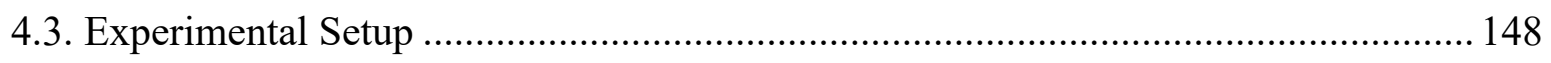

4.4. Monogenic Wavefield and Instantaneous Wave Vector ............................................. 154 
4.5. Phase Denoising and Damage Imaging via Wavenumber .....

4.6. Results and Comparison .................................................................................. 163

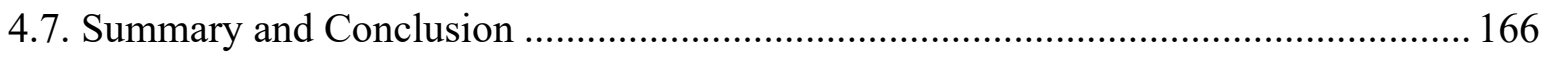

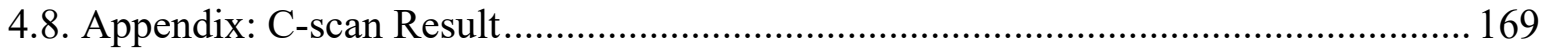

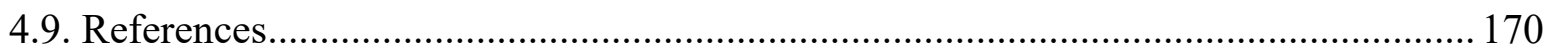

CHAPTER 5: Damage Visualization of Scattered Ultrasonic Wavefield via Integrated High-

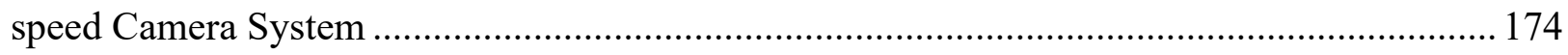

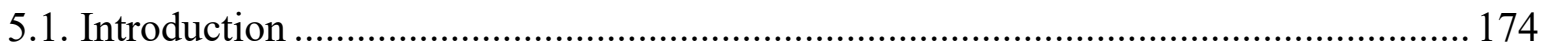

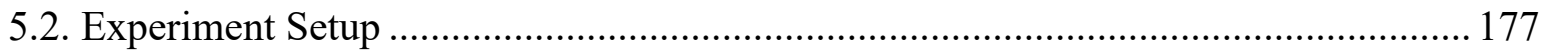

5.3. Logarithmic Value of Wavenumber Index (WI) .............................................. 179

5.4. Results and Comparison- Signal Frequency below the Nyquist Frequency ............... 181

5.5. Results and Comparison- Signal Frequency above the Nyquist Frequency .............. 185

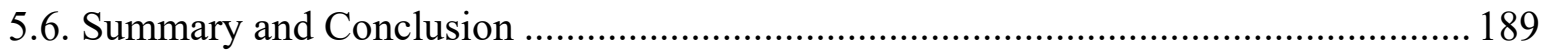

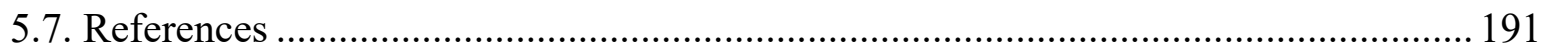

CHAPTER 6: Conclusion and Future Work.................................................................... 193

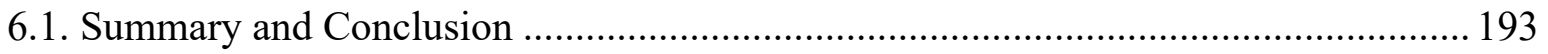

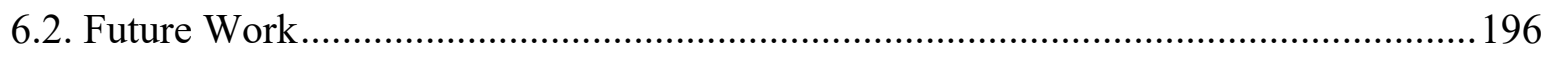




\section{LIST OF TABLES}

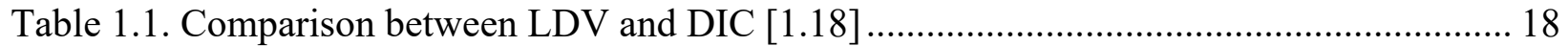

Table 2.1. Comparison between signal processing methods .............................................. 39

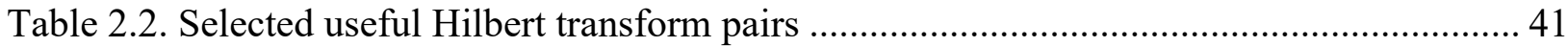

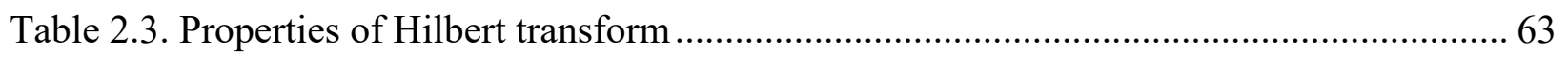

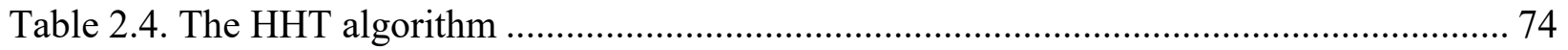

Table 3.1. Material Properties of TE-1 Grade 190 Type 35 Carbon/Epoxy Tape [3.25] .......... 131 


\section{LIST OF FIGURES}

Figure 1.1. The procedure to derive the local phase ………..................................................... 11

Figure 1.2. The illustration of feature tracking at a point ………............................................. 15

Figure 1.3. The illustration of feature tracking as a subset ..................................................... 15

Figure 2.1. Three-dimensional representation of analytic signal............................................... 35

Figure 2.2. (a) The real signal on the time-real plane; (b) the HT on the time-imaginary

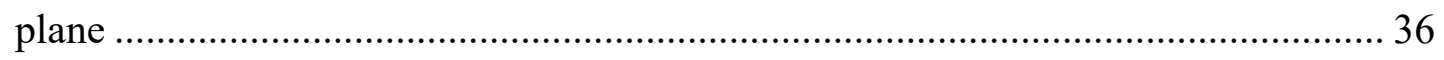

Figure 2.3. The analytic signal projected onto the complex plane ............................................... 37

Figure 2.4. The impulse response function $\mathrm{h}(\mathrm{t})$ in time domain ................................................... 44

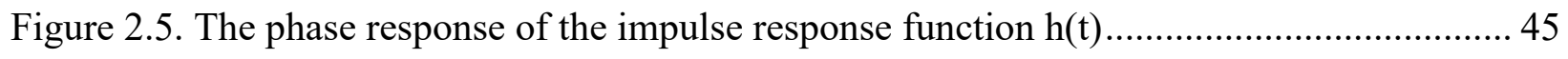

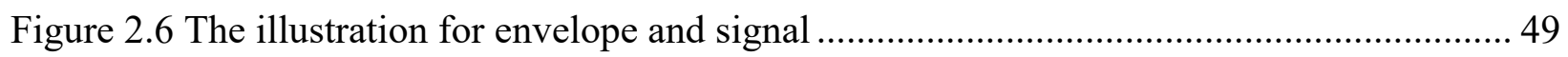

Figure 2.7. The HT, Envelope and the instantaneous phase ....................................................... 50

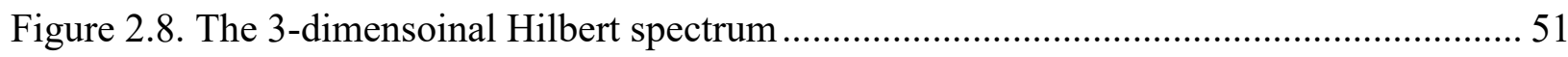

Figure 2.9. (a) The time-Frequency, (b) the Frequency-Magnitude, .......................................... 52

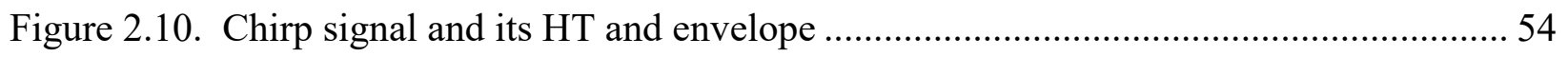

Figure 2.11. The instantaneous phase of Chirp signal from HT ................................................ 54

Figure 2.12. The instantaneous frequency of Chirp signal from HT ........................................ 55

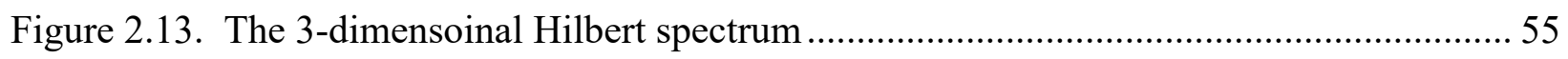

Figure 2.14. (a) The time-Frequency, (b) the Frequency-Magnitude, .......................................... 56

Figure 2.15. The Hilbert transform of a 5-cycle Hanning-modulated toneburst signal................. 57

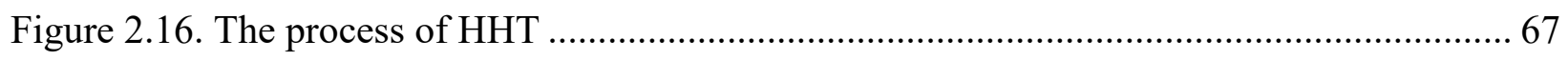

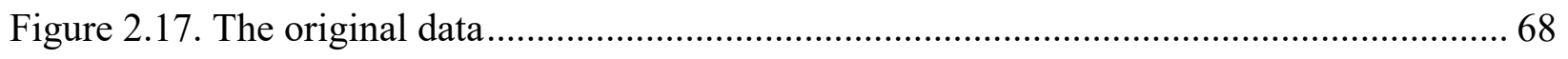

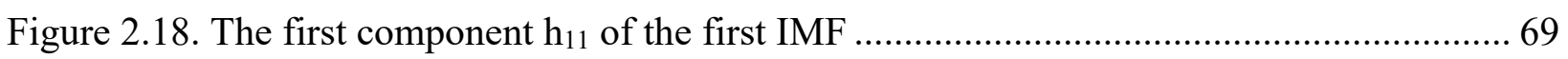




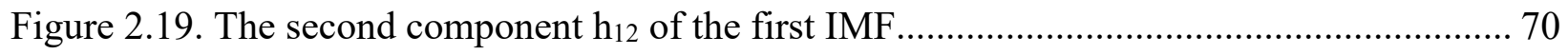

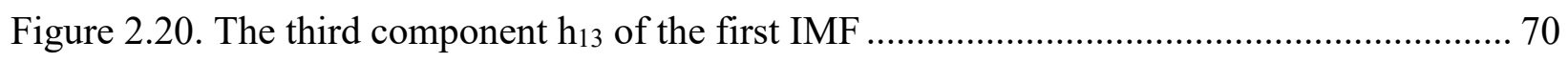

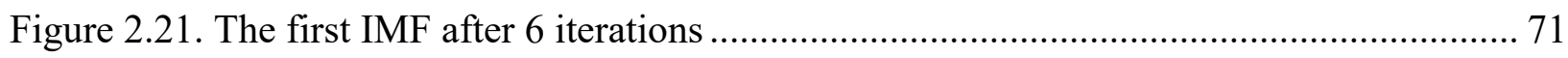

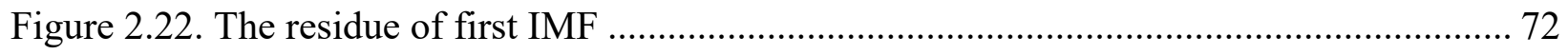

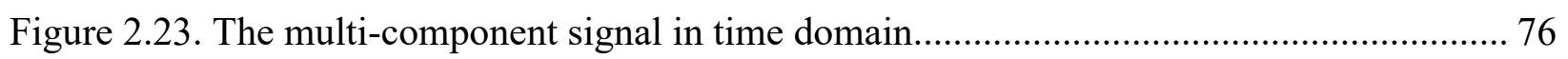

Figure 2.24. The FFT of the multi-component signal............................................................ 77

Figure 2.25. The Hilbert spectrum of the multi-component signal............................................. 77

Figure 2.26. The numerical (the blue curve) and theoretical (the red curve) IMFs, ..................... 78

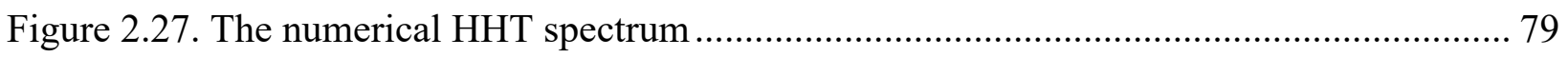

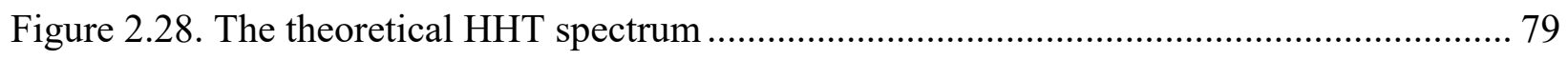

Figure 2.29. Illustrastion of Riesz transform operator in wavenumber domain along (a) xaxis as shown in Eq.(2.94), (b) y-axis as shown in Eq.(2.95)................................. 86

Figure 2.30. The illustration of wavefront, orientation and dominant phase direction as

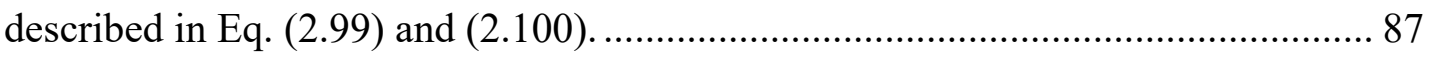

Figure 2.31. The wave filtering and ZLCC imaging method ................................................... 91

Figure 2.32. (a) Frequency-space domain, (b) Instantaneous wavenumber map, (c) Effective thickness map, and (d) Theoretical effective thickness map [2.33].. ........ 92

Figure 3.1. Major macro-damage failure modes of a honeycomb composite panel: core/face-sheet disbond, face-sheet delamination and core crush........................... 105

Figure 3.2. A schematic of the NDI system for imaging barely visible impact damage in honeycomb composite panel. ………………………….................................. 110

Figure 3.3. The setup of fully noncontact laser scanning system .............................................. 111 
Figure 3.4. The geometry of the composite sandwich panel; (a) perspective view; (b) side view (units: $\mathrm{mm}$ )

Figure 3.5. Equipment setup for ultrasonic scan of the composite panel: (a) C-scan system, (b) scanning head near the surface of the panel.

Figure 3.6. Ultrasonic C-scan images of (a) the dent area, (b) the cross-section view and (c) the dent location and size on the composite panel.

Figure 3.7. (a) Ultrasonic C-scan images of the delamination area, and (b) the delamination location and size on the composite panel.

Figure 3.8. X-ray CT scan of the impacted honeycomb composite panel: (a) top view from the scanned surface, indicating two elliptical delamination regions $17 \mathrm{~mm} \times$ $10 \mathrm{~mm}$ and $6 \mathrm{~mm} \times 4 \mathrm{~mm}$, and side views from three cross-sections (b) AA, (c) BB, and (d) $\mathrm{CC}$, revealing the depth and shape of the delaminations.

Figure 3.9. Excitation signal in the honeycomb composite panel generated by the pulse laser was measured by a nearby LDV.

Figure 3.10. Wavefield in the honeycomb composite panel at $\mathrm{t}=265 \mu \mathrm{s}$ : (a) prior to impact;

(b) after impact. 120

Figure 3.11. Wavenumber map at each frequency 123

Figure 3.12. Quadrant filtered wavenumber map 124

Figure 3.13. Filtered reflected wavefield at $\mathrm{t}=265$

Figure 3.14. Damage image using a simple RMS of out-of-plane velocity response

Figure 3.15. The decomposed damage imaged using ZLCC imaging condition at each frequency 128 
Figure 3.16. The damage imaged using ZLCC imaging condition at (a) $25 \mathrm{kHz}$, (b) $55 \mathrm{kHz}$,

(c) $80 \mathrm{kHz}$.

Figure 3.17. The wavenumber map showing standing wave phenomenon at (a) $25 \mathrm{kHz}$, (b) $55 \mathrm{kHz},(\mathrm{c}) 80 \mathrm{kHz}$.

Figure 3.18. Local resonant result from FEA (a) First mode around 24 kHz; (b) Second mode around $55 \mathrm{kHz}$.

Figure 3.19. The damage image using ZLCC imaging condition summed over the frequency range, $0-50 \mathrm{kHz}$.

Figure 3.20. The damage image using ZLCC imaging condition summed over the frequency range, $80-95 \mathrm{kHz}$.

Figure 4.1.(a) The geometry of the stiffened curved composite foam panel (not-to-scale),

(b) The illustration of ball-drop impact test, (c) The illustration for the cross section of the impact damage region close to the stiffener (not-to-scale).

Figure 4.2. The ultrasonic C-scan experimental setup and the result for the impact damage.

Figure 4.3. A schematic of laser scanning system for imaging the barely visible impact damage.

Figure 4.4. The illustration of LDV locations, scanning area and the crosssection of the panel

Figure 4.5. Excitation signal in the honeycomb composite panel generated by the pulse laser was measured by a nearby LDV. 151

Figure 4.6. The wavenumber map of the wavefield excited from four locations shown in Figure 4.4. The arrow indicates the wave propagating directed toward the 
damage region. (a) \#1, rightward, (b) \#2, leftward, (c) \#3, downward and (d) \#4, upward.

Figure 4.7. The snapshot of the wavefield "video" for (a) \#1, rightward, (b) \#2, leftward, (c) \#3, downward and (d) \#4, upward.

Figure 4.8. Illustrastion of Riesz transform operator in wavenumber domain along (a) $\mathrm{x}$ axis as shown in Eq. (4.5), (b) y-axis as shown in Eq. (4.6).

Figure 4.9. The illustration of wavefront, orientation and dominant phase direction as described in Eq. (4.10) and (4.11).

Figure 4.10. ZLCC results for LDV location and wave propagting direction: (a) \#1, rightward, (b) \#2, leftward, (c) \#3, downward and (d) \#4, upward.

Figure 4.11. WI results for LDV location and wave propagting direction: (a) \#1, rightward, (b) \#2, leftward, (c) \#3, downward and (d) \#4, upward.

Figure 4.12. The C-scan result shows all the delaminations at different depths beneath the impact location.

Figure 5.1. (a) The schematic system layout of experimental setup, and (b) the illustration of specimen setup in the test. 178

Figure 5.2. Frequency spectrum for $14 \mathrm{kHz}$ excitation at an arbitrary point before filtering for (a) x-displacement, and (b) y-displacement; after filtering for (c) $\mathrm{x}$ displacement, and (d) y-displacement

Figure 5.3. The wavefield snapshot at $800 \mu$ s for (a) $u_{x}$, (b) $u_{y}$ from camera, and (c) $u_{z}$ from LDV. The red dotted rectangle indicates the simulated damage at the back of the plate. 183 
Figure 5.4. The damage image generated from wavefield in (a) SH mode from camera, (b) $\mathrm{S}_{0}$ mode from camera, and (c) $\mathrm{A}_{0}$ mode from LDV. The red dotted rectangle indicates the simulated damage at the back of the plate.

Figure 5.5. Frequency spectrum for $28 \mathrm{kHz}$ excitation at an arbitrary point before filtering for (a) x-displacement, and (b) y-displacement; after filtering for (c) $\mathrm{x}$ displacement, and (d) y-displacement 186

Figure 5.6. The damage image generated from reconstructed wavefield via camera in (a) SH mode, (b) $\mathrm{S}_{0}$ mode. The red dotted line indicates the simulated damage at the back of the plate.

Figure 5.7. The damage image generated from reconstructed wavefield via camera in (a) $\mathrm{SH}$ mode, (b) $\mathrm{S}_{0}$ mode. The red dotted line indicates the simulated damage at the back of the plate. 


\section{CHAPTER 1 Introduction}

\subsection{Research Motivation}

Visual inspection is the most traditional and intuitive non-destructive inspection (NDI) method with long history and remains the most economical as well as fastest way for early assessment of health conditions of aircrafts [1.1]. Currently, over 80 percent of the inspections on large aircraft are done visually. As the time goes, visual inspection has evolved and been improved with the assistant of advanced technologies. Camera has been introduced to record the inspecting process, capture the image of the object and keep track of the history, providing better reliability, confidence, and somewhat automation. Recently, with the developed computer vision technique, camera has replaced human being in the inspection during manufacture or production. However, for the aviation industry, the visual inspection still relies on the knowledge and experience of the inspection personnel, and requires subjective judgement, both of which tend to be labor-intensive, tedious and inconsistent.

Advanced composite materials have played a major role for weight reduction, and the application in aerospace industry has increased significantly due to the excellent mechanical properties. The growth of the usage of composite materials, nevertheless, leads to even more difficult inspection, maintenance and repair. Most of the damages/defects in metallic structures such as fatigue and corrosion cracking can be found with visual inspection; however, in composite structures, damage types are diverse, complicated as well as nearly invisible from the surface, and therefore difficult to be identified with naked eye. In addition, composite structures are vulnerable to impact load due to the brittle behavior of the matrix and to the relatively thin thickness which does not allow efficient energy dissipation of impact energy; consequently, the low-velocity impact can induce significant internal delamination with little surface indentations which are too small to be 
visualized. The nearly invisible impact damage has increased the difficulty of visual inspection of composite structures for aircrafts and result in more time consumption, higher cost and even riskier.

Although some NDI techniques such as ultrasound C-scan and X-ray CT have shown to be effective in detecting such minute damages, they suffer disadvantages of being extremely costly and time-consuming. In most cases, the equipment is non-portable and the disassembly of the parts is required due to the limitation of dimension, which makes these techniques not suitable for early estimation of structural condition. Piezoelectric transducers and optical fibers are compact and lightweight with good sensitivity that can be used to detect anomalies in the structure by attaching/embedding them on/in the structure; however, the deployment of sensors and the wiring can be an issue as the amount of sensors increases. The fully non-contact, efficient and flexible techniques are hence highly preferred for the inspection of composite structures.

There are three non-contact NDI techniques most commonly used for inspecting hidden impact damages in composite structures: (1) Q-switched Nd:YAG pulse laser (QL); (2) air-coupled transducer (ACT); and (3) laser Doppler vibrometer (LDV). QL is a non-contact excitation source for generating broadband ultrasonic signals via thermal-elastic expansion effect; ACT can be used as either the excitation source or the sensor; and LDV can provide remote sensing with very good resolution. But they also possess certain drawbacks. For QL, the high intensity laser beam would endanger human health for the remote scanning; ACT needs to be operated at the vicinity of its resonant frequency, and the energy loss is significant due to the high acoustic impedance; and even though LDV performs very well for con-contact sensing with both high temporal and spatial resolution, it is much more expensive than other existing methods. 
For now LDV can be considered the most reliable and comprehensively used remote sensing technique providing precise measurement, but the technique also reveals several additional pitfalls in inhibiting practical area scanning: (1) the point-by-point scanning over the damage region by the LDV system is time-consuming; (2) the signal or event recorded by LDV must be absolutely repeatable or stationary; and (3) repeated excitations for the sampling may deteriorate the structure further after the inspection. At present, none of these NDI techniques can provide non-contact remote robust sensing capability to the inspection for composite structures of aircrafts.

Remarkable advances in digital technology for the past decade have tremendously enhanced the capability of digital camera in industries. The rapid growths of the performance of both cameras and computers have shown excellent potentials in the applications of NDI for aerospace. Recently, a motion magnification (MM) technique was developed by Computer Science and Artificial Intelligence Laboratory - known as CSAIL- at MIT. The technique allows commercial digital cameras to monitor imperceptible motions in videos by artificially amplifying small displacements through tracking feature change within video pixel by pixel, making them detectable by some automated techniques or even naked eye. The MM has been applied to visualize/analyze the vibration behavior for modal analysis. The combination of digital camera and laser interferometry, known as electronic speckle pattern interferometry (ESPI)/Shearography, has been shown as an effective NDI tool of damage detection for composite structures in several industries. Moreover, the digital image correlation (DIC) technique, which provides full-field non-contact 3D measurement by tracking the movement of the speckles on the object, has taken place of traditional sensors to measure deformations, vibrations and strains. Due to continuing and rapid advances of both hardware and software in cameras and computational algorithms, digital cameras of next generation without doubt will become the major player in the field of SHM/NDI. Even though 
several successful applications of digital cameras in NDI have been shown recently, none of them can meet the requirement of both spatial and temporal resolution for the inspection in composite structures due to the limitation of current technology.

In this dissertation, the ultimate goal is to overcome the limitation of the current hardware by increasing the equivalent sampling frequency and resolution of the high-speed camera, so that the concept of using camera for damage detection and visualization can be proved. Even though there are several damage imaging condition that have been developed to visualize the hidden damage using guided wavefield, these imaging conditions possess certain disadvantages. Therefore a new technique is needed for improving the sensitivity and flexibility, and it can be further customized for the wavefield captured by the high-speed camera. Once the new camera is developed in the near future, the algorithm and technique developed in this dissertation can be accommodated to the latest technique to achieve the goal of real-time damage visualization using high-speed camera.

\subsection{Motion Magnification}

\subsubsection{Introduction}

Motion magnification is a concept to magnify the imperceptible motion for human eye. These subtle changes can include plenty of valuable information. e.g., chest movement of a baby, parents keeping track of babies with their respiration; eye ball motion, the motion determining people's mental status. The technique needs only a standard video as input file, by applying temporal and spatial filters, a video can be decomposed into pixels for each frame. The subtle motion can be extracted and magnified to reconstruct the video revealing hidden information. 
There are several different techniques can achieve the goal. Eulerian motion magnification method was developed mathematically based on first-order linear Taylor-series expansion in 2012 [1.2], which was fast and simple, but limited in scaling factor and sensitive to noise characteristic. To avoid the noise from being magnified, complex steerable pyramids phase-based motion magnification was then developed [1.3] for increasing scaling factor and signal-to-noise (SNR) behavior, but it was computationally costly due to the over-completeness caused by multiorientation. Therefore, Riesz-pyramid was brought up [1.4] to replace the complex steerable pyramids for the computational efficiency while keeping similar image quality.

The motion magnification method has been recently applied to some research relevant to structural health monitoring. However, it was mostly just used for simple beam-like one-dimensional problem, and the application for composite plate still has not been discussed yet.

\subsubsection{Eularian Motion Magnification}

Eulerian motion magnification method [1.2] on a video image is the technique mathematically based on first-order linear Taylor-expansion, which is fast and simple. The concept is simple but can effectively amplify the displacement signal in the interest temporal frequency band. The frames were decomposed into pixels by applying spatial filters and the changes were extracted by applying temporal filters. The displacement between frames was magnified with an amplifying factor and added back to the original frame. The concept can be illustrated by a simplified onedimensional example. 
After being decomposed, $I(x, 0)$ is the initial intensity of the pixel located at coordinate $x$, and $I(x, t)$ is the intensity at the instant $t$. Assuming that the image undergoes a translational motion, the intensity of the pixel was moved for $\delta(t)$. The intensity initially located at $x$, after a period of time $\mathrm{t}$, moves to the new location of $x+\delta(t)$. Then the intensity can be expressed with respect to the displacement function $\delta(t)$.

$$
I(x, 0)=f(x) \Rightarrow I(x, t)=f(x+\delta(t))
$$

By applying the first-order Taylor series expansion, the intensity at time $t$ can be expressed as

$$
I(x, t) \approx f(x)+\delta(t) \frac{\partial f(x)}{\partial x}
$$

In order to pick out the displacement signal, a spatial bandpass filter was applied to the intensity function and the signal was extracted as

$$
B(x, t)=\delta(t) \frac{\partial f(x)}{\partial x}
$$

Giving a magnifying factor $\alpha$, the bandpass signal $B(x, t)$ can be multiplied by $\alpha$ and added back to $I(x, t)$, the processed signal $\tilde{I}(x, t)$ can be written as

$$
\tilde{I}(x, t) \approx f(x)+(1+\alpha) \delta(t) \frac{\partial f(x)}{\partial x}
$$

The processed signal can be applied with first-order Taylor expansion again, the signal then can be expressed as

$$
\tilde{I}(x, t) \approx f(x+(1+\alpha) \delta(t))
$$


This shows that the processing magnifies motions the spatial displacement $\delta(t)$ of the local image $f(x)$ at time $t$, has been magnified to a magnitude of $(1+\alpha)$. After being processed, the intensity value initially at coordinate $x$ moves to $x+\delta(t)$ originally, but now moves to $x+(1+\alpha) \delta(t)$ instead.

$$
I(x, t)=f(x+\delta(t)) \Rightarrow \tilde{I}(x, t) \approx f(x+(1+\alpha) \delta(t))
$$

The limitation of Eulerian motion magnification arises from the linear approximation of the firstorder Taylor series expansion. Due to the linear approximation, the error cannot be neglected when the displacement becomes large. Even though the Eulerian motion magnification is a simple method with fast processing, it is limited to small magnification factor, especially for high spatial frequency case and it is highly sensitive to noises. The disadvantages of linear approximation limit the feasibility of this method. Therefore the phase-based technique was further developed to circumvent the problem and to increase the feasibility and image quality.

\subsubsection{Complex steerable pyramids phase-based motion magnification}

As mentioned previously, Eulerian motion magnification method is based on linear approximation of first-order Taylor series expansion, which leads to the sensitivity to noises and the limited magnification factor. The phase-based motion magnification technique was then developed for overcoming the disadvantages, which was based on Fourier series decomposition.

Similar to the Eulerian motion magnification, the goal of phase-based motion magnification method is to extract the displacement function from the intensity function and to create the displacement-magnified intensity function. 


$$
I(x, t)=f(x+\delta(t)) \Rightarrow \tilde{I}(x, t) \approx f(x+(1+\alpha) \delta(t))
$$

As described in the previous part, the intensity function initially located at $\mathrm{x}$ is defined as $I(x, 0)$, and the intensity at the instant time $t$ is also defined as $I(x, t)$. By applying Fourier serious decomposition, the two intensity function can be decomposed to a summation of a series of complex exponential functions.

$$
\begin{gathered}
I(x, 0)=f(x) \stackrel{F F T}{\Rightarrow} \sum_{\omega=-\infty}^{\infty} A_{\omega} e^{i \omega x} \\
I(x, t)=f(x+\delta(t)) \stackrel{F F T}{\Rightarrow} \sum_{\omega=-\infty}^{\infty} A_{k} e^{i \omega(x+\delta(t))}
\end{gathered}
$$

After Fourier series decomposition, each sub-band $S_{k}(x, t)$ corresponds to a single spatial frequency $k$, and the phase term $k[x+\delta(t)]$ contains the motion information.

$$
S_{k}(x, t)=A_{k} e^{i k(x+\delta(t))}
$$

By applying a temporal filter, the DC component $k x$ will be removed, and the residue is the bandpassed phase $B_{k}(x, t)$. The bandpassed phase can then be multiplied by the amplifying factor $\alpha$ for increasing the phase of sub-band $S_{k}(x, t)$.

$$
\hat{S}_{k}(x, t)=S_{k}(x, t) e^{i \alpha B_{k}}=A_{k} e^{i k(x+\delta(t))} e^{i \alpha k \delta(t)}=A_{k} e^{i k(x+(1+\alpha) \delta(t))}
$$

The spatial displacement $\delta(t)$ of the local image $f(x)$ at time $t$ has been magnified by $(1+\alpha)$. The reason why phase-based motion magnification method has less sensitivity to noises is that the motion is magnified by shifting the phase, and the magnitude will not change during the process. Assuming the sub-band signal with noise is 


$$
S_{k}(x, t)=A_{k} e^{i k(x+\delta(t))}+\sigma_{n} N_{k}(x, t)
$$

As the previously mentioned procedure, the motion can be magnified by following procedure.

$$
\hat{S}_{k}(x, t)=S_{k}(x, t) e^{i \alpha B_{k}}=A_{k} e^{i k(x+(1+\alpha) \delta(t))}+\sigma_{n} e^{i \alpha k \delta(t)} N_{k}(x, t)
$$

The noise in the signal is only phase-shifted instead of being magnified. Therefore the phase-based technique can effectively solve the noise problem of linear approximation method.

To get the local phase and local translation, a quadrature pair is derived to form a complex number for using the Fourier phase shift theory; therefore, Hilbert transform can be used as a phase shifter.

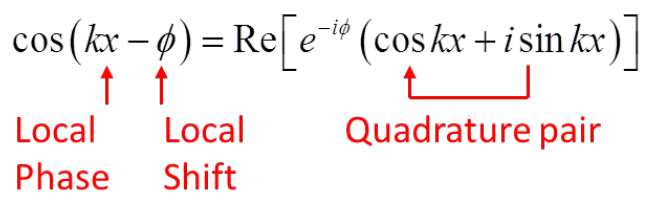

For any frequency $k$, the Hilbert transform of $f_{x}=\cos k x$ is $g_{x}=\sin k x$, implying Hilbert transform is a phase shifter, which gives any sinusoidal function $-90^{\circ}$ of phase shift. Considering all the motion as a cosine function, then we can get its quadrature pair, which is a sine function, after the Hilbert transform.

$$
H\{\cos (k x)\}=\cos \left(k x-\frac{\pi}{2}\right)=\sin (k x) \Rightarrow \cos (k x)+i \sin (k x)=e^{i k x}
$$

After the same phase shifting, the function will go forward for positive spatial frequency and go backward for negative spatial frequency.

$$
\begin{aligned}
& \text { for } \mathrm{k}>0 \Rightarrow e^{i k x} e^{-i \phi}=e^{i(k x-\phi)} \text {, which is shifted forward } \\
& \text { for } \mathrm{k}<0 \Rightarrow e^{-i k x} e^{-i \phi}=e^{-i(k x+\phi)} \text {, which is shifted backward }
\end{aligned}
$$


This phase-based motion magnification method introduced the concept to connect the magnitude and direction of the motion with the spatial phase derived from the image, and the maginified images obtained with the technique showed promising results to prove the feasibility and practicability. However, the phase is only defined in one dimension. There are at least two orientations needed to obtain the phase and orientation information from images, and in order to increase the accuracy of the reconstructed magnified images, typically more than eight orientations are used to ensure the precision, which leads to more computational cost. Therefore the Riesz transform was further introduced to the phase-based method to reduce the computational time while keeping the image quality.

\subsubsection{Riesz pyramid phase-based motion magnification}

Even though the complex steerable pyramid phase-based method resolved the problems of the sensitivity to noises and the limitation of magnifying factor for the Eulerian motion magnification based on liner approximation, it is much more computational costly for the multiple orientation of the steerable complex pyramid. The Riesz pyramid method is still a phase-based technique, but the Riesz pyramid is used for decomposition instead of original complex steerable pyramid.

The decomposition for multiple orientations in complex steerable pyramids method is timeconsuming due to implementation in frequency domain. Riesz Pyramids in this fast phase-based motion magnification is implemented in spatial domain. It can compute the dominant orientation and the local phase at every single point at the same time, so that it is more computing efficient. Riesz Transform is a generalization of Hilbert Transform; in two-dimension case, the Riesz Transform is a pair of filters with transfer functions as below. 


$$
-i \frac{k_{x}}{\|\vec{k}\|},-i \frac{k_{y}}{\|\vec{k}\|}
$$

By applying Riesz transform, the $90^{\circ}$ phase-shift in two orientations can be generated from the input sub-band. The dominant orientation can be further computed for each location from the two transforms. After getting the quadrature mate, the quadrature pair is formed and the local phase is known. Therefore the method can be computationally efficient without processing in frequency domain.

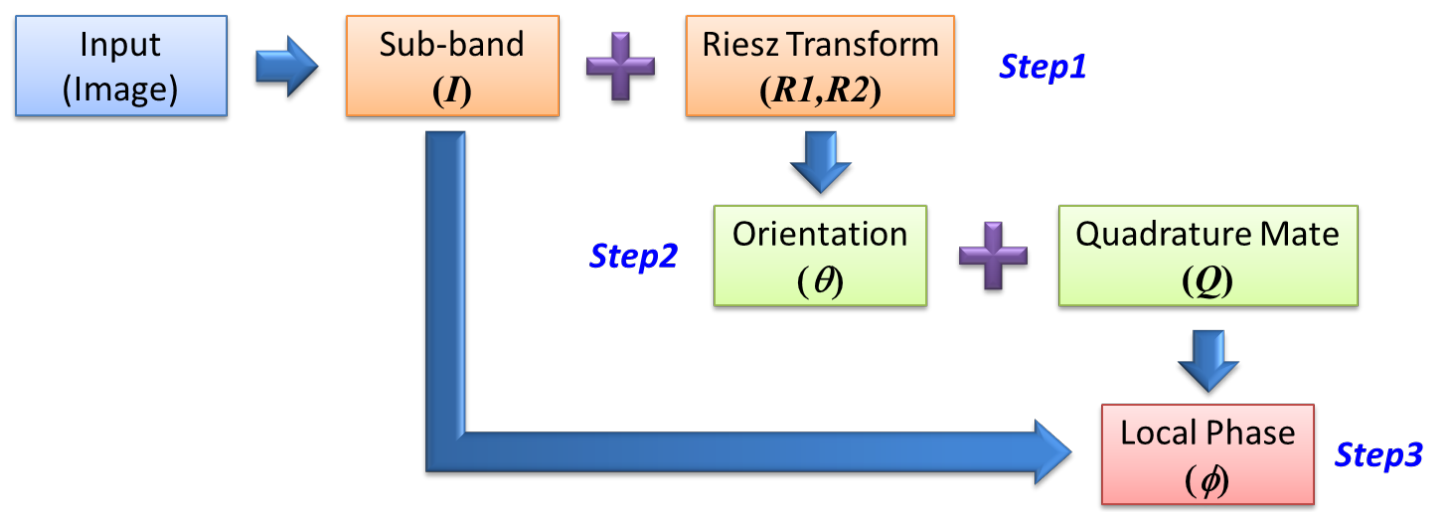

Figure 1.1. The procedure to derive the local phase

Assume the input sub-band function is $I=A \cos \phi$, where $A$ is amplitude and $\phi$ is local phase. The two Riesz transform results are $R_{1}=A \sin \phi \cos \theta$ and $R_{1}=A \sin \phi \sin \theta$, where $\theta$ is dominant orientation. The direction is invariant because of steerability, when arbitrary rotation angle $\theta_{0}$ equals to local dominant orientation $\theta$, the first component becomes the quadrature mate $Q=A \sin \phi$. The local dominant orientation and local phase for each point can thus be found for generating quadrature pair and applying phase-based motion magnification technique. 


$$
\left[\begin{array}{cc}
\cos \left(\theta_{0}\right) & \sin \left(\theta_{0}\right) \\
-\sin \left(\theta_{0}\right) & \cos \left(\theta_{0}\right)
\end{array}\right]\left[\begin{array}{l}
R_{1} \\
R_{2}
\end{array}\right]=\left[\begin{array}{c}
A \sin \phi \cos \left(\theta-\theta_{0}\right) \\
A \sin \phi \sin \left(\theta-\theta_{0}\right)
\end{array}\right]
$$

The Riesz pyramids method is still based on phase-based motion magnification, while using the Riesz pyramids in the spatial domain to replace the Complex Steerable Pyramids. After magnification, the noise characteristic and the magnification factor can almost keep the same as phase-based method while being much more computationally effective. This phase-based method with Riesz pyramid showed the effectiveness of deriving spatial phase and frequency, which gives a promising preliminary result for further analysis on wavefields using Riesz transform.

\subsection{Digital Image Correlation}

\subsubsection{Digital image correlation in NDI/SHM}

For damage detection, visualization and identification, traditionally signals are excited/measured on the surface for structural dynamic measurement by either contact or non-contact methods, e.g., a discrete piezo sensor array, the Nd-YAG pulse laser, and the laser Doppler vibrometer (LDV), and the signals are required to be linear, stationary and repeatable in order to reconstruct full-field signals from the point-by-point measurement, especially for guided wavefield reconstruction [1.6]. Guided wave-based techniques have been comprehensively employed to non-destructive inspection (NDI), structural health monitoring (SHM), and material characterization in plate-like structures [1.7]. However, current systems that can measure full wavefield propagation require significant set-up time, and collecting signal or scanning wavefield is time-consuming. 
Digital image correlation (DIC) is the solution that can circumvent these problems. The DIC technique is an optical technique employing feature tracking and image registration for extracting full-field displacement and strain fields from the change in images. The technique has been comprehensively applied for research and industrial testing purpose, and the recent advances in the camera technology and computer performance have been broadening the applicable field that requires high spatial and temporal resolution [1.8]. As the technology improves, the new highspeed cameras nowadays are showing good potentials to achieve full-field measurement for transient signals and to provide the most spatially continuous sampling compared to any other measurement methods with discrete sensing locations.

The high-speed camera records motion on the structure surface excited by a piezo actuator, and the in-plane/out-of-plane displacement can be further extracted by DIC using either single or multiple cameras. The DIC systems nowadays can achieve real-time observation of structural vibrational behavior [1.9] and the operational deflection shape (ODS) for SHM and NDI. However, even the most up-to-date model of high-speed DIC is still not fully capable of replacing LDV in guided wavefield measurement. Therefore, solutions for the difficulty on the measurement of the ultrasonic wave signal with extremely high frequency and the small magnitude are needed, due to the current limitation in the performance of high-speed cameras. In terms of solving the current hardware problem, the new algorithms are necessary to obtain the displacement with limited spatial resolution and temporal sampling frequency. Since the current DIC algorithm is not optimized for the ultrasonic measurement, a systematic integration is also required so that the signal-to-noise ratio can be further increased for the research shown in this dissertation. 


\subsubsection{Cross-correlation in displacement extraction}

Using cross-correlation for obtaining differences and correlations between datasets has been known for a long time, and the earliest application in dealing with digital images is known as in 1970s [1.10]. Some research and development of DIC in early phase was conducted by a group of researchers at the University of South Carolina (USC) in 1980s [1.11], and its DIC algorithm has been optimized and improved recently and has become a mature product in the industry and academy.

The most basic concept of feature tracking for displacement extraction is that given a point with unique signature in the undeformed image, then search and track for the same point in the deformed image which has the maximum similarity function using cross-correlation methods. However, in practice, a single point does not possess enough unique signature, hence considering the neighboring pixels are necessary for proper feature tracking. The collection of neighboring pixel values is called a "subset" in cross-correlation process, and the selected size for the subset affect the bias error and variance of the results [1.12]. Basically larger subset size leads to less error and variance with sacrificing the computational efficiency [1.13]. The principle requirement of feature tracking is that each signature needs to be unique and non-repetitive, and the pattern should be isotropic and high contrast for good signal-to-noise ratio; therefore the best feature is the random pattern. 


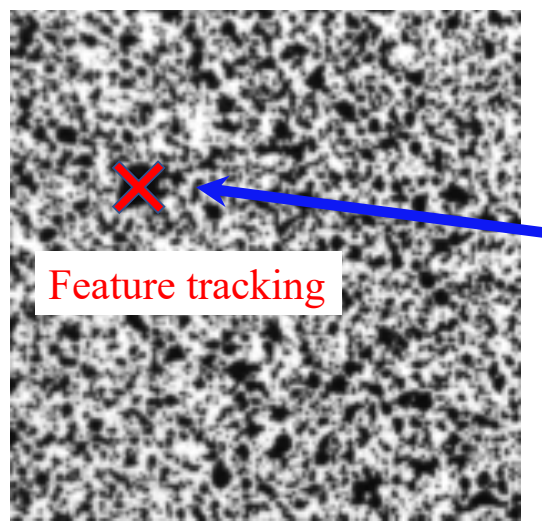

Time $t_{1}$

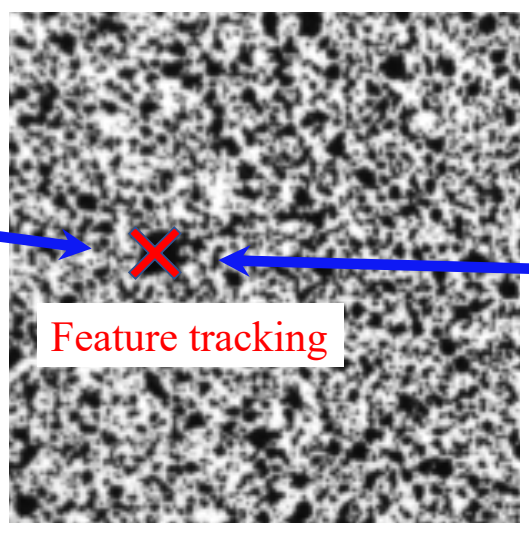

Time $t_{2}$

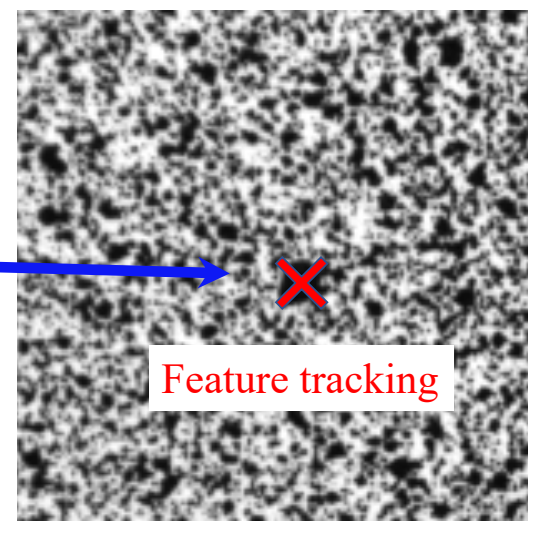

Time $t_{3}$

Figure 1.2. The illustration of feature tracking at a point

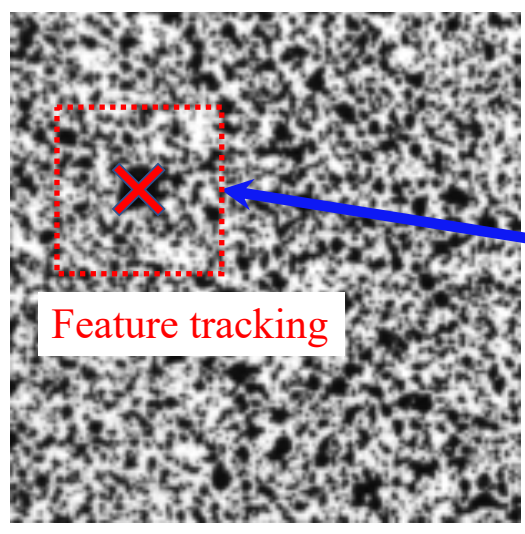

Time $t_{1}$

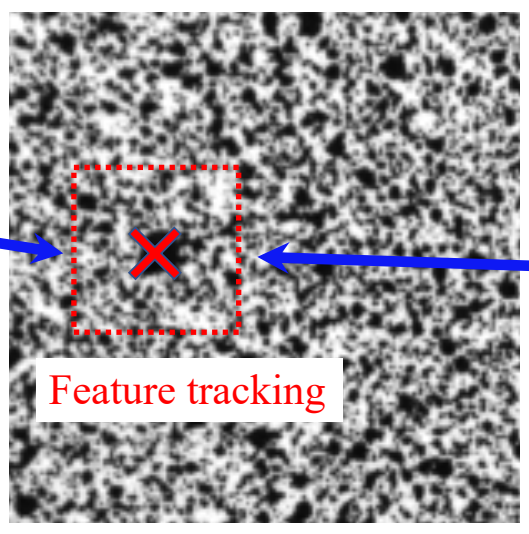

Time $t_{2}$

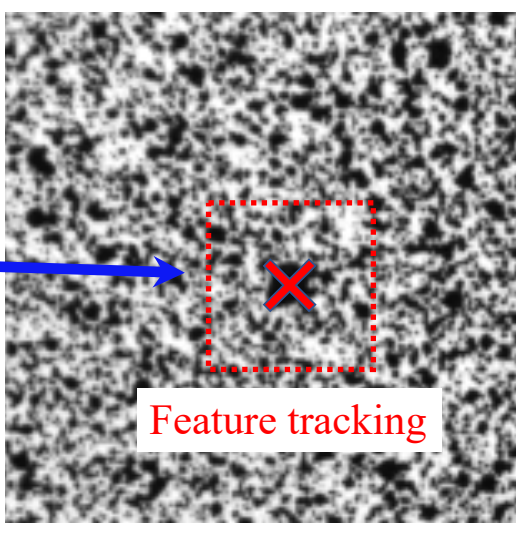

Time $t_{3}$

Figure 1.3. The illustration of feature tracking as a subset

For the full-field displacement extraction, the main task is to find where each subset moved by checking possible matches at several locations with a correlation function to obtain the maximum similarity between the deformed and undeformed images. The most classic correlation function is the sum of squared differences (SSD) of the pixel values, where the smaller value stands for better similarity between two subset. The basic SSD function $\mathrm{C}(x, y, u, v)$ is shown in Eq.(1.18), where $(x, y)$ is the pixel coordinate in the undeformed image, $(u, v)$ is the displacement of the subset in the 
deformed image, $n$ is the subset size, and $I$ and $I^{*}$ are the pixel value for the undeformed and deformed images. For perfect matching, the SSD value will be zero, which is the smallest value achievable. However, in real application, the value will never be zero because the images are always corrupted by noise. Interpolation technique will be needed in real application for images at non-integer pixel location.

$$
C(x, y, u, v)=\sum_{i, j=-n / 2}^{n / 2}\left(I(x+i, y+j)-I^{*}(x+u+i, y+v+j)\right)^{2}
$$

\subsubsection{Comparison of DIC and LDV}

Laser Doppler vibrometer (LDV) is one of the mature methods for collecting experimental measurements at discrete locations, and it has become well established in the vibrational, modal and wave analysis. Other optical measurement technique like digital image correlation (DIC) is relatively new in these applications. The main difference between LDV and DIC is that the LDV is only able to measure one point at each time, but DIC can measure multiple points within the measurement region simultaneously. Therefore, the measurement via LDV requires the excitation signal to be linear, stationary and repeatable in order to simulate that the reconstructed signal is measured at multiple points at the same time. The sequential measurement at each points for LDV requires longer times for obtaining the data, but the data itself is formatted and ready for further post-processing. In contrast, the DIC is time-efficient in measuring the data and it is able to deal with transient signal since it captures all the points at the same time, but the images obtained from camera require further correlation calculation in order to extract the full-field displacement. Therefore the computational time for DIC is humongous. 
Recent studies [1.14][1.15] have shown the possibility of using DIC in vibrational analysis, and DIC is found to be capable of generating full-field data from measurement. Other studies [1.16][1.17] have done comparison between LDV, DIC and traditional accelerometers for generating data in the experiment of modal analysis. Reu et al. [1.18] has recently done a detailed comparison of DIC and LDV with discussion for the pros and cons of each technique in practical vibration and modal measurement.

For using single devices, LDV can only measure out-of-plane displacement, and it requires multiple devices with further correlation process to be obtain the in-plane information. While for using single camera, DIC is only able to measure in-plane motion and requires multiple cameras and stereo correlation for out-of-plane information. Due to the nature of each technique, LDV is able to provide better out-of-plane displacement resolution and equivalent in-plane resolution to DIC. While LDV and DIC both has its advantages, DIC has definitely introduced a new and competitive approach for dynamic testing in various application fields. 
Table 1.1. Comparison between LDV and DIC [1.18]

\begin{tabular}{c|cc} 
Comparison Metric & LDV & DIC \\
\hline Cost & $\sim \$ 650 \mathrm{k}$ & $\sim \$ 350 \mathrm{k}$ \\
Setup Time & 2 hours & 2 hours \\
Acquisition Time & Hours & Seconds \\
Analysis Time & Seconds & Hours \\
Disp. Resolution & $\sim$ Picometers & $\sim$ Nanometers \\
Strain Resolution & N/A & 5 micron \\
Strain Calculation & Integrated- but researchy & Seamlessly integrated \\
Anti-aliasing & Included & Not possible at the moment \\
Data Volume & Small (Mbytes) & Large (Gbytes) \\
Software & includes only frequency data & but includes time history \\
& Designed for & In its infancy
\end{tabular}

\subsection{Guided Waves in Damage Detection for Composites}

Carbon fiber reinforced polymer (CFRP) composite materials have been widely used in lightweight structures in wind turbine, aerospace and automotive industries, etc. Sandwich composite is a typical type of application of carbon fiber, which is composed of multiple layers of CFRP as face-sheets and foam or honeycomb layer as the core, providing ultimate high strength/weight and stiffness/weight ratio of structures. However, the composite structures also increase the complexity of various failure modes and are rather difficult to identify damages, especially for sandwich structures. 
Unavoidable impacts occur to aircrafts during in-service operation, such as bird strikes or hail impacts. Furthermore, accidents may happen to regular inspection or maintenance leading to unexpected damages. For aluminum or steel structures, these impacts may cause dents or deformation on the surfaces with negligible reduction in strength or stiffness; however, for composite structures, impact load may cause delamination and debonding beneath surfaces, or even crush for the core material that can deteriorate the mechanical performance of materials significantly. These impact damages in composite materials are barely visible from the appearance, and they are difficult to be found during regular visual inspection, leading to potential safety issues and higher risk. Even though there are several robust non-destructive inspection (NDI) techniques that can not only detect but also visualize aforementioned barely-visible impact damage (BVID) in composites, such as ultrasonic scan or X-ray computed tomography (CT) scan, they are mostly not portable and time-consuming, and the techniques have spatial limitation that requires disassembly of the interrogated objectives [1.19].

Guided wave-based damage detection techniques have been studied for years to detect, locate and identify damages existing in the plate-like structures. Guided wave can propagate long distance without significant dissipation, and the longer it goes the more information it carries due to interactions with boundaries or any kind of discontinuity during its propagation. BVIDs in composite structures create discontinuity in mechanical properties which generates localized new wavenumber, wave reflection/refraction and sometimes generates local resonant vibrations, while guided waves propagates through the damage area; these phenomenon are helpful to distinguish the anomaly of the structure by analyzing the guided wave signal. Basically the more sensor/actuator used to collect/excite the wave signal, the more detailed damage information can be derived. One or few more sensors/actuator can be only capable of detecting the existence of 
damages by comparing the wave signals between pristine condition and damaged condition. Once the amount of sensor/actuator increases, the damages can be further located; array configuration is commonly used recently for damage localization than distributed sensor/actuator [1.20]. When the amount of the sampling point is large enough with ideal sampling spacing, the wave signal measured at each point can be reconstructed as a wavefield. The detailed shape and location of the damage can be visualized by analyzing the wavefield with a damage imaging technique.

Several damage imaging methods have been developed lately for damage visualization based on guided wavefield. Ruzzene et al. [1.21] first introduced root-mean-square (RMS) method which is the square root of the average sum of the wave signals squared, as shown in Eq.(1.19), to highlight the intensity difference at the damaged region for damage imaging. RMS can be considered as the most intuitive and simple method for damage imaging, it was further improved by Zak et al. [1.22] with weighted RMS and Saravanan et al. [1.23] with radially weighted and factored RMS for better damage imaging results.

$$
\operatorname{RMS}(x, y)=\sqrt{\frac{1}{N} \sum_{i=1}^{N} w_{T}^{2}\left(x, y, t_{i}\right)}
$$

where $w_{T}(x, y, t)$ is the total wave signal, $t_{i}$ is the initial time of the interested period, and $N$ is the amount of sampling point of the interested period. Sohn et al. [1.24], An et al. [1.25] and Park et al. [1.26] proposed a cumulative total wave energy (CTWE) technique with similar concept of RMS and a cumulative standing wave energy (CSWE) that shows more physical meaning to highlight local resonance behavior for damage visualization in composites. The standing wave energy is defined as the total wave signal squared minus the sum of propagating wave signals squared in four quadrants that were separated in frequency-wavenumber domain, as expressed in 
Eq.(1.20). Then the cumulative standing wave energy is the integration of the standing wave energy within the interested sampling period as shown in Eq.(1.21).

$$
\operatorname{SWE}(x, y, t)=w_{T}^{2}(x, y, t)-\sum_{p=1}^{4} w_{p}^{2}(x, y, t)
$$

where $w_{T}(x, y, t)$ is the total wave signal, and $w_{p}(x, y, t)$ is the propagating wave signal for $p=1,2,3,4$.

$$
\operatorname{CSWE}(x, y)=\int_{0}^{T} \operatorname{SWE}(x, y, t) d t
$$

where $T$ is the sampling period of interest.

Instead of using the localized high value in the amplitude of wavefield to highlight the damage region, Yuan and Harb [1.27][1.28] first introduced a zero-lag cross-correlation (ZLCC) damage imaging technique, where the incident and reflected waves separated in the frequencywavenumber domain were cross-correlated with zero time-lag to generate a cumulative damage image, as shown in Eq.(1.22). The incident wave and reflected waves are separated with a wavenumber filter along the interested direction, and ZLCC show the highest correlation value at the boundary of the damage where the reflected waves are generated. Girolamo et al. [1.29] further redefined the forward and backward propagating waves in improved two-dimensional wavenumber filtering process and employed ZLCC in frequency domain, as Eq.(1.23), to highlight the local resonant vibration within the damage region on a honeycomb composite panel. ZLCC employed in frequency domain shows better damage imaging result with less ambient noise and is capable of extracting the hidden resonant behaviors at higher local resonant frequencies. 


$$
I(x, y)=\int_{0}^{T} w_{i n c}(x, y, \tau) w_{r e f}(x, y, T-\tau) d \tau
$$

where $w_{i n c}$ and $w_{\text {ref }}$ are incident and reflected, respectively, wave signal in time-space domain separated with frequency-wavenumber filter.

$$
I(x, y)=\sum_{\omega=\omega_{i}}^{\omega_{f}} w_{i n c}(x, y, \omega) w_{r e f}^{*}(x, y, \omega)
$$

where $w_{\text {inc }}$ and $w_{\text {ref }}$ are incident and reflected, respectively, wave signal in frequency-space domain separated with frequency-wavenumber filter, and $\omega_{\mathrm{i}}, \omega_{\mathrm{f}}$ are lower and upper frequency respectively of the interested frequency range.

Furthermore, there are several damage imaging conditions taking advantage of localized wavenumber value to highlight the discontinuity in mechanical properties. Rogge and Lecky [1.30] introduced a wavenumber domain analysis via a mode separation technique followed by a spatialwindowed Fourier transform to obtain local wavenumber from the wavefield for characterization of impact damages in a composite plate. The developed wavenumber domain analysis provide good agreement with theoretical prediction of spatial window selection, and the damage imaging result shows the capability to characterize the depth of the delamination in composite laminate. Then the technique was further enhanced by Juarez and Lecky [1.31] to multi-frequency local wavenumber analysis along with a ply correlation technique to quantify the impact damage. With similar concept of using localized wavenumber to highlight the damage region, Mesnil et al. [1.32] proposed an instantaneous wavenumber estimation method for damage quantification, by employing one-dimensional spatial Hilbert transform on the wavefield for obtaining instantaneous wavenumber, as shown in Eq.(1.24), where $k(x, t)$ is the instantaneous wavenumber, $\hat{w}(x, y)$ is the 
Hilbert transformed signal of wave signal $w(x, y)$. The two-dimensional instantaneous wavenumber generalization was then defined as the square root of the sum of instantaneous wavenumber squared in two directions as shown in Eq.(1.25).

$$
\begin{gathered}
k(x, t)=\operatorname{imag}\left(\frac{\frac{\partial \hat{w}(x, t)}{\partial x}}{\hat{w}(x, t)}\right) \\
\operatorname{IW}(x, y, t)=\sqrt{k_{x}^{2}(x, y, t)+k_{y}^{2}(x, y, t)}
\end{gathered}
$$

Flynn et al. [1.33] introduced a damage imaging condition using local wavenumber estimation to image the structural features and defects. A wave mode separation technique was first applied onto the wavefield signal to isolate a narrow-band and single mode wave signal with its central frequency $f_{c}$ and central wavenumber $k_{\mathrm{c}}$, and local wavenumber $\hat{k}_{L O C}(x, y)$ were defined as the highest magnitude of the space-wavenumber representation for each point, as shown in Eq.(1.26). The space-wavenumber representation $S\left(x, y, k_{\mathrm{c}}\right)$ at given central wavenumber $k_{\mathrm{c}}$ is calculated by the sum of three-dimensional monogenic envelops of filtered wavefield signal at central wavenumber $k_{\mathrm{c}}$ within the interested sampling period as Eq.(1.27).

$$
\begin{gathered}
\hat{k}_{L O C}(x, y)=\underset{k}{\arg \max } S(x, y, k) \\
S\left(x, y, k_{c}\right)=\sum_{t=t_{0}}^{T}\left|w\left(x, y, t, k_{c}\right)\right|_{E}
\end{gathered}
$$

where $w\left(x, y, t, k_{\mathrm{c}}\right)$ is the wavefield signal with central wavenumber $k_{\mathrm{c}},|w|_{E}$ is the 3-D monogenic envelope, $t_{0}$ is initial sampling time, and $T$ is the total sampling period. 
In this dissertation, a new guided wave-based damage visualization method employing Riesz transform as an image processing technique was developed by the author and applied on a stiffened curved composite panel. The developed damage imaging technique, named wavenumber index (WI), took advantage of the abundant information contained in monogenic signals representation of the guided wavefield. The monogenic signal was derived by Riesz transform, the genuine twodimensional generalized Hilbert transform in space domain, to obtain instantaneous orientation, phase and amplitude of the constructed guided wavefield. The instantaneous wave energy was calculated from instantaneous amplitude, and the instantaneous wave vector was obtained by taking derivative with respect to two axes in space domain. The detailed description of WI method will be provide in Chapter 4.

\subsection{Objectives and Dissertation Outline}

The ultimate goal of this dissertation is to develop a robust fully noncontact vision-based NDI technique as a solution to remotely monitor the integrity and diagnose hidden damages in composite structures for aircrafts. The developed vision-based NDI system consists of (1) highspeed digital cameras with optimized illumination and surface treatment technique for the best sampling quality, (2) an automated scanning system for the rapid and effective diagnosis of large structures, and (3) the processing/controlling system to identify and visualize invisible damages via newly developed image processing technique.

Chapter 1 starts with the motivation of this whole research and following by the basic concept of motion magnification method which inspired the author to look into the vision-based technique. Then the general introduction of digital image correlation and guided wave based damage imaging 
condition are also provided in this chapter. The Hilbert transform and Riesz transform, which are two important keys in the technique of motion magnification, are introduced in details in chapter 2. Hilbert transform is a powerful time-frequency analysis tool that can be comparable with other methods such as short-time Fourier transform and Wavelet transform. The theory will be described and examples will also be given in the following chapter. The extension of Hilbert transform: Hilbert-Huang transform and Riesz transfom are both able to be applied for imaging processing will also be introduced in the next chapter.

In chapter 3, a zero-lag cross correlation damage imaging condition method that was applied on to a honeycomb composite panel for damage visualization is described in details. A fully non-contact laser-based nondestructive inspection (NDI) system was developed to detect and visualize damage in structures. The study focused on the size quantification and characterization of a barely visible impact damage (BVID) in a honeycomb composite panel. The hardware consisted of a Q-switched $\mathrm{Nd}$ :YAG pulse laser that probed the panel by generating broadband guided waves via thermoelastic expansion. The laser, in combination with a set of galvano-mirrors was used to raster scan over a two-dimensional surface covering the damaged region of an impacted quasi-isotropic [60/0/-60]s honeycomb composite panel. The out-of-plane velocities were measured at a fixed location normal to the surface by a laser Doppler vibrometer (LDV). An ultrasonic full wavefield assembled from the three-dimensional space-time data matrix in the interrogated area was first acquired and then processed for imaging the impacted damage area. A wavenumber filtering technique in terms of wave vectors was applied to distinguish the forward and backward wavefields in the wavenumber-frequency domain. A zero-lag cross correlation (ZLCC) imaging condition was then employed in the space-frequency domain for damage imaging. The ZLCC imaging condition consists of cross correlating the incident and reflected wavefields in the entire 
scanned region. The condition not only imaged the damage boundary between incident and reflected waves outside the damage region but also, for longer time windows, enabled to capture the momentary standing waves formed within the damaged region. The ZLCC imaging condition imaged two delaminated region: a main delamination, which was a skewed elliptic with major and minor axis lengths roughly $17 \mathrm{~mm}$ and $10 \mathrm{~mm}$ respectively, and a secondary delamination region approximately $6 \mathrm{~mm}$ by $4 \mathrm{~mm}$, however, which could only be shown at higher frequency range around $80 \mathrm{kHz}$ to $95 \mathrm{kHz}$. To conclude, the ZLCC results were in very good agreement with ultrasonic C-scan and X-ray computed tomographic (X-ray CT) scan results. Since the imaging condition was performed in the space-frequency domain, the imaging from ZLCC could also reveal resonance modes which were shown in the main delaminated area by windowing a narrow frequency band sequentially.

In chapter 4, imaging a damage using the phase of wavefield "video" was developed and applied to a stiffened curved composite panel, a section of an aileron from a Boeing C-17 Globemaster III, for visualization of a barely visible impact damage (BVID). The ultrasonic guided waves are generated by the thermoelastic effect induced by a Nd:YAG Q-switched pulse laser and then are captured point-by-point by a laser Doppler vibrometer (LDV). The wavefield "video" is reconstructed by measuring out-of-plane velocity on the surface of the panel. Newly generated wavenumbers from the geometry/material discontinuities caused by either the stiffener or the impact damage can be detected by observing the change of wavenumber from the reconstructed wavefield "video". The instantaneous wavenumber (the magnitude of instantaneous wavevector) derived via Riesz transform (RT) and its difference can be shown and highlighted using a proposed imaging condition, named as wavenumber index (WI). The WI sums the wavenumber values followed by a wave energy threshold filter which is performed in the time domain, which is in 
contrast to other imaging conditions implemented in the frequency-wavenumber domain by the use of complex wavenumber filtering and wave mode decomposition. Since WI is the phase-based imaging technique instead of conventional intensity-based technique for the wavefield "video", it is a robust technique with good computational efficiency that can cope with impact damages located in the vicinity of geometry/material discontinuities, yielding consistent damage image resolution with high sensitivity for wave propagation from all directions of the damage. The BVID of the composite structure becomes therefore "visible" with the proposed imaging technique. 


\subsection{References}

[1.1] Visual inspection for aircraft, Advisory Circular 43-204, Federal Aircraft Administration, 1997.

[1.2] H. Y. Wu, M. Rubinstein, E. Shih, J. Guttag, F. Durand, and W. Freedom, "Eulerian Video Magnification for Revealing Subtle Changes in the World,” 2012.

[1.3] N. Wadhwa, M. Rubinstein, F. Durand, and W. T. Freeman, "Phase-based Video Motion Processing," ACM Transactions on Graphics, vol.32, no.4, pp.80:1-80:10, 2013.

[1.4] N. Wadhwa, M. Rubinstein, F. Durand, and W. T. Freeman, ”Riesz Pyramid for Fast Phasebased Video Magnification," Computational Photography (ICCP), 2014 IEEE International Conference on. IEEE, pp. xx-xx, 2014.

[1.5] K. Kamble, N. Jagtap, R.A Patil and A. Bhurane, "A Review: Eulerian Video Motion Magnification," International Journal of Innovative Research in Computer and Communication Engineering, vol.3, no.3, 2015.

[1.6] D. Girolamo, H.Y Chang, and F.G. Yuan, "Impact Damage Visualization in a Honeycomb Composite Panel through Laser Inspection using Zero-lag Cross-correlation Imaging Condition," Ultrasonics, vol.81, pp.152-165, 2018.

[1.7] H.Y. Chang and F.G. Yuan, "Impact damage imaging in a curved composite panel with wavenumber index via Riesz transform," Proc. SPIE 10599, Nondestructive Characterization and Monitoring of Advanced Materials, Aerospace, Civil Infrastructure, and Transportation XII, p.105990L, 2018.

[1.8] P. Reu, "Introduction to digital image correlation: best practices and applications, "Experimental Techniques, vol.36, no.1, pp.3-4, 2012. 
[1.9] F. Trebuňa and M. Hagara, "Experimental modal analysis performed by high-speed digital image correlation system,” Measurement, vol.50, pp.78-85, 2014.

[1.10] P.E. Anuta, "Spatial registration of multispectral and multitemporal digital imagery using fast Fourier transform techniques," IEEE transactions on Geoscience Electronics, vol.8, no.4, pp.353-368, 1970.

[1.11] M.A. Sutton, C. Mingqi, W.H. Peters, Y.J. Chao and S.R. McNeill, "Application of an optimized digital correlation method to planar deformation analysis," Image and Vision Computing, vol.4, no.3, pp.143-150, 1986.

[1.12] H.W. Schreier and M.A. Sutton, "Systematic errors in digital image correlation due to undermatched subset shape functions," Experimental Mechanics, vol.42, no.3, pp.303-310, 2002.

[1.13] B. Pan, H. Xie, Z. Wang, K. Qian and Z. Wang, "Study on subset size selection in digital image correlation for speckle patterns," Optics express, vol.16, no.10, pp.7037-7048, 2008.

[1.14] M.N. Helfrick, C. Niezrecki, P. Avitabile, P. and T. Schmidt, “3D digital image correlation methods for full-field vibration measurement," Mechanical systems and signal processing, vol.25, no.3, pp.917-927, 2011.

[1.15] W. Wang, J.E. Mottershead, T. Siebert and A, Pipino, "Frequency response functions of shape features from full-field vibration measurements using digital image correlation," Mechanical systems and signal processing, vol.28, pp.333-347, 2012.

[1.16] C. Warren, C. Niezrecki, P. Avitabile and P. Pingle, "Comparison of FRF measurements and mode shapes determined using optically image based, laser, and accelerometer measurements," Mechanical Systems and Signal Processing, vol.25, no.6, pp.2191-2202, 2011. 
[1.17] D.A. Ehrhardt, S. Yang, T.J. Beberniss and M.S. Allen, "Mode shape comparison using continuous-scan laser Doppler vibrometry and high speed 3D digital image correlation," In Special Topics in Structural Dynamics, vol.6, pp.321-331, 2014.

[1.18] P.L Reu, D.P. Rohe, D.P. and L.D. Jacobs, "Comparison of DIC and LDV for practical vibration and modal measurements," Mechanical Systems and Signal Processing, vol.86, pp.2-16, 2017.

[1.19] F.G. Yuan, ed, Structural Health Monitoring (SHM) in Aerospace Structures, Woodhead Publishing, 2016

[1.20] Jiaze He and F.G Yuan, "Lamb-wave-based two-dimensional areal scan damage imaging using reverse-time migration with a normalized zero-lag cross-correlation imaging condition," Structural Health Monitoring, vol.16, no.4, pp.444-457, 2017.

[1.21] M. Ruzzene, S. M. Jeong, T. E. Michaels, J. E. Michaels, and B. Mi, "Simulation and Measurement of Ultrasonic Waves in Elastic Plates using Laser Vibrometry," AIP Conference Proceedings, Vol.760, no.1, pp.172-179, 2005.

[1.22] A. Zak, M. Radzienski, M. Krawczuk, and W. Ostachowicz, "Damage Detection Strategies based on Propagation of Guided Elastic Waves," Smart Materials and Structures, vol.21, p.035024, 2012.

[1.23] T.J. Saravanan, N. Gopalakrishnan, and N.P. Rao," Damage detection in structural element through propagating waves using radially weighted and factored RMS,” Measurement, vol.73, pp.520-538, 2015.

[1.24] H. Sohn, D. Dutta, J.Y. Yang, H.J. Park, M. DeSimio, S. Olson, and E. Swenson, "Delamination detection in composites through guided wave field image processing," Composites science and technology, vol.71, no.9, pp.1250-1256, 2011. 
[1.25] Y.K. An, B. Park, and H. Sohn, "Complete noncontact laser ultrasonic imaging for automated crack visualization in a plate," Smart Materials and Structures, vol.22, no.2, p.025022, 2013.

[1.26] B. Park, Y.K An, and H. Sohn, "Visualization of hidden delamination and debonding in composites through noncontact laser ultrasonic scanning," Composites science and technology, vol.100, pp.10-18, 2014.

[1.27] M.S. Harb and F.G. Yuan, "Impact damage imaging using non-contact ACT/LDV system," Structural Health Monitoring, vol.15, no.2, pp.2567-2574, 2015.

[1.28] M.S. Harb and F.G. Yuan, "Damage imaging using non-contact air-coupled transducer/laser Doppler vibrometer system," Structural Health Monitoring, vol.15, no.2, pp.193-203, 2016.

[1.29] D. Girolamo, H.Y Chang, and F.G. Yuan, "Impact Damage Visualization in a Honeycomb Composite Panel through Laser Inspection using Zero-lag Cross-correlation Imaging Condition,” Ultrasonics, vol.81, pp.152-165, 2018.

[1.30] M.D. Rogge and C.A. Leckey, "Characterization of impact damage in composite laminates using guided wavefield imaging and local wavenumber domain analysis," Ultrasonics, vol.53, no.7, pp.1217-1226, 2013.

[1.31] P.D. Juarez and C.A. Leckey, "Multi-frequency local wavenumber analysis and ply correlation of delamination damage," Ultrasonics, vol.62, pp.56-65, 2015

[1.32] O. Mesnil, C.A. Leckey and M. Ruzzene, "Instantaneous and local wavenumber estimations for damage quantification in composites," Structural Health Monitoring, vol.14, no.3, pp.193-204, 2015.

E.B. Flynn, S.Y. Chong, G.J Jarmer and J.R. Lee, "Structural imaging through local wavenumber estimation of guided waves," NDT \& E International, vol.59, pp.1-10, 2013. 


\section{CHAPTER 2 Image Processing via Hilbert and Reisz transform}

\subsection{Hilbert Transform}

\subsubsection{Introduction}

In Structural Health Monitoring (SHM), signal processing plays an essential role to physically interpret the collected data records from sensors, and further extract health status information of structures, and eventually predict the remaining useful life of the structure. Any time-varying signal will be best characterized by different signal attributed that change over time [2.1]. These attributes are usually described by amplitudes, instantaneous phases and frequencies. A brief history is introduced in this section for providing a general concept of signal processing tools in SHM. Back in 1743, Leonard Euler derived the formula

$$
e^{i z}=\cos z+i \sin z
$$

After 150 years later, Charles P. Steinmetz used this formula to introduce a complex exponential form for describing a linear wave equation under time-harmonic wave motion

$$
e^{i \omega t}=\cos \omega t+i \sin \omega t
$$

This complex exponential now is often used to describe the motion instead of using real-valued time-harmonic wave $\cos \omega t$ or $(\sin \omega t)$. Once the final solution is made in the complex domain, the real-valued solution can be taken by the real (or imaginary) part of the solution. Its logic is mainly from the Euler's formula

$$
e^{-i \omega t}=\cos \omega t-i \sin \omega t .
$$


A generalization can be made for a real-valued function $s(t)$ where a similar complex-valued function called analytic function is formed. By operating this complex-valued function, the resulting solution can be obtained by taking the real part of the complex-valued function. Using analytic function approach provides additional benefits in extracting such aforementioned parameters for describing signals.

In 1912, David Hilbert (1862-1943) introduced an integral transform, which is now known as Hilbert transform (HT). He showed that the function $\sin \omega t$ is the HT of $\cos \omega t$, and also provide the concept about the $\pm \pi / 2$ phase-shift operator as the basic property of HT. It was first introduced to signal processing in communication theory by Denis Gabor. Given a real-valued function $s(t)$, he defined a generalization of the well-known Euler formula in the form of the complex-valued function

$$
s_{+}(t)=s(t)+i \hat{s}(t)
$$

where $\hat{s}(t)$ is the Hilbert transform of $s(t)$.

In signal processing, when the independent variable is time, the associated complex-valued function is known as the analytic signal and the $\hat{s}(t)$ is the quadratic (or conjugate) function of $s(t)$. When dealing with general modulated signals, it is useful to use analytic signal for analyzing their features. Because signals in nature are real-valued, say through voltage, and it only represents the real part of the analytic signal, as referred to the analytic signal theory. The HT forms a basis of the definition of an analytic signal, which is the natural extension from real-valued signals to complex-valued signals, leading to one of the cornerstones in modern signal processing [2.2]. 
In contrast to other techniques where the variable is transformed from one domain to another, such as Fourier transform that transforms from the time domain to frequency domain, HT does in the same time domain. Since HT provides the complex representation of the analytic signal in the time domain, the signal processing can be expanded to the nonlinear system, rather than being limited in the linear, time-invariant systems as for Fourier transform. In addition, HT provides a direct examination to the initial signal of instantaneous attributes: amplitudes, phases, and frequencies, which yield to a good performance in time-frequency analysis. The time-frequency feature arises from the relation where the instantaneous frequency is the derivative of the phase. In the processing for vibrational signals, the real part of the transfer function can be derived from its imaginary part and vice versa. The use of transfer function enables nonlinear system identification and hysteretic damping characterization. For instance, for any vibrational or wave signals, it can be applied for deriving phases, frequencies or any other information needed. Since the HT of a signal is the quadrature pair of the original signal, the complex representation of the signal can be formed, which is called analytic (complex time) signal. The term "analytic" used is related to complex function of the complex variable. A simple example is provided below for better understanding to the concept of analytic signals. For a real signal $s(t)$ given as

$$
s(t)=3 e^{-\frac{2}{5} t} \sin 4 \pi t
$$

And its Hilbert transform $\hat{s}(t)$ is known as

$$
\hat{s}(t)=3 e^{-\frac{2}{5} t} \cos 4 \pi t
$$

The analytic signal $s+(t)$ can be formed from the real signal and its Hilbert transform as 


$$
s_{+}(t)=s(t)+i \hat{S}(t)=3 e^{-\frac{2}{5} t} e^{i\left(\frac{\pi}{2}-4 \pi t\right)}
$$

With an amplitude function $A(t)$ as

$$
A(t)=3 e^{-\frac{2}{5} t}
$$

The amplitude above can be considered as the envelope of both real signal and its HT. The original signal is the real part of the analytic signal, and the HT is the imaginary part of the analytic signal. Relationships between original, HT, analytic signal and its envelope can be clearly illustrated by a 3-dimensional representation of analytic signal given in the Figure 1.4. The spiral curve in magenta is the analytic signal in time domain, the real and the imaginary part is simply the projections onto time-real and time-imaginary plane. Similarly, the amplitude is also simply a projection on to the complex plane [2.3].

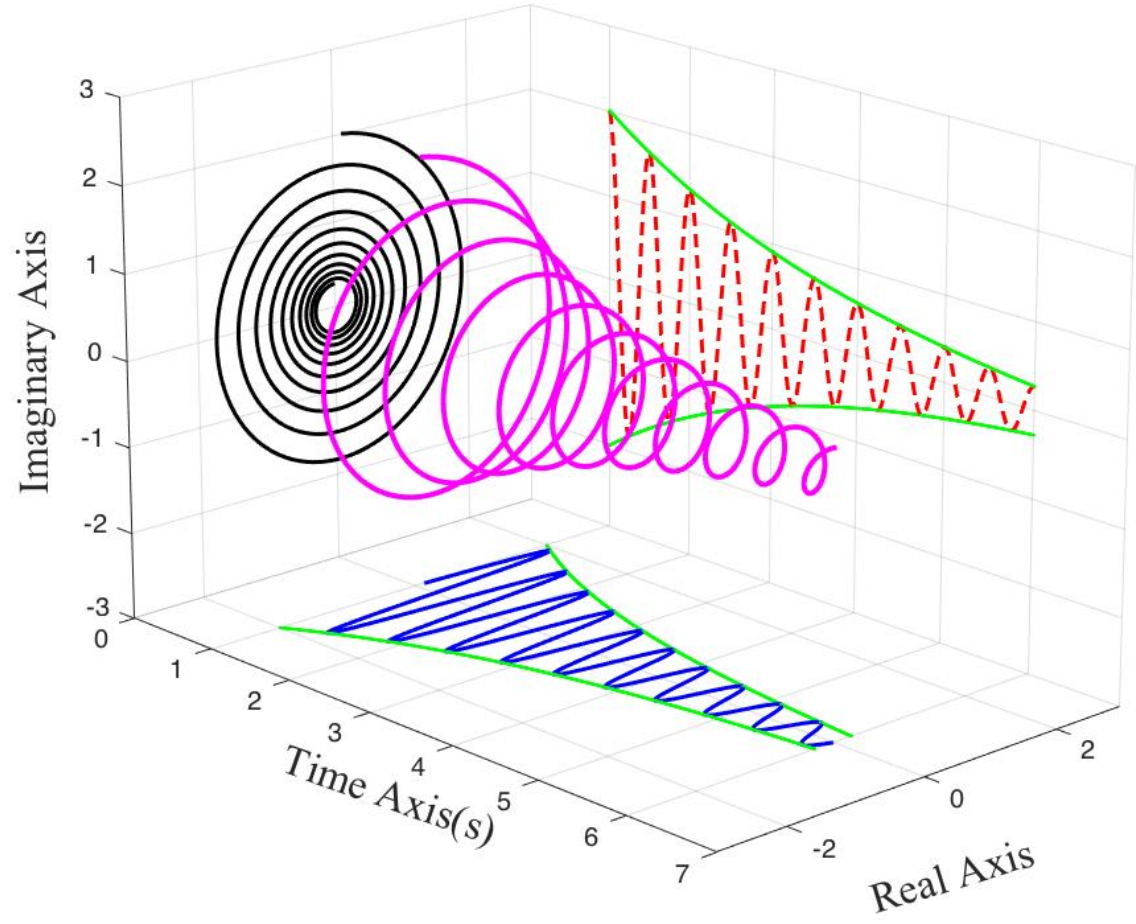

Figure 1.4. Three-dimensional representation of analytic signal. 
The blue solid curve on the Time-Real plane is the original real signal, and the red dash curve on the Time-Imaginary plane is the quadrature pair (HT). The amplitude is added on both real and imaginary part as the green envelope. The black curve on the complex plane (Real-Imaginary) is the projection of the whole analytic signal, and the $\phi(t)$ is the instantaneous phase, which is defined as the angle of the analytic signal on the complex plane.
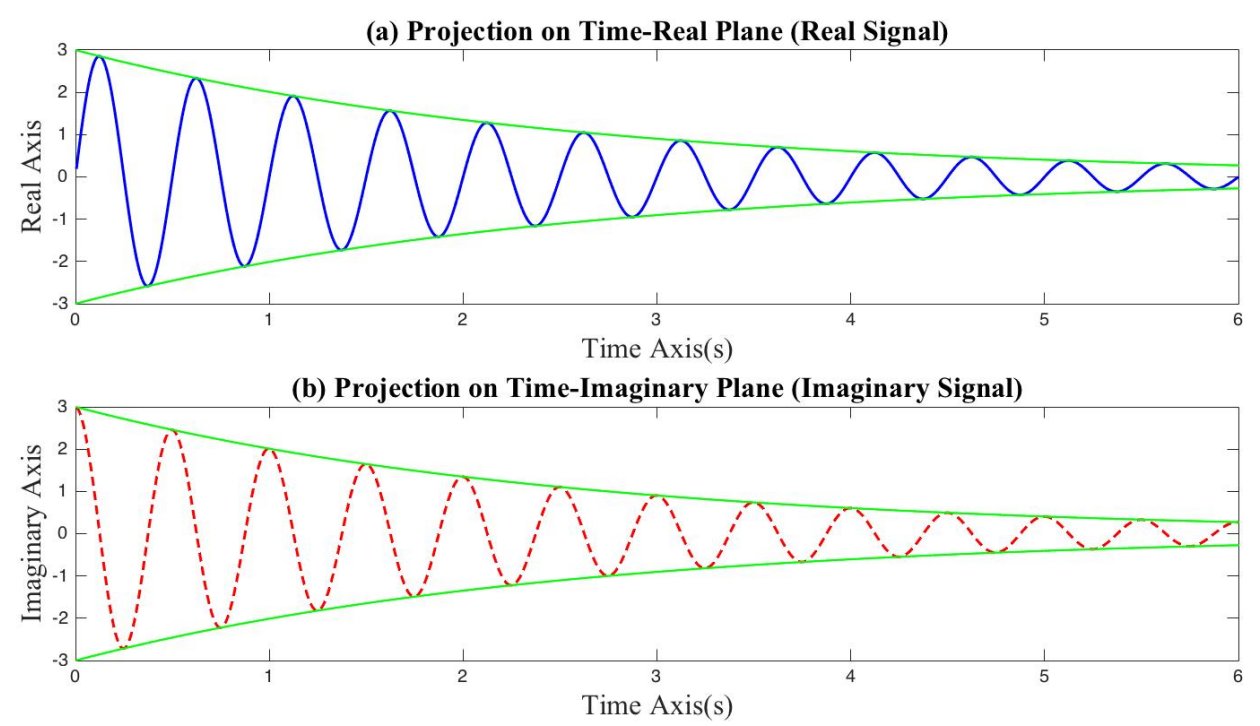

Figure 1.5. (a) The real signal on the time-real plane; (b) the HT on the time-imaginary plane 


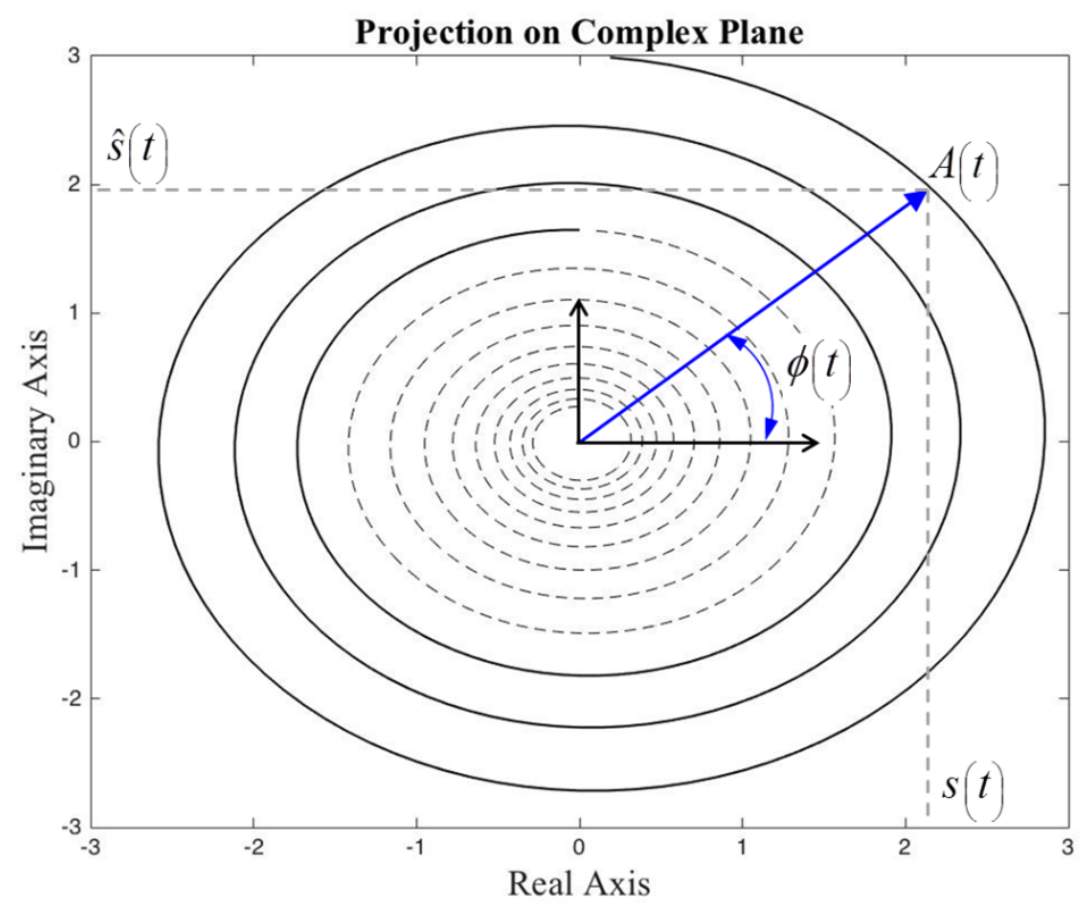

Figure 1.6. The analytic signal projected onto the complex plane

In 1998, Huang presented a new approach to analyze signals, called Hilbert-Huang transform (HHT). Its essential feature is the use of an adaptive time-frequency decomposition that does not impose a prior basis set on the data, and thus it is not limited by the time-frequency uncertainty, which Fourier or wavelet analysis encounters. This leads to a highly efficient tool for examining transient and non-linear effects. The HHT proceeds in two steps: First, the process of empirical mode decomposition (EMD) decomposed the signal into a set of mono-components, intrinsic mode function (IMF), thereby "sifting" or separating out different frequency scales of the data. The sifting is done adaptively, without a prior basic set imposed on the data, adaptively decomposes a signal into the simplest IMFs at the first step. Then, at the second stage, each decomposed IMF forms corresponding instantaneous amplitudes and frequencies, by utilizing HT on those obtained IMFs; energy-time-frequency distribution of the signal derived are designated as the HilbertHuang spectrum. Due to its instantaneous frequency property, HHT became more and more 
popular as a tool in transient signal processing. Wu and Huang further introduced a noise-assisted data analysis method, name ensemble empirical mode decomposition (EEMD) in 2009, for solving the mode-mixing problem with the original EMD method, by adding white noise evenly onto the signal during decomposition process [2.4]. With EEMD as the decomposition method, the HHT became more reliable and practical for dealing with signal in the real world.

Time-frequency analysis is widely employed in signal processing of SHM. The most well-known and widely used signal analysis tool, Fourier Transform, as referred to Lord Kelvin, is not only one of the most beautiful results of modern analysis, but may be said to furnish an indispensable instrument in the treatment of nearly every recondite question in modern physics. Even though Fourier Transform has diverse applicability, it is restricted to solving linear and stationary problem. Moreover, in Fourier representation, all the time-variant information is discarded, which means it cannot provide time-frequency analysis, leading to disadvantages in transient problems [2.5]. The other common time-frequency methods, such as Wigner-Ville distribution (WVD), short-time Fourier transform (STFT) and Wavelet transform (WT), etc., are also limited from extensive application or more accurate time-frequency information. For example, interference terms existing in the WVD induce some spurious components in time-frequency domain, which are physical meaningless. Moreover, the resolution in time-frequency domain of STFT and WVD is constant but could not be arbitrarily small since the signal is treated as stationary to admit Fourier transform. WT has been considered as an effective method for time-frequency analysis, but due to the limitation of Heisenberg-Gabor inequality, the WT cannot achieve fine resolutions in both time and frequency domain simultaneously. 
Consequently, it has demonstrated that the HHT has a higher resolution in time-frequency domain because of its instantaneous frequency concept, and better computational efficiency than either STFT or WT [2.6][2.7]. A brief comparison is listed in Table 1.2 for better understanding [2.8].

Table 1.2. Comparison between signal processing methods

\begin{tabular}{llll}
\hline Basis & Fourier & Wavelet & Hilbert \\
\hline Frequency & A priori & A priori & Adaptive \\
& $\begin{array}{l}\text { Convolution: } \\
\text { global uncertainty }\end{array}$ & $\begin{array}{l}\text { Convolution: } \\
\text { regional uncertainty }\end{array}$ & $\begin{array}{l}\text { Differentiation: } \\
\text { local certainty }\end{array}$ \\
\hline Presentation & Energy-frequency & Energy-time-frequency & Energy-time-frequency \\
\hline Nonlinear & No & No & Yes \\
\hline Non-stationary & No & yes & Yes \\
\hline $\begin{array}{l}\text { Feature } \\
\text { extraction }\end{array}$ & No & Discrete: no & Yes \\
\hline Theoretical base & Theory complete & Continuous: yes & \\
\hline
\end{tabular}

\subsubsection{Definition of Hilbert transform in time domain}

For a real-valued signal $s(t)$ of one-dimensional real-valued variable $t$, the Hilbert transform of $s(t)$, a kind of integral transform, is defined for all $t$ as $\hat{s}(t)$ by

$$
\hat{s}(t)=\mathcal{H}[s(t)]=-\frac{1}{\pi} \int_{-\infty}^{\infty} \frac{s(\tau)}{t-\tau} d \tau
$$

and the inverse Hilbert transform is 


$$
s(t)=\mathcal{H}^{-1}\left[(\hat{s}(t)]=\frac{1}{\pi} \int_{-\infty}^{\infty} \frac{\hat{s}(\tau)}{t-\tau} d \tau\right.
$$

where $s(t)$ and $\hat{s}(t)$ form a Hilbert transform pair.

This integral is improper in the sense that the integrand has a singularity $t=\tau$ at and the limits are infinite. This integral in Eq. (2.11) is evaluated as a Cauchy principal value of the integral, whenever this value exists. For simplicity, a symbol $P$ in front of the integral designating the principal value of the integral in most of the books is neglected herein. The Cauchy principal value is then defined as

$$
\hat{s}(t)=\mathcal{H}(\mathrm{s}(t))=\frac{1}{\pi} \lim _{\varepsilon \rightarrow 0^{+}}\left(\int_{t-1 / \varepsilon}^{t-\varepsilon} \frac{\mathrm{s}(\tau)}{t-\tau} d \tau+\int_{t+\varepsilon}^{t+1 / \varepsilon} \frac{\mathrm{s}(\tau)}{t-\tau} d \tau\right)
$$




\subsubsection{Hilbert transform pairs}

Some useful Hilbert transform pairs are selected and given in Table 1.3. [2.9][2.10][2.11]

Table 1.3. Selected useful Hilbert transform pairs

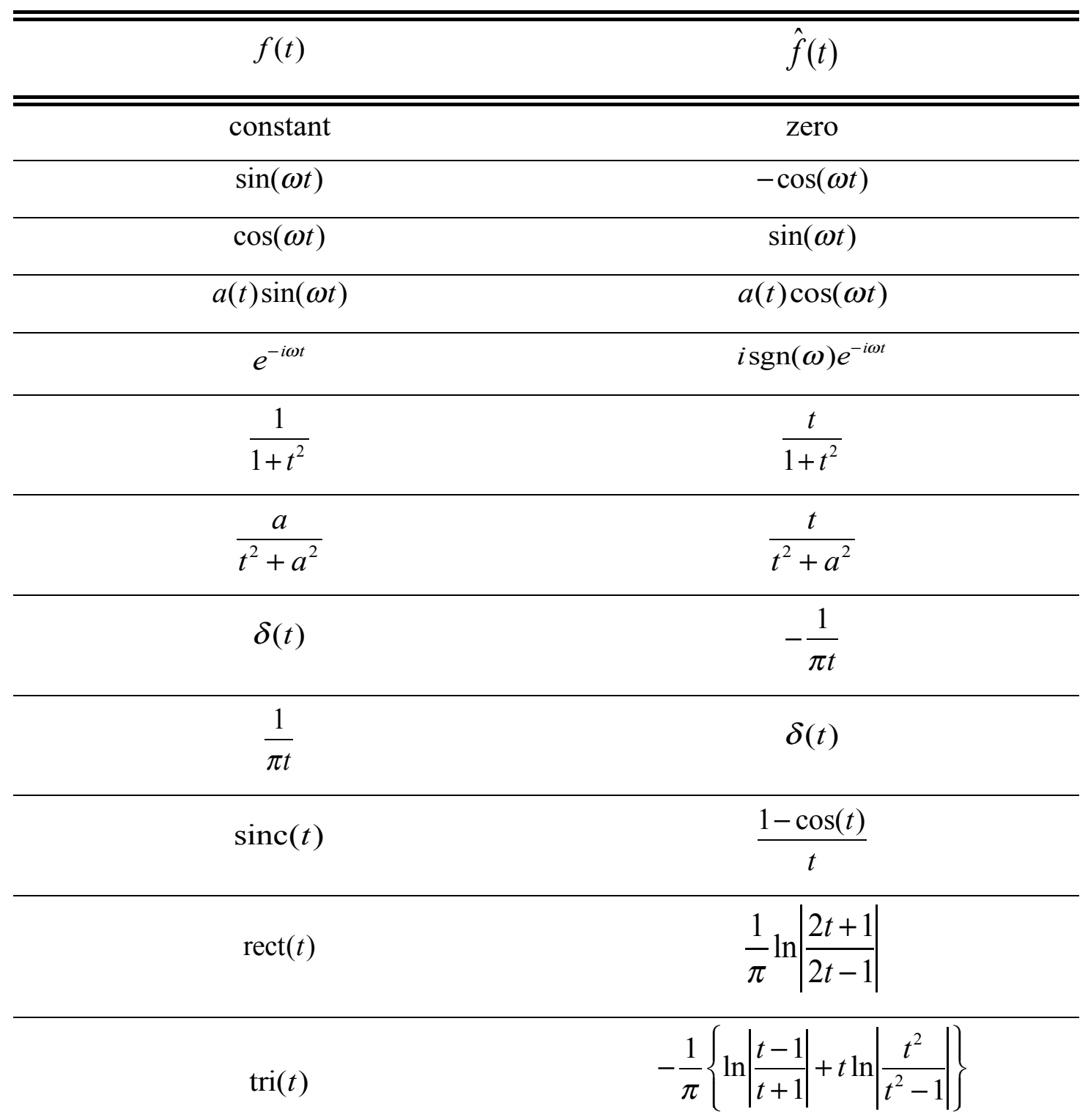


where $a$ is an arbitrary real-valued constant. For any constant, the Hilbert transform of the constant signal is zero. Thus HT eliminates any DC offsets. Two examples for calculating the Hilbert transform are provided. Given a real function $\cos \omega t$, the Hilbert transform can be calculated as

$$
\hat{f}(t)=-\frac{1}{\pi} \int_{-\infty}^{\infty} \frac{\cos \omega \tau}{t-\tau} d \tau
$$

Set $t-\tau \equiv \eta$, then

$$
\begin{aligned}
\hat{f}(t) & =-\frac{1}{\pi} \int_{-\infty}^{\infty} \frac{\cos \omega(t-\eta)}{\eta} d \eta \\
& =-\frac{1}{\pi} \int_{-\infty}^{\infty} \frac{\cos \omega t \cos \omega \eta+\sin \omega t \sin \omega \eta}{\eta} d \eta \\
& =-\frac{1}{\pi}\left[\cos \omega t \int_{-\infty}^{\infty} \frac{\cos \omega \eta}{\eta} d \eta+\sin \omega t \int_{-\infty}^{\infty} \frac{\sin \omega \eta}{\eta} d \eta\right] \\
& =-\sin \omega t
\end{aligned}
$$

where $\int_{-\infty}^{\infty} \frac{\cos \omega \eta}{\eta} d \eta=0, \int_{-\infty}^{\infty} \frac{\sin \omega \eta}{\eta} d \eta=\pi$. Obviously, $-\mathcal{H}^{2}[s(t)]=-\mathcal{H}\{\mathcal{H}[s(\mathrm{t})]\}=s(t)$ and hence $\mathcal{H}^{-1}=-\mathcal{H}$. Also, in order to calculate the Hilbert transform of a rectangular pulse function given by

$$
s(t)= \begin{cases}1, & \text { for }|x|<a \\ 0, & \text { for }|x|>a\end{cases}
$$

For $|t|<a$, the integrand has a singularity at $\tau=t$, and hence, 


$$
\begin{aligned}
\hat{s}(t) & =\frac{1}{\pi} \lim _{\varepsilon \rightarrow 0}\left[\int_{-a}^{t-\varepsilon} \frac{1}{\tau-t} d \tau++\int_{t+\varepsilon}^{a} \frac{1}{\tau-t} d \tau\right] \\
& =\frac{1}{\pi} \lim _{\varepsilon \rightarrow 0}\left\{[\ln |\tau-t|]_{-a}^{t-\varepsilon}+[\ln |\tau-t|]_{t+\varepsilon}^{a}\right\} \\
& =\frac{1}{\pi} \lim _{\varepsilon \rightarrow 0}\{\ln \varepsilon-\ln |a-t|+\ln |a+t|-\ln \varepsilon\} \\
& =\frac{1}{\pi} \ln \left|\frac{a-t}{a+t}\right|
\end{aligned}
$$

On the other hand, if $|t|>a$, the integrand has no singularity and hence

$$
\hat{s}(t)=\frac{1}{\pi} \int_{-a}^{a} \frac{d \tau}{\tau-t}=\frac{1}{\pi} \ln |\tau-t|_{-a}^{a}=\frac{1}{\pi} \ln \left|\frac{a-t}{a+t}\right|
$$

Finally,

$$
\hat{s}(t)=\frac{1}{\pi} \ln \left|\frac{a-t}{a+t}\right|
$$

\subsubsection{Definition of Hilbert transform in frequency domain}

The Hilbert transform pair can be conveniently written in the convolution notation in the following expression:

$$
\begin{gathered}
\hat{s}(t)=-\frac{1}{\pi t} \otimes s(t) \\
s(t)=\frac{1}{\pi t} \otimes \hat{s}(t)
\end{gathered}
$$

where $\otimes$ denotes convolution. Thus, the Hilbert transform of a signal can be explained as by 
passing it through a filter with the impulse response $h(t)=-\frac{1}{\pi t}$, thus clearly emphasizing the property of temporal locality of $s(t)$. Due to the convolution property, the Fourier transform of the $\hat{s}(t)$ can be expressed as the product of the Fourier transform of each function, which is given by

$$
\mathcal{F}[\hat{s}(t)]=\mathcal{F}[h(t)] \mathcal{F}[s(t)]
$$

Since applying Fourier transform to the impulse response function $h(t)$ gives

$$
\mathcal{F}[h(t)]=\mathcal{F}\left(\frac{-1}{\pi t}\right)=\int_{-\infty}^{\infty} \frac{-1}{\pi t} e^{i \omega t} d t=-i \operatorname{sgn} \omega
$$

, where

$$
\operatorname{sgn}(\omega)=\left\{\begin{array}{cc}
1 & , \text { for } \omega>0 \\
0 & , \text { for } \omega=0 \\
-1 & , \text { for } \omega<0
\end{array}\right.
$$

The impulse response in time domain is shown Figure 1.7. And the phase response of the impulse function is illustrated as Figure 1.8. It is clear that it shows the phase change $+90^{\circ}$ for positive frequencies, and the phase change $-90^{\circ}$ for negative frequencies.

\section{The Inpulse Function}

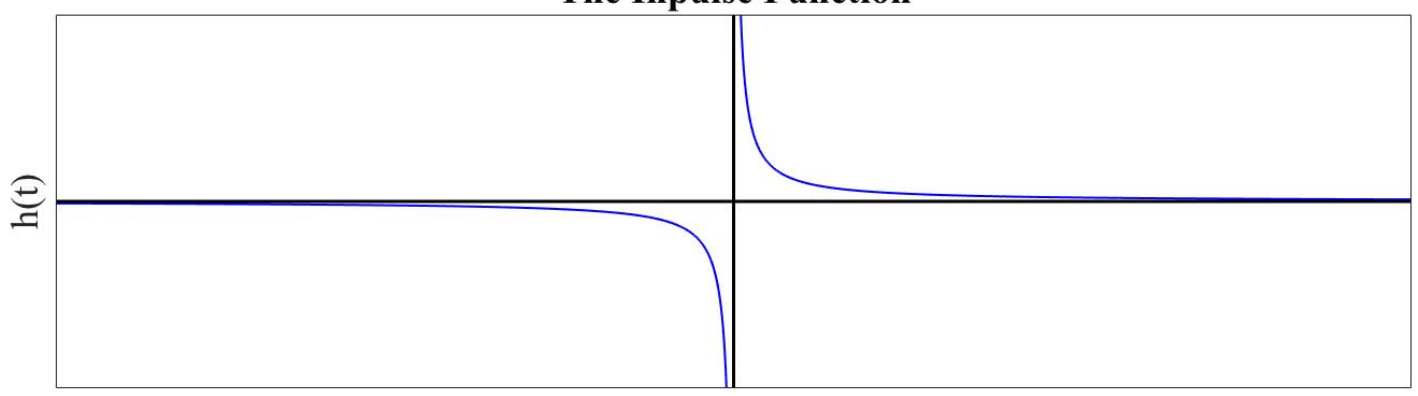

Time (t)

Figure 1.7. The impulse response function $h(t)$ in time domain 


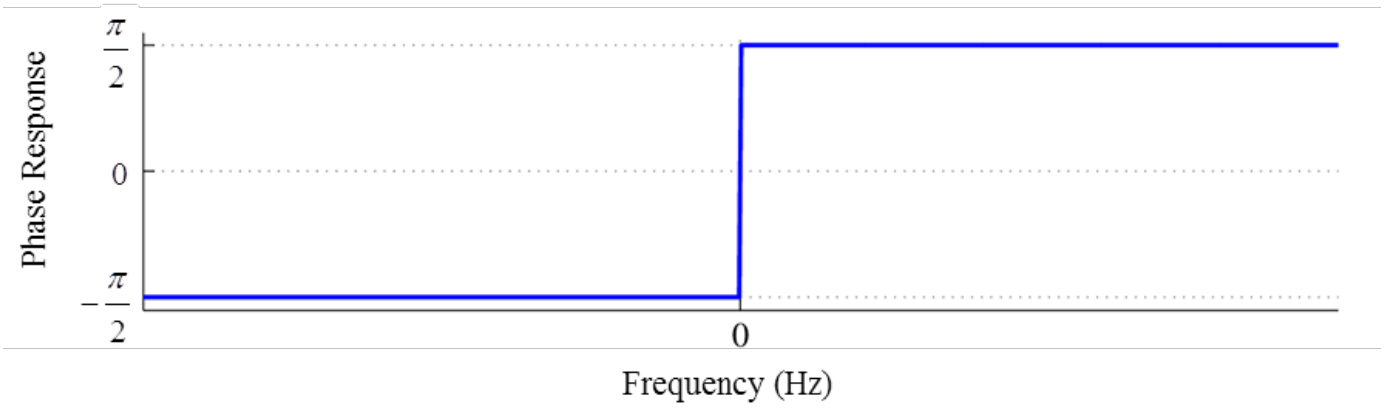

Figure 1.8. The phase response of the impulse response function $h(t)$

In the frequency domain, the Fourier transform of the Hilbert transform of $s(t)$ becomes

$$
\mathcal{F}[\hat{s}(t)]=-i \operatorname{sgn} \omega \mathcal{F}[s(t)]
$$

or

$$
\tilde{\hat{s}}(\omega)=-i(\operatorname{sgn} \omega) \tilde{s}(\omega)
$$

It means that in the frequency domain the HT is simply the multiplication of isgn $\omega$ to the function $\tilde{x}(\omega)$. The HT can be considered to be a filter which simply shifts phases of frequency components of its input by $i \operatorname{sgn} \omega$ and the amplitude does not change. Therefore in frequency domain, the result is then obtained by multiplying the spectrum of $x(t)$ by $i\left(+90^{\circ}\right)$ for positive frequencies and $-i\left(-90^{\circ}\right)$ for negative frequencies. The time domain result can be obtained by performing inverse Fourier transform. Therefore the HT of the original function $x(t)$ represents its harmonic conjugate. Obviously, $-\mathcal{H}^{2}[s(t)]=-\mathcal{H}\{\mathcal{H}[s(t)]\}=s(t)$ and hence $\mathcal{H}^{-1}=-\mathcal{H}$.

Now, giving a complex signal $s+(t)$, which is named analytic signal here, formed by a real signal $s(t)$ and its Hilbert transform $\hat{s}(t)$ 


$$
s_{+}(t)=s(t)+i \hat{s}(t)
$$

Taking the Fourier transform of the analytic signal gives

$$
\tilde{s}_{+}(\omega)=\tilde{s}(\omega)+i[-i \operatorname{sgn}(\omega) \tilde{s}(\omega)]=2 \operatorname{step}(\omega) \tilde{s}(\omega)
$$

where $\operatorname{step}(\cdot)$ is the Heaviside step function. Or it can also be expressed as

$$
\tilde{s}_{+}(\omega)=\left\{\begin{array}{cc}
2 \tilde{s}(\omega) & , \omega>0 \\
\tilde{s}(0) & , \omega=0 \\
0 & , \omega<0
\end{array}\right.
$$

Therefore the analytic signal $s_{+}(t)$ can be rewritten in terms of the inverse Fourier transform of its Fourier transform $s_{+}(t)[2.12]$ :

$$
\begin{aligned}
s_{+}(t) & =\frac{1}{2 \pi} \int_{-\infty}^{\infty} \tilde{s}_{+}(\omega) e^{-i \omega t} d \omega \\
& =\frac{1}{2 \pi} \int_{-\infty}^{\infty} 2 u(\omega) \tilde{s}(\omega) e^{-i \omega t} d \omega \\
& =\frac{1}{\pi} \int_{0}^{\infty} \tilde{s}(\omega) e^{-i \omega t} d \omega
\end{aligned}
$$

This equation concludes that $s_{+}(t)$ is an analytic (complex-valued) signal of $t$ for every $t$ in the upper half of the complex $t$ plane. In other words, the superfluous negative frequency components from Fourier transformed real-valued signal can be discarded using the complex-valued analytic signals, yet without loss of information.

Therefore, knowing that the theory of Hilbert transform is closely related to Fourier transform, Hilbert transform can be computed via Fourier transform in a few steps. First the real signal is 
Fourier transformed. Second, the negative frequencies are rejected. Finally, the inverse Fourier transform is performed. The resulting complex-valued signal where the real and imaginary parts form a Hilbert transform pair.

\subsubsection{Analytic signals and instantaneous parameters}

For an arbitrary signal $s(t)$, the analytic signal $s_{+}(t)$ is known as

$$
s_{+}(t)=s(t)+i \hat{s}(t)
$$

where the $\hat{s}(t)$ is the Hilbert Transform of the original signal $s(t)$. The analytic signal can be expressed in a polar form with amplitude and phase where the derivative of the phase can be identified as the instantaneous frequency.

$$
f(t) \equiv A(t) e^{i \phi(t)}
$$

The amplitude $A(t)=\sqrt{|g(t)|^{2}+|\hat{g}(t)|^{2}}$ of the analytic signal is the envelope (or amplitude) of the original signal. The instantaneous phase

$$
\phi(t)=\tan ^{-1}[\hat{g}(t) / g(t)]
$$

Note that phase angle jumps between $-\pi$ and $\pi$, because of the definition range of $\tan ^{-1}$ function. In this case, these phase jumps can be unwrapped into a monotone function by artificial changing of the phase value. Then the instantaneous frequency can be defined as

$$
\omega(t)=d \phi(t) / d t
$$


The time-variant amplitude (or energy, the amplitude squared) and frequency can be represented by a marginal spectrum defined by

$$
h(\omega)=\int_{0}^{T} H(\omega, t) d t
$$

Where $H(\omega, t)$ denotes the amplitude as a function of time and frequency and $[0, \mathrm{~T}]$ is the time domain within which the data is defined. The marginal spectrum represents the accumulated amplitude (energy) over the entire data span. Therefore, if there is a general modulated real signal given as

$$
f(t)=\left[A-M \cos \left(\omega_{m}+\phi\right)\right] \sin \left(\omega_{c}\right)
$$

where $\omega_{\mathrm{c}}$ is the center (or carrier) frequency and $\omega_{\mathrm{m}}$ is modulating frequency. The signal envelope can be found using the Hilbert Transform. For a special case where $M / A=1(100 \%$ modulation) and $\phi=0$, the modulated signal becomes

$$
f(t)=A\left[1-\cos \left(\omega_{m} t\right)\right] \sin \left(\omega_{c} t\right)
$$

The Hilbert Transform of the modulated signal is computed as

$$
\hat{f}(t)=-A\left[1-\cos \left(\omega_{m} t\right)\right] \cos \left(\omega_{c} t\right)
$$

The instantaneous amplitude $A(t)$, as aforementioned, is known as

$$
A(t)=\sqrt{|f(t)|^{2}+|\hat{f}(t)|^{2}}= \pm A\left[1-\cos \left(\omega_{m} t\right)\right]
$$

The instantaneous phase $\phi(t)$ in the time domain is then given by 


$$
\varphi(t)=\tan ^{-1} \frac{\hat{f}(t)}{f(t)}=\tan ^{-1}\left(-\cot \left(\omega_{c} t\right)\right)
$$

To conclude, Hilbert transform provide good applicability in the time-frequency analysis, especially for transient problem, due to its instantaneous properties

Assuming the analytic signal is $f(t)=[\cos (3 t)-2 \sin (4 t)] e^{i 50 t}$, the envelope signal is then $f_{e}(t)=\cos (3 t)-2 \sin (4 t)$, as the illustration in Figure 1.8. The envelope can be considered as the low-pass signal of the analytic signal. According to the Nyquist sampling theory, any signal must to be sampled at least two times the highest frequency. By separating the envelope and the carrier frequency, it is possible to sample the signal with lower sampling rate.

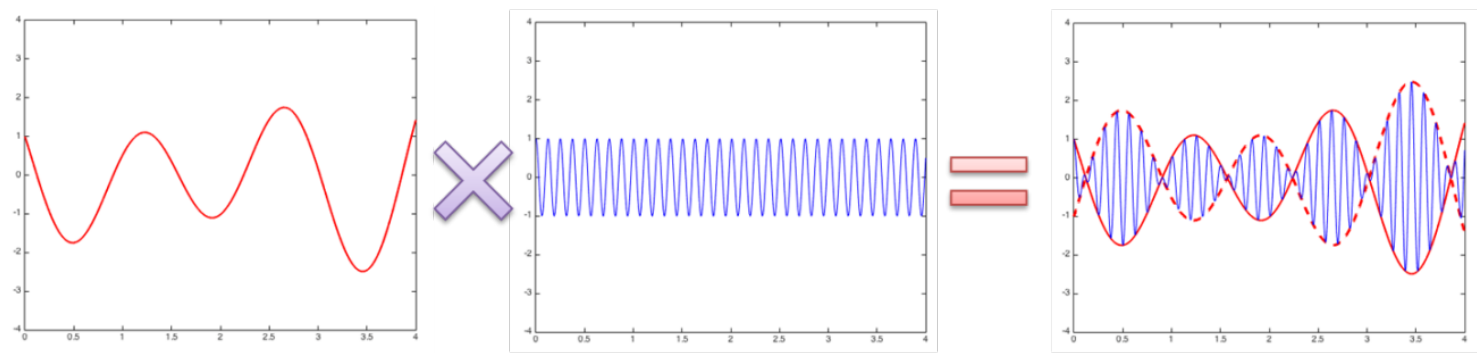

Figure 1.9 The illustration for envelope and signal

\subsection{Hilbert Spectral Analysis}

Since Hilbert transform (HT) provides abundant information such as instantaneous amplitude, phase and the instantaneous derived by taking derivative with respect to time, HT has been widely employed as a powerful time-frequency analysis tool. In this section, there are some example provided using the most common and frequently used signals in structural health monitoring. 


\subsubsection{Examples for HSA}

\section{$\underline{\text { Example: Sinusoidal Signal }}$}

Given a simple mono-component signal as

$$
s(t)=\sin 4 \pi t
$$

The Hilbert transform of sine function is exactly a cosine function with the same amplitude as aforementioned. In this case, since the instantaneous frequency derived is simply a constant, the slope of the instantaneous phase is constant. The Hilbert transform, the envelope and the phase can be illustrated along with the original signal as Figure 1.10.
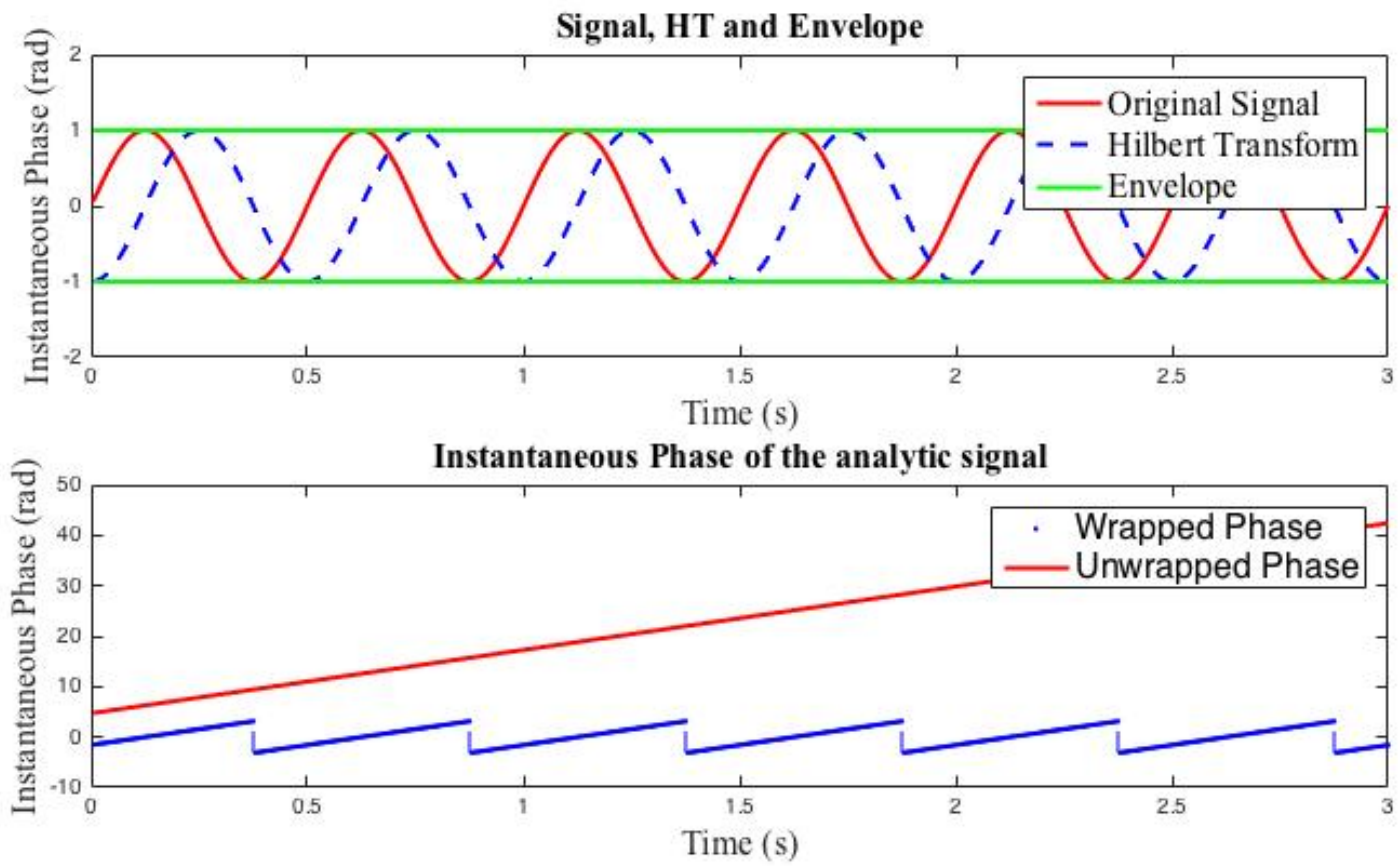

Figure 1.10. The HT, Envelope and the instantaneous phase 
The concept of Hilbert spectra analysis can be easily understand by showing the three-dimensional view of Hilbert spectrum as Figure 1.11. The moment, instantaneous frequency and the magnitude are all included in a single 3D Hilbert spectrum representation.

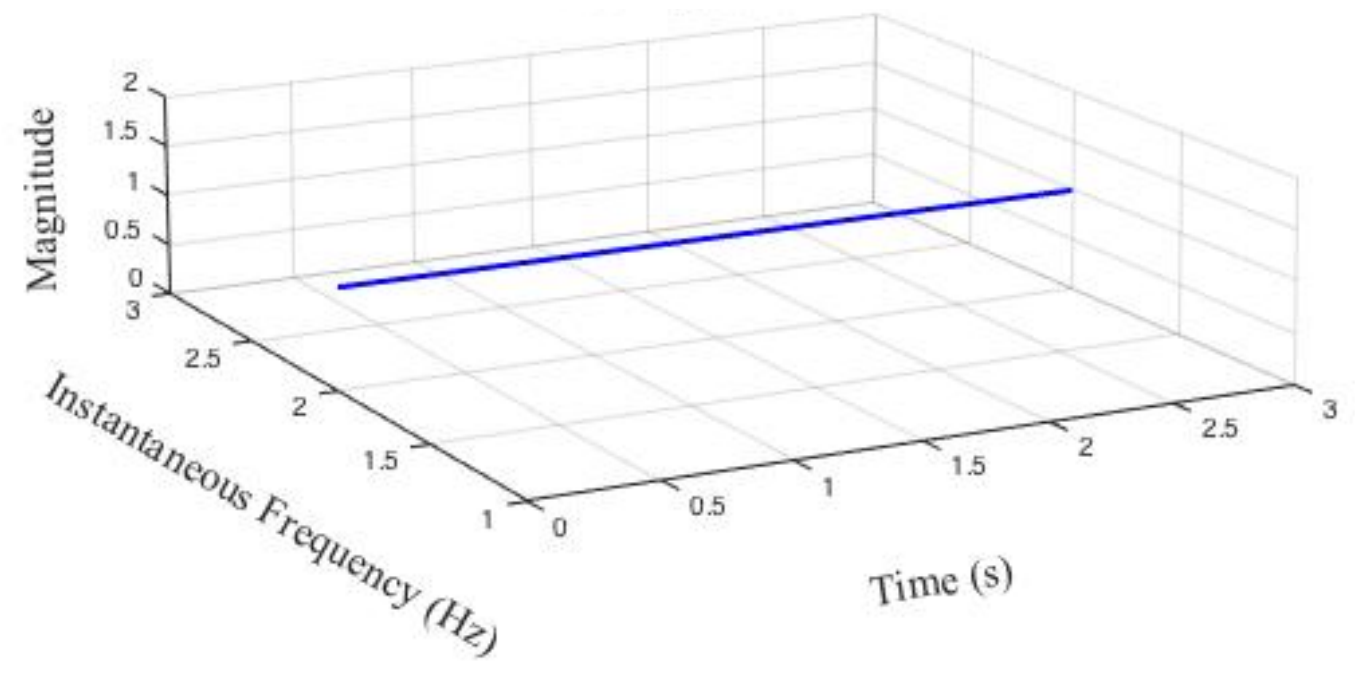

Figure 1.11. The 3-dimensoinal Hilbert spectrum

The time-frequency, frequency-magnitude and the time-magnitude plot can be considered as the projection of this 3D Hilbert spectrum onto the different plane. Since the original signal is a simple harmonic sine function, the frequency is a constant 2 in any time, as referred to Figure 1.12 (a). Moreover, the amplitude of the signal is a constant 1 , and it only exists at the constant frequency, as shown in Figure 1.12 (b) and (c). Note that the color of the line in time-frequency representation varies with the magnitude of the signal at the instant and the frequency, and it is still constant in this case. 


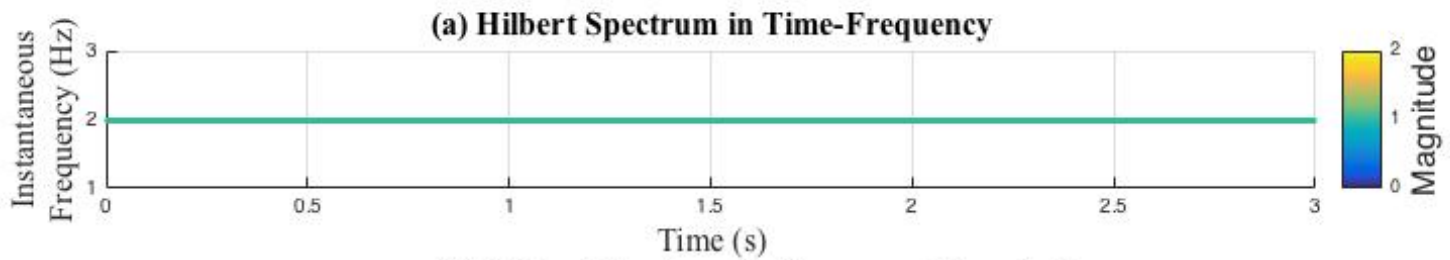

(b) Hilbert Spectrum in Frequency-Magnitude

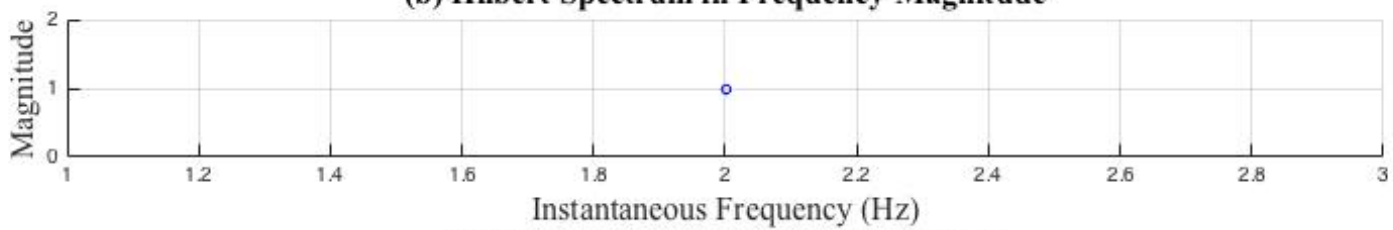

(c) Hilbert Spectrum in Time-Magnitude

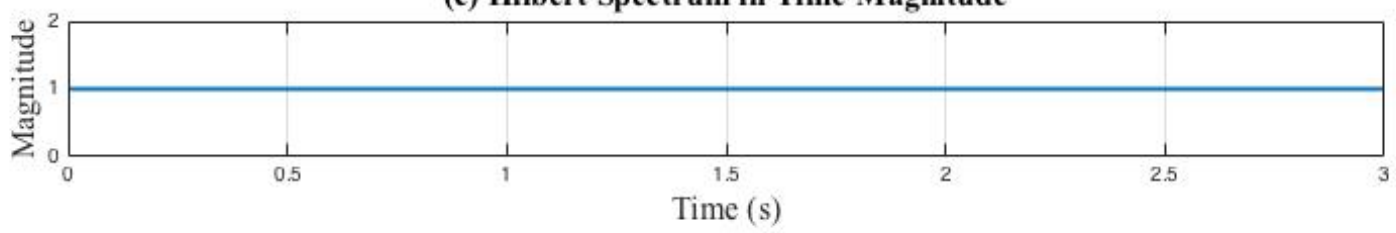

Figure 1.12. (a) The time-Frequency, (b) the Frequency-Magnitude,

and (c) the Time-Magnitude representation of the mono-component signal

\section{Example: Chirp Signal (Frequency Sweeping)}

A linear chirp signal is given as

$$
f(t)=w(t) \sin \left(2 \pi f_{0} t+\frac{\pi B t^{2}}{T}\right)
$$

Where $w(t)$ is the amplitude, $f_{0}$ is the starting frequency, $T$ is the duration of the chirp signal, and $B$ is the designed chirp bandwidth. The Hilbert Transform of the linear chirp signal is derived as

$$
\widehat{f}(t)=-w(t) \cos \left(2 \pi f_{0} t+\frac{\pi B t^{2}}{T}\right)
$$


The envelope of a chirp is simply its amplitude

$$
A(t)=\sqrt{[f(t)]^{2}+[\hat{f}(t)]^{2}}= \pm w(t)
$$

The instantaneous phase in the time domain is given by

$$
\varphi(t)=\tan ^{-1} \frac{\hat{f}(t)}{f(t)}=\tan ^{-1}\left[-\cot \left(2 \pi f_{0} t+\frac{\pi B t^{2}}{T}\right)\right]
$$

The instantaneous frequency is the phase derivative of time

$$
\omega(t)=\frac{d \phi(t)}{d t}
$$

The instantaneous frequency can be further computed as a constant as

$$
\omega(t)=\frac{d}{d t}\left\{\tan ^{-1}\left[-\cot \left(2 \pi f_{0} t+\frac{\pi B t^{2}}{T}\right)\right]\right\}=2 \pi\left(f_{0}+\frac{B t}{T}\right)
$$

For a linear chirp signal with initial frequency $f_{0}=50 \mathrm{kHz}$, bandwidth $B=300 \mathrm{kHz}$, and duration $T=200 \mu s$, the envelope, instantaneous phase and instantaneous frequency can be illustrated as figures below. Note that there are some artificial fluctuations due to end effects for numerical methods, analytic solutions are provided for comparison. 


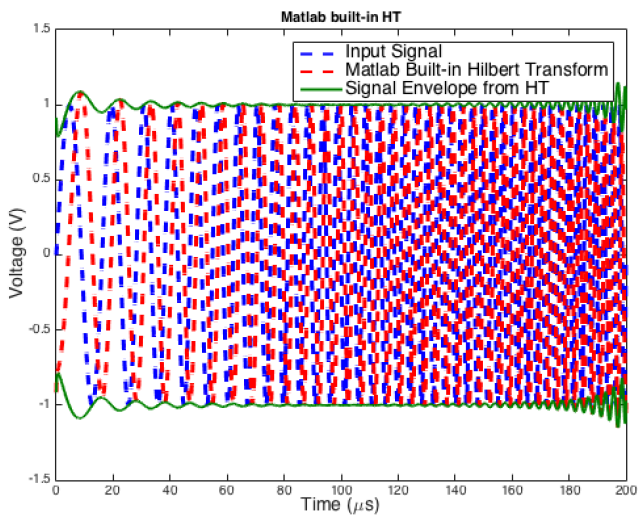

(a) Matlab built-in function

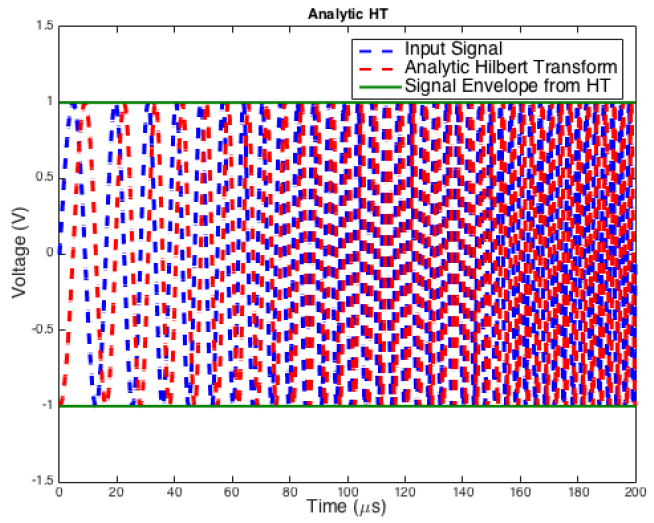

(b) Analytic solution

Figure 1.13. Chirp signal and its HT and envelope

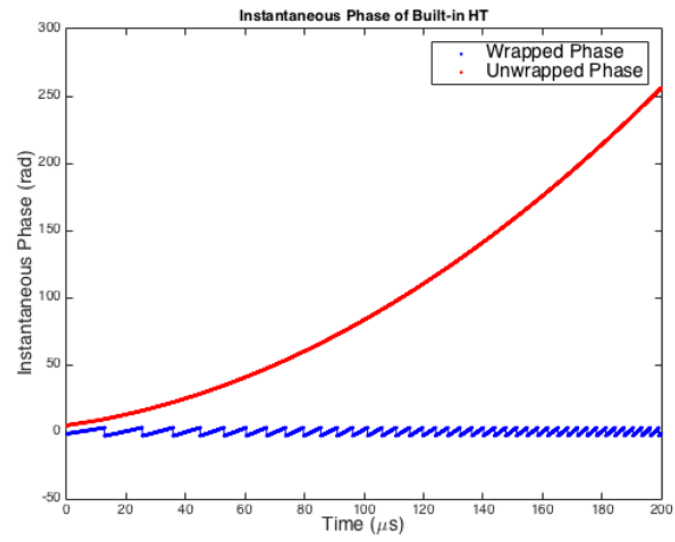

(a) Matlab built-in function

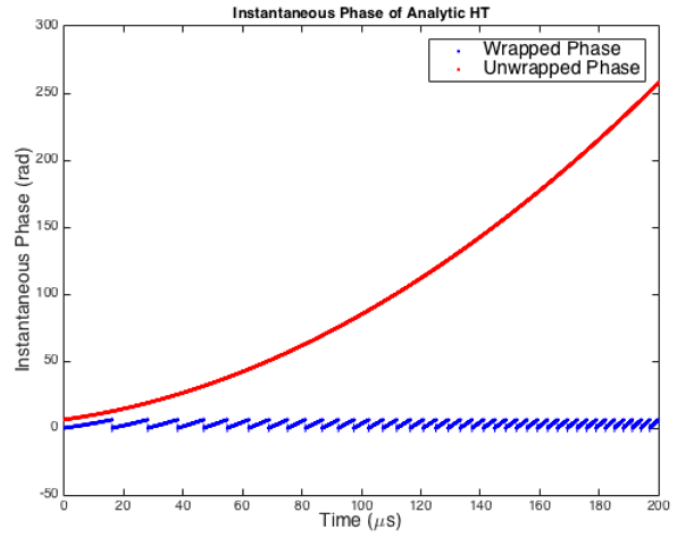

(b) Analytic solution

Figure 1.14. The instantaneous phase of Chirp signal from HT 


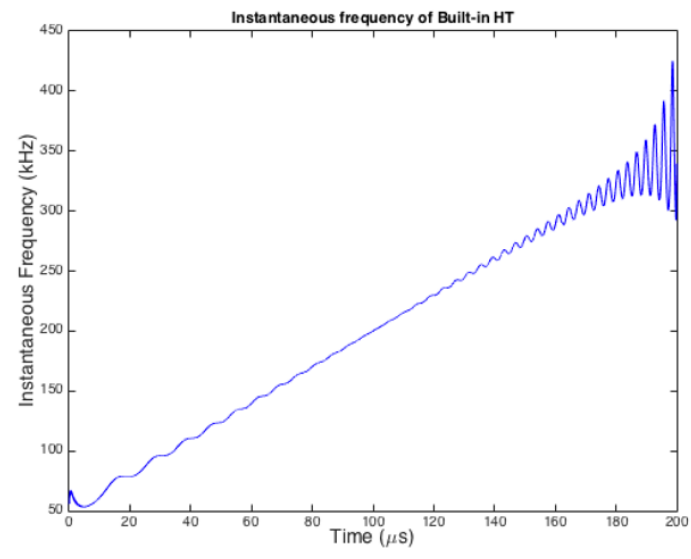

(a) Matlab built-in function

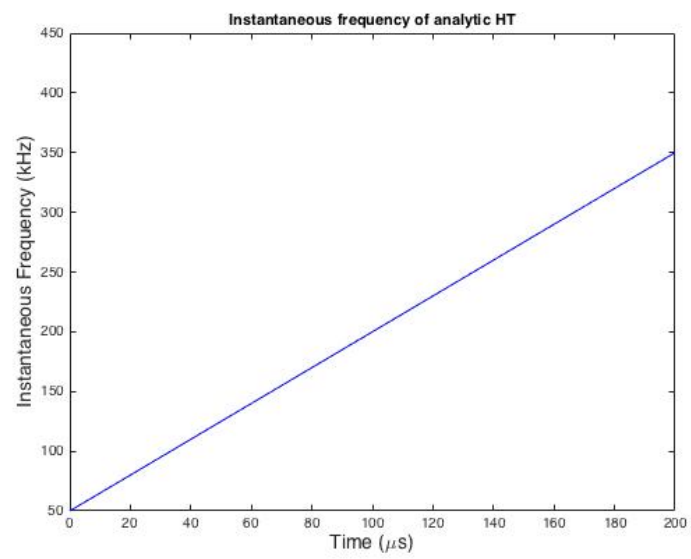

(b) Analytic solution

Figure 1.15. The instantaneous frequency of Chirp signal from HT

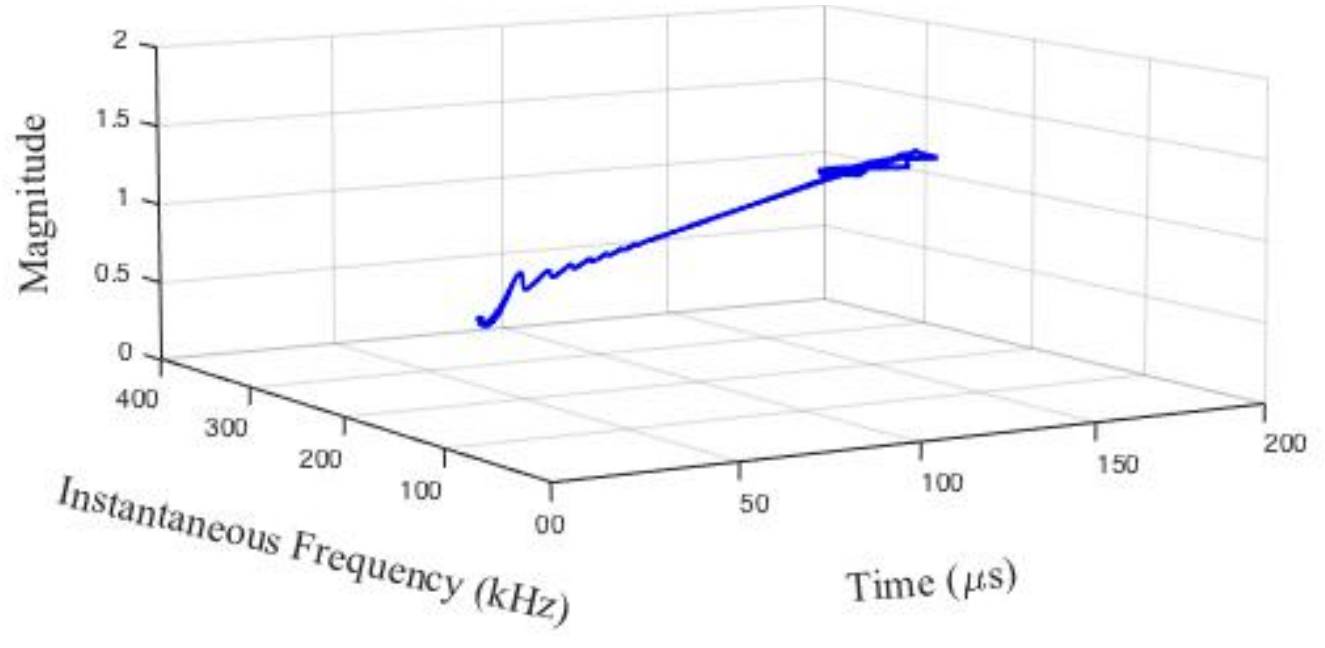

Figure 1.16. The 3-dimensoinal Hilbert spectrum 


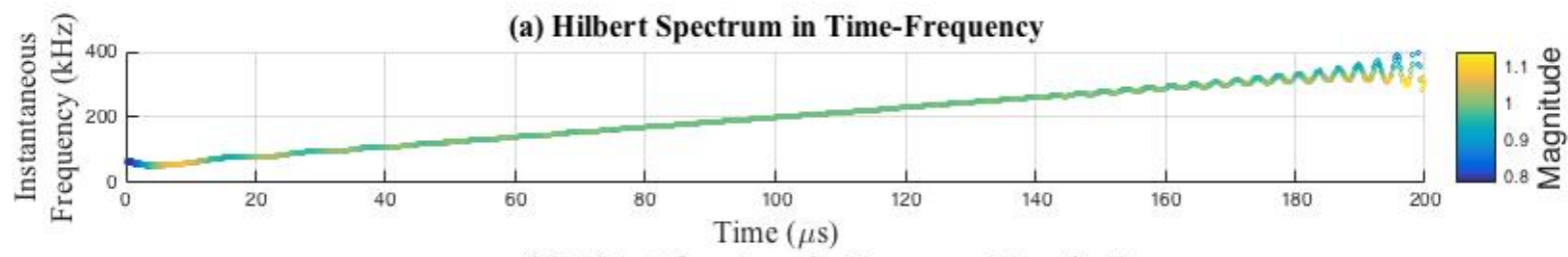

(b) Hilbert Spectrum in Frequency-Magnitude

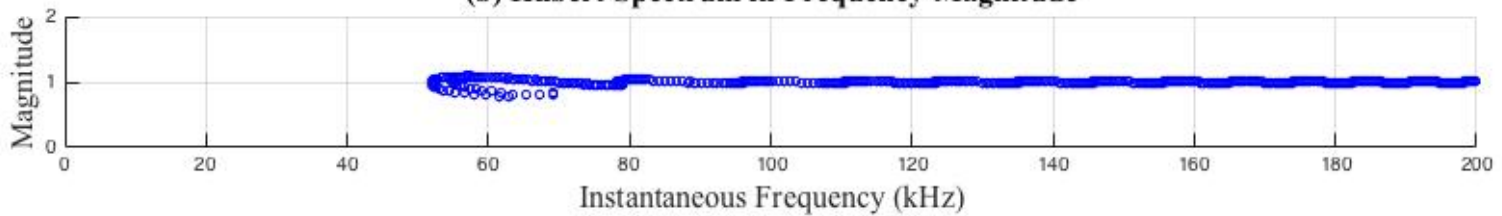

(c) Hilbert Spectrum in Time-Magnitude

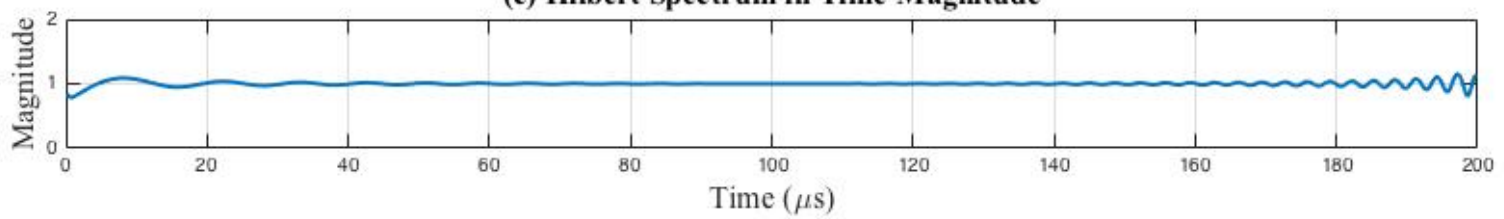

Figure 1.17. (a) The time-Frequency, (b) the Frequency-Magnitude, and (c) the Time-Magnitude representation of the linear chirp signal

\section{Example: Hanning-windowed Toneburst Signal}

The Hanning-modulated toneburst signal is a type of modulated signal comprehensively applied in structural health monitoring as an exciting source. Consider an n-cycle Hanning-modulated toneburst signal with a center frequency $f_{c}$ as

$$
f(t)=A\left[1-\cos \left(\omega_{c} t / n_{c}\right)\right] \sin \left(\omega_{c} t\right)
$$

where $n_{c}$ is the number of peaks. The Hilbert Transform of this n-cycle Hanning-modulated toneburst signal is

$$
\hat{f}(t)=-A\left[1-\cos \left(\omega_{c} t / n_{c}\right)\right] \cos \left(\omega_{c} t\right)
$$


As mentioned above, the signal envelope can be computed as

$$
A(t)= \pm A\left[1-\cos \left(\omega_{c} t / n_{c}\right)\right]
$$

Letting the central frequency $f_{c}=100 \mathrm{kHz}$, the input, Hilbert transform and the signal envelop of a 5-cycles Hanning-modulated toneburst signal are shown as below.

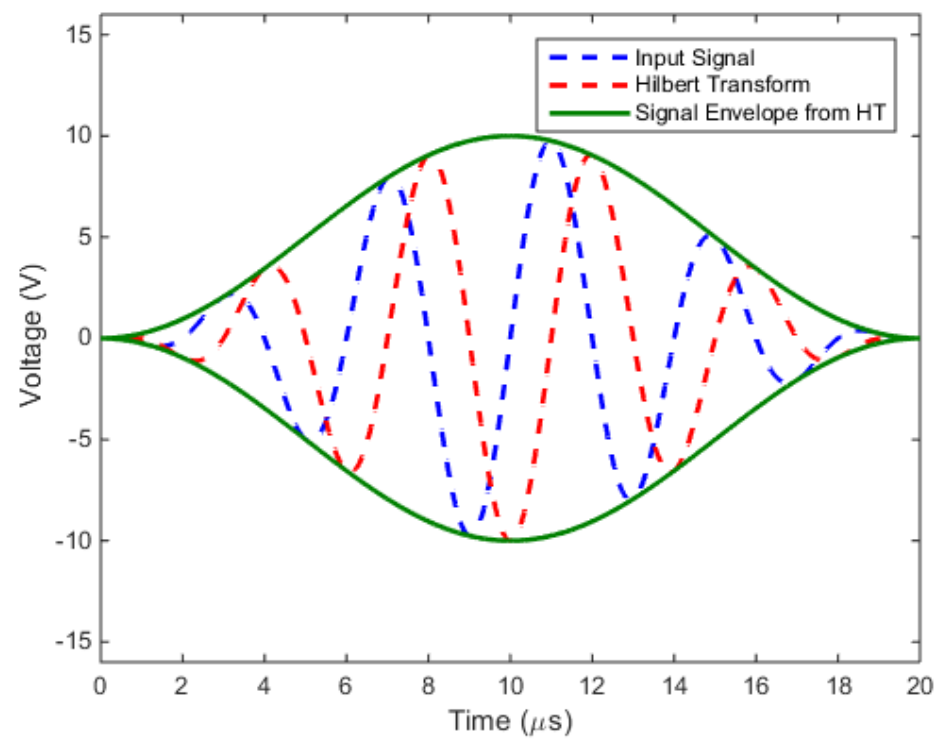

Figure 1.18. The Hilbert transform of a 5-cycle Hanning-modulated toneburst signal with a center frequency $100 \mathrm{kHz}$ and its envelope.

\section{Example: Modulated Signal}

The HT can be used for estimating modal parameters such as natural frequencies and damping ratios. For a SDOF (or single mode) system, the damped natural frequency can be easily determined from the HT of the impulse response function. In this case, the damped natural 
frequency $\omega_{d}$ is given by the slope of the instantaneous phase angle plotted as a function of time, and damping ratio is estimated in a straightforward manner.

For a linear SDOF system characterized by a pair of complex conjugate eigenvalues, the eigenvalues are directly related to the modal parameters as $-\zeta \omega_{n}=\sigma_{d}$, where $\omega_{n}$ denotes the natural frequency. The impulse response function is given by

$$
h(t)=\frac{1}{m \omega_{d}} e^{-\zeta \omega_{n} t} \sin \left(\omega_{d} t\right)
$$

where $\mathrm{m}$ is the mass. Provided that the damping ratio is small, the following relationships hold:

$$
\begin{aligned}
& \mathcal{H}\left[e^{-\zeta \omega_{n} t} \sin \left(\omega_{d} t\right)\right]=e^{-\zeta \omega_{n} t} \cos \left(\omega_{d} t\right) \\
& \mathcal{H}\left[e^{-\zeta \omega_{n} t} \cos \left(\omega_{d} t\right)\right]=e^{-\zeta \omega_{n} t} \sin \left(\omega_{d} t\right)
\end{aligned}
$$

Where $\mathcal{H}$ denotes the HT. Therefore the HT of the impulse response function can be written as

$$
\mathcal{H}[h(t)]=\hat{h}(t)=\frac{1}{m \omega_{d}} e^{-\zeta \omega_{n} t} \cos \left(\omega_{d} t\right)
$$

The analytic function is expressed by

$$
h_{+}(t)=\frac{1}{m \omega_{d}} e^{-\zeta \omega_{n} t} e^{i \omega_{d} t}
$$

The amplitude of the signal is

$$
A(t)=\frac{1}{m \omega_{d}} e^{-\zeta \omega_{n} t}
$$


which represents the envelope of the impulse response. Taking the natural logarithm of the amplitude yields

$$
\ln [A(t)]=-\zeta \omega_{n} t-\ln \left(m \omega_{d}\right)
$$

The slope $\sigma$ of the natural $\log$ of $A(t)$ versus gives $\sigma=-\zeta \omega_{n}$. Given that $\omega_{d}$ can be obtained from the slope of the phase versus time, and using the definition of the damped natural frequency, the natural frequency can be obtained.

$$
\begin{aligned}
& \omega_{d}=\sqrt{\left(1-\zeta^{2}\right) \omega_{n}} \\
& \omega_{d}^{2}=\left(1-\zeta^{2}\right) \omega_{n}=\omega_{n}^{2}-\sigma^{2} \\
& \omega_{n}=\sqrt{\omega_{d}^{2}+\sigma^{2}}
\end{aligned}
$$

and the damping ratio can be calculated as $\zeta-\sigma / \omega_{n}$. This approach to damping estimation is in the same spirit as the logarithm decrement technique commonly used in structural dynamics.

\subsubsection{Bandlimited signals}

All real physical processes, thus their representatives, the modulating and modulated signals, are bandlimited by nature. When such signals modulate a carrier signal, which frequency $\omega_{c}$ is typically much larger than the modulating signal spectral width, $W \ll \omega_{c}$, the modulated signal becomes narrowband. Since energy of the narrowband signal is concentrated around $\omega_{c}$, its major performance is the envelope, phase, and instantaneous frequency. An important property of bandlimited signals is that the amplitude $A(t)$ and phase $\theta(t)$ are slowly changing in time. 
A narrowband signal is defined as the bandwidth of the signal is a small fraction of the center frequency. The signal can be either amplitude modulated or frequency modulated. Usually the signal can be expressed in the form:

$$
s(t)=A(t) \cos \left[\omega_{c} t+\theta_{m}(t)\right]
$$

For AM signals,

$$
A(t)=A_{c}\left[1+k_{a} m(t)\right]
$$

where $m(t)$ is the baseband message, $c(t)=A_{c} \cos \omega_{c} t$ is called the carrier wave, The carrier frequency $f_{c}$, should be larger than the highest spectral component in $m(t)$. The parameter $k_{a}$ is a positive constant called the amplitude sensitivity of the modulator. The simplest AM is a single tone modulation, $m(t)=A_{m} \cos \omega_{m} t$, then Eq. (1.83) can be written as

$$
s(t)=A_{c}\left(1+\mu \cos \omega_{m} t\right) \cos \left(\omega_{c} t\right)
$$

For FM, the instantaneous frequency of an FM wave with carrier frequency $\omega_{c}$ for a baseband message $m(t)$ can be given by

$$
\omega(t)=\omega_{c}+k_{\omega} m(t)
$$

where $k_{\omega}$ is a positive constant called the frequency sensitivity. The angle of the FM signal, assuming the value is zero at $t=0$, is

$$
\theta(t)=\int_{0}^{t} \omega(\tau) d \tau=\omega_{c} t+\theta_{m}(t)
$$

where 


$$
\theta_{m}(t)=k_{\omega} \int_{0}^{t} m(\tau) d \tau
$$

is the carrier phase deviation caused by $m(t)$.

The FM signal generated by $m(t)$ is

$$
s(t)=A_{c} \cos \left[\omega_{c} t+\theta_{m}(t)\right]
$$

Let the same single tone modulation function be given by $m(t)=A_{m} \cos \omega_{m} t$, then

$$
s(t)=A_{c} \cos \left(\omega_{c} t+\frac{k_{\omega} A_{m}}{\omega_{m}} \sin \omega_{m} t\right)
$$

where modulation index $\beta$ is defined as

$$
\beta=\frac{k_{\omega} A_{m}}{\omega_{m}}=\frac{\text { peak frequency deviation }}{\text { modulating frequency }}
$$

In the case where $\left|\theta_{m}(t)\right| \ll 1$ for all $\mathrm{t}$ is called narrowband FM. Using the approximations $\cos x \cong 1$ and $\sin x \cong x$, the FM signal can be approximated as

$$
\begin{aligned}
& s(t)=A_{c} \cos \left[\omega_{c} t+\theta_{m}(t)\right] \\
& =A_{c} \cos \omega_{c} t \cos \theta_{m}(t)-A_{c} \sin \omega_{c} t \sin \theta_{m}(t) \\
& \simeq A_{c} \cos \omega_{c} t-A_{c} \theta_{m}(t) \sin \omega_{c} t
\end{aligned}
$$

or in complex form

$$
s(t) \simeq A_{c} \operatorname{Re}\left\{e^{i \omega_{c} t}\left[1+i \theta_{m}(t)\right]\right\}
$$


This form is similar to the AM signal except that the discrete carrier component $A_{c} \cos \omega_{c} t$ is $90^{\circ}$ out of phase with the sinusoid $A_{c} \sin \omega_{c} t$ multiplying the phase angle $\theta_{m}(t)$. The spectrum of narrowband FM is similar to AM. The analytic signal can be expressed by

$$
S_{+}(t)=A(t) e^{i \theta_{m}(t)} e^{i \omega_{c} t}
$$

The instantaneous frequency is

$$
\frac{1}{2 \pi}\left(\omega_{c}+\frac{d \theta_{m}}{d t}\right)
$$

A breakthrough of this method is that as opposed to the traditional time-frequency analysis techniques, it does not use pre-specified basis functions or filters but instead decomposes a signal by direct extraction of the local energy associated with the intrinsic time scales of the signal itself. It is therefore highly adaptive and consequently can well depict the time-frequency characteristics of a signal [2.13]. The excitation signal in SHM is often decomposed as a low-frequency modulating signal that acts as modulator of a high-frequency carrier signal. In industrial applications such as gear fault detection, the low-frequency modulating signal is the gear rotation frequency and the high-frequency carrier is the meshing frequency. Amplitude and phase modulations can be extracted by Hilbert transform [2.14]. 


\subsubsection{Properties}

Giving real functions $s_{n}(t)$, its Hilbert transform can be expressed as $\hat{s}_{n}(t)=\mathcal{H}\left(s_{n}(t)\right)$, where $n$ can be any integer. Some basic properties of the Hilbert transform are provided below, noting that $a, a_{1}$ and $a_{2}$ are arbitrary constants [2.9][2.15].

Table 1.4. Properties of Hilbert transform

\begin{tabular}{ll}
\hline \hline Name & Property \\
\hline \hline Linearity & $\mathcal{H}\left(a_{1} s_{1}(t)+a_{2} s_{2}(t)=a_{1} \mathcal{H}\left(s_{1}(t)\right)+a_{2} \mathcal{H}\left(s_{2}(t)\right)\right.$ \\
\hline Time shift & $\mathcal{H}(s(t-a))=\hat{s}(t-a)$ \\
\hline Scaling & $\mathcal{H}(s(a t))=\hat{s}(a t), a>0$ \\
\hline Derivative & $\mathcal{H}\left(s^{\prime}(t)\right)=\hat{s}^{\prime}(t)$ \\
\hline Time reversal & $\mathcal{H}(s(-a t))=-\hat{s}(-a t)$ \\
\hline Inversion & $\mathcal{H} \mathcal{H}(s(t))=-s(t)$ \\
\hline Orthogonality & $\int_{-\infty}^{\infty} s(t) \cdot \mathcal{H}(s(t)) d t=0$ \\
\hline Convolution & $\mathcal{H}\left[s_{1}(t) * s_{2}(t)\right]=\hat{s}_{1}(t) * s_{2}(t)=s_{1}(t) * \hat{s}_{2}(t)$ \\
\hline Multiplication by t & $\mathcal{H}(t s(t))=t \hat{s}(t)-\int_{-\infty}^{\infty} s(\tau) d \tau$
\end{tabular}

Bedrosian's theorem states that the Hilbert transform of the product of a low-pass and a high-pass signal with non-overlapping spectra is given by the product of the low-pass signal and the Hilbert transform of the high-pass signal [2.16]or

$$
\mathcal{H}\left[f_{L P}(t) f_{H P}(t)\right]=f_{L P}(t) \mathcal{H}\left[f_{H P}(t)\right]
$$


Where $f_{L P}$ and $f_{H P}$ are the low- and high-pass signals respectively [2.17] Amplitude modulated signals are modeled as the product of a bandlimited "message" waveform, $u_{m}(t)$, and a sinusoidal "carrier":

$$
u(t)=u_{m}(t) \cos (\omega t+\phi)
$$

When $u_{m}(t)$ has no frequency content above the carrier frequency, $\frac{\omega}{2 \pi} \mathrm{Hz}$, then by Bedrosian's theorem :

$$
H[u(t)]=u_{m}(t) \sin (\omega t+\phi)
$$

For modulated signals for excitation, amplitude modulated signal in particular, Bedrosian's theorem provides a convenient way to obtain the HT, sometimes in an approximate way. The theorem is stated as follows

Suppose $f(\omega)$ and $g(\omega)$ have Fourier transform $\tilde{f}(\omega)$ and $\tilde{g}(\omega)$ respectively. If $\tilde{f}(\omega)=0$ for $|\omega|>a$ with $a>0$ and $\tilde{g}(\omega)=0$ for $|\omega|<a$. Then

$$
\mathcal{H}(f(t) g(t))=f(t) \mathcal{H}(g(t))
$$

In general case where the two signals have overlapped spectra, then the HT can be written in the form of a sum of two parts.

$$
\begin{aligned}
\mathcal{H}[n(t) x(t)] & \left.=\mathcal{H}\left\{\bar{n}(t)+n_{1}(t)\right] x(t)\right] \\
& =\bar{n}(t) \hat{x}(t)+\hat{n}_{1}(t) x(t)
\end{aligned}
$$


where $\bar{n}(t)$ is the slow (low-pass), $n_{1}(t)$ is the fast (high-pass) signal component, and $\hat{n}_{1}(t)$ is the HT of the fast component.

Some useful properties of the Hilbert transform pair are listed follow:

$$
\begin{gathered}
\int_{-\infty}^{\infty} x(t) \hat{x}(t) d t=0 \\
\int_{-\infty}^{\infty} x^{2}(t) d t=\int_{-\infty}^{\infty} \hat{x}^{2}(t) d t \\
d \hat{x}(t) / d t=\mathcal{H}(d x(t) / d t) \\
\hat{x}(a t+b)=\mathcal{H}[x(a t+b)] \\
\mathcal{H}[x(t) * \hat{x}(t)]=\mathcal{H}[x(t)] * \hat{x}(t)+x(t) * \mathcal{H}[\hat{x}(t)]
\end{gathered}
$$

The approximate relations follow:

$$
\begin{gathered}
\mathcal{H}\left[a(t) \cos \left(\omega_{c} t+\phi\right)\right] \cong-a(t) \sin \left(\omega_{c} t+\phi\right) \\
\mathcal{H}\left[\exp \left(-\omega_{c} t / \gamma\right)^{2} \cos \left(\omega_{c} t+\phi\right)\right] \cong-\exp \left(-\omega_{c} t / \gamma\right)^{2} \sin \left(\omega_{c} t+\phi\right)
\end{gathered}
$$

Where $a(t)$ is a slowly-varying envelope of the harmonic carrier with frequency $\omega_{c}$. Eq.(1.106)(a) shows the Hilbert transform of Gabor signal as a special case of Eq. (1.106)(a). For Eq. (1.106)(b) to be sufficiently accurate, $\gamma$ ought to be selected greater than 3 . 


\subsection{Hilbert-Huang Transform}

\subsubsection{Empirical Mode Decomposition and Hilbert Spectrum Analysis}

Hilbert Transform (HT) is a great tool for time-frequency analysis. However, it has limitation to narrow band signals, which leads to less practicability for real-world signals. In order to apply HT to wideband or multi-component signals, Huang et al. proposed an improved time-frequency analysis methods named Hilbert-Huang Transform (HHT), which consists two parts: Empirical Mode Decomposition (EMD) and Hilbert Spectral Analysis (HSA). In contrast to most of previous signal processing methods, HHT is not prior-based, and it is intuitive and adaptive, leading to great performance for handling non-stationary and non-linear signals. The HHT uses Empirical Mode Decomposition (EMD) to decompose the signal into multiple mono-component signals, defined as Intrinsic Mode Function (IMF), and then HT can be effectively applied to each IMF at any instant in time. The whole idea of HHT is basically based on the EMD which decomposes the dataset into IMFs. The EMD can be considered as a "sifting" procedure, gradually sifting out each IMF representing its intrinsic oscillation from high to low. The IMF can be considered as the generalized simple harmonic function, which has a variable amplitude and frequency as function of time. An IMF is defined as the function that satisfies the two following conditions [2.18]:

i. Over its entire dataset, the number of extrema and the number of zero crossings must either equal or differ at most by one.

ii. At any point, the mean value of the envelope defined by the local maxima and the local minima is zero. 
For the EMD to be applicable, it is assumed that

i. The signal has at least one maximum and one minimum.

ii. The characteristic timescale is defined by the lapse between the extrema.

If the data are totally devoid of extrema but contain only inflection points, then it can be differentiated one or more times to reveal the extrema.

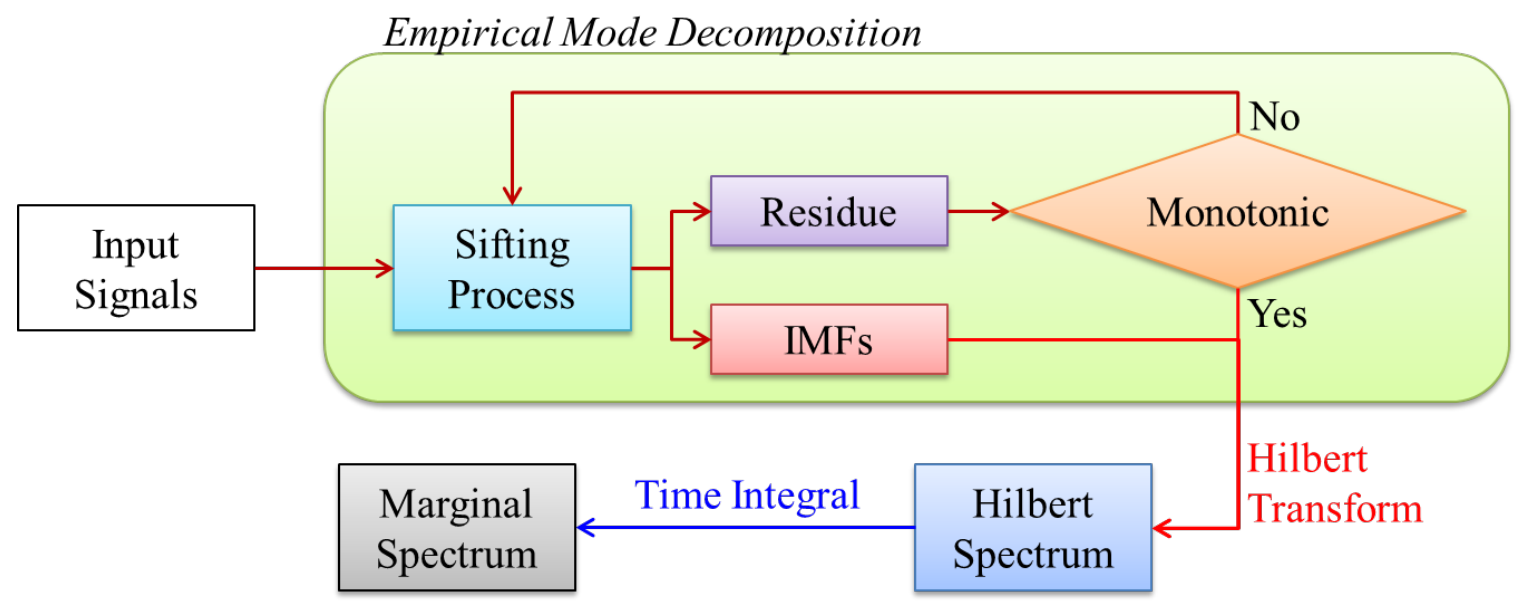

Figure 1.19. The process of HHT

Given a continuous time series dataset $s(t)$, the EMD procedure can be illustrated as follows. First letting the initial component $h_{10}(t)$, for convenience, the original data is assumed as the initial residue $r_{0}(t)$.

$$
h_{10}(t)=r_{0}(t)=s(t)
$$


For better understanding, the example is provided below,

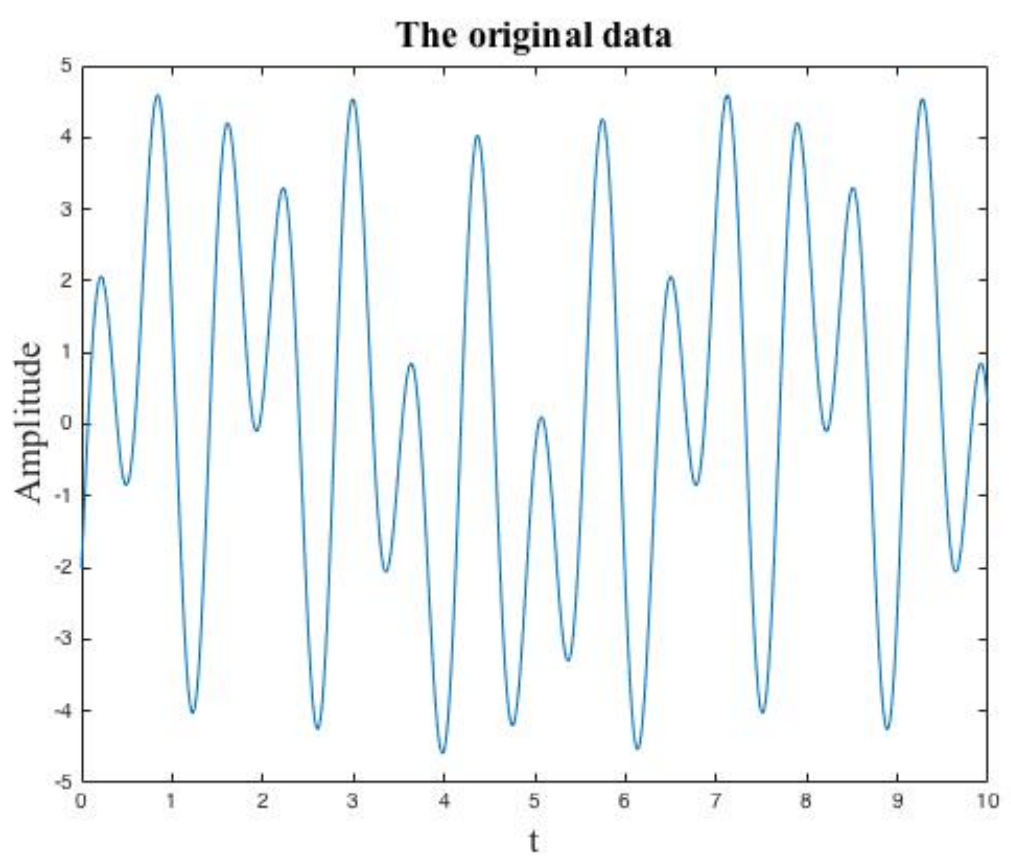

Figure 1.20. The original data

Extract the local maxima and local minima to generate upper and lower envelopes using spline interpolation, and then derive the mean $m_{11}(t)$ of the two envelopes. The first component $h_{11}(t)$ of the first IMF is computed as the difference between the input data and the mean as

$$
h_{11}(t)=h_{10}(t)-m_{10}(t)
$$




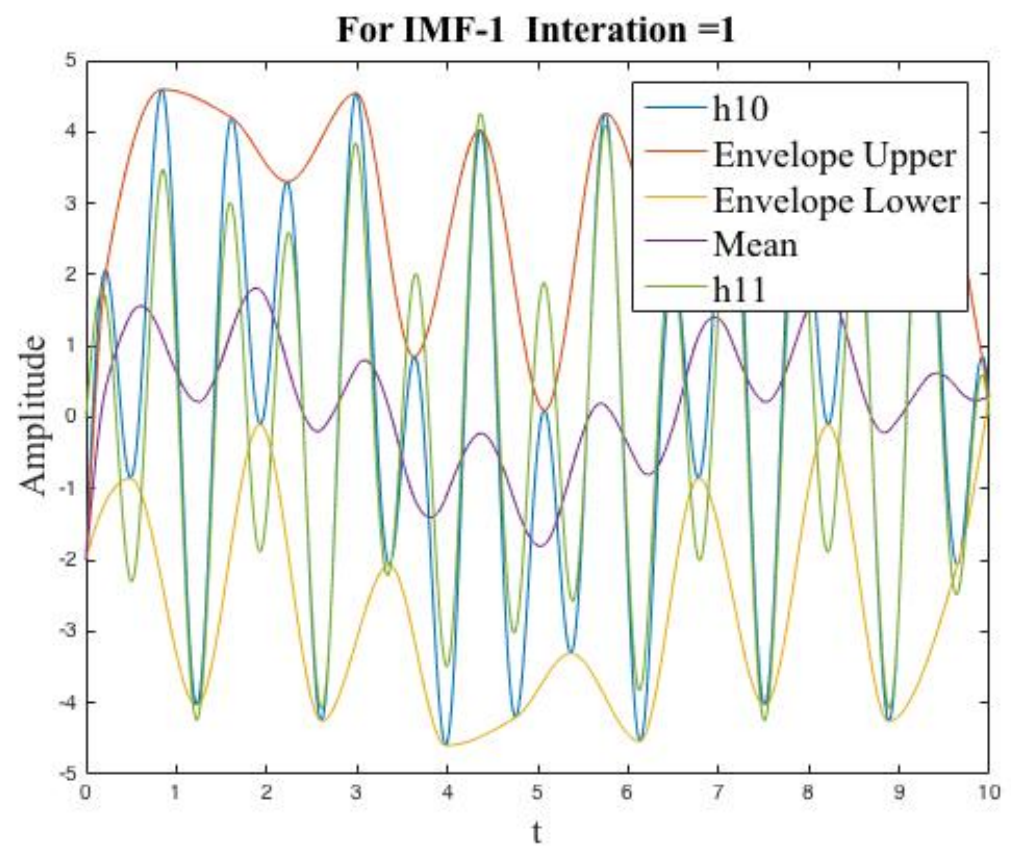

Figure 1.21. The first component $h_{11}$ of the first IMF

The envelopes are slowly varying compared with the fine time-scale. This means that when performing the sifting the low frequency components are being removed and the high frequency signal is derived. This shows why the IMFs appear in a high to low frequency order and why the EMD is essentially a time-frequency decomposition method and behaves like a bank of filters.

The component derived here should satisfy the definition of the IMF mentioned above. This sifting process not only provides the availability for HT to compute meaningful instantaneous frequencies, but also eliminates the disparity between neighboring wave amplitudes. Repeatedly operate the sifting process until the signal reaches designated stopping criteria. As repeat the sifting process for $k$ times, the kth component $h_{1 k}(t)$ is then the first IMF $c_{1}(t)$.

$$
c_{1}(t)=h_{1 k}(t)=h_{1(k-1)}(t)-m_{1(k-1)}(t)
$$




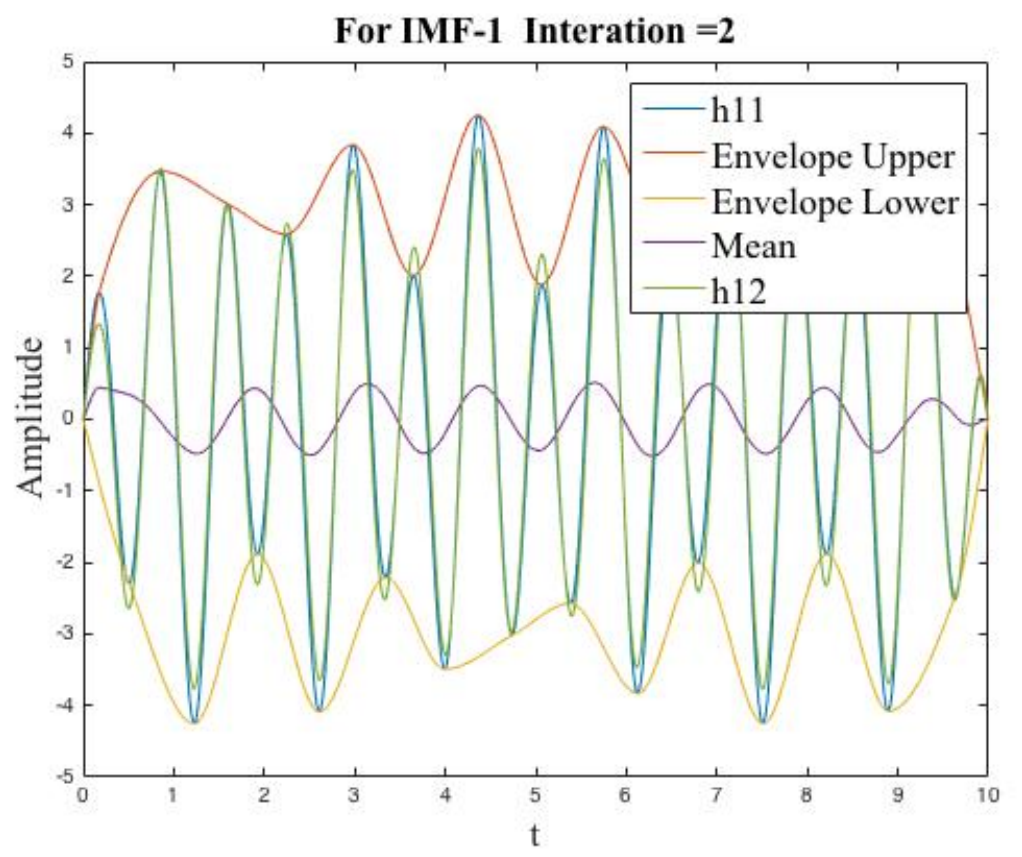

Figure 1.22. The second component $h_{12}$ of the first IMF

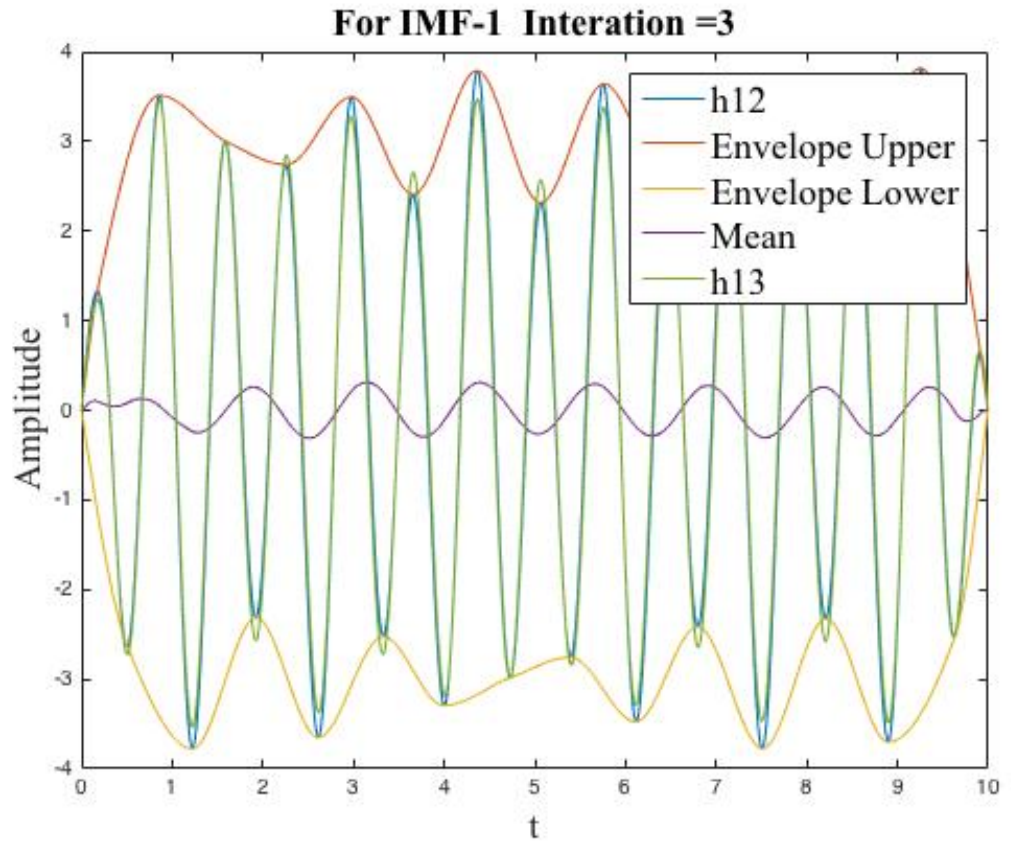

Figure 1.23. The third component $h_{13}$ of the first IMF 


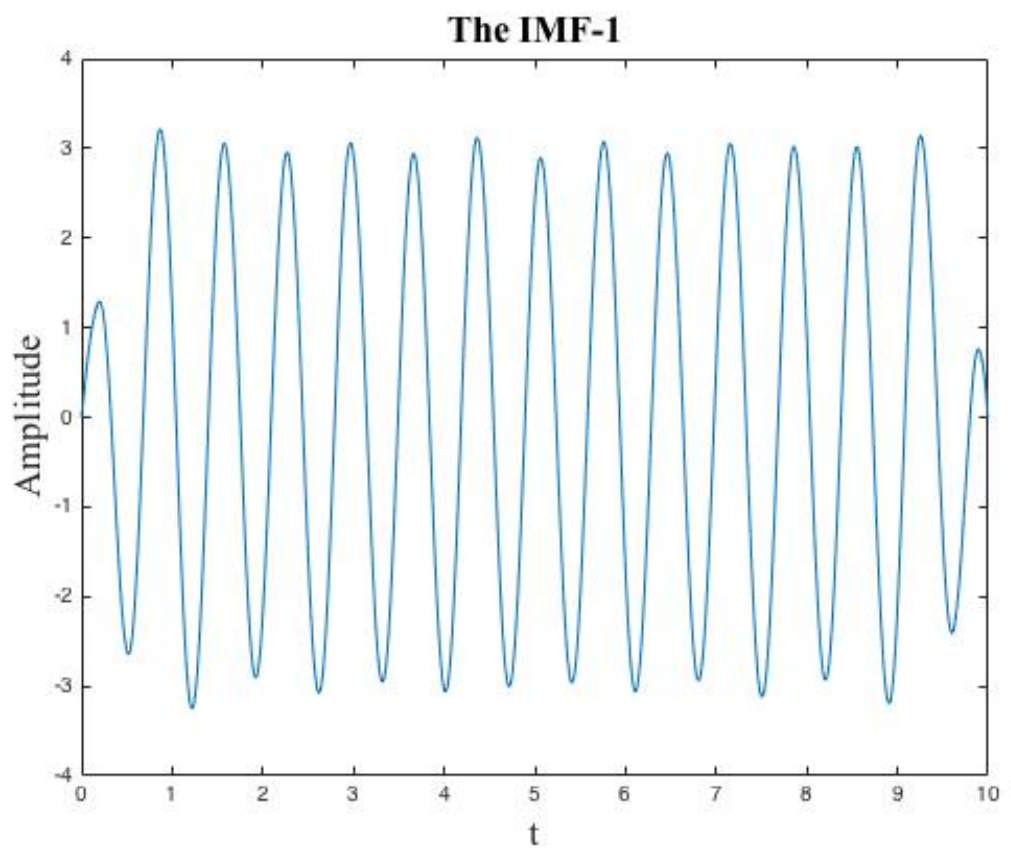

Figure 1.24. The first IMF after 6 iterations

The residue of the first IMF $r_{1}(t)$ is defined as the difference between the input data and the derived IMF,

$$
r_{1}(t)=s(t)-c_{1}(t)
$$




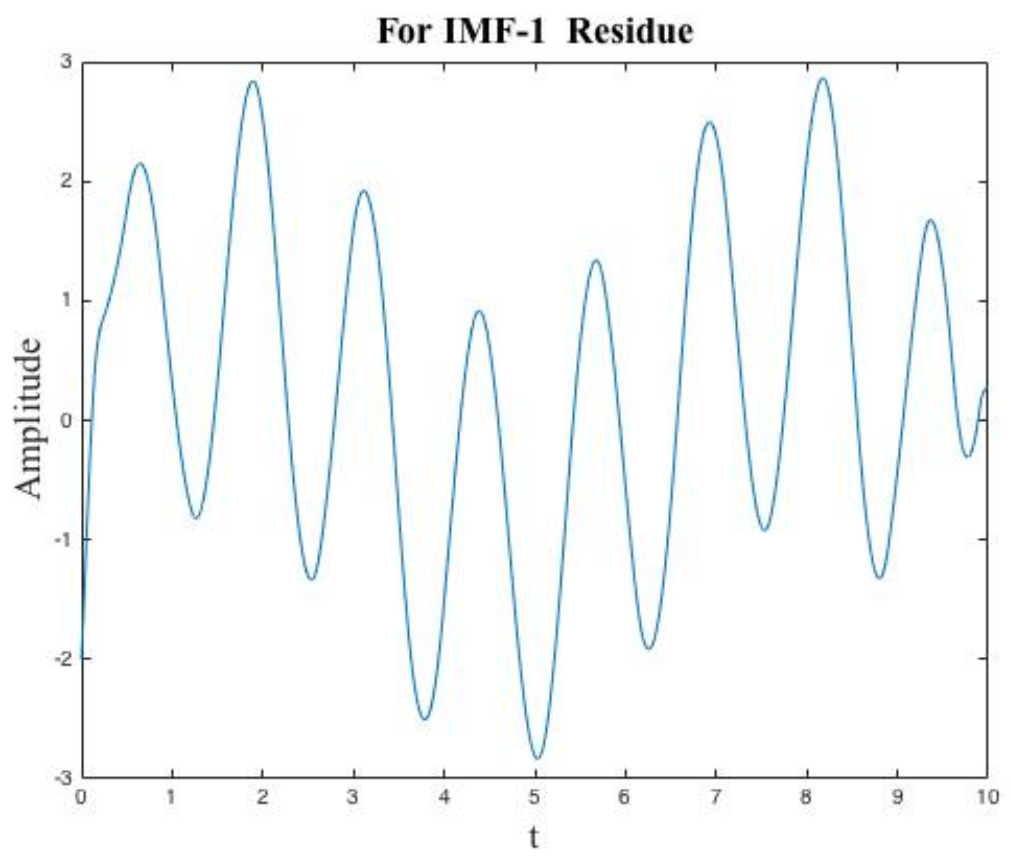

Figure 1.25. The residue of first IMF

The stopping criterion is very important here in the sifting process. There are several different definitions, and the first and the most basic one is proposed by Huang et al. [2.18], which is defined by using the Cauchy convergence test, requiring the normalized squared difference between successive components derived during sifting process to be smaller than the designated value.

$$
\mathrm{SD}_{j}=\sum_{t=0}^{T}\left(\frac{\left|h_{j(k-1)}(t)-h_{j k}(t)\right|^{2}}{h_{j(k-1)}^{2}(t)}\right)
$$

Where $T$ is the entire time length of the data, $j$ is the number of the IMF.

The problem of this predetermined stopping value is that it is difficult to pick a value which is small enough for sifting, but not too small. If the value is not properly decide, either the IMF derived cannot be representative or the artificial component will be generated. Moreover, even though the EMD itself is adaptive, the whole decomposition will be limited with the predetermined 
value, leading to shortcomings of current method. For overcoming the problem of this stopping criterion, some other new definitions are proposed afterwards, but there are still issues for applying in practice.

The residue of the first IMF is then the initial input data for the sifting process for second IMF. The sifting can be repeated until either the component derived and the residue is too small, or there is no more IMF to be found when the signal becomes a monotonic function. The last residue should be either a constant or the trend of the signal. After the sifting process stop, the original signal can be represented as

$$
s(t)=\sum_{j=1}^{n} c_{j}(t)+r_{n}
$$

where $\mathrm{n}$ is the total amount of IMFs derived. 
The HHT algorithm can be summarized in the Table 1.5.

Table 1.5. The HHT algorithm

1) Initialize: $r_{0}=\mathrm{s}(t)$, and $j=1$
2) Extract the $j^{\text {th }}$ IMF by using EMD
a) Initialize: $h_{j(k-1)=r_{j}, k=1}$
b) Extract the local extremes and minima of $h_{j(k-1)}$
c) Interpolate the local extrema and the minima by cubic splines to form upper and lower
envelopes of $h_{j(k-1)}$
d) Calculate the mean $m_{j(k-1)}$ of the upper and lower envelopes of $h_{j(k-1)}$
e) Let $h_{j k}==h_{j(k-1)}-m_{j(k-1)}$
f) If $h_{j k}$ is a IMF then set $c_{j}=h_{j k}$ else go to step (b) with $k=k+1$
3) Define $r_{j+1}=r_{j}-c_{j}$
4) If $r_{j+1}$ still has least 2 extremes the go to step (2), else decomposition processing is done and
$r_{j+1}$ is the residue of the given signal
5) Perform HT for each IMF and then plot Hilbert spectrum $H(\omega, t)$

After the empirical mode decomposition process, all the intrinsic mode function should be found. Hilbert transform can now be effectively applied to each IMF for obtaining instantaneous frequency, and the original data can be expressed as the real part of the summation of the analytic signal for each IMF. After the decomposition, either $r_{n}(t)$ is a constant, then $\mathcal{H}\left[r_{n}(t)\right]=0$, or it is monotonic. If $r_{n}(t)$ is monotonic, then it can potentially overpower the harmonics and should therefore be left out, so the residue term $r_{n}(t)$ will be disregarded here.

From the analytic expression of $s_{+}(t)$ along with the decomposition of $s(t)$ through HHT, one gets 


$$
\begin{aligned}
s_{+}(t) & =\sum_{j=1}^{n} c_{j}(t)+i \mathcal{H}\left(\sum_{j=1}^{n} c_{j}(t)\right) \\
& =\sum_{j=1}^{n}\left[c_{j}(t)+i \mathcal{H} c_{j}(t)\right] \\
& =\sum_{j=1}^{n} a_{j}(t) e^{i \phi_{j}(t)} \\
& =\sum_{j=1}^{n} a_{j}(t) e^{i\left[\phi(0)+\int_{0}^{t} \omega_{j}(\tau) d \tau\right.}
\end{aligned}
$$

Thus, another way of representing the time series $s(t)$ is given by

$$
s(t)=\operatorname{Re}\left(\sum_{j=1}^{n} a_{j}(t) e^{i\left[\phi(0)+\int_{0}^{t} \omega_{j}(\tau) d \tau\right.}\right)
$$

Now if $s(t)$ were to expand it into a Fourier representation, the representation in terms of complex exponential can be written as

$$
s(t)=\sum_{j=-\infty}^{\infty} a_{j} e^{-i \omega_{j} t}
$$

Obviously, these expressions seemingly look similar, but differ in many important ways. In the above expression, $a_{j}$ and angular frequencies $\omega_{j}$, are constant while the corresponding terms are time-variant. Thus the HHT with its decomposition can be viewed as a generalized Fourier series expansion. The time-varying amplitudes and frequencies yield to much better representation of nonstationary and nonlinear signals [2.15].

For example, given a multi-component signal which is composed of three sinusoidal functions with three distinguish frequencies and one linear function as

$$
f(t)=t+\sin 2 \pi t+\cos 10 \pi t+\sin 20 \pi t
$$


and the multi-component signal is also shown in Figure 1.26.

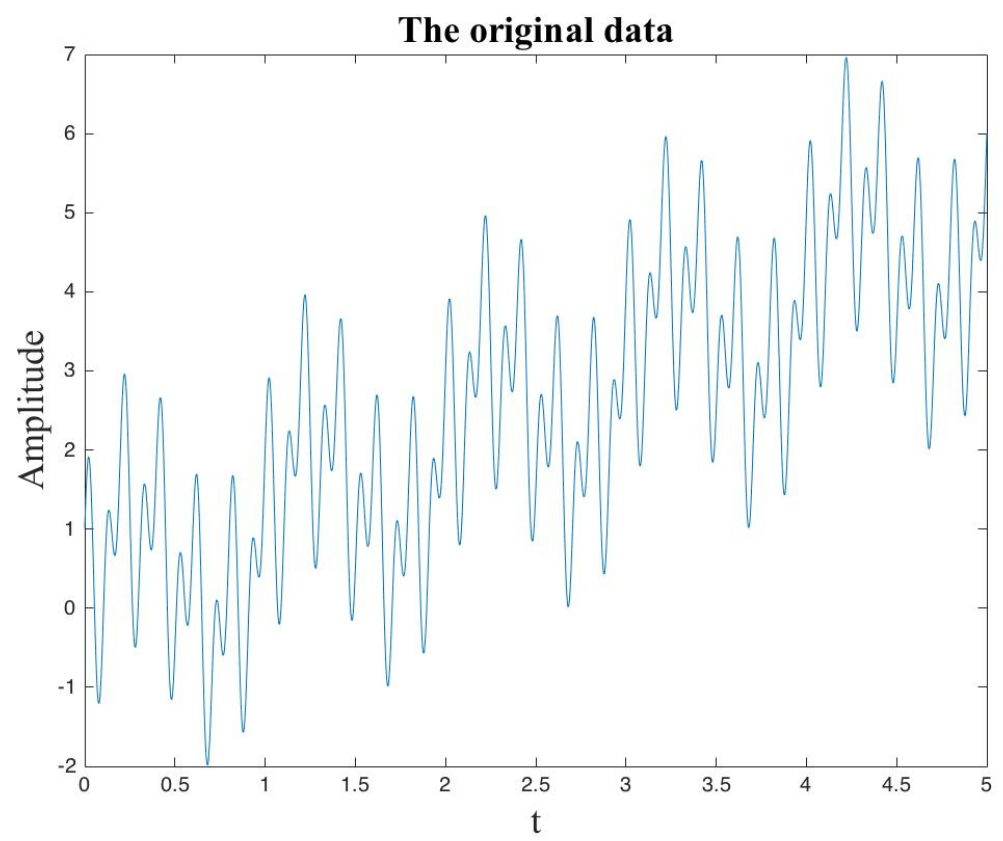

Figure 1.26. The multi-component signal in time domain

The signal consists of three frequencies, and it can be known by applying a fast Fourier transform and removing the DC component, as shown in Figure 1.27. Even though Hilbert transform can provide good resolution in time-frequency analysis and is effective for nonstationary and nonlinear signal, it is hard to get useful information directly from the Hilbert spectrum of the multicomponent signals. The Hilbert spectrum of the signal is also shown in Figure 1.28 (DC terms have been removed).

The multi-component signal consists of three IMFs, theoretically, which are $c_{1}=\sin 20 \pi t$, $c_{2}=\cos 10 \pi t$ and $c_{3}=\sin 2 \pi t$, and the theoretical residue is $r=t$. By applying the EMD method mentioned above to the signal, three IMFs and the residue can be derived numerically and are shown in Figure 1.29 along with the theoretical result for comparison. 


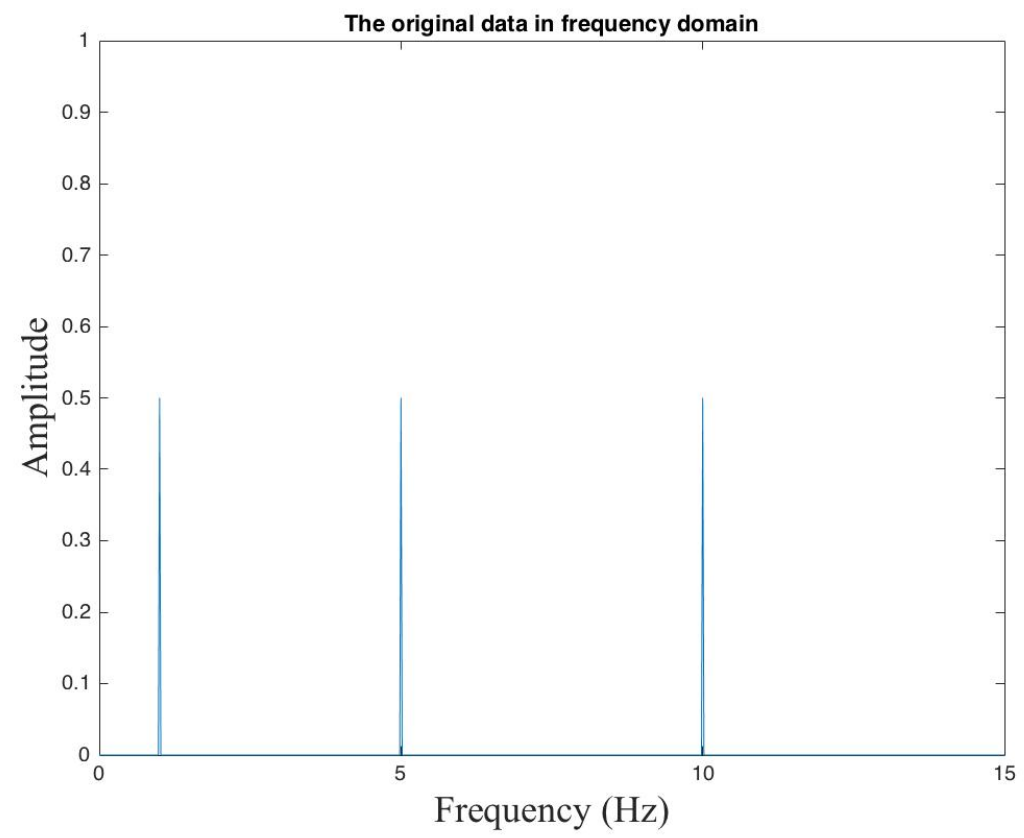

Figure 1.27. The FFT of the multi-component signal

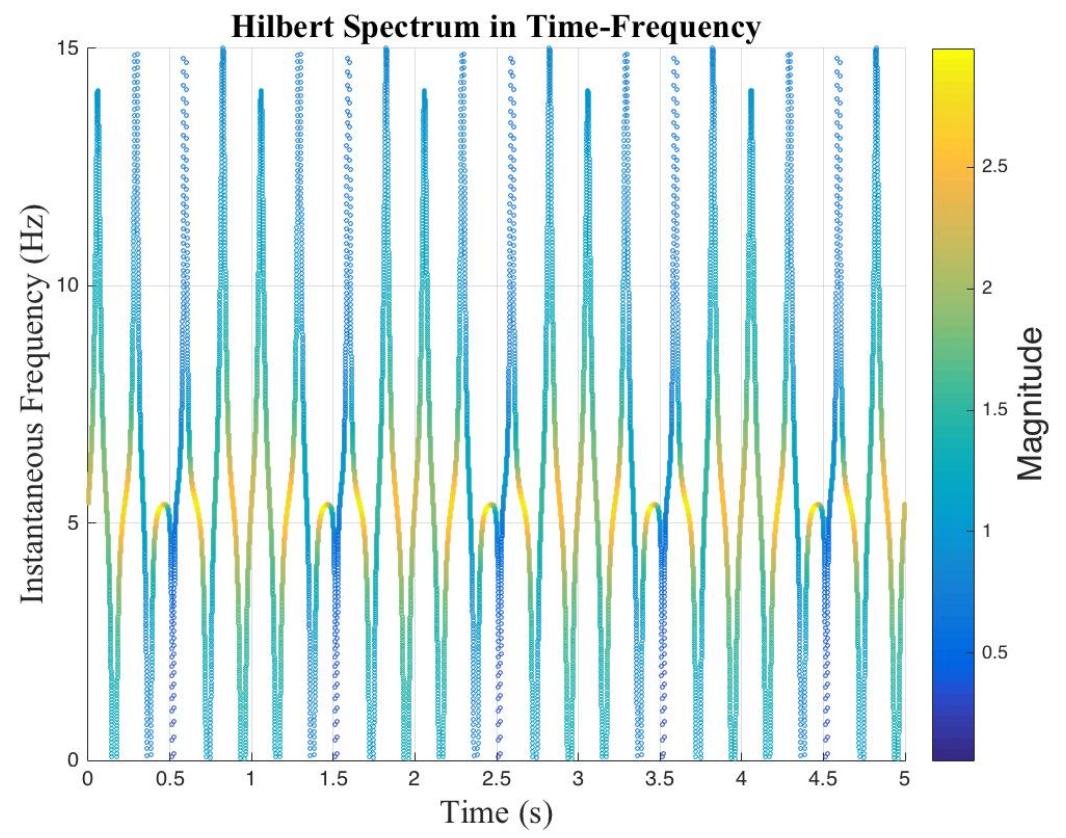

Figure 1.28. The Hilbert spectrum of the multi-component signal 

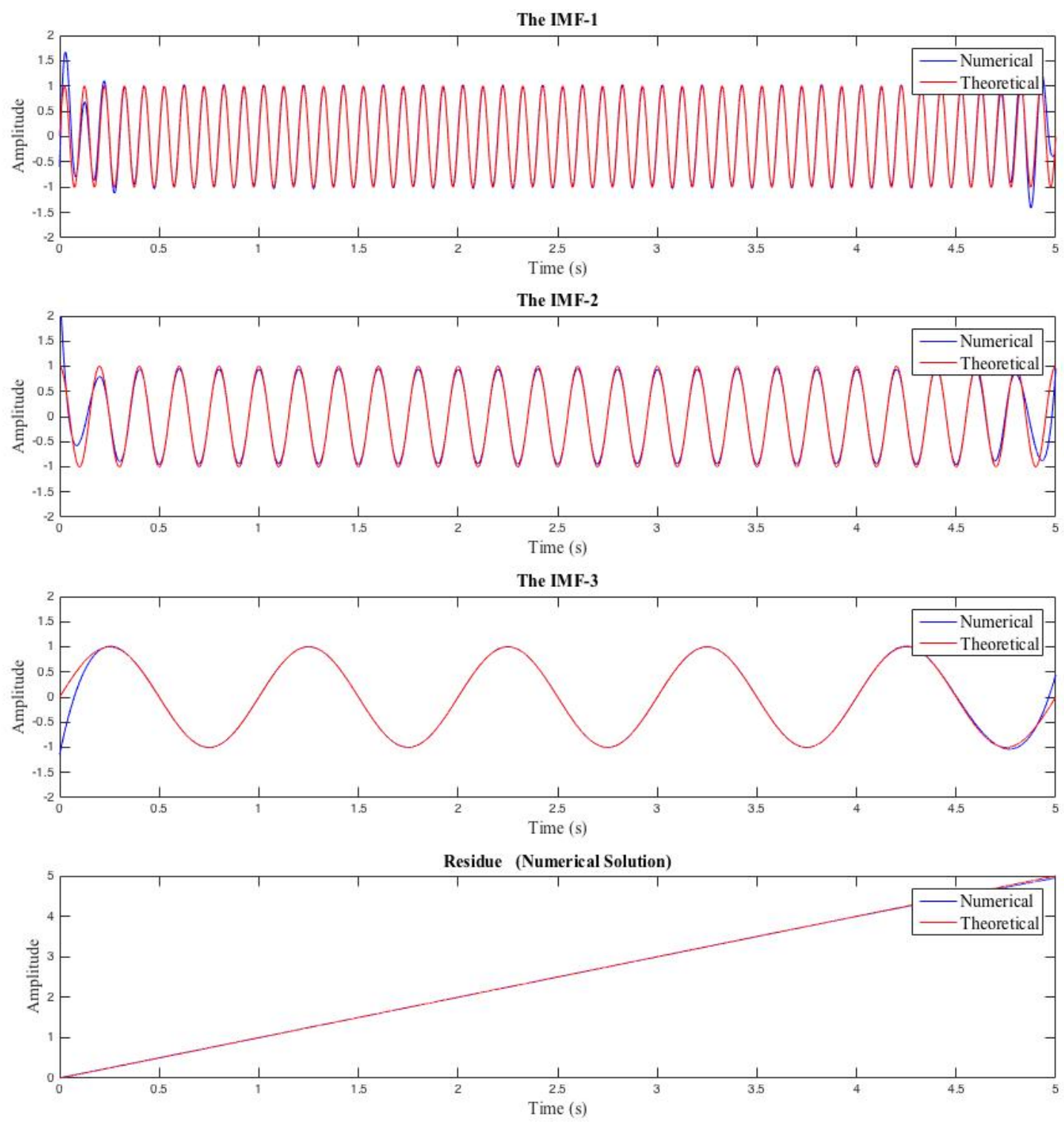

Figure 1.29. The numerical (the blue curve) and theoretical (the red curve) IMFs,

And residue of the multi-component signal 


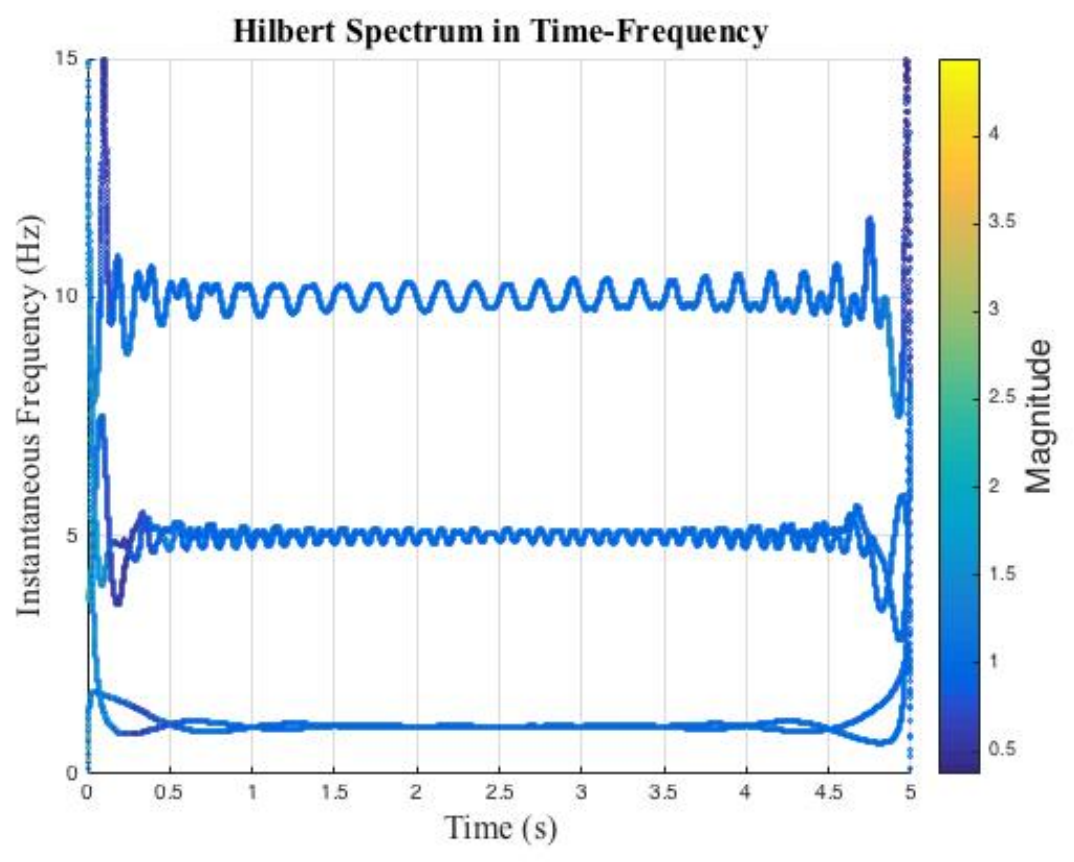

Figure 1.30. The numerical HHT spectrum

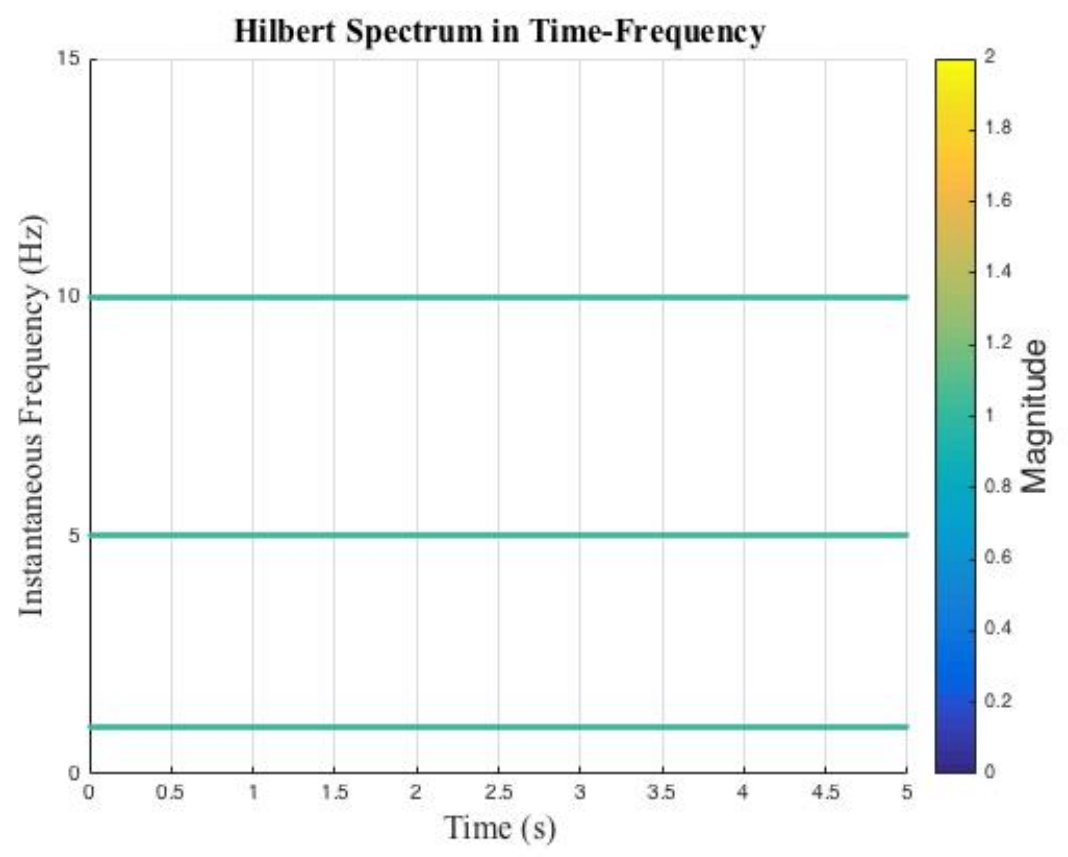

Figure 1.31. The theoretical HHT spectrum 


\subsubsection{Limitations and Comparison of HHT}

\section{1) End effect}

Hilbert Huang transform (HHT) consists of Empirical Mode Decomposition (EMD) and Hilbert Spectral Analysis (HSA), but there is an unwanted end effect exist in the EMD process. In EMD, the end effect happens at each end of the signal, since the end point typically is not the extreme point, but it is usually considered as one for spline interpolation when generating the envelope of maximum or minimum. The error occurs at the end of the signal, and is accumulated during iteration and sifting process. The end effect can be seen obviously in the Figure 1.30. There are some errors shown at the end of signals by comparing the numerical result and the theoretical result [2.15].

The end effect differs as the different sampling points, sampling length or algorithm of generating envelopes. There are some end extending methods and alternative interpolation methods are developed for eliminating end effect in the EMD [2.19][2.20].

\section{2) Adjacent frequencies}

Even though EMD is an effective tool for separating each IMF with different oscillation frequencies, it has limitation in dealing with signals contains IMFs with adjacent frequencies. Some analytically distinguishable adjacent IMFs are not significantly different for numerical methods. To be quantitative, for frequencies different by at least 5 times, EMD can yield acceptable result without the problem of adjacency; 10 times is desirable for good result [2.15]. 


\section{3) Mode Mixing}

Mode mixing is a problem of mode rectification for IMFs, which happens during the sifting process in EMD. Straightforward implementation of the sifting procedure yields the mode mixing problem, further leading to aliasing in the IMFs. Mode mixing problem make the IMF lose its physical meaning and lack of physical uniqueness [2.21], which means IMFs can be so different due to adjustable parameters in sifting process. Huang et al. first invoked an intermittence test for eliminating the mode mixing problem [2.8]. But the technique can only be applied through interactive steps. And then Ensemble Empirical Mode Decomposition (EEMD) was introduced in 2009, which significantly solved the mode mixing problem by adding white noise distribution evenly onto the raw signal [2.4]. Recently, a new method named Phase Space Ensemble Empirical Mode Decomposition (PSEEMD) integrating Phase Space Reconstruction (PSR) and Manifold Learning (ML) was proposed for modifying EEMD for better efficiency and convenience [2.22].

Even though HHT does have its problems, it possesses great potential for solving non-linear or non-stationary problems. The comparison between HT/HHT and other popular tools, non-linear or non-stationary signals, in general, for time-frequency analysis is given here.

\section{1) Comparison between HHT and STFT}

For stationary data analysis, Fourier spectrum can provide meaningful information about frequencies, amplitudes and energy distributions with Fourier transform. But for non-stationary data, ex: earthquake, since Fourier transform can only yields average characteristics globally, it lose the locality of needed information. STFT is developed for dealing with non-stationary data by 
adding window to extract the data locally. However, for better locality of the analysis, the window needs to be shorter while sacrificing the resolution in frequency [2.6][2.23]. Compared to STFT, HHT provide much higher resolution and sharper identification of energy distribution in timefrequency domain, due to its instantaneous frequency characteristic, providing great applicability in nonstationary time-frequency analysis [2.6].

\section{2) Comparison between HHT and WT}

WT, known as most widely used, has become one of the most fast-mathematical and signal processing technique for time-frequency analysis. Being complete, orthogonal, local and adaptive, it is suitable for dealing with non-linear and non-stationary signals and obtaining both time and frequency information simultaneously. However, it has certain inevitable deficiencies which make the results confusing and less interpretable, including interference terms, border distortion and energy leakage [2.7][2.24].Even though WT is good at processing nonstationary signal, it cannot provide high resolution in both time and frequency at the same time because of the limitation of Heisenberg-Gabor inequality [2.22]. HHT not only has better resolution, compared to WT, both in time and frequency but also better computational efficiency.

\subsection{Riesz Transform and Monogenic Signal}

The complex-valued representation of any real signal, which is also named as the analytic signal representation, is commonly used as an alternative representation for simple mathematical representation and computational efficiency; however, every real signal requires an appropriately 
derived imaginary part to construct the complex representation without loss of signal energy and phase distortion. Hilbert transform (HT) is a well-known tool to compute the imaginary parts of real-valued signals in order to generate its analytic signal. HT provides local amplitude and phase information through generating analytic signal along any orientation of interest. HT is defined as the convolution of $1 / \pi t$ and the real function $f(t)$, which in frequency domain is the product of $i \operatorname{sgn} \omega$ and Fourier transformed function $\tilde{f}(\omega)$, as shown in Eq.(2.90).

$$
\mathcal{H}[f(t)]=\frac{1}{\pi t} \otimes f(t) \stackrel{F F T}{\longrightarrow} i \operatorname{sgn} \omega \cdot \tilde{f}(\omega)
$$

where $\operatorname{sgn}(\omega)=\left\{\begin{array}{cl}1 & , \text { for } \omega>0 \\ 0 & , \text { for } \omega=0 \\ -1 & , \text { for } \omega<0\end{array}, \mathcal{H}(\cdot)\right.$ is Hilbert transform operator, $\otimes$ denotes convolution operator, and $\tilde{f}(\omega)$ is the fast Fourier transform (FFT) of the function $f(t)$. The HT of the $f(t)$ is the purely imaginary part $f_{H}(t)$ of the analytic signal $f_{+}(t)$, where $f_{+}(t)=f(t)+i f_{H}(t)$, and $f_{H}(t)$ is also called the quadrature pair of the real signal $f(t)$. The analytic signal $f_{+}(t)$ can be expressed in a polar form with amplitude $A(t)$ and phase $\phi(t)$ as shown in Eq.(2.91), where $A(t)=\sqrt{|f(t)|^{2}+\left|f_{H}(t)\right|^{2}}$, $\phi(t)=\tan ^{-1}\left[f_{H}(t) / f(t)\right]$. The instantaneous frequency $\omega(t)$ can be further calculated as the derivative of the instantaneous phase $\phi(t)$ as the expression $\omega(t)=d \phi(t) / d t$.

$$
f_{+}(t) \equiv A(t) e^{i \phi(t)}
$$

HT has been considered as a robust time-frequency analysis tool, and it can be further employed for space-wavenumber analysis when HT is operated in space domain to obtain the local instantaneous spatial phase and local instantaneous wavenumber (IW). A damage imaging 
condition using IW was developed and applied to highlight the local wavenumber induced by the discontinuity of material properties around the damage area. Since HT is only capable of dealing with one-dimensional (1-D) signal, the IW was calculated by employing HT and taking derivative of the instantaneous spatial phase with respect to two orientations separately [2.33]. However, the generalization of two-dimensional (2-D) IW obtained from the spatial phase by simply calculating HT twice at each orientation is not isotropic and lacks the invariance-equivariance property for local phase and amplitude [2.27].

Given a displacement function of the plane wavefield $w(x, y, t)$ at any time instant $t$, it can be represented as the real part of the complex-valued signal by defining the phase of the signal $\phi(x, y, t)=k_{x} x+k_{y} y-\omega t=\mathbf{k x}-\omega t$, where $k_{x}, k_{y}$ is the wavenumber along $x, y$ direction respectively, $\mathbf{k}=\left[k_{x}, k_{y}\right]^{\prime}$ is the direction of wave propagation and $\omega$ is the temporal frequency as shown below.

$$
w(x, y, t)=\operatorname{Re}\left[e^{i\left(k_{x} x+k_{y} y-\omega t\right)}\right]=\cos \left(k_{x} x+k_{y} y-\omega t\right)=\cos \phi
$$

At a given time instant $t$, the instantaneous wavefield can be considered as an image snapshot. Since the wavefield is 2-D signal, two imaginary parts are needed to construct the quadrature image for obtaining the complex-valued wavefield. Therefore, Riesz transform (RT) and the monogenic signal of Felsberg and Sommer [2.28], proven to be appropriate 2-D generalization of HT and analytic signal, are introduced to derive the instantaneous wavenumber of the wavefield. For the displacement $w(x, y, t)$ at a given time instant $t$, the definition of RT is given by

$$
\mathcal{R}[w(x, y, t)]=-\frac{\mathbf{x}}{2 \pi|\mathbf{x}|^{3}} \otimes w(x, y, t) \stackrel{2-\mathrm{DFFT}}{\longrightarrow} i \frac{\mathbf{k}}{|\mathbf{k}|} \tilde{w}\left(k_{x}, k_{y}, t\right)
$$


where $\mathcal{R}(\cdot)$ denotes RT operator, $\otimes$ denotes convolution operator, $\tilde{w}\left(k_{x}, k_{y}, t\right)$ is the spatially 2-D FFT of the wavefield at time $t, \mathbf{x}=[x, y]^{T}$ is the local spatial coordinate, and $\mathbf{k}=\left[k_{x}, k_{y}\right]^{T}$ is the local coordinate in wavenumber domain.

Similarly, the RT can be considered as a product of the spatially Fourier transformed signal and the RT transfer function in the wavenumber domain. The two imaginary parts for constructing the quadrature image are then:

$$
\begin{aligned}
& \tilde{\hat{w}}_{|x|}\left(k_{x}, k_{y}, t\right)=\left\{\begin{array}{lr}
i \frac{k_{x}}{|\mathbf{k}|} \tilde{w}\left(k_{x}, k_{y}, t\right), & \mathbf{k} \neq 0 \\
0, & \mathbf{k}=0
\end{array}\right. \\
& \tilde{\hat{w}}_{|y|}\left(k_{x}, k_{y}, t\right)=\left\{\begin{array}{lr}
i \frac{k_{y}}{|\mathbf{k}|} \tilde{w}\left(k_{x}, k_{y}, t\right), & \mathbf{k} \neq 0 \\
0, & \mathbf{k}=0
\end{array}\right.
\end{aligned}
$$

where $\tilde{\hat{w}}_{|x|}\left(k_{x}, k_{y}, t\right)$ and $\tilde{\hat{w}}_{|y|}\left(k_{x}, k_{y}, t\right)$ are the RT of the real wavefield along $x$-axis and $y$-axis in wavenumber domain, and the two imaginary parts to construct the quadrature image and the monogenic wavefield are the inverse FFT of $\tilde{\hat{w}}_{|x|}\left(k_{x}, k_{y}, t\right)$ and $\tilde{\hat{w}}_{|x|}\left(k_{x}, k_{y}, t\right)$. The RT operator $i \frac{k_{x}}{|\mathbf{k}|}$ and $i \frac{k_{y}}{|\mathbf{k}|}$ in wavenumber domain is illustrated in Figure 1.32(a) and Figure 1.32(b) respectively. 


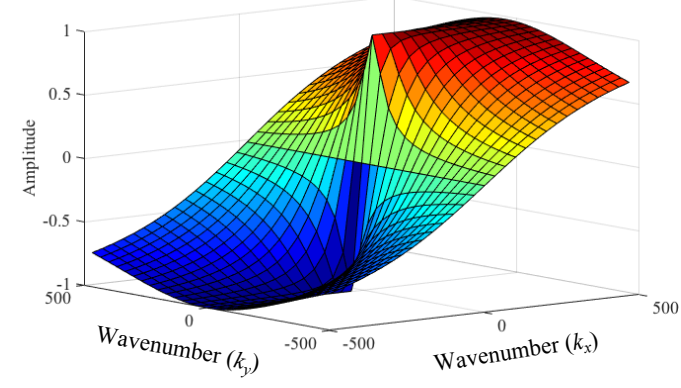

(a)

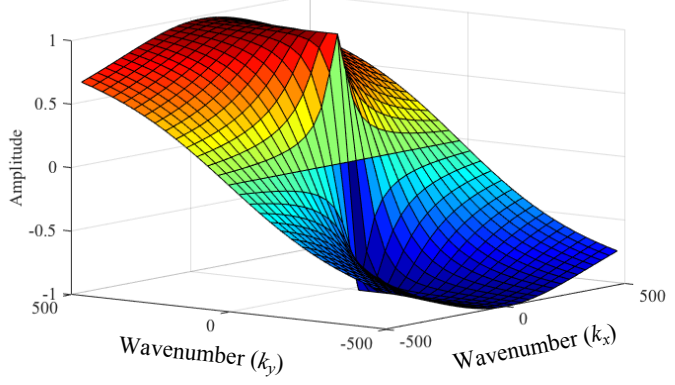

(b)

Figure 1.32. Illustrastion of Riesz transform operator in wavenumber domain along (a) $x$-axis as shown in Eq.(1.121), (b) $y$-axis as shown in Eq.(1.122).

The Riesz transformed wavefield signal can be represented as a Riesz triple vector [2.27] for the construction of monogenic signal as:

$$
w_{+}(x, y, t)=\left[w(x, y, t), \hat{w}_{|x|}(x, y, t), \hat{w}_{|y|}(x, y, t)\right]^{T}
$$

where $w_{+}(x, y, t)$ is the monogenic signal of the original wavefield, and $\hat{w}_{|x|}, \hat{w}_{|y|}$ are the RT along $x$ and $y$ axis respectively. Also, from the monogenic signal, the instantaneous wave energy is defined as

$$
\mathrm{E}(x, y, t)=|w(x, y, t)|^{2}+\left|\hat{w}_{|x|}(x, y, t)\right|^{2}+\left|\hat{w}_{|y|}(x, y, t)\right|^{2}
$$

And the local instantaneous amplitude which is also the envelope of the monogenic signal, is defined as 


$$
\mathrm{A}(x, y, t)=\sqrt{|w(x, y, t)|^{2}+\left|w_{R}(x, y, t)\right|^{2}}
$$

where $w_{R}(x, y, t)=\sqrt{\left|\hat{w}_{|x|}(x, y, t)\right|^{2}+\left|\hat{w}_{|y|}(x, y, t)\right|^{2}}$ is the RT steered to the dominant orientation. The spatial phase $\phi(x, y, t)$ and the Riesz orientation $\theta(x, y, t)$ at any instant can be calculated from the Riesz triple vector as shown in Eq. (2.99) and (2.100), and the illustration for the concept of orientation, phase direction and the wavefront is shown in Figure 1.33.

$$
\begin{aligned}
& \phi(x, y, t)=\tan ^{-1}\left(\frac{w_{R}(x, y, t)}{w(x, y, t)}\right) \\
& \theta(x, y, t)=\tan ^{-1}\left(\frac{\hat{w}_{|y|}(x, y, t)}{\hat{w}_{|x|}(x, y, t)}\right)
\end{aligned}
$$

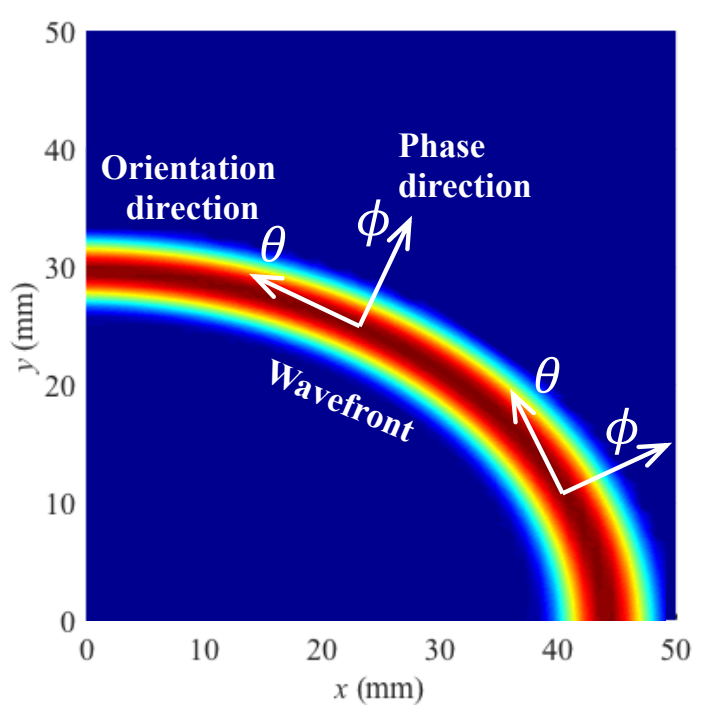

Figure 1.33. The illustration of wavefront, orientation and dominant phase direction as described in Eq. (1.126) and (1.127). 
The spatial phase here is defined as the phase derived along the dominant direction normal to the wavefront, where the wavefield signals possess the maximum variation, which is the direction of phase velocity; the Riesz orientation is defined tangent to the wavefront, where the wave signal is least changing [2.28]. In short, at any given $x, y$ and $t$, the Riesz representation can be written in terms of the local amplitude $A$, the local dominant orientation $\theta$ and the local spatial phase $\phi$ as [2.29]:

$$
w=\mathrm{A} \cos \phi, \hat{w}_{|x|}=\mathrm{A} \sin \phi \cos \theta, \hat{w}_{|y|}=\mathrm{A} \sin \phi \sin \theta
$$

and

$$
w=\mathrm{A} \cos \phi, w_{R}=\mathrm{A} \sin \phi
$$

The RT steered to its dominant orientation is the quadrature pair of the original wavefield signal at the given location and instant, and it can be applied as the imaginary part to generate the analytic signal as Eq.(1.124). Therefore, the steered RT can be considered as the HT along its dominant orientation.

$$
w_{+}=w+i w_{R}=\mathrm{A} \cos \phi+i \mathrm{~A} \sin \phi=\mathrm{A} e^{i \phi}
$$

The local spatial phase derived from RT is exactly the phase of the original wavefield $\phi(\mathbf{x}, t)=\mathbf{k x}-\omega t$. The instantaneous wavenumber via Reisz transform (IW-R) is then defined as Eq. (3.15), which is the instantaneous wave vector.

$$
\mathbf{k}=\nabla \phi(\mathbf{x}, t)=\left[\frac{\partial \phi}{\partial x}, \frac{\partial \phi}{\partial y}\right]^{\prime}
$$


However, the phase derived directly from the aforementioned calculation with Riesz triple vector sometimes can encounter the problem of phase ambiguity. Since the definition of the local phase depends on the orientation, so that the phase and the orientation is variant between $(\phi, \theta)$ and $(-$ $\phi, \theta+\pi)$ and it leads to the ambiguity of the phase. The quaternionic representation of RT was previously introduced by Neal et al. to obtain phase information of $\phi \sin \theta$ and $\phi \cos \theta$ which are invariant to the sign ambiguity in direct RT [2.30]. With the pre-defined quaternionic phase, the quaternionic representation of the Riesz transformed wavefield can be written as:

$$
\mathbf{Q}=w+i \hat{w}_{|x|}+j \hat{w}_{|y|}
$$

Replacing the parameters with amplitude, phase and orientation, the quaternion form becomes:

$$
\mathbf{Q}=\mathrm{A} \cos \phi+i \mathrm{~A} \sin \phi \cos \theta+j \mathrm{~A} \sin \phi \sin \theta
$$

The quaternionic phase can then be derived as the natural log of the normalized quaternion instead of solving regular RT formulation as:

$$
\log \left(\frac{\mathbf{Q}}{\|\mathbf{Q}\|}\right)=\log (\cos \phi+i \sin \phi \cos \theta+j \sin \phi \sin \theta)=i \phi \cos \theta+j \phi \sin \theta
$$

The phase without ambiguity can be further computed with two coefficients of imaginary part in quaternionic representation of RT as:

$$
\phi=\cos \theta(\phi \cos \theta)+\sin \theta(\phi \sin \theta)
$$

Then the instantaneous spatial phase derived by Riesz transform can be further taken derivative in order to obtain the instantaneous wave vector (spatial frequency). 


\subsection{Applications}

Due to high time-frequency resolution and good computational efficiency, Hilbert Transform/Hilbert-Huang Transform (HT/HHT) provides a powerful solution for solving nonlinear and non-stationary problems in the signal processing of strongly transient time-series signals. Thus HT/HHT is now comprehensively applied in the field of Structural Health Monitoring, Fault Diagnosis and Geophysical Studies, etc.; moreover, after being extended from 1-D to 2-D, the HT/HHT is now also popular for Image Processing, Ocean Studies, etc. This section mainly focuses on either current application or HT/HHT as a prospective tool or technique for SHM due to its excellent potential in the application of SHM.

\section{Application of HT/HHT for wave-based structural health monitoring}

In SHM, the analysis of the wave propagating in the structure is nearly the most important and fundamental technique for further damage visualization and characterization. Abundant valuable information is concealed in the wave obtained by either Laser Doppler Vibrometer (LDV) or array of two or more piezoelectric sensors; therefore, effective and efficient signal processing methods are necessary to extract the information needed for imaging methods, such as zero-lag crosscorrelation (ZLCC) [2.25], reverse-time migration (RTM) [2.31], cumulative standing wave energy (CSWE) [2.32] and instantaneous wavenumber (IW) [2.33], etc. 

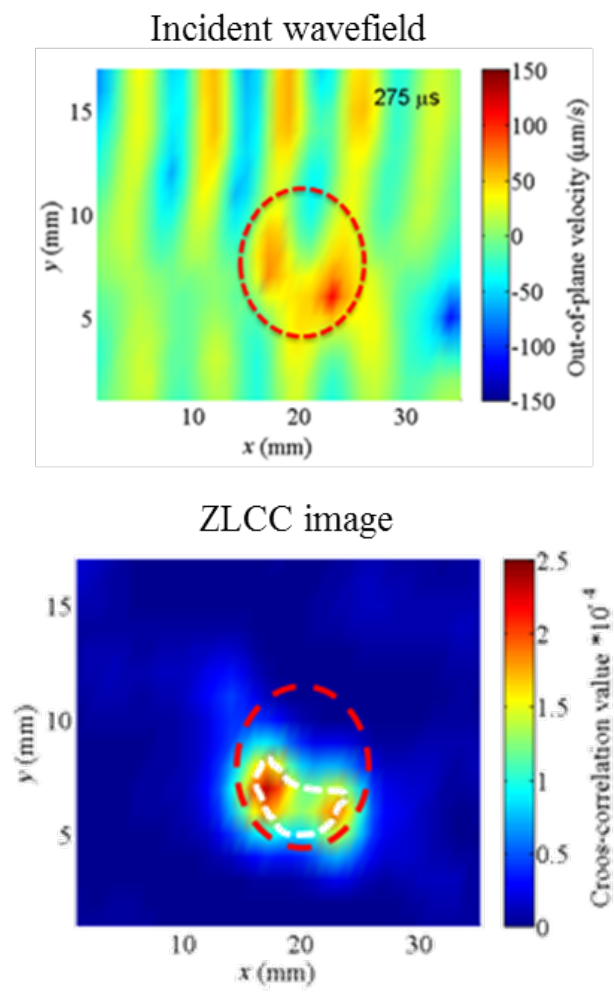

Reflected wavefield

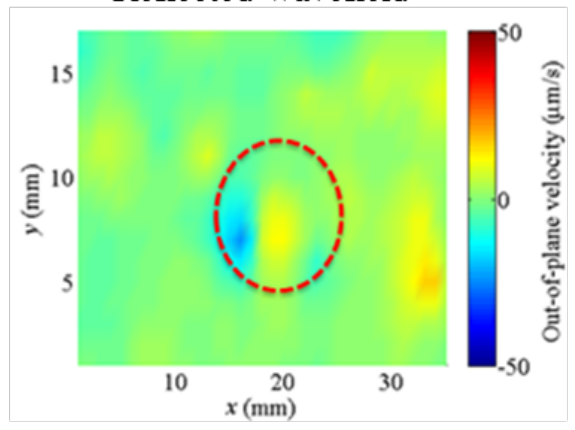

C-scan image

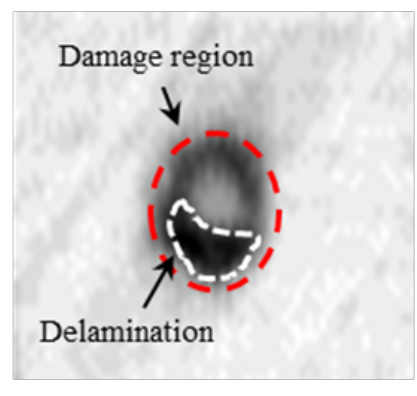

Figure 1.34. The wave filtering and ZLCC imaging method

Currently by separating incident wave, reflective wave and standing wave in frequencywavenumber domain with wave-filtering technique, the image of damage location and contour can be shown with further imaging methods, e.g., ZLCC, CSWE, as shown in Figure 1.34. For instance, in ZLCC method, the whole wavefield measured is separated to incident wave and reflected wave, caused by the damage, in frequency-wavenumber domain after 3-D Fourier transform, in regard to the positive and negative wavenumber, which represent two opposite propagating directions. The damage can then be localized and identified by cross-correlating two waves with zero timelag [2.25]. ZLCC is now well-developed as a robust tool for damage visualization and characterization; however, the imaging quality and accuracy highly depends on the wave separation technique. 
Since the efficiency and the effectiveness of the wave filtering are relevant to the quality and the precision of the damage localization, HT/HHT is potentially much more a powerful tool in wave filtering. Either HT or EMD can be generalized or extended to 2-D spatial analysis tool. The generalized Hilbert transform, also named Riesz transform [2.26]is one of the image processing techniques that can be potentially applied for separating incident/reflected waves exactly along its dominant direction locally at each point, yielding more accurate wave separation than traditional wave filtering in fixed global coordinate [2.27].

HT/HHT can not only be a new effective wave filtering tool with high resolution, but also be used to extract more physical meaningful instantaneous parameters for enhancing the imaging methods. By applying HT in spatial domain, the time-frequency analysis becomes the space-wavenumber analysis, so that instantaneous wavenumber, physically known as spatial frequency, is obtained as a function of any location and instant of time. Since the wavenumber changes as effective thickness changes during wave propagation on a plate-like structure, the IW contour map of the wavefield can be translated to an effective thickness map to indicate the thickness change as damage area, as shown in Figure 1.35, which is physically similar to the effect of delamination damage for composite structure [2.33].

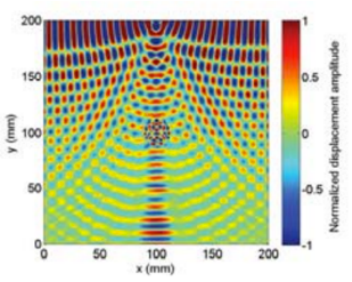

(a)

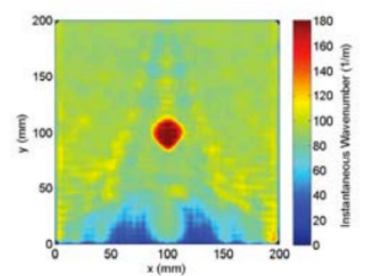

(b)

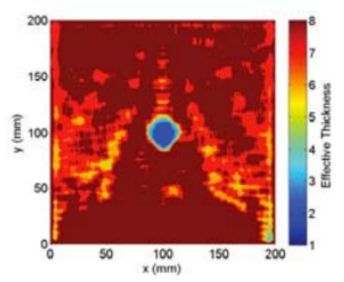

(c)

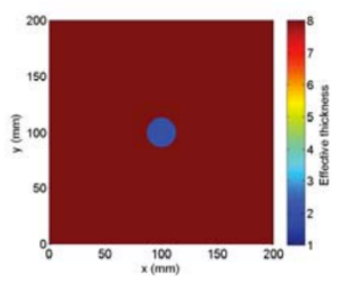

(d)

Figure 1.35. (a) Frequency-space domain, (b) Instantaneous wavenumber map, (c) Effective thickness map, and (d) Theoretical effective thickness map [2.33].. 
The standing wave trapped on the surface of the delamination for composite structure is one of important phenomenon for the damage detection. After being isolated from the entire wavefield, the standing wave on the damage area can reveal not only the damage area but also even more detailed information with further analysis of parameters derived [2.34], e.g. frequency, mode shape, etc. HT/HHT will potentially become a new practical method for the real-time identification of the traveling waves [2.35] and damage visualization [2.36]. The 2-D EMD can be applied to wavefield image processing for spatial decomposition, and the HAS can be further used for analyzing instantaneous parameters to get better damage characterization [2.37][2.38].

HT/HHT now has been applied to analyzing dispersion of seismic surface-waves. Based on the concepts of instantaneous amplitude and instantaneous phase, the dispersion of both group and phase velocities could be obtained from the derived time-frequency spectrum with good agreements with the theoretical values [2.6]. Besides, the analytical representation using HT/HHT is not only helpful to get the envelope of a monochromatic internal wave in fluids, but also effective to quantify the dissipation behavior. For measuring the dissipation effect, its complex analytical representation of the wavefield signal is taken to extract the envelope and the phase of the waves [2.39], further discriminating the different internal waves at any specific frequency. These applications provide sharp and accurate time-frequency analysis results, showing an encouraging capability for the analysis of dispersive and dissipative elative-waves propagating in plates as an effective and accurate technique for SHM. 


\section{Application of HT/HHT for system identification in structural health monitoring}

For the system identification in SHM, besides extracting the time-frequency information, the $\mathrm{HT} / \mathrm{HHT}$ is also applied for vibration modal analysis to identify parameters of a vibrating structure. As an adaptive without prior assumption signal processing technique, HT/HHT has been comprehensively applied in the analysis of structural modal responses [2.38]. With the introduction of HT/HHT to the system identification of SHM, parametric identification as well as further interpretation of nonlinear and nonstationary mechanical vibrating system has become more robust, reliable and convincing [2.41].

If there is any damage happens to a structure, physical properties of the structure changes leading to the changes in modal properties. So that analysis of modal behavior can be used for damage detection. Traditional WT can successfully identify the damage time instant and damage location [2.42][2.43], but with noise pollution, the identification of damage spikes based on WT involves lots of uncertainties.

A novel method based on EMD with random decrement technique (RDT) was proposed to detect the damage existence and location [2.44][2.45]. Simulation results demonstrated that the proposed method was capable of detecting the damage time instant, identifying the system parameters and eventually determining the existence and location of damage by detecting the gradual change of natural frequencies in time domain because of the instantaneous frequency of HHT. However, for localizing damage, the response time histories of all DOF are necessary. 


\section{Application of HT/HHT for vision-based structural health monitoring}

An advanced video magnification techniques were proposed recently with great potential in structural health monitoring. The technique allows the subtle motion invisible to naked eyes become inviable by amplifying the wanted motion in specific region and given frequencies [2.46]. The technique needs only a standard video as input file, by applying temporal and spatial filters, a video can be decomposed into pixels for each frame. The subtle motion can be extracted and magnified to reconstruct the video revealing hidden information.

Eulerian motion magnification method was first developed mathematically based on first-order linear Taylor- series expansion in 2012 [2.46],which was fast and simple, but limited in scaling factor and sensitive to noise characteristic. To avoid the noise from being magnified, complex steerable pyramids phase-based motion magnification was then developed [2.47] for increasing scaling factor and signal-to-noise (SNR) behavior. The complex steerable pyramids phase-based method is a fantastic application of Hilbert transform. In the given orientation, after the Fourier decomposition in spatial domain and a temporal filter in time domain, the HT is applied to each sub-band for obtaining phase change, which represents the pre-amplified displacement. Multiplying a magnifying factor for increasing the phase of the sub-band, the targeted displacement is magnified without amplifying noise simultaneously. However, it was computationally costly due to the over-completeness caused by multi-orientation. Riesz-pyramid was brought up to replace the complex steerable pyramids for the computational efficiency while keeping similar image quality. The Reisz-pyramid fast phase-based method is introduced for increasing the computational efficiency by substituting the user-defined global multiple orientations with local dominant orientation computed by Reisz transform [2.26]. 
The complex steerable pyramid phase-based motion magnification was used to derive the modal shape of a cantilever beam [2.49]. The displacement signal was first extracted from the video recorded at 2000 frames per second and then applied with fast Fourier Transform. Peaks in the Fourier transform were picked as modal frequencies. The modal shapes were further extracted by using motion magnification method at the previously determined modal frequencies. The results from cameras were compared with results from laser vibrometer and accelerometers. Even though only the first four resonant frequencies could be extracted by camera, and the noise floor of the signal was relatively high compared to the other two methods, the high spatial resolution provided by camera showed the potential of the application in the field of structural health monitoring.

In order to achieve real time analysis for modal shape, Riesz pyramid phase-based motion magnification method was used to repeat the cantilever beam experiment[2.49]. The processing speed increase to 35 frames per second, and the second and third mode can be shown in real-time. Due to the previously mentioned noise problem, the frequency domain decomposition (FDD) method was applied to identify the resonant modal frequencies, which had better performance than peak-picking method especially in the case that signal was noisy.

The material properties were also measured by analyzing the small amplitude vibration with motion magnification method [2.50]. The Young's modulus for a rod with known geometry was derived from the relationship between natural frequency, geometry, density and stiffness. The resonant frequencies can be known by applying the peak-picking method in the Fourier transform of the motion captured by camera.

The application in the field of structural health monitoring is currently limited to the resolution, the frame rate and the exposure time of camera, and the processing speed of computer. The modal 
shape or operational deflection shape (ODS) is considered for the application for motion magnification in structural health monitoring, since it is possible to be captured with current high speed camera with limited frame rate. However, the kernel of the motion magnification, the concept Hilbert transform, is not only limited to magnifying motion of the video. It could be extracted and further applied onto imaging the damage in the structure based on wave propagation. 


\subsection{References}

[2.1] M. Johansson, "The Hilbert Transform, Master Thesis in Mathematics/Applied Mathematics," Växjö University, 1999.

[2.2] M. Feldman, "Vibration Analysis of Non-Symmetric Elastic Force Systems via The Hilbert Transform," Proceedings of SPIE, the International Society for Optical Engineering, vol. 3089, pp.1017-1022, 1997.

[2.3] M. Feldman, "Hilbert Transform in Vibration Analysis," Mechanical Systems and Signal Processing, vol.25, no.3, pp.735-802, 2011.

[2.4] Z. Wu, and N.E. Huang, "Ensemble Empirical Mode Decomposition: A Noise-Assisted Data Analysis Method," Advances in Adaptive Data Analysis, vol.1, no.1, pp.1-41, 2009.

[2.5] M. Dätig, and T. Schlurmann, "Performance and Limitations of The Hilbert-Huang Transformation (HHT) with an Application to Irregular Water Waves," Ocean Engineering, vol.31, no.14, pp.1783-1834, 2004.

[2.6] C.H. Chen, C.P. Li, and T.L. Teng, "Surface-wave Dispersion Measurements Using HilbertHuang Transform," Terrestrial, Atmospheric and Oceanic Sciences (TAO), vol.13, no.2, pp.171-184, 2002.

[2.7] Z.K. Peng, P.W. Tse, and F.L. Chu, "A comparison Study of Improved Hilbert-Huang Transform and Wavelet Transform: Application to Fault Diagnosis for Rolling Bearing,” Mechanical Systems and Signal Processing, vol.19, no. 5, pp.971-988, 2004.

[2.8] N.E. Huang, Hilbert-Huang Transform and Its Applications, pp.1-26, 2005.

[2.9] A.D. Poularikas, "The Hilbert Transform, The Handbook of Formulas and Tables for Signal Processing," The Handbook of formulas and Tables for Signal Processing, 1999.

[2.10] F.R. Kschischang, “The Hilbert Transform,” University of Toronto, 2006. 
[2.11] S.L. Hahn, "Hilbert Transforms,” The Transforms and Applications Handbook, pp.463-629, 1999.

[2.12] A. Papoulis, The Fourier integral and its applications, McGraw-Hill, 1962.

[2.13] B. Liu, S. Riemenschneider, and Y. Xu, "Gearbox Fault Diagnosis Using Empirical Model Decomposition and Hilbert Spectrum," Mechanical Systems and Signal Processing, vol.20, pp.718 -734. 2006.

[2.14] P. D. McFadden, "Detecting Fatigue Cracks in Gears by Amplitude and Phase Modulation of the Meshing Vibration," Journal of Vibration Acoustics Stress and Reliability in Design, vol.108, no.2, pp.165-170, 1986.

[2.15] M. Klingspor, "Hilbert Transform: Mathematical Theory and Applications to Signal Processing," 2015.

[2.16] E. Bedrosian, “A Product Theorem for Hilbert Transform,” Proceedings of the IEEE, vol.51, no.5, pp.868-869, 1963.

[2.17] P. J. Schreier, and L.L. Scharf, "Statistical Signal Processing of Complex-Valued Data," Cambridge: Cambridge University Press, 2010.

[2.18] N. E. Huang, et al., "The Empirical Mode Decomposition and The Hilbert Spectrum for Nonlinear and Non-stationary Time Series Analysis." Proceedings of the Royal Society of London A: Mathematical, Physical and Engineering Sciences, vol.454, no.1971, 1998.

[2.19] Y. Guang, X. Sun, M. Zhang, X. Li, and X. Liu, "Study on Ways to Restrain End Effect of Hilbert-Huang Transform," Journal of Computers, vol.25, 2014.

[2.20] R.T. Rato, M.D. Ortigueira, and A.G. Batista, "On the HHT, its problems, and some solutions," Mechanical Systems and Signal Processing, vol.22, no.6, pp.1374-1394, 2008. 
[2.21] N.E. Huang, and Z. Wu, "A Review on Hilbert-Huang Transform: Method and its Applications to Geophysical Studies," Review of Geophysics, vol.46, no.2, 2008.

[2.22] Z. Ma, G. Wen, and C. Jiang, "EEMD Independent Extraction for Mixing Features of Rotating Machinery Reconstructed in Phase Space," Sensors, vol.15, no.4, pp.8550-8569, 2015.

[2.23] R.R. Zhang, S. Ma, E. Safak, and S. Hartzell, "Hilbert-Huang Transform Analysis of Dynamic and Earthquake Motion Recordings," Journal of Engineering Mechanics, vol.129, no.8, pp.861-875, 2003.

[2.24] Z.K. Peng, P.W. Tse, and F.L. Chu, "An Improved Hilbert-Huang Transform and Its Application in Vibration Signal Analysis," Journal of Sound and Vibration, vol.286, no.12, pp.187-205, 2005.

[2.25] M.S. Harb, and F.G. Yuan, "Barely Visible Impact Damage Imaging Using Non-Contact Air-Coupled Transducer/Laser Doppler Vibrometer System,” Structural Health Monitoring, 2016.

[2.26] N. Wadhwa, M. Rubinstein, F. Durand, and W.T. Freeman, "Riesz Pyramids for Fast Phasebased Video Magnification,” Computational Photography (ICCP), 2014 IEEE International Conference on. IEEE, pp.1-10, 2014.

[2.27] K. Langley, and S.J. Anderson, "The Riesz Transform and Simultaneous Representations of Phase, Energy and Orientation in Spatial Vision," Vision Research, vol.50, no.7, pp.1748$1765,2010$.

[2.28] M. Felsberg and G. Sommer," The monogenic signal," IEEE Transactions on Signal Processing, vol.49, no.12, pp.3136-3144, 2001. 
[2.29] N. Wadhwa, M. Rubinstein, F. Durand and W.T. Freeman, "Riesz pyramids for fast phasebased video magnification," In Computational Photography (ICCP), 2014 IEEE International Conference on , pp.1-10, 2014.

[2.30] N. Wadhwa, M. Rubinstein, F. Durand, F. and W.T. Freeman, "Quaternionic representation of the riesz pyramid for video magnification,” 2014.

[2.31] J. He, and F.G. Yuan,” A Quantitative Damage Imaging Technique Based on Enhanced CCRTM for Composite Plates Using 2D Scan,” Smart Materials and Structures, vol.25, no.10, 2016.

[2.32] Y.K. An, B. Park, and H. Sohn," Complete Noncontact Laser Ultrasonic Imaging for Automated Crack Visualization in a Plate," Smart Materials and Structures, vol.22, no.2, 2013.

[2.33] O. Mesnil, C.A.C. Leckey, and M. Ruzzene, "Instantaneous and Local Wavenumber Estimations for Damage Quantification in Composites," Structural Health Monitoring, 2014.

[2.34] H. Sohn, et al., " Delamination Detection in Composites Through Guided Wave Field Image Processing," Composites Science and Technology, vol.71, no.9, pp.1250-1256, 2011.

[2.35] A. Minikes, et al., "On The Sensing and Tuning of Progressive Structural Vibration Waves," IEEE Transactions on Ultrasonics, Ferroelectrics, and Frequency Control, vol.52, no.9, pp.1565-1576, 2005.

[2.36] M. Ruzzene, "Frequency-Wavenumber Domain Filtering for Improved Damage Visualization," Smart Materials and Structures, vol.16, pp.2116-2129, 2007. 
[2.37] S. Sinclair, and G.G.S. Pegram," Empirical Mode Decomposition in 2-D Space and Time: A Tool for Space-Time Rainfall Analysis and Nowcasting," Hydrology and Earth System Sciences Discussions, vol.9, no.3, pp.127-137, 2005.

[2.38] S.R. Long, "Applications of HHT in Image Analysis," Hilbert-Huang Transform and Its Applications, pp.289-305, 2005.

[2.39] M.J. Mercier, N.B. Garnier, and T. Dauxois, "Reflection and Diffraction of Internal Waves Analyzed with The Hilbert Transform," Physics of Fluids, vol.20, no.8, 2008.

[2.40] M. Feldman, "Experience in The Practice of System Analysis and Industrial Application," Hilbert Transform Applications in Mechanical Vibration, John Wiley \& Sons, Ltd, Chichester, 2011.

[2.41] B. Yang, and C.S. Suh, "Interpretation of Crack-induced Rotor Non-linear Response Using Instantaneous Frequency,” Mechanical Systems and Signal Processing, vol.18, no.3, pp.491-513, 2004.

[2.42] Z. Hou, and M. Noori, “Application of Wavelet Analysis for Structural Health Monitoring," pp.946-955, 1999.

[2.43] Z. Hou, M. Noori, and R.St. Amand, "Wavelet-based Approach for Structural Damage Detection," Journal of Engineering Mechanics, vol.126, no.7, pp.677-683, 2000.

[2.44] J.N. Yang, Y. Lei, and N.E. Huang, "Hilbert-Huang Based Approach for Structural Damage Detection," Journal of Engineering Mechanics, vol.130, no.1, pp.85-95, 2004.

[2.45] J.N. Yang, S. Lin, and S. Pan, "Damage Identification of Structures Using Hilbert-Huang Spectral Analysis," Proceedings of the US-Europe Workshop on Sensors and Smart Structures Technology, 2002. 
[2.46] H. Y. Wu, et al., "Eulerian Video Magnification for Revealing Subtle Changes in the World," ACM Transactions on Graph, 2012.

[2.47] N. Wadhwa, M. Rubinstein, F. Durand, and W. T. Freeman, "Phase-based Video Motion Processing," ACM Transactions on Graph, vol.32, no.4, pp.80, 2013.

[2.48] J.G. Chen, et al., "Structural Modal Identification through High Speed Camera Video: Motion Magnification," Journal of Sound and Vibration, vol.345, pp.58-71, 2015.

[2.49] J.G. Chen, N. Wadhwa, F. Durand, W.T. Freeman, and O. Buyukozturk, ’Developments with Motion Magnification for Structural Modal Identification Through Camera Video," Dynamics of Civil Structures, vol.2, pp.49-57, 2015.

[2.50] A. Davis, et al., "Visual vibrometry: Estimating material properties from small motions in video," 2015 IEEE Conference on Computer Vision and Pattern Recognition (CVPR), pp.5335-5343, 2015. 


\section{CHAPTER 3 Impact Damage Visualization in a Honeycomb Composite Panel through Laser Inspection using Zero-lag Cross-correlation Imaging Condition}

\subsection{Introduction}

One of the distinct advantages for the widespread use of fiber reinforced composite materials in high-performance structural applications, apart from their high specific stiffness and strength, excellent fatigue and corrosion resistance, is their "tailorability": not only the shape but also the layup and material itself can be designed and optimized to meet the load requirements with lighter structural weight. Composite sandwich panels have been used extensively in stiffness-critical aircraft secondary components for improved structural efficiency and reduced maintenance cost. They consist of continuous fiber reinforced plastic face-sheets separated by low-density cores as shown in Figure 2.1. The lightweight core, usually honeycomb or foam, is typically much thicker and much lighter than the face-sheets, with the aim of increasing the bending stiffness, the damage tolerance, the thermal resistance, the resistance to vibrations and noise reduction. The stiffness and strength to weight ratios and damage tolerance of sandwich constructions are especially attractive for aerospace applications.

A critical safety issue for the design of primary composite structures is vulnerability and damage tolerance due to sudden and unpredictable loading such as foreign object impacts from bird strikes, hail, metal fragments, dropped tools, and small runway debris (e.g., Giurgiutiu and Soutis [3.1]). 


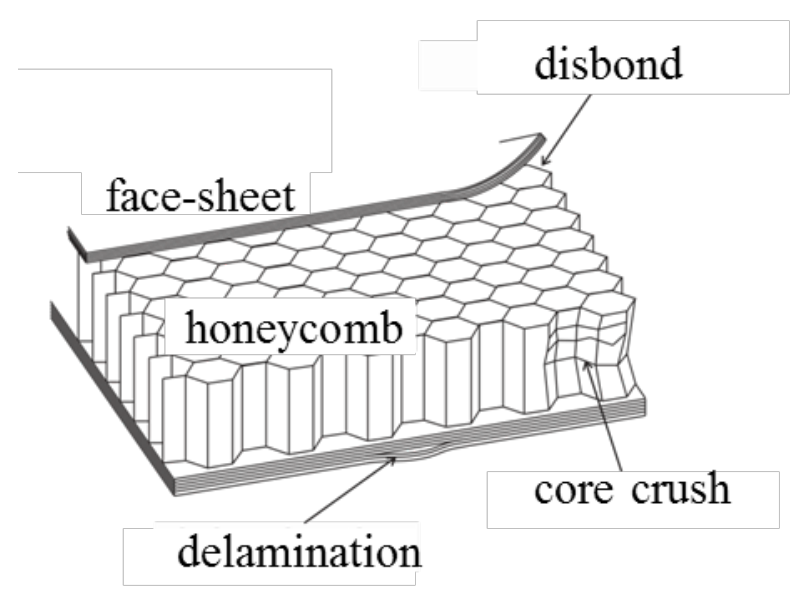

Figure 2.1. Major macro-damage failure modes of a honeycomb composite panel: core/face-sheet disbond, face-sheet delamination and core crush.

Composite structures are vulnerable to impact damage due to the brittle behavior of the matrix and to the low through-the-thickness strength. Specifically the honeycomb composite panels which this paper will focus on are also susceptible to low-velocity impact damage in which surface indentations are too small to be observed in routine inspection, yet can have significant internal macro-damage such as disbond, delamination, and core crush as illustrated in Figure 1. These panels must be designed to sustain ultimate load with barely visible impact damage (BVID). While BVID is subjective by nature, it is defined [3.2] as small damages which may not be visible during heavy-maintenance, general visual surface inspections under typical lighting conditions from a distance of $1.5 \mathrm{~m}$ or the damage causing a permanent indentation depth of $0.25 \mathrm{~mm}$ to 0.51 $\mathrm{mm}$ at the outer skin surface. The damage state establishes the design strength values to be used in analyses. These values are in compliance with the regulatory ultimate load strength requirements of FAR 25.305 [3.3]. The BVID which can be considered an evaluation of damage tolerance of the structures degrades the aspect of structural integrity which should be addressed during the design and certification phases. Structural health monitoring (SHM) and 
nondestructive inspection (NDI) can be a viable solution to this problem.

Numerous NDI methods have been utilized to detect defects or damages in composite structures. An overview of current NDI techniques for composite materials can be found in the literature (e.g., Soutis and Diamanti [3.4]). Among the NDI techniques used to evaluate composite structures, the guided wave techniques have shown good potential enabling an effective estimation of the location, size and quantification of the damage for long range inspection. Guided waves are mainly used in NDI applications in two working modes: (1) pitch-catch mode in which excitation and sensing piezoelectric elements are distant away (sparse array), spatially distributed by the structural area under evaluation, (2) pulse-echo mode in which the piezoelectric element exciting the ultrasonic signal is also used to measure any echo reflected from defect or damaged areas. A number of piezoelectric elements can also be configured as a compact array (dense phased array) to perform near pulse-echo mode. These techniques are widely used in SHM, where piezoelectric elements excite and measure guided waves while algorithms based on time-ofarrival (such as Diamanti et al. [3.5]), signal amplitude (such as Giurgiutiu, [3.6]), mode conversion (such as Okabe et al., [3.7]) enables locating structural anomalies.

In order to gather rich information about the damage area and enhance its resolution, the use of non-contact means (such as laser Doppler vibrometer (LDV), air-coupled transducers (ACTs), or electromagnetic acoustic transducers (EMATs), etc.) to scan the possible damage area of interest has been becoming a viable approach (e.g., [3.8]). Non-contact NDI can provide more detailed diagnostics once the rapid, in-service structural health monitoring (SHM) system has identified potential damage. A research group led by Prof. Staszewski was perhaps one of the very first to use the LDV for fatigue crack detection in metallic structures, conducted by Leong et al. [3.9]. A three-dimensional (3-D) scanning vibrometry (PSV-400-3D) which can capture transverse and 
in-plane measures has been recently employed by Barnoncel et al. [3.10] to detect small defects such as porosity. A review of using LDV for structural health monitoring can be found by Staszewski et al. [3.11].

Since the entire wavefield can be attained from the use of point-by-point scanning, for example using LDV, Ruzzene [3.12] employed a signal filtering technique in the wavenumber-frequency domain to separate the reflected wavefield from the incident wavefield in an aluminum plate, each of them propagating in different directions which can be readily dictated by their corresponding wave vectors. This approach clears a way to attain the reflected waves whose magnitudes are often an order of magnitude smaller than the incident wavefield, thus they are largely masked in the entire wavefield. As a result most of the damage detection methods relying on baseline subtraction; that is, subtracting the wavefield from pristine structures, can be directly applied to the acquired reflected wavefields for locating the damages. Different damage imaging techniques have been used to isolate and quantify the damage area within the guided wavefield. Using the RMS which is the square root of the averaged sum of the sensing signals squared for damage imaging after wavefield filtering was suggested by Ruzzene et al. [3.13], Zak et al. [3.14] and Kudela et al. [3.15]. An et al. [3.8] and Park et al. [3.16] developed an algorithm to isolate standing waves trapped within delaminations and disbonds from the propagating waves in the wavenumber-frequency domain. After inverting the standing wave signal from the wavenumberfrequency back into the space-time domain, a cumulative standing wave energy (CSWE) is calculated in the entire scanned domain to image the damage. Chia et al. [3.17] developed an anomalous wave propagation imaging (AWPI) method with adjacent wave subtraction to locate artificial defects introduced in a composite wing. Rogge and Leckey [3.17] developed a local wavenumber domain analysis to determine the depth of the delamination in composite structures, 
while Tian et al. [3.18] employed numerical and experimental Lamb wavefields for delamination detection in composite structures. They used wavenumber-frequency analysis and spacewavenumber analysis to determine both the dimension of the delaminated region and the plies between which the delamination occurred.

Although rapid and accurate in locating the composite defects, most of guided wave-based NDI methods do not fully visualize structural damage in complex composite structures, limited by low resolution especially when dealing with real impact damage. The methods often employ bulky contact transducers, resulting in similar limitations as the classic ultrasonic inspection techniques. In order to overcome these limitations, a laser ultrasonic NDI system is employed to acquire the full guided wavefield in plate-like structures with impact damage. The technology combines the advantages of non-contact optical methods for ultrasonic actuation and sensing and the advantages of guided wave-based inspection techniques.

The paper is organized as follows: In Section 2, a proposed fully non-contact NDI system is described in detail for capturing the entire ultrasonic guided wavefield. An experimental study in a low-velocity impacted honeycomb composite panel is conducted and delaminations and crushed core was first observed in a C-scan and followed by a more detailed X-ray CT scan of the BVID on a top view and three side views on three cross sections respectively. In Section 3, after signal filtering in the wavenumber-frequency domain, a zero-lag cross correlation (ZLCC) imaging condition in space-frequency domain originally proposed by Zhu et al. [3.20] for a permanently mounted linear array of piezoelectric wafers, are extended to the non-contact system to visualize the BVID in the honeycomb composite panel. Recently Harb and Yuan [3.21][3.22] applied the ZLCC imaging condition for imaging the damage in metals and composites using a non-contact air-coupled transducer/LDV system. He and Yuan [3.23] employed the same 
imaging condition for damage imaging in a composite plate using a single piezoelectric wafer for excitation and LDV to scan a region covering the damage. Section 4 concludes the paper with a summary of the work and their major results.

\subsection{Experimental Study}

A schematic of the NDI system similar to those in [3.14][3.15] is shown in Figure 2.2. The system combines the advantages of guided waves and optical NDI techniques: (1) it does not require contact with the structure, therefore enabling NDI in harsh environments and inaccessible areas; (2) it can be fully automated and rapid; and (3) it has potential to provide quantitative assessment of damages and/or defects.

Images of propagating guided waves are examined with the goal of identifying and assessing any defects or damage that may be present. These wavefield images can be attained through a rapid automated optical scanner using a set of galvano-mirrors that raster scans the Q-switched Nd:YAG pulse laser on a virtual two-dimensional grid on the target surface covering the damaged region. The coordinates of the grid points can be assigned based on the desired spacing between two adjacent scanning points. At each point of the interrogation grid, guided waves are generated via thermo-elastic effect and sensed at a stationary location away from the damaged region by the LDV. For convenience, the LDV points to the origin of the coordinate shown in the figure. Each time-series at the point is stored in a three-dimensional (3-D) space-time data matrix in which the first two dimensions are the spatial coordinates of the excited points while the third is the time variable. 


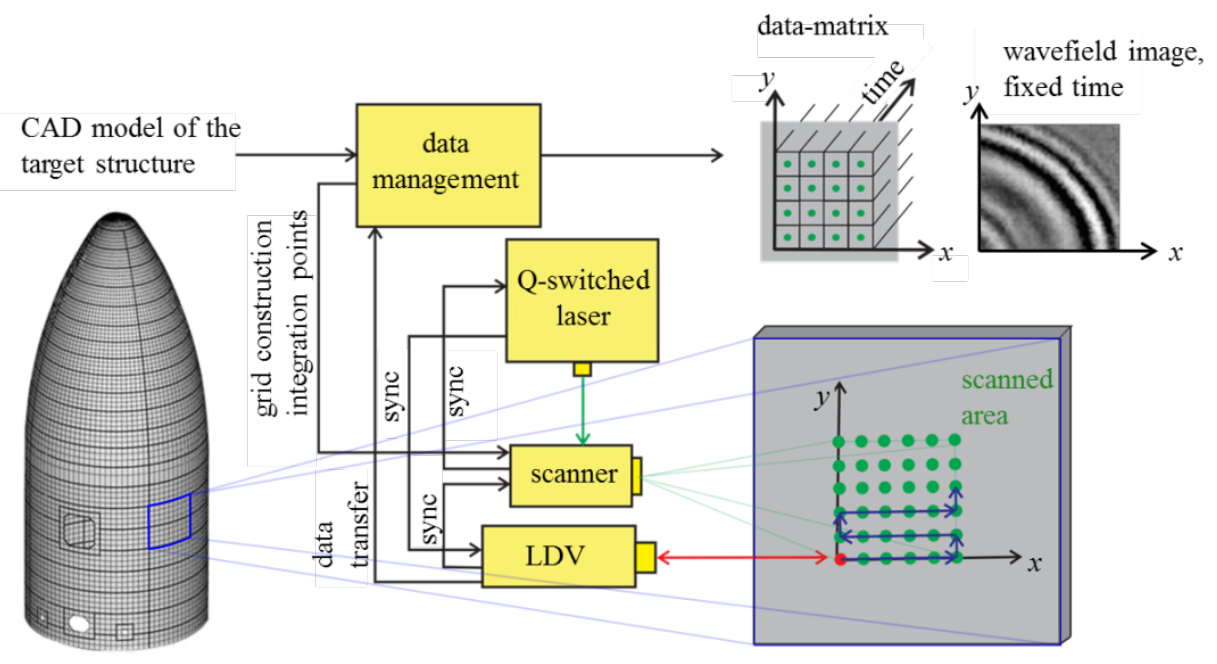

Figure 2.2. A schematic of the NDI system for imaging barely visible impact damage in honeycomb composite panel.

After assembling the 3-D data matrix, each slice of the data matrix at a fixed time corresponds to an image of the guided transient wavefield at the time instant. By the use of the Betti's reciprocity theorem extended to the linear elastodynamic case [3.24], the ultrasonic waves are equivalent to emanating at the origin and then propagate towards the damaged region where the signal is measured at every grid point within the region. The entire experimental setup is shown in the Figure 2.3. 

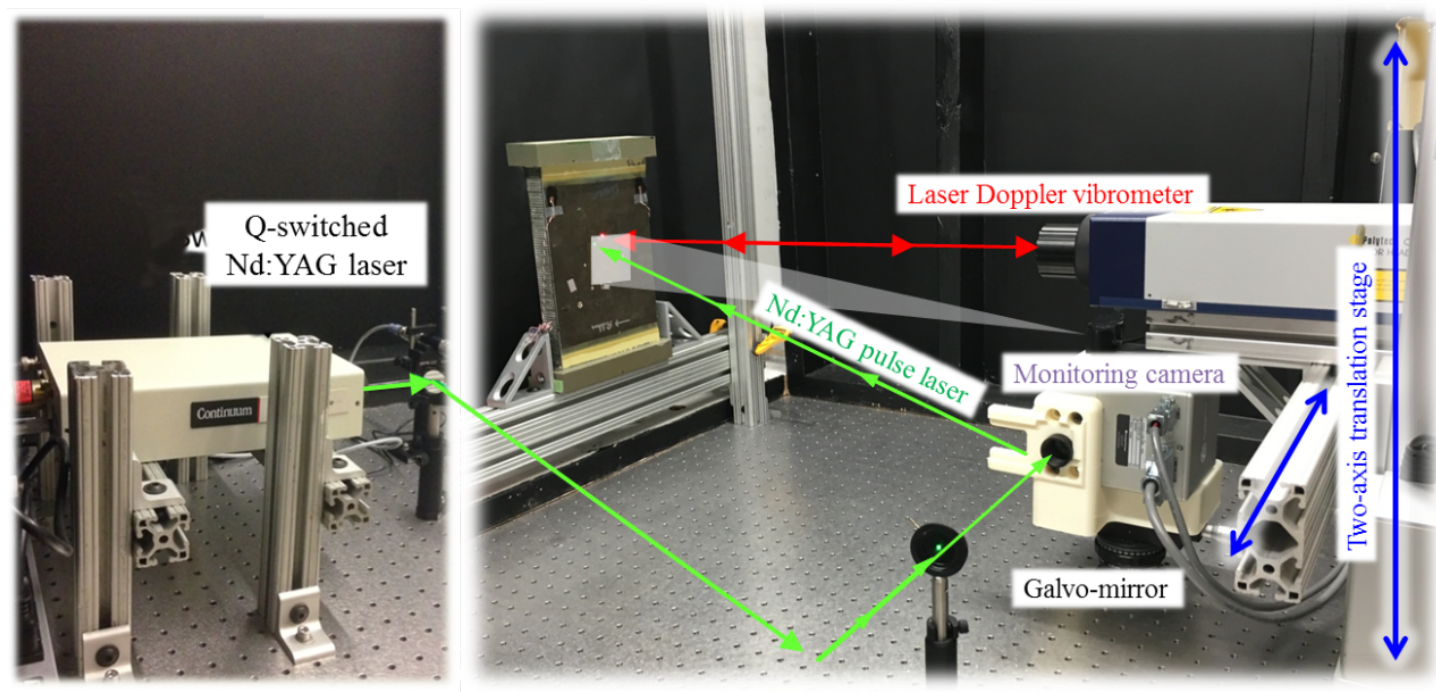

Figure 2.3. The setup of fully noncontact laser scanning system

The honeycomb composite panel as shown in Figure 2.4 has in-plane dimension: $178 \mathrm{~mm} \times 254$ $\mathrm{mm}$. The face-sheet is a 6-ply of TE-1 grade 190 type 35 carbon/epoxy with a stacking sequence of $[60 / 0 /-60]_{\mathrm{s}}$ with nominal thickness $1.14 \mathrm{~mm}$. The core is a Hexcel CR III 1/8-5052-.0007 lightweight aluminum honeycomb core with $25.4 \mathrm{~mm}$ thick. The material properties can be found in [3.25].

The panel was impacted at the center and normal to the surface with a $25.4 \mathrm{~mm}$ diameter blunt hemispherical impactor made of hardened steel and impact energy of 2 Joules. The impacted panel was bent convex slightly and the maximum dent was measured to be $0.53 \mathrm{~mm}$ deep. The resulting barely visible impact damage (BVID) may encounter delamination in the impacted facesheet, disbond between face-sheet and core, and crushed honeycomb core itself. The scan area of $50 \mathrm{~mm} \times 50 \mathrm{~mm}$ covering the impacted damage area in the panel, as shown in Figure 2.4, was first evaluated through an ultrasonic C-scan to roughly identify the dent region and show the existence of delaminations; then a detail information for the delaminated area was obtained via a $27.5 \mu m$ resolution X-ray computed tomographic (X-ray CT) scan. The X-ray CT scan will serve 
as a baseline for the comparison of the image formed by a proposed ZLCC imaging condition.

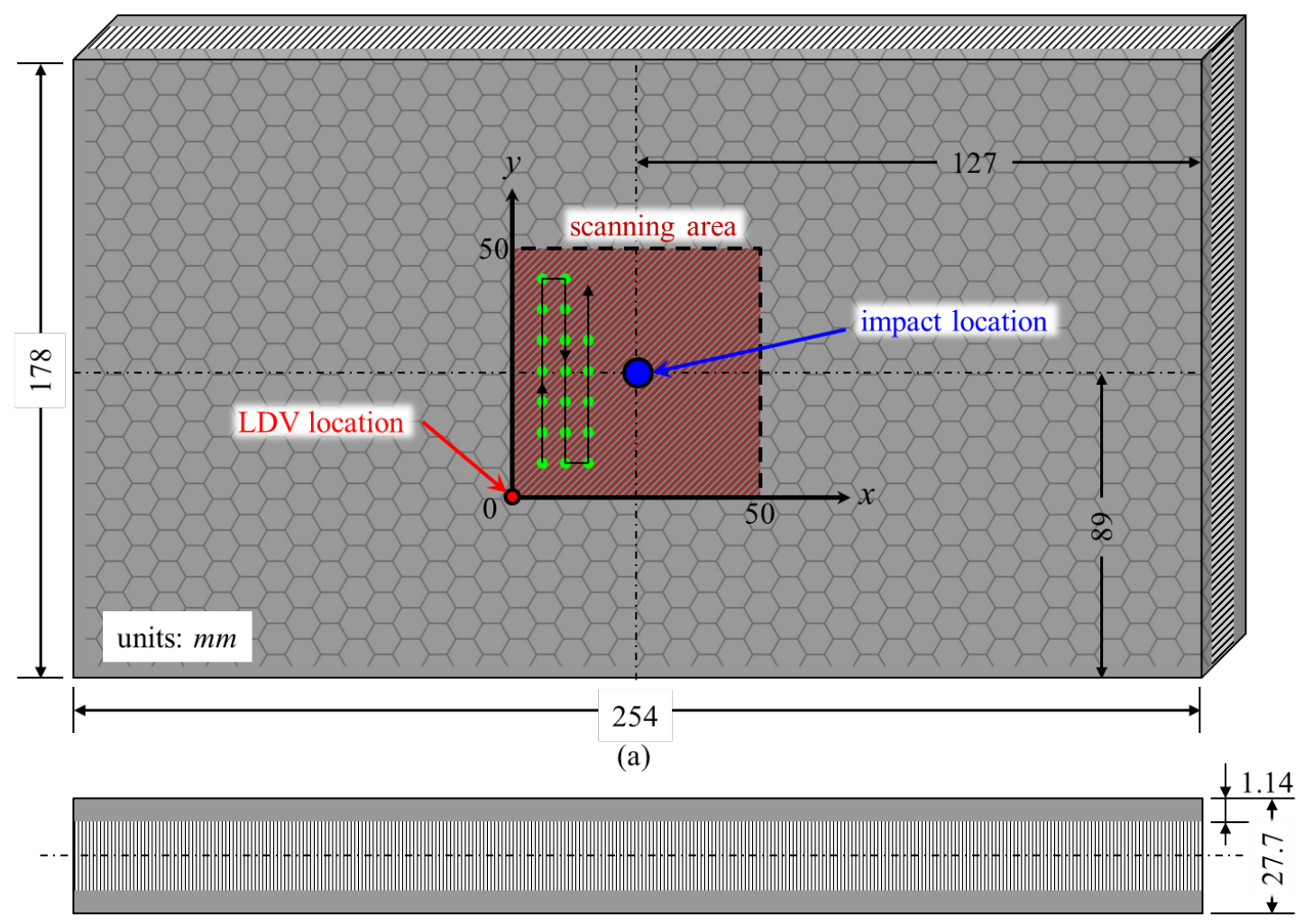

(b)

Figure 2.4. The geometry of the composite sandwich panel; (a) perspective view; (b) side view (units: $\mathrm{mm}$ )

Since the dent area was barely visible from naked eye, to precisely locate the dent and then correlate the dent with internal delaminated area, the impacted areas of the composite panels were scanned using ultrasonic C-scan mapping. An Olympus submersion transducer and a Panametrics Model 5055 PR-101 pulser receiver operating at $10 \mathrm{MHz}$ were used for the scans. Since ultrasonic signal requires liquid to travel into the targeted panel, the specimen was wetted in order to reduce impedance mismatch. Instead of submerging the entire specimen in water, a plastic column full of water between the transducer and the specimen was used as shown in Figure 2.5. The scans took data samples every $0.25 \mathrm{~mm}$ interval in a $50 \mathrm{~mm} \times 50 \mathrm{~mm}$ square region, producing images with a 
201 $\times 201$-pixel resolution. These images were interpolated in MATLAB to generate higherdefinition images. Figure 2.6(a) shows the size of the resulting dent on the surface. The images were generated by taking a slice from the C-scan in the same plane as the undented surface. The black area represents without presence of the material, thus the black ellipse that appears in the image represent where the extent of the face-sheet surface was dented toward the core from the impact. Figure 2.6(b) contains the image generated from raw scans through the thickness of the panel, and both the dent and the resulting delaminations can be observed. The cross section was taken through the deepest point of the dent; that is, the maximum damage area. Figure 2.6(c) shows in-plane cross sections through the bottom of the dent, parallel to the undented surface. Being superimposed over the image of its corresponding surface at the actual scan location, it can be helpful to show the actual impact location.

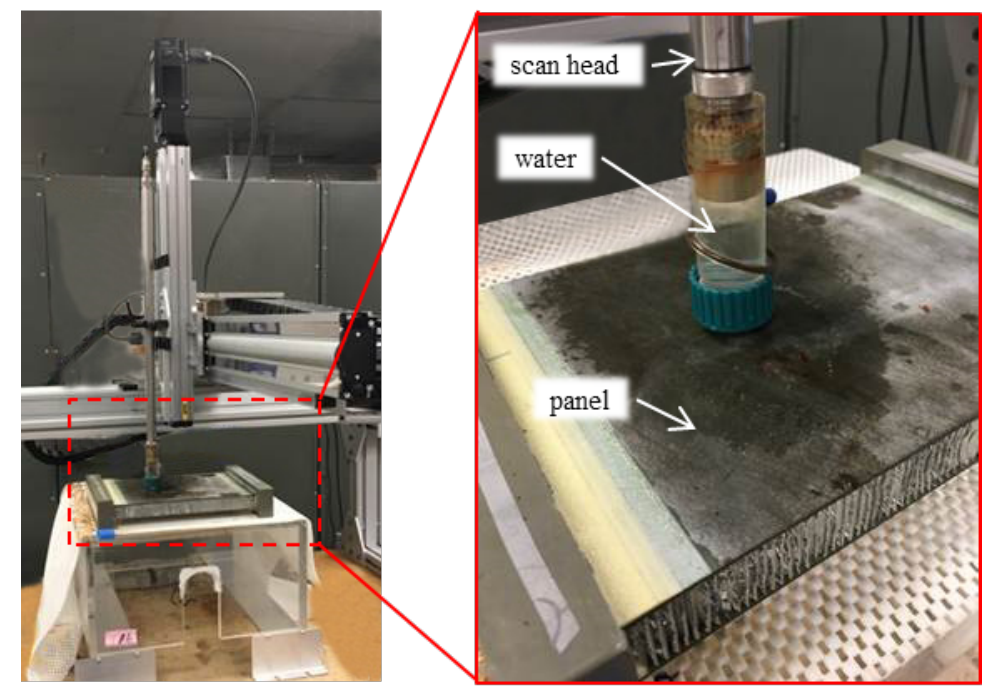

(a)

(b)

Figure 2.5. Equipment setup for ultrasonic scan of the composite panel: (a) C-scan system, (b) scanning head near the surface of the panel. 


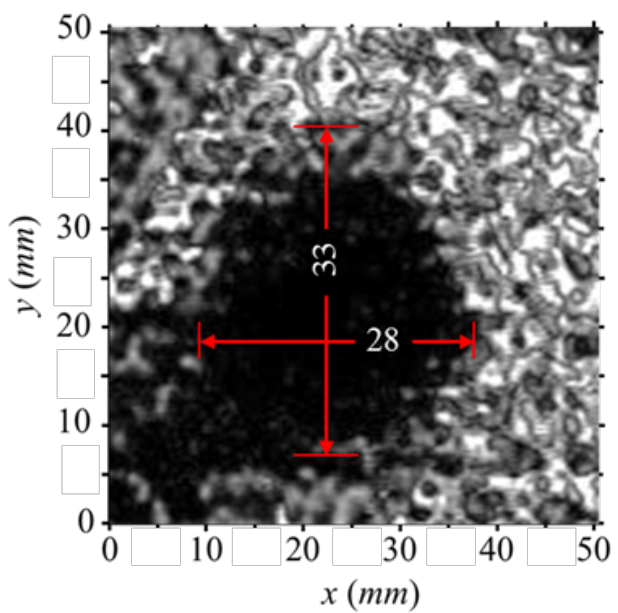

(a)

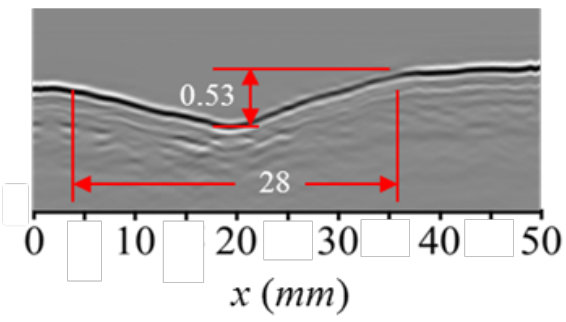

(b)

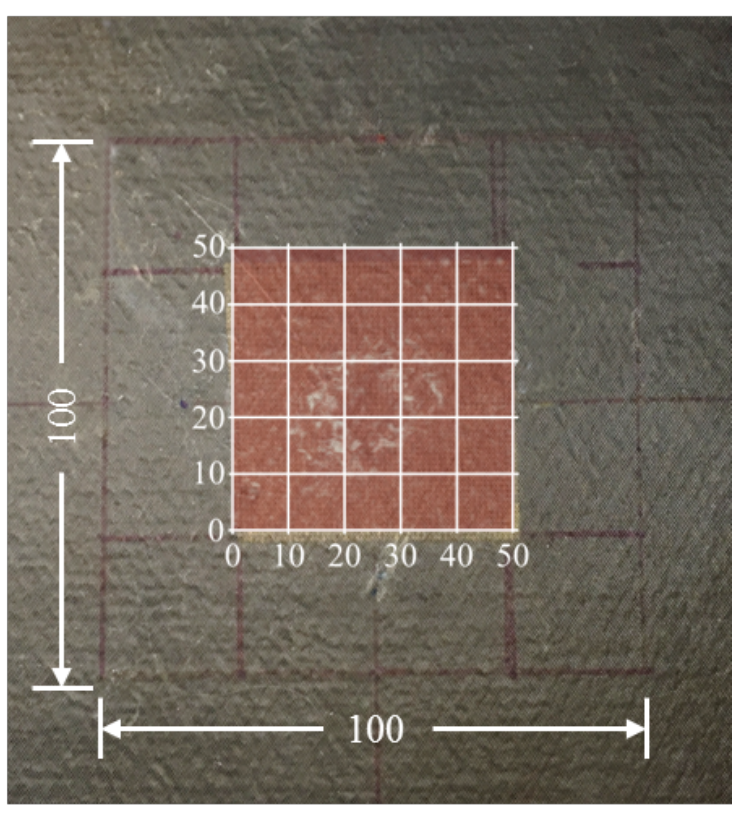

(c)

(units: $m m$ )

Figure 2.6. Ultrasonic C-scan images of (a) the dent area, (b) the cross-section view and (c) the dent location and size on the composite panel.

Figure 2.7(a) shows an image of the resulting delaminations in the face-sheet. To produce the image, the C-scan results were shown without the presence of the dented depth. A root mean square algorithm was used on the image stack of the face-sheet to highlight all of the delaminations within the face-sheet. The delamination where the layers have separated appear white due to the absence of the material. The resulting images are shown with measurements of the internal delaminations. Below the surface, a large delamination was measured to be $19 \mathrm{~mm}$ in length and a smaller delamination was measured to be around $7 \mathrm{~mm}$. Figure 2.7(b) shows the delamination image from Figure 2.7(a) superimposed onto the surface at the scan location. 


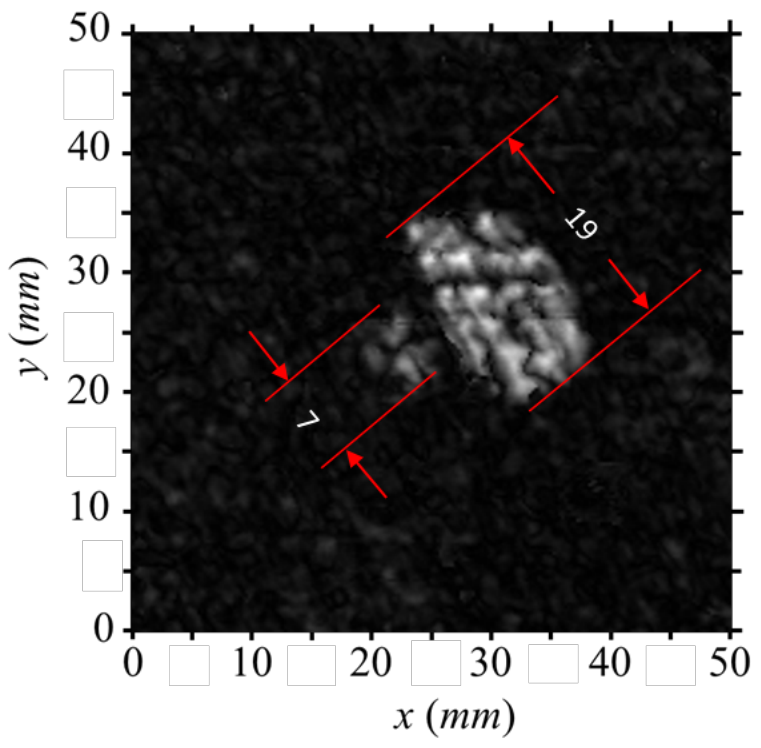

(a)

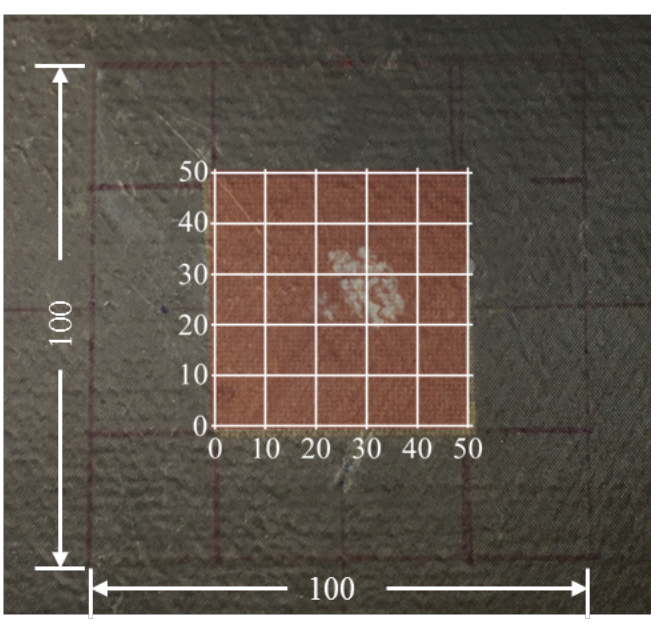

(b)

(units: $\mathrm{mm}$ )

Figure 2.7. (a) Ultrasonic C-scan images of the delamination area, and (b) the delamination location and size on the composite panel.

Ultrasonic C-scan merely showed the existence of delamination in the face-sheet, but without information of the delaminated area. The panel was then interrogated via X-ray CT scan for further evaluation. Figure 2.8 shows a set of images obtained by X-ray CT scan focusing on a plane $0.15 \mathrm{~mm}$ below from the core/face-sheet interface where the maximum projected area of delamination was reached. Due to the hardware limitation of the CT scan system, a $30 \mathrm{~mm} \times 35$ $\mathrm{mm}$ area was imaged at the impact location instead of the entire laser interrogating area $50 \mathrm{~mm} \times$ $50 \mathrm{~mm}$ using LDV. Three cross-sections side view $(A A, B B$, and $C C)$ near the delamination location were taken from the X-ray CT scan. The top view reveals two dark projected delaminated regions in the face-sheet, where the main delamination is centered around $x=23 \mathrm{~mm}, y=20 \mathrm{~mm}$ and the secondary delamination is centered around $x=17 \mathrm{~mm}, y=17 \mathrm{~mm}$. The horizontal $\mathrm{AA}$ and $B B$ sections, respectively shown at $x=23 \mathrm{~mm}$ and $x=17 \mathrm{~mm}$, display the crushed core area and show the depth of delaminations. The vertical cross-section $C C$ at $x=22 \mathrm{~mm}$ also displays a 
similar damage pattern. From the three cross-sectional images, the shape of main delamination area can be approximated as an ellipse with major and minor axis lengths, $17 \mathrm{~mm}$ and $10 \mathrm{~mm}$ respectively and oriented 105 degrees from the positive $x$-axis. Similarly, the shape of secondary delamination is approximately an ellipse with $6 \mathrm{~mm}$ and $4 \mathrm{~mm}$ in two major axes. The delamination mainly appears to be located between the fourth and fifth ply from the free top surface, although part of the delaminations migrate into the interface between the fifth and the sixth (bottom) ply. The X-ray CT scan provides more detailed information to locate the delamination area, but the size of delaminations shown in CT scan result is slightly smaller than the size shown in ultrasonic C-scan result. Because the C-scan result is the accumulated images along all the depth directions which are more comprehensive but less sensitive; however the CT scan is an image taken at one specific depth from the top surface, providing sharp and precise shape of the targeted area.

A closer examination of the X-ray CT scan also revealed (not shown here) dispersed matrix cracks in the top delaminated area near the free surface. These cracks are however of minor significance when compared to the extended delamination. No disbond was observed resulting from the impact. 


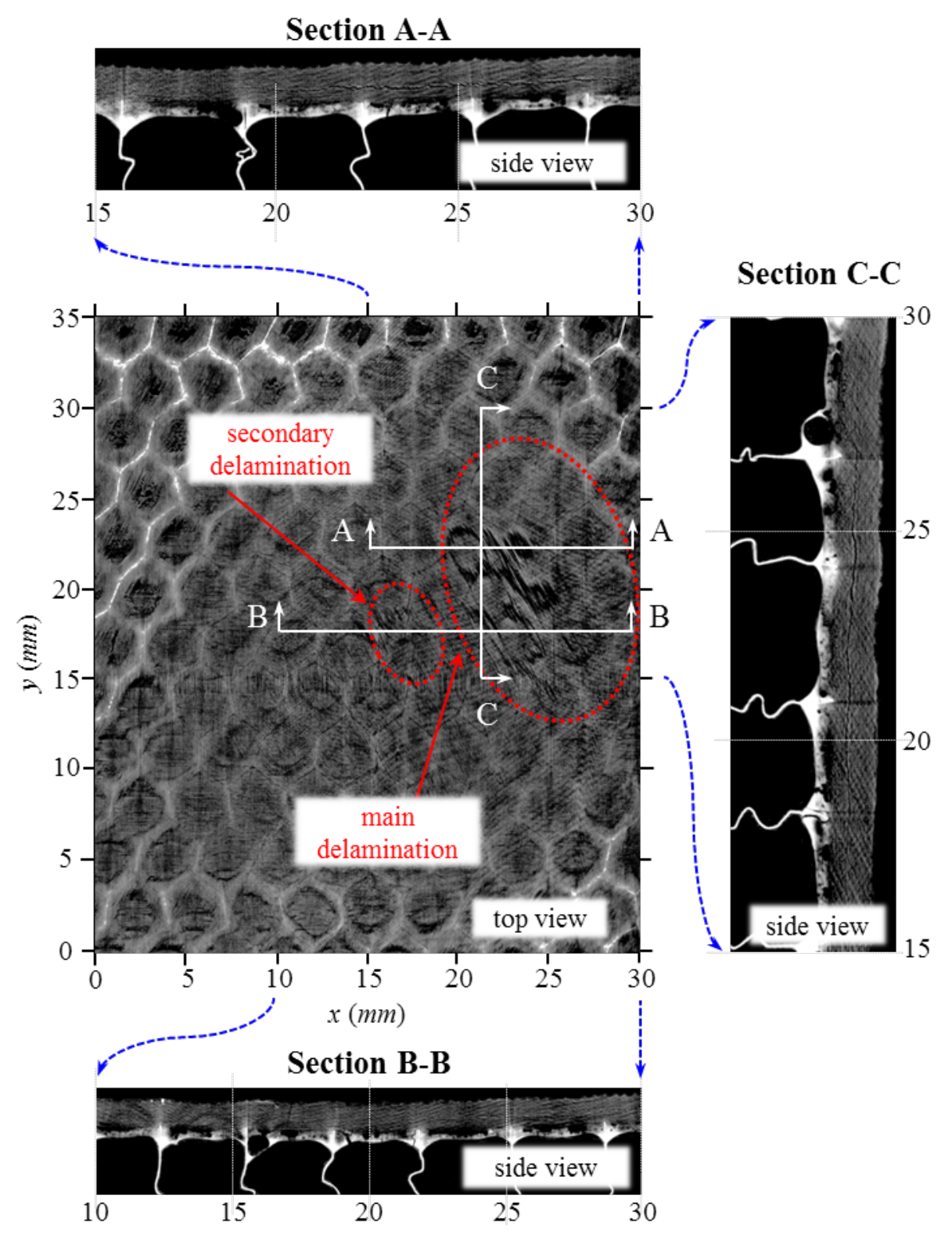

Figure 2.8. X-ray CT scan of the impacted honeycomb composite panel: (a) top view from the scanned surface, indicating two elliptical delamination regions $17 \mathrm{~mm} \times 10 \mathrm{~mm}$ and $6 \mathrm{~mm} \times 4$ $m m$, and side views from three cross-sections (b) $A A$, (c) $B B$, and (d) $C C$, revealing the depth and shape of the delaminations. 
The NDI system was then used to acquire the wavefield in the same region of the sample evaluated via X-ray CT for comparing the damage imaging. Two scans were performed: one prior to and one after the impact event. The pulse laser used to excite the ultrasonic signal was an Nd:YAG Q-switched laser producing $1064 \mathrm{~nm}$ wavelength light. The laser generates 15 pulses per second $(15 \mathrm{~Hz})$, each of which has energy of $16 \mathrm{~mJ}$ and pulse-width of 5-7 nsec. The pulse laser beam diameter was measured to be about $3.5 \mathrm{~mm}$ on the panel.

The Nd:YAG pulse laser was raster scanned on the panel surface by an optical scanner using a set of galvano-mirrors having a repeatability of $8 \mu \mathrm{rad}$ and positioned at about $500 \mathrm{~mm}$ normal from the panel. A total of $2601(51 \times 51)$ points were interrogated with spacing of $1 \mathrm{~mm}$ in both $x$ and $y$-directions. For each excited point, the out-of-plane velocity was measured though a LDV (Polytec OFV-505) stationary at the origin, as shown in the NDI schematics, Figure 2.2. The sensing laser beam on the surface is roughly around $2 \mathrm{~mm}$ diameter. In order to improve the captured signal quality and thus increase the signal to noise ratio (SNR), a retroreflective tape is applied to the interrogated area which enhances the surface reflection; in addition, for enhancing SNR, the measurement at each scanned location was averaged 25 times. The interrogating area $50 \mathrm{~mm} \times 50 \mathrm{~mm}$ can be scanned in less than 3 minutes without averaging; ideally, the scanning duration for an entire A350XWB wing, where the area is approximately $443 \mathrm{~m}^{2}$, with a spacing of $1 \mathrm{~mm}$ and a $100 \mathrm{kHz}$ pulse repetition rate from a pulsed laser can be approximately calculated as $\frac{443 \times 10^{6} \text { samples }}{100 \mathrm{kHz} \times 60 \mathrm{sec}}$, which is less than 75 minutes.

To examine the characteristics of the excitation signal generated by the pulse laser, it is convenient to measure the signal using LDV right next to the location, roughly $1-2 \mathrm{~mm}$ as shown in Figure 2.9 since the wavefield is captured from the LDV. Figure 2.9(a) shows the signal in 
time domain; the Figure 2.9(b) shows its magnitude in frequency domain. For better quality of the signal, the LDV decoder was set to VD-09 $10 \mathrm{~mm} / \mathrm{s} / V$ with maximum frequency at $250 \mathrm{kHz}$. It yielded the maximum sensitivity at frequencies around $100 \mathrm{kHz}$ shown in Figure 5(b).

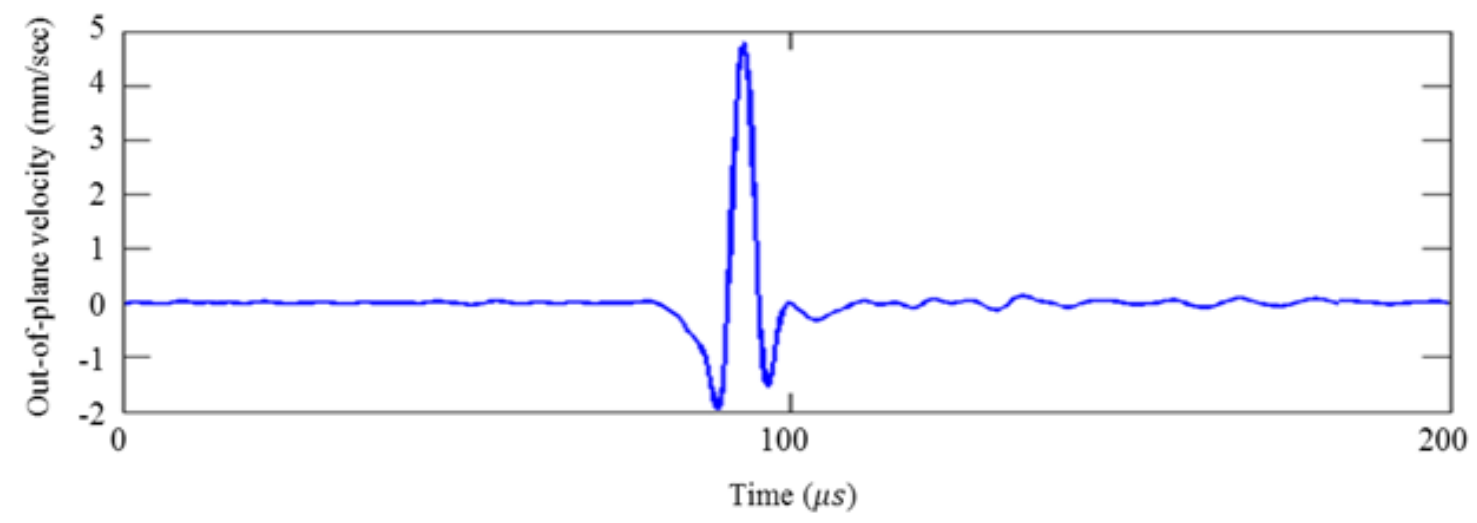

(a) Time domain

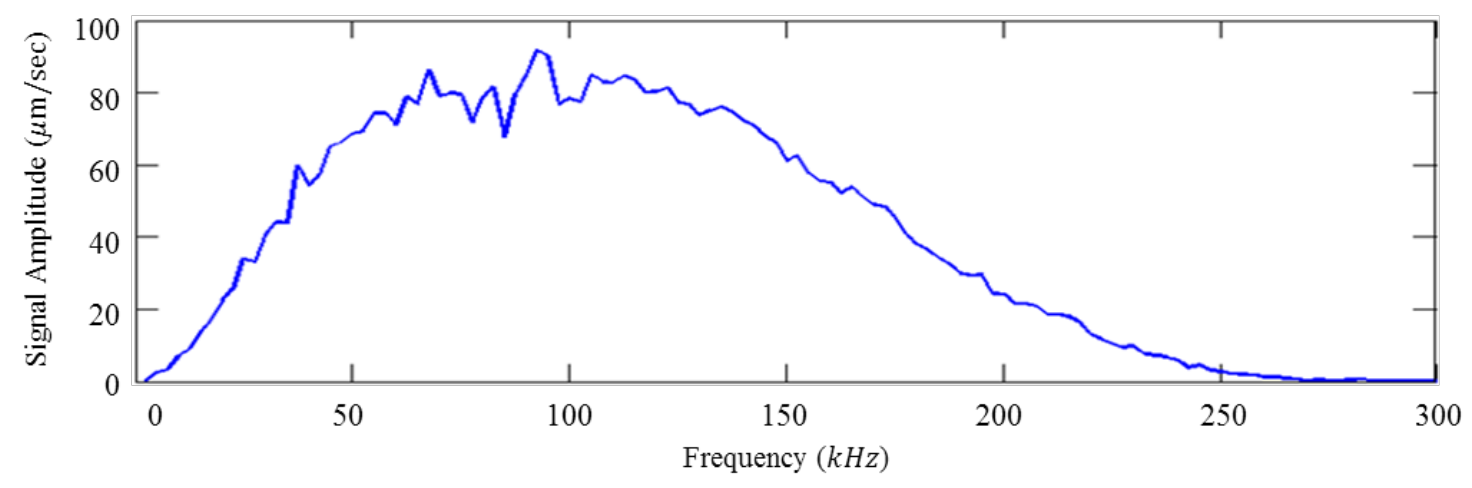

(b) Frequency domain

Figure 2.9. Excitation signal in the honeycomb composite panel generated by the pulse laser was measured by a nearby LDV.

Figure 2.10 shows the guided wavefield at $t=265 \mu$ s. Figure 2.10(a) represents the wavefield in the pristine honeycomb panel; while Figure 2.10(b) displays the wavefield after the panel is impacted. It can be observed that the ultrasonic wave propagating in the panel is scattered by the 
BVID. The effect of the damaged area on the wavefield can be initially noticed as a change of the direction of the ray-paths, as defined by normal to the wavefronts.

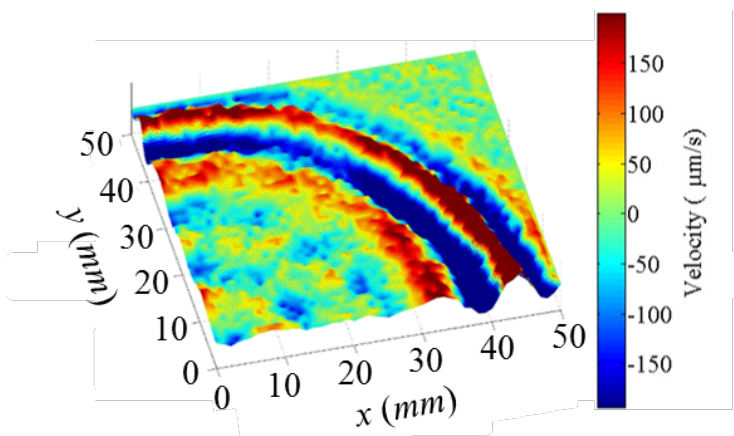

(a) prior to impact

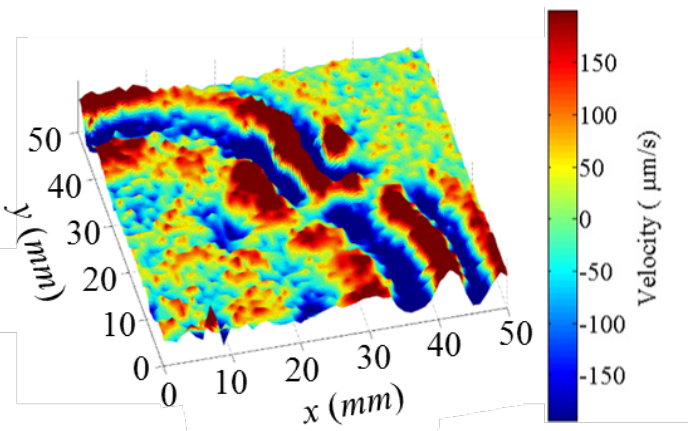

(b) after impact.

Figure 2.10. Wavefield in the honeycomb composite panel at $t=265 \mu$ s: (a) prior to impact; (b) after impact.

\subsection{BVID Visualization using Zero-Lag Cross Correlation (ZLCC) Imaging Condition}

Different damage characteristics including size, depth, shape, and orientation of the defect or damage affect guided wavefields in many different ways [3.26]. Additionally, the defect or damage can introduce new local wave modes [3.17]. Guided waves damage assessment techniques can be broadly classified in methodologies based upon guided waves mode conversion at defects and methodologies based on guided waves scattering from material and geometrical discontinuities. Guided waves propagate in different modes that depend on the frequency and the panel thickness [3.27]. NDI techniques based on guided waves mode conversion have been successfully used in pitch-catch configurations for a rapid detection of defects in composite laminates [3.28]. However, the extraction of quantitative information is complicated by the 
overlap of wave modes and the dispersive characteristics of the guided waves. In addition, data gathered from pitch-catch methodologies are limited by the sparsity of the sensor array.

A finer interrogation of the wavefield enables quantitative damage assessment based on the response of the ultrasonic wave due to wave scattered from the damage or defect. Guided waves impinging on a damage or defect region are partially reflected and refracted by the geometric discontinuity. The newly introduced geometric boundaries such as delaminations may introduce momentary standing waves due to multiple reflections within the delaminated region. These phenomena will be explained and a ZLCC imaging condition in the space-frequency domain is proposed to detect, locate and visualize damage in honeycomb composite panels.

As pointed out by Zhu et al. [3.20] for a permanently mounted linear array of piezoelectric wafers, the damage can be imaged by correlating between the incident and reflected propagating waves, both modeled by the finite difference method, called ZLCC imaging condition, at each discrete location outside of the damaged region. In the current study the imaging of the damage using ZLCC is extended to the entire interrogated area since the wavefield can be readily attained using non-contact point-by-point sensing within the interrogated area in which the waves interact with geometric discontinuities (i.e., damage boundaries) generating multiple reflections depending on the damage size, depth, and location. Two different wave phenomena occurring outside and inside the delaminated region respectively are explained as below. In the area outside the delaminated region, the forward (or incident) transient wavefield which encounters the damage boundary will generate backward (or reflected) transient wavefield. At the instant moment of the wave reflection on an arbitrary location along the damage boundary, the two propagating waves in different directions are coherent or in phase. While within the small delaminated region, a superposition of waves propagating in opposite (forward and backward) directions generates 
momentary standing waves in all directions trapped within this region. The two waves propagating in opposite directions with the same amplitude are in phase resulting in the amplitude of the standing wave being maximized. As a consequence, the mode shape of the standing wave can be extracted and visualized by performing the zero-lag cross correlation of the waves propagating in opposite directions at discrete resonant frequencies.

To employ wavenumber filtering technique, the guided wavefield is first represented in the wavenumber-frequency domain via a 3-D Fourier transform:

$$
W\left(k_{x}, k_{y}, \omega\right)=\mathcal{F}_{3 D}[w(x, y, t)]=\int_{-\infty}^{\infty} \int_{-\infty}^{\infty} \int_{-\infty}^{\infty} w(x, y, t) e^{i\left(\omega t-k_{x} x-k_{y} y\right)} d x d y d t
$$

where $\mathcal{F}()$ is the Fourier transform operator. The wavenumber map of the entire wavefield within a scan region $50 \mathrm{~mm} \times 50 \mathrm{~mm}$ at each frequency can be illustrated as Figure 2.11.Due to Nyquist sampling theorem, the maximum wavenumber value detectable is $500 \mathrm{~m}^{-1}$ because sampling spacing is $1 \mathrm{~mm}$. But the upper and lower limits of the wavenumber map shown in Figure 2.11 are set at $\pm 300 \mathrm{~m}^{-1}$ respectively for better illustration. The wavenumber map of the summation along frequency domain indicates the overall wave propagating direction, and the region is relatively large due to the wide frequency bandwidth. The decomposed wavenumber maps not only show different wavenumber values and wave propagation directions at each frequency, but also reveal the standing wave phenomenon and the sensitivity of reflected wave. At certain frequencies, two wavenumbers with identical value but opposite directions are observed in the wavenumber map. It implies the existence of standing waves induced by two waves with same frequency but opposite propagating direction. 


$$
\begin{aligned}
& A \cos (\omega t-k x)+B \cos (\omega t+k x+\phi) \\
& =\underbrace{2 A \cos \left(k x+\frac{\phi}{2}\right) \cos \left(\omega t+\frac{\phi}{2}\right)}_{\text {Standing wave }}+\underbrace{(B-A) \cos (\omega t+k x+\phi)}_{\text {Transient wave }}
\end{aligned}
$$

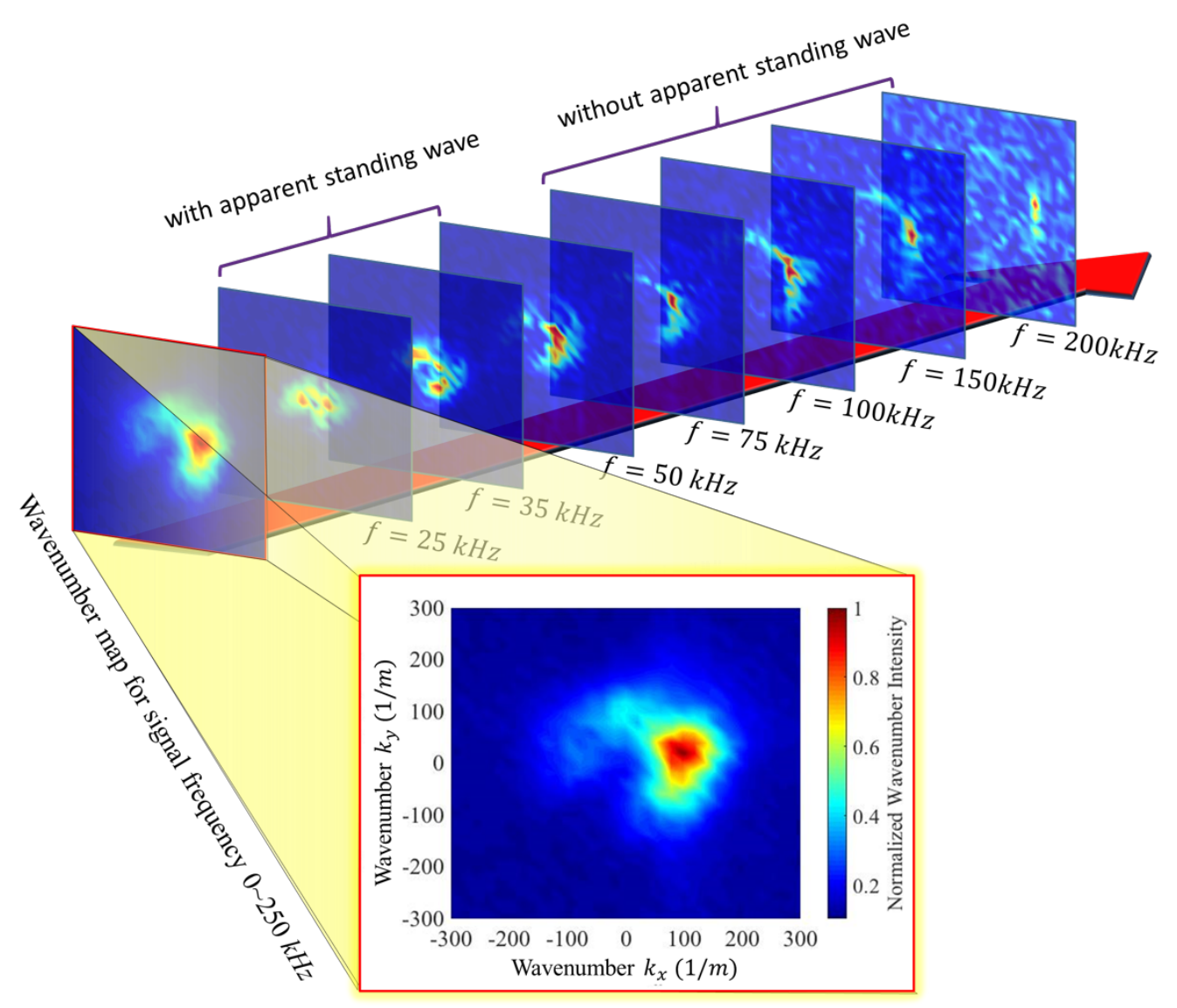

Figure 2.11. Wavenumber map at each frequency

By applying a quadrant filter to the wavenumber map at each frequency, the wavefield can be divided into four parts, which contain four separated waves propagating in each direction, as shown in Figure 2.12. The reflected wave is defined as the sum of three other quadrants than the one for the incident wave; for example, assuming the original incident wave propagates toward the direction in the first quadrant, the reflected wave may exists in either second, third or fourth 
quadrant depending on the incident angle between the incident wave and the boundary of discontinuity area.

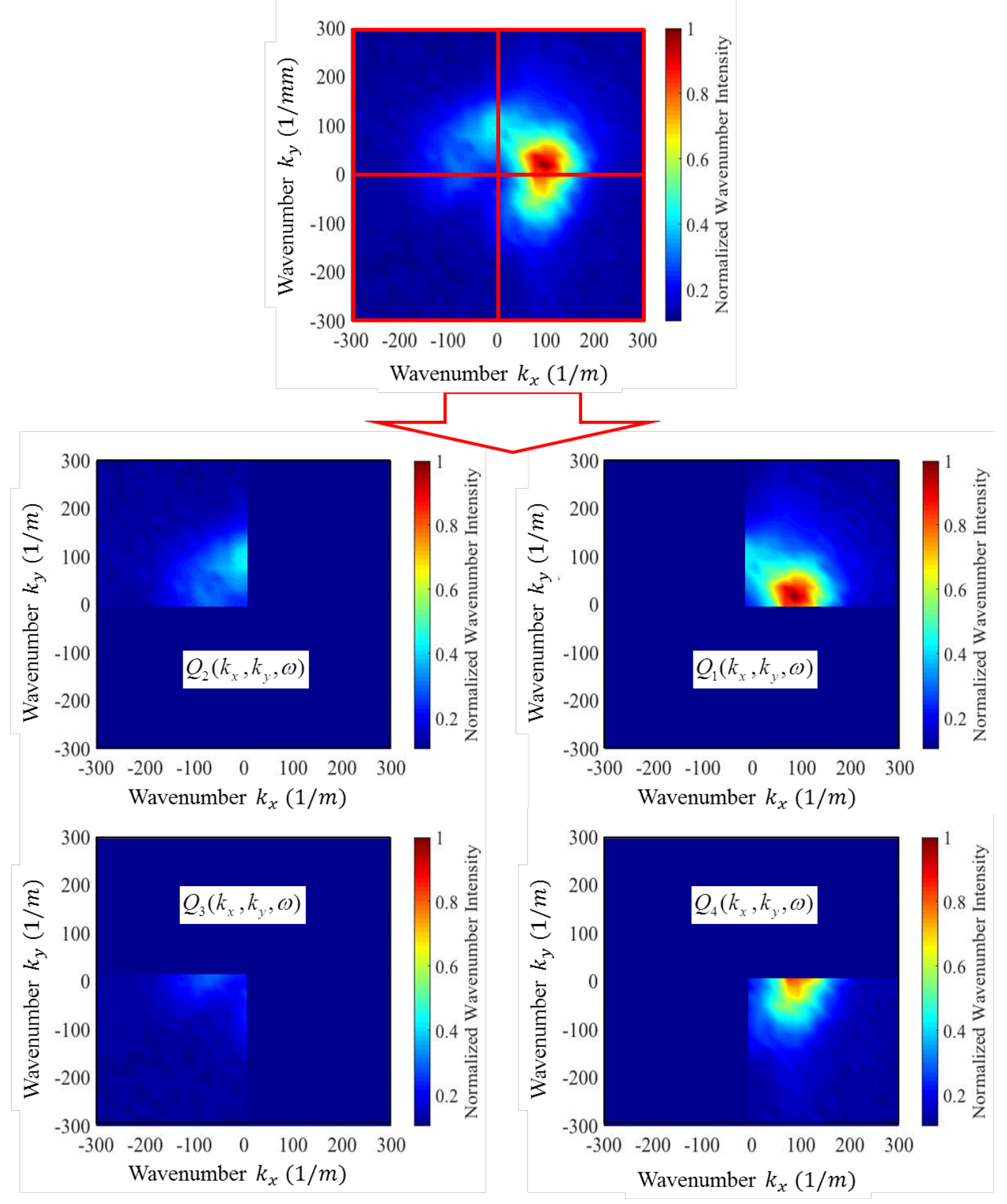

Figure 2.12. Quadrant filtered wavenumber map 
The four filtered waves can be represented as:

$$
\begin{aligned}
& Q_{1}\left(k_{x}, k_{y}, \omega\right)=\left\{\begin{array}{cl}
W\left(k_{x}, k_{y}, \omega\right), & k_{x} \geq 0, k_{y} \geq 0 \\
0, & \text { else }
\end{array} \text { for waves toward }(+x,+y)\right. \\
& Q_{2}\left(k_{x}, k_{y}, \omega\right)=\left\{\begin{array}{cl}
W\left(k_{x}, k_{y}, \omega\right), & k_{x}<0, k_{y} \geq 0 \\
0, & \text { else }
\end{array} \text { for waves toward }(-x,+y)\right. \\
& Q_{3}\left(k_{x}, k_{y}, \omega\right)=\left\{\begin{array}{cll}
W\left(k_{x}, k_{y}, \omega\right), & k_{x}<0, k_{y}<0 & \text { for waves toward }(-x,-y) \\
0, & \text { else }
\end{array}\right. \\
& Q_{4}\left(k_{x}, k_{y}, \omega\right)=\left\{\begin{array}{ccc}
W\left(k_{x}, k_{y}, \omega\right), & k_{x} \geq 0, k_{y}<0 & \text { for waves toward }(+x,-y) \\
0, & \text { else }
\end{array}\right.
\end{aligned}
$$

Therefore the total wavefield can be written as

$$
\begin{aligned}
W\left(k_{x}, k_{y}, \omega\right) & \equiv Q_{1}\left(k_{x}, k_{y}, \omega\right)+Q_{2}\left(k_{x}, k_{y}, \omega\right)+Q_{3}\left(k_{x}, k_{y}, \omega\right)+Q_{4}\left(k_{x}, k_{y}, \omega\right) \\
& \equiv W_{\text {inc }}\left(k_{x}, k_{y}, \omega\right)+W_{r e f}\left(k_{x}, k_{y}, \omega\right)
\end{aligned}
$$

where $W_{\text {inc }}\left(k_{x}, k_{y}, \omega\right)$ is characterized by an incident wave; while the remaining part of the spectrum is represented by a reflected wave $W_{r e f}\left(k_{x}, k_{y}, \omega\right)$. In this paper, the incident wave is at the first quadrant, which is clearly shown in Figure 2.12. The reflected waves can be visualized in the space-time domain by applying a 3-D inverse Fourier transform:

$$
\begin{aligned}
w_{r e f}(x, y, t) & =\mathcal{F}_{3 D}^{-1}\left[W_{r e f}\left(k_{x}, k_{y}, \omega\right)\right] \\
& =\frac{1}{(2 \pi)^{3}} \int_{-\infty}^{\infty} \int_{-\infty}^{\infty} \int_{-\infty}^{\infty} W_{r e f}\left(k_{x}, k_{y}, \omega\right) e^{-i\left(\omega t-k_{x} x-k_{y} y\right)} d k_{x} d k_{y} d \omega
\end{aligned}
$$

Figure 2.13 shows the reflected wavefield at $t=265 \mu$ s after employing the wavenumber filtering technique filtered from the total wavefield shown in Figure 6, without the use of subtraction of 
the incidence wavefield (i.e., wavefield prior to impact in Figure 6(a)) from the wavefield after impact shown in Figure 6(b). Although the result from the wavenumber filtering analysis clearly shows the presence of the damage as a result of the scattered wavefield, using the scattered wave analysis does reveal the damage location, but not quantitative information about its damage size.

The root mean square (RMS) based method is considered as the simplest damage imaging method for quantitatively showing the damage size and location from the guided wavefield. The RMS map is generated by calculating the square root of the average sum of the magnitude of the wavefield during the sampling period as shown in Eq. (3.3). Figure 2.14 shows the normalized RMS value of the out-of-plane velocity in entire time history at each grid location forming a color map. The map gives a rough estimate of the delamination region.

$$
R M S(x, y)=\sqrt{\frac{\sum_{i=1}^{N} w^{2}\left(x, y, t_{i}\right)}{N}}
$$

where $w(x, y, t)$ is the magnitude of the wavefield at location $(x, y)$ and time $t$, and $N$ is the number of time samples.

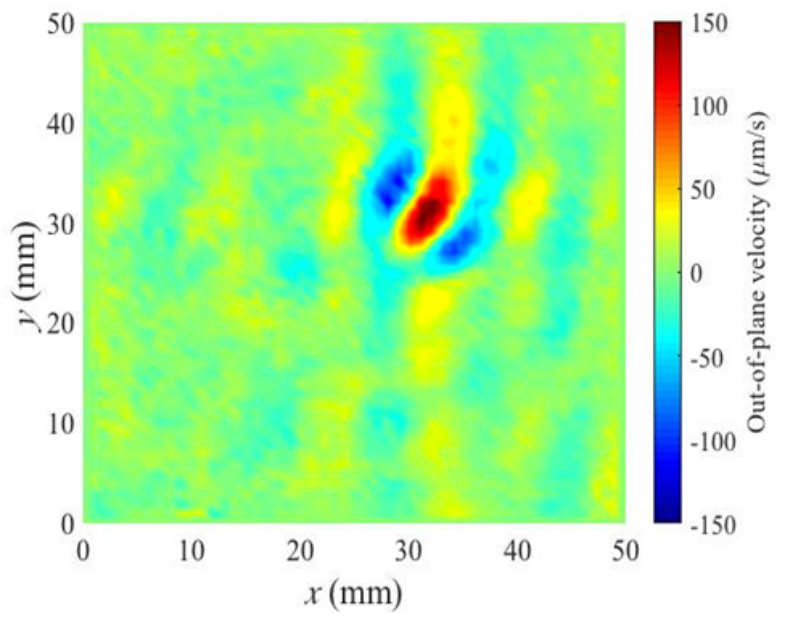

Figure 2.13. Filtered reflected wavefield at $t=265 \mu s$. 


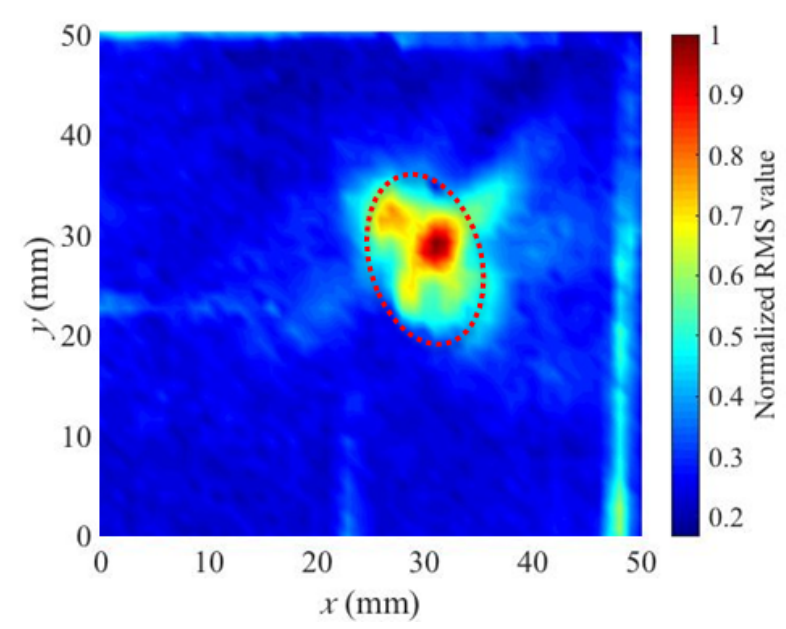

Figure 2.14. Damage image using a simple RMS of out-of-plane velocity response

After the incident wave and reflected wave have been separated by the quadrant wavenumber filter, a zero-lag cross correlation (ZLCC) imaging condition is conveniently employed to image the BVID region in the frequency domain at each point of the wavefield including the impacted region:

$$
I(x, y, \omega)=\mathcal{F}_{2 D}^{-1}\left[W_{\text {inc }}\left(k_{x}, k_{y}, \omega\right)\right] \cdot \mathcal{F}_{2 D}^{-1}\left[W_{r e f}^{*}\left(k_{x}, k_{y}, \omega\right)\right]
$$

where $\mathcal{F}_{2 D}^{-1}$ represents inverse 2-D spatial Fourier transform.

The multiple reflections of the refracted waves from the incident waves within the delamination region introduce momentary standing waves confined within the delaminated region. Distinct discrete frequencies dictate different local resonance modes of the damaged region. The decomposed ZLCC results are shown in Figure 2.15; apparently, the lower frequency bandwidth possesses better SNR due to higher sensitivity of the wave reflection, as the wavenumber maps previously shown in Figure 2.11. The ZLCC result shows the clearest damage region at $25 \mathrm{kHz}$, which is consistent with the frequency where the first standing wave (resonance frequency) 
occurs. Moreover, some other ZLCC results are clearly observed at certain frequencies, indicating that these ZLCC results reveal standing waves with higher modes at their local resonant frequencies.

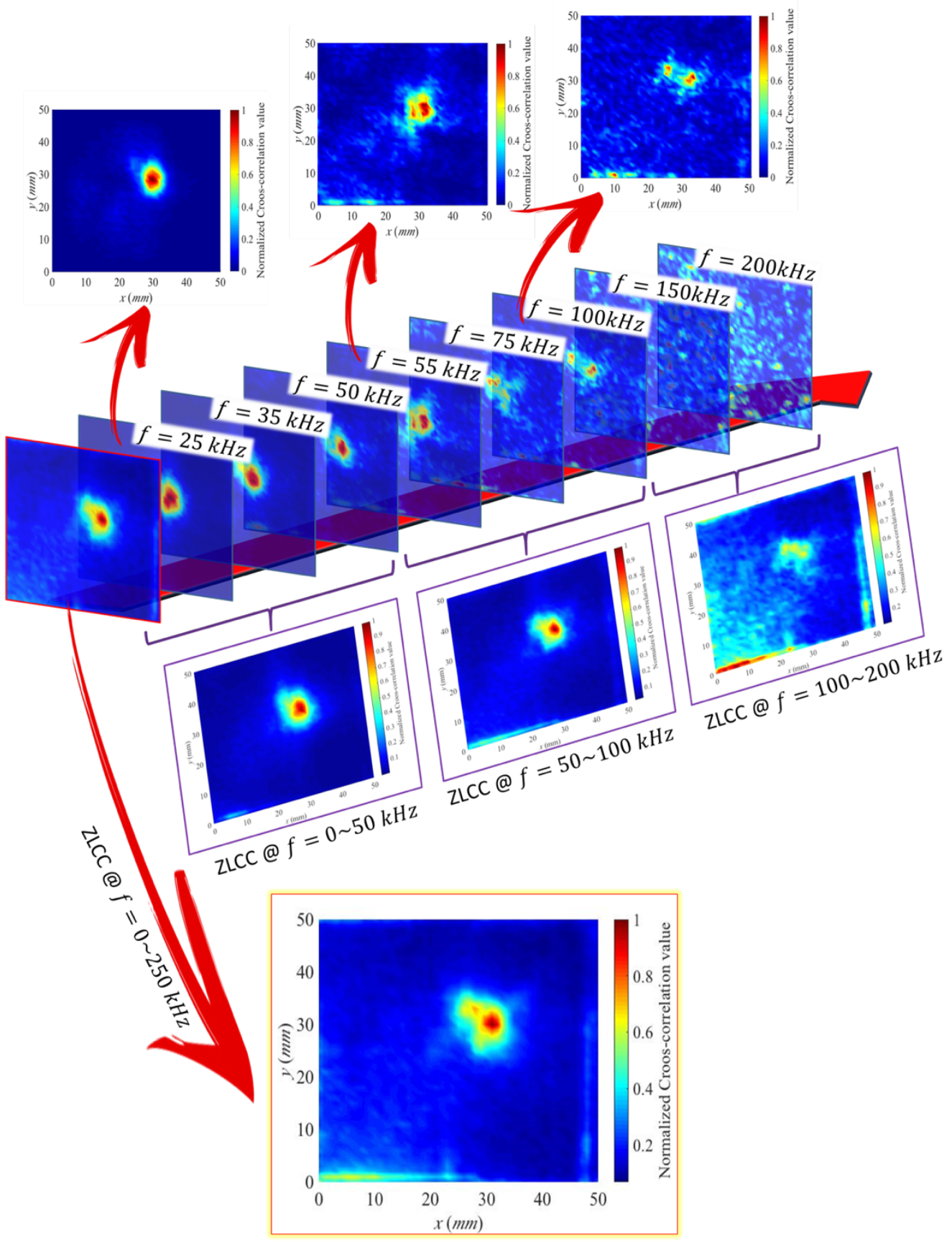

Figure 2.15. The decomposed damage imaged using ZLCC imaging condition at each frequency 
Figure 2.16 shows the damage imaged using the ZLCC imaging condition at three frequencies yielding higher image intensities and highlights the corresponding first two resonance modes of the main delaminated area. The lowest fundamental mode is shown at a frequency of about 25 $\mathrm{kHz}$, Figure 2.16(a), while the second mode is appeared at a frequency of about $55 \mathrm{kHz}$ in Figure 2.16(b). The artifacts at the boundaries of the images shown in Figure 2.16(b) may be a consequence of the edge effect of the discrete Fourier transform applied to finite domains. Figure 2.16(c) illuminates the secondary delaminated area as well as higher mode in main delaminated area.

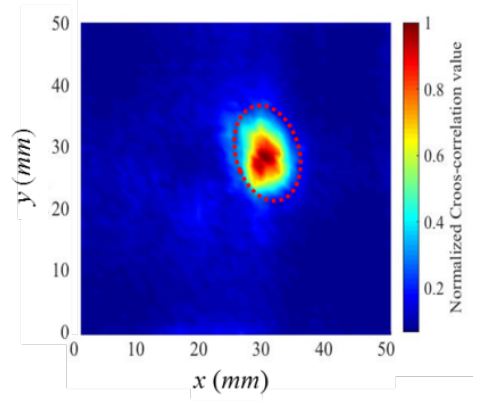

(a) $f=25 \mathrm{kHz}$

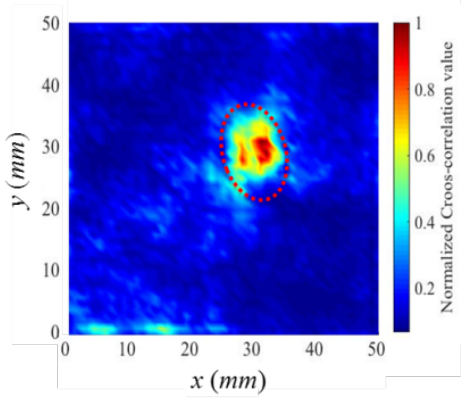

(b) $f=55 \mathrm{kHz}$

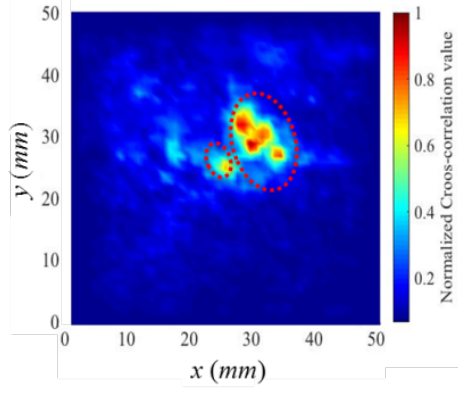

(c) $f=80 \mathrm{kHz}$

Figure 2.16. The damage imaged using ZLCC imaging condition at

$$
\text { (a) } 25 \mathrm{kHz} \text {, (b) } 55 \mathrm{kHz} \text {, (c) } 80 \mathrm{kHz} \text {. }
$$

The wavenumber maps at each frequency for the ZLCC results above are shown in Figure 2.17. Wavenumbers with the same value but located at different quadrants lead to the standing wave phenomenon occurred at the delaminated area. 


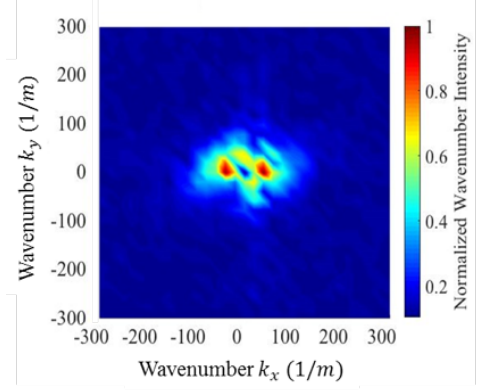

(b) $f=55 \mathrm{kHz}$

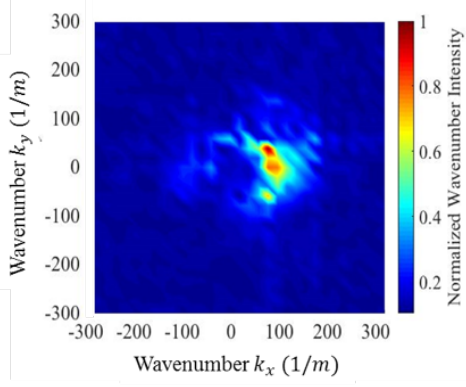

(c) $f=80 \mathrm{kHz}$

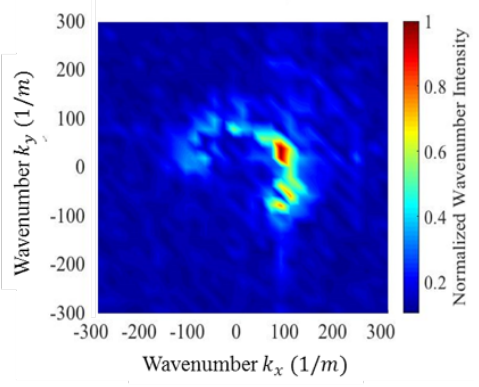

(c) $f=80 \mathrm{kHz}$

Figure 2.17. The wavenumber map showing standing wave phenomenon at

(a) $25 \mathrm{kHz}$, (b) $55 \mathrm{kHz}$, (c) $80 \mathrm{kHz}$.

A simplified finite element analysis (FEA) was conducted using ABAQUS for the verification of local resonant frequencies for verifying standing wave phenomenon. The material properties used for simulation are listed in Table 2.1 The pristine laminate, which is a six-layer laminate [60/0/$60]_{\mathrm{s}}$ was modeled within the scanned area $50 \mathrm{~mm} \times 50 \mathrm{~mm}$ and coupled with the honeycomb core to support the laminate underneath. A 105 degree off-axis skewed elliptic damaged region with major and minor axis lengths, roughly $17 \mathrm{~mm}$ and $10 \mathrm{~mm}$ respectively, was modeled with a upper (above the delamination) delaminated four-layer laminate $\left[60 / 0 /-60_{2}\right]$ and the lower (below the delamination) two-layer laminate [0/60] coupled with honeycomb, and two delaminated laminates were connected with the pristine laminate at the boundary of damage. All the laminates are meshed using an S4R shell element with $1 \mathrm{~mm}$ seeding size, leading to total 3019 elements. The honeycomb model was created as the geometry provided in the datasheet for Hexcel CR III 1/8-5052-.0007 using a 3-D element C3D10 with $2 \mathrm{~mm}$ seeding size, which yields 95829 elements in the simulation. 
Table 2.1. Material Properties of TE-1 Grade 190 Type 35 Carbon/Epoxy Tape [3.25]

\begin{tabular}{|c|c|c|c|}
\hline \multicolumn{4}{|c|}{ Elastic Properties } \\
\hline$E_{11}$ & $142.00 \mathrm{GPa}$ & $v_{12}$ & 0.34 \\
\hline$E_{22}$ & $7.8 \mathrm{GPa}$ & $v_{13}$ & 0.34 \\
\hline$E_{33}$ & $7.8 \mathrm{GPa}$ & $v_{23}$ & 0.40 \\
\hline$G_{12}$ & $4.0 \mathrm{GPa}$ & & \\
\hline$G_{13}$ & $4.0 \mathrm{GPa}$ & & \\
\hline$G_{23}$ & $2.8 \mathrm{GPa}$ & & \\
\hline
\end{tabular}

Due to the complicated fracture type and damage contour, the accuracy in simulation of higher mode requires details in damage information which is not currently available from the ultrasonic C-scan and X-ray CT scan results. From the FEA result, the fundamental mode occurs at approximately $24 \mathrm{kHz}$, which is consistent with the experimental result $f=25 \mathrm{kHz}$, and the second mode from the experiment result can also be shown from the FEA, as shown in Figure 2.18; moreover, the second local resonant frequency from FEA is around $55 \mathrm{kHz}$ which is almost the same as the experimental value. 


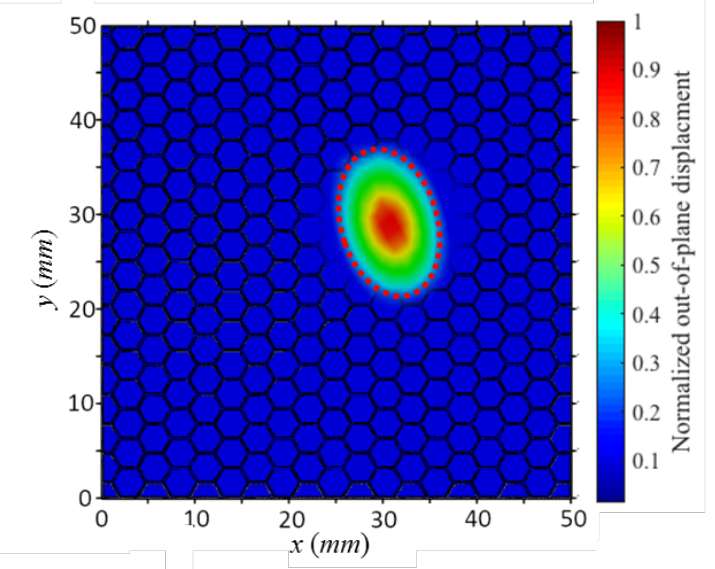

(a)

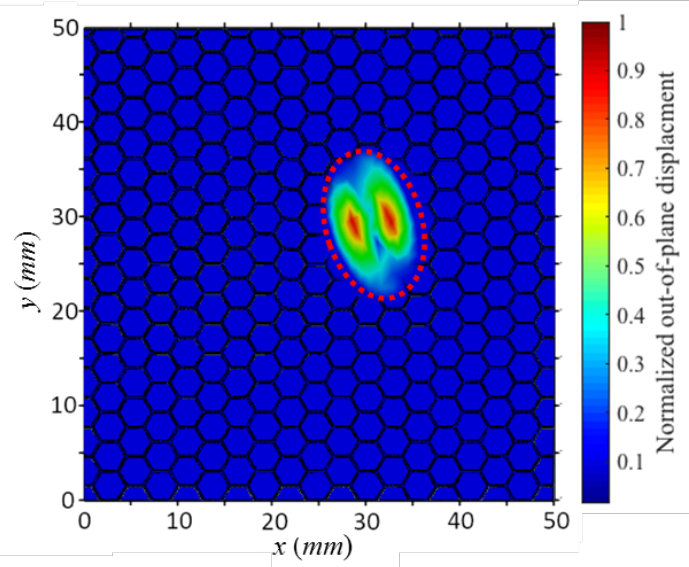

(b)

Figure 2.18. Local resonant result from FEA

(a) First mode around $24 \mathrm{kHz}$; (b) Second mode around $55 \mathrm{kHz}$.

The final image can be readily formed by superposing the image from each frequency given by

$$
I(x, y)=\sum_{\omega=\omega_{i}}^{\omega_{f}} W_{i n c}(x, y, \omega) W_{r e f}^{*}(x, y, \omega)
$$

where $\omega_{\mathrm{i}}$ and $\omega_{\mathrm{f}}$ are the lower and upper limits of the interested angular frequency spectrum of the wavefield measured. In this case, the minimum and maximum frequency limits, $f=\omega / 2 \pi, 10$ $\mathrm{kHz}$ and $250 \mathrm{kHz}$ are used respectively.

Figure 2.19 shows the damage image using the ZLCC over the frequency range $0-50 \mathrm{kHz}$. The image for the main delamination is in very good agreement with the delaminated area marked in red dotted line from the X-ray CT scan. This image is much sharper than that from RMS map shown in Figure 2.14. 


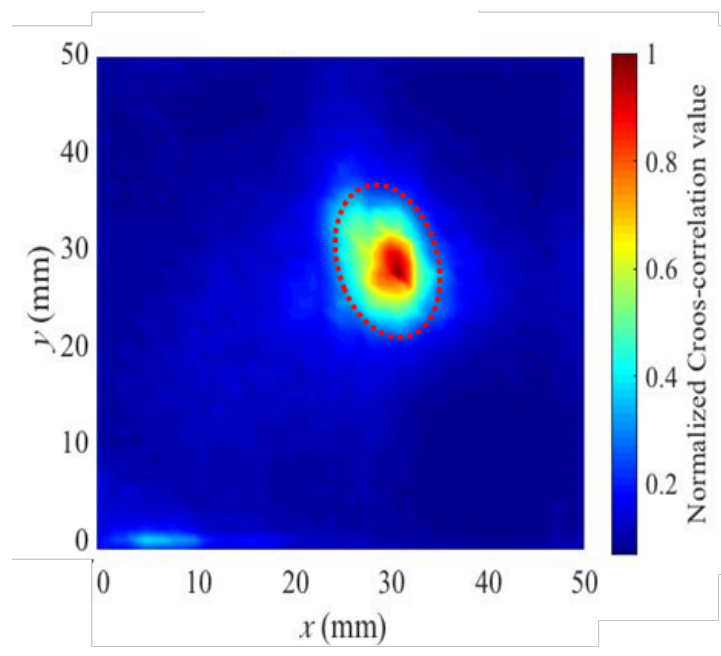

Figure 2.19. The damage image using ZLCC imaging condition summed over the frequency range, $0-50 \mathrm{kHz}$.

The ZLCC with higher frequencies yields not only the higher mode for standing wave but also better sensitivity for smaller defects. Figure 2.20 shows the damage image using the ZLCC over the frequency range 80-95 $\mathrm{kHz}$. The result reveals higher mode in the main delaminated area as well as the secondary delaminated area.

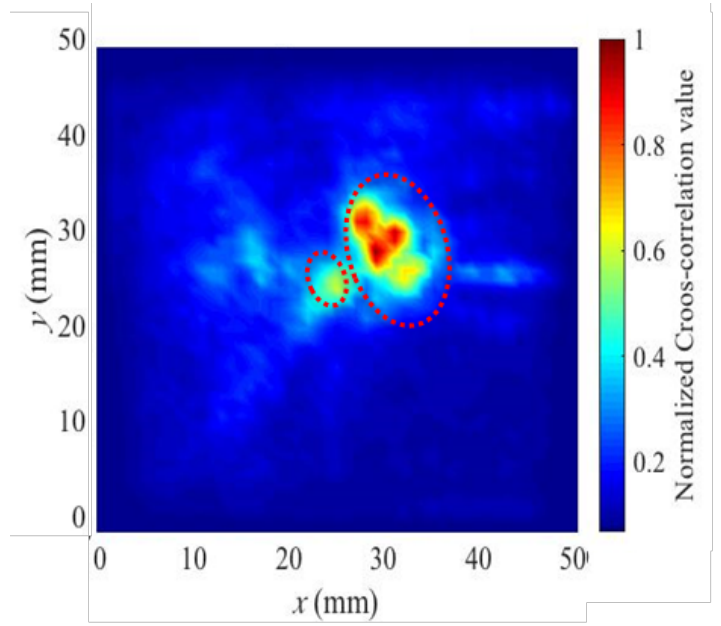

Figure 2.20. The damage image using ZLCC imaging condition summed over the frequency range, 80-95 $\mathrm{kHz}$. 


\subsection{Conclusions for ZLCC Imaging Condition}

A fully non-contact laser-based NDI system was developed with the goal of assessing hidden barely visible impact damages (BVIDs) and flaws in advanced composite structures for aerospace applications. The hardware consists of a Q-switched Nd:YAG pulse laser to generate ultrasonic signal into a quasi-isotropic [60/0/-60] shoneycomb composite panel, a set of galvano-mirrors to rapidly raster scan the Q-switched laser, and a laser Doppler vibrometer (LDV) to measure the ultrasonic wavefield guided by the structural boundaries. The ultrasonic signals acquired by the NDI system enables acquiring the wavefield guided by the structure.

A zero-lag cross correlation (ZLCC) imaging condition in space-frequency domain is proposed to identify and visualize delamination damage in the honeycomb composite panel. A signal filtering technique performed in the wavenumber-frequency domain permits to isolate the wave vectors from the delamination by filtering out the forward wavefields. The ZLCC, extended to the entire wavefield in the interrogated area, enables extraction of momentary standing waves formed and trapped within the delaminated region, in addition to cross-correlating the reflected wavefields from the geometric discontinuities (damage boundaries) and the incident guided wavefields.

The NDI system has been employed to study a BVID with a skewed $17 \mathrm{~mm} \times 10 \mathrm{~mm}$ ellipseshaped delamination in the honeycomb composite panel under impact energy 2 Joules. Deeper delaminations occurred between the fourth and fifth layers closer to the core/face-sheet interface, some of them migrating into the fifth and sixth layer interface. Matrix cracks mainly appeared in the subsurface from the top face-sheet surface. No disbond was observed. The system successfully acquired the guided wavefield in the scanned region. Although the scattered 
wavefield analysis operated in the space-time clearly reveals the presence of a discontinuity in the wavefield, it did provide rough information about the damage location, but not the damage size. The ZLCC algorithm operated in the space-frequency domain, on the other hand, provided accurate location and extent of the impact delamination damage. The image of the delaminated region was in very good agreement with that from an X-ray CT scan. The ZLCC imaging condition also enabled identification of the first two resonant modes moderately accurate accurately for the delaminated area which can be used for characterizing the damage region. The ZLCC operated at higher frequencies can effectively detect minor and smaller defects which may be ignored and hard to be identified. 


\subsection{References}

[3.1] V. Giurgiutiu and C. Soutis, "Enhanced Composites Integrity Through Structural Health Monitoring," Applied Composite Materials, vol. 19, no. 5, pp. 813-829, 2012.

[3.2] A. J. Fawcett and G. D. Oakes," Boeing Composite Airframe Damage Tolerance and Service Experience," NIAR/FAA Workshop for Composite Damage Tolerance and Maintenance, 2006.

[3.3] H. Razi and S. Ward, "Principles for Achieving Damage Tolerant Composite Primary Aircraft Structures,"11th DOD/FAA/NASA conference on Fibrous Composites in Structural Design, Fort Worth, TX, 1996.

[3.4] C. Soutis and K. Diamanti, "Structural Health Monitoring Techniques for Aircraft Composite Structures," Progress in Aerospace Sciences, vol. 46, no. 8, pp. 342-352, 2010.

[3.5] K. Diamanti, C. Soutis, and J. M. Hodgkinson, "Non-destructive Inspection of Sandwich and Repaired Composite Laminated Structures," Composites Science and Technology, vol. 65, no. 13, pp. 2059-2067, 2005.

[3.6] V. Giurgiutiu, "Tuned Lamb Wave Excitation and Detection with Piezoelectric Wafer Active Sensors for Structural Health Monitoring," Journal of Intelligent Material Systems and Structures, vol. 16, no. 4, pp. 291-305, 2005.

[3.7] Y. Okabe, K. Fujibayashi, M. Shimazaki, H. Soejima, and T. Ogisu, "Delamination Detection in Composite Laminates Using Dispersion Change Based on Mode Conversion of Lamb Waves," Smart Materials and Structures, vol. 19, p. 115013, 2010.

[3.8] Y. K. An, B. Park, and H. Sohn, "Complete Noncontact Laser Ultrasonic Imaging for Automated Crack Visualization in a Plate," Smart Materials and Strutcures, vol. 22, no.22, p. $025022,2013$. 
[3.9] W. H. Leong, W. J. Staszewski, B. C. Lee, and F. Scarpa, "Structural Health Monitoring using Scanning Laser Vibrometry: III. Lamb Waves for Fatigue Crack Detection," Smart Materials and Structures, vol. 14, no. 6, pp. 1387-1395, 2005.

[3.10] D. Barnoncel, W. J. Staszewski, J. Schell and P. Peres, "Damage Detection in Reusable Launch Vehicle Components using Guided Ultrasonic Waves and 3-D Laser Vibrometry," Proc. of SPIE, vol. 8695, pp. 86950D-1 - 86950D-8, 2013.

[3.11] W. J. Staszewski, R. B. Jenal, A. Klepka, M. Szwedo, and T. Uhl, "A Review of Laser Doppler Vibrometry for Structural Health Monitoring Applications," Key Engineering Materials, vol. 518, pp. 1-15, 2012.

[3.12] M. Ruzzene, "Frequency-wavenumber Domain Filtering for Improved Damage Visualization," Smart Materials and Structures, vol. 16, no. 6, pp. 2116-2129, 2007.

[3.13] M. Ruzzene, S. M. Jeong, T. E. Michaels, J. E. Michaels, and B. Mi. "Simulation and Measurement of Ultrasonic Waves in Elastic Plates using Laser Vibrometry." AIP Conference Proceedings, edited by Donald O. Thompson, and Dale E. Chimenti, Vol. 760, no. 1, pp. 172-179, 2005.

[3.14] A. Zak, M. Radzienski, M. Krawczuk, and W. Ostachowicz, "Damage Detection Strategies based on Propagation of Guided Elastic Waves," Smart Materials and Structures, vol. 21, p. $035024,2012$.

[3.15] P. Kudela, M. Radzienski, and W. Ostachowicz, "Identification of Cracks in Thin-walled Structures by means of Wavenumber Filtering," Mechanical Systems and Signal Processing, vol. 50, pp. 456-466, 2015. 
[3.16] B. Park, Y. K. An, and H. Sohn, "Visualization of Hidden Delamination and Debondiong in Composites through Noncontact Laser Ultrasonic Scanning," Composites Science and Technology, vol. 100, pp. 10-18, 2014.

[3.17] C. C. Chia, J.-R. Lee, C.-Y. Park, and H.-M. Jeong, "Laser Ultrasonic Anomalous Wave Propagation Imaging Method with Adjacent Wave Subtraction: Application to Actual Damages in Composite Wing," Optics \& Laser Technology, vol. 44, pp. 428-440, 2012.

[3.18] M. D. Rogge and C. Leckey, "Characterization of Impact Damage in Composite Laminates using Guided Wavefield Imaging and Local Wavenumber Domain Analysis," Ultrasonics, vol. 53, no. 7, pp.1217-1226, 2013.

[3.19] Z. Tian, L. Yu, and C. Leckey, "Delamination Detection and Quantification on Laminated Composite Structures with Lamb Waves and Wavenumber Analysis," Journal of Intelligent Mterial Systems and Structures, vol. 26, no. 13, pp. 1723-1738, 2015.

[3.20] R. Zhu, G. L. Huang, and F. G. Yuan, "Fast Damage Imaging Using the Time-Reversal Technique in the Frequency-Wavenumber Domain," Smart Materials and Structures, vol. 22, no. 7, p. $075028,2013$.

[3.21] M. S. Harb and F. G. Yuan, "Damage Imaging using Non-contact Air-coupled Transducer/Laser Doppler Vibrometer System,” Structural Health Monitoring, vol. 15, no. 2, pp. 193-203, 2016.

[3.22] M. S. Harb and F. G. Yuan, "Barely Visible Impact Damage Imaging using Non-contact Air-coupled Transducer/Lasr Doppler Vibrometer System," Structural Health Monitoring, $1475921716678921,2016$. 
[3.23] J. He and F. G. Yuan, "A Quantitative Damage Imaging Technique based on Enhanced CCRTM for Composite Plates using 2D Scan," Smart Materials and Structures, vol. 25, no. 10, 105022, 2016.

[3.24] J. Achenbach, Reciprocity in Elastodynamics, Cambridge University Press, 2003.

[3.25] F. A. Leone, D. Girolamo, and C. G. Dávila, "Progressive Damage Analysis of Bonded Composite Joints," NASA/TM-2012-217790, Hampton, VA, 2012.

[3.26] D. N. Alleyne and P. Cawley, "The Interaction of Lamb Waves with Defects," IEEE Transactions on Ultrasonics, Ferroelectrics, and Frequency Control, vol. 39, no. 3, pp. 381$397,1992$.

[3.27] Z. Su, L. Ye, and Y. Lu, "Guided Lamb Waves for Identification of Damage in Composite Structures: a Review," Journal of Sound and Vibration, vol. 295, no. 3, pp. 753-780, 2006.

[3.28] Y. Bar-Cohen, A. K. Mal, and Z. Chang, "Characterization of Defects in Composite Material Using Rapidly Acquired Leaky Lamb Wave (LLW) Dispersion Data," Proceedings of the 7th European Conference on Non-Destructive Testing, Copenhagen, Denmark, pp. 24-26, 1998. 


\section{CHAPTER 4 Damage Imaging in a Stiffened Curved Composite Sandwich Panel with Wavenumber Index via Riesz Transform}

\subsection{Introduction}

Carbon fiber reinforced polymer (CFRP) composite materials have been widely used in lightweight structures due to its high strength/weight and stiffness/weight ratio properties. Composite sandwich panels have been used extensively in stiffness-critical aircraft secondary components for improved structural efficiency and reduced maintenance cost. They consist of continuous fiber reinforced plastic face-sheets separated by low-density cores. The lightweight core, usually honeycomb or foam, is typically much thicker and much lighter than the face-sheets, with the aim of increasing the bending stiffness, the damage tolerance, the thermal resistance, the resistance to vibrations and noise reduction. However its geometric complexity increases the difficulty in damage detection due to various complex internal failure modes [4.1][4.2]. Unavoidable impacts from bird strikes or hail impacts occur to aircrafts during in-service operation, and impact accidents does happen from regular inspection or maintenance leading to unexpected damages. Unlikely aluminum or steel structures, these impacts introduce delamination and debonding beneath surfaces, or even crush for the core material that can deteriorate the residual strength significantly for composite structures [4.3]; moreover, they are barely visible for regular visual inspection, leading to potential safety issues and higher risk. Even though ultrasonic scan or X-ray computed tomography (CT) scan is capable of detecting barely-visible impact damage (BVID) in composites, the scanning is time-consuming and the techniques may require disassembly of the interrogated objectives [4.4]. Therefore, detection techniques based on guided wave have been of interest for years to detect, locate and identify these damages since guided wave carries abundant information by interacting with boundaries and geometric/material 
discontinuities during propagation without significant dissipation [4.5]. BVIDs in composite structures create discontinuity in mechanical properties which generates localized new wavenumber, wave reflection/refraction and sometimes generates local resonant vibrations, while guided waves propagates through the damage area.

Basically the more sensor/actuator used to collect/excite the wave signal, the more detailed damage information can be derived. One or very sparse sensors/actuators can be only capable of detecting the existence of damages by comparing the wave signals between pristine condition and damaged condition [4.6]. Once the amount of sensor/actuator increases, the damages can be further located; phased array (or beamforming) configuration is commonly used recently for damage localization than distributed sparse sensor/actuator [4.7]. When the amount of the sampling point is sufficiently large enough to meet the Nyquist criterion, the wave signal measured at each point can be reconstructed as a wavefield. The detailed shape and location of the damage can be visualized by analyzing the wavefield with damage imaging techniques. Even though several damage imaging methods have been suggested lately for damage visualization based on guided wavefield, they all possess their disadvantages and limitations. In this paper, a new damage imaging condition was developed to detect and visualize a BVID at the vicinity of a stiffener on a stiffened curved composite panel. In practical scenario, most techniques face difficulties for damages located near other damages, boundaries, stiffeners or on a geometrically complex surface [4.8][4.9]. The developed technique successfully visualized the damage with accuracy compared to the reference ultrasonic C-scan results, and it shows good consistency for different locations of excitation sources.

A root mean square (RMS)-based damage visualization method for full wavefield data was proposed by Ruzzene et al. [4.10]. The method is to simply evaluate the square root of the averaged 
signal energy (wave signal squared) in a time interval at every location of the wavefield and to form a corresponding map. This method is based on a premise that waves, either propagating or evanescent, scattered from the damages as a secondary source lead to wave energy re-distribution. Thus calculating RMS (or broadly mean signal energy) for every location may provide useful information about location of the damage. The numerical and experimental studies shown in [10] the scattered signal energy from a sizable damage (or scatterer) away from the piezo excitation location in flat isotropic plates can be visibly seen from the map, yet the location for piezoelectric wafer exciting the initial energy source is always highlighted in the map. This method was remedied by intentionally downplaying the signal energy at early times of the time interval through a weighted RMS (e.g., Zak et al. [4.11]).

A cumulative standing wave energy (CSWE) method has been proposed by Dr. Sohn's group (Sohn et al. [4.12], An et al. [4.13] and Park et al. [4.14]) for visualizing the cracks in metallic plates, delaminations in a flat composite laminate, and debonding between skin and stiffener in a composite laminates. In these studies the wavefield data covers the region of the damage which is assumed to be known in priori. Wavefields are first transformed into frequency-wavenumber $(f-$ k) domain through three-dimensional (3-D) Fourier transform for wavenumber filtering proposed by Ruzzene [15]. Then the propagating waves can be deduced from the transformed wavefield via window functions in each four quadrant of the wavenumber plane. The standing wave energy can be extracted by subtracting all the propagating wave energies from the total wave energy, and all these wave energies are derived in the physical time-space domain through inverse 3-D Fourier transform. At last, the CSWE that highlights local resonance within the damage region for visualizing the damage in composites is to integrate the standing wave energy in a time interval. In summary, the RMS method is a simple time domain method, focusing on the damage-induced 
local high intensity in the wavefield to highlight the damaged region, and the local intensity is often masked in the area with large wavefield amplitude or in close proximity of the geometric features. Even though CSWE involves wavenumber filtering to enhance the effect of the local intensity of standing wave, they can be still obscured by the close proximity of the geometric feature.

Yuan and Harb [4.16][4.17] introduced a zero-lag cross-correlation (ZLCC) damage imaging technique for visualization of delamination damage on wavefields. The incident and reflected waves are first separated in the $f-k$ domain followed by obtaining the two wavefields in the frequency domain via 2-D spatial Fourier transform. The two wavefields are cross-correlated with zero time-lag to generate damage image by summing all the frequency contents. Girolamo et al. [4.18] further redefined the forward and backward propagating waves in improved twodimensional wavenumber filtering process and employed ZLCC in frequency domain, to highlight the local resonance within the damage region in a honeycomb composite panel. The image formed from ZLCC in frequency domain shows better damage imaging result with less influence from ambient noise and is capable of extracting the hidden resonant phenomena at higher local resonant frequencies. However, the correlation value of ZLCC still relies on the intensity of the incident and reflected waves. This implies that the correlation value directly depends on how the reflected waves from the damage have been attenuated either by the far distance from the actuation location or by other geometric or material discontinuity, not associated with damage severity.

There are several other damage imaging algorithms by taking advantage of localized wavenumber value to highlight the discontinuity in mechanical properties. Rogge and Lecky [4.19] introduced a wavenumber domain analysis via a mode separation technique followed by a short-windowed Fourier transform (SFT) in space domain to obtain local wavenumber from the wavefield for 
characterization of impact damages in a composite plate. The local wavenumber value is defined as the maximum wavenumber value within the short-windowed region and the local wavenumber is defined at the center of the window. The proposed wavenumber domain analysis provides good agreement with theoretical prediction of spatial window selection, and the damage imaging result shows the capability to characterize the depth of the delamination in composite laminate. Then the technique was further enhanced by Juarez and Lecky [4.20] to multi-frequency local wavenumber analysis along with a ply correlation technique to quantify the impact damage. With similar concept of using localized wavenumber to highlight the damage region, Mesnil et al. [4.21] proposed an instantaneous wavenumber estimation method for damage quantification, by employing one-dimensional (1-D) spatial Hilbert transform (HT) in the wavefield for obtaining instantaneous wavenumber. The two-dimensional instantaneous wavenumber generalization was then defined as the square root of the sum of instantaneous wavenumber squared in both two directions. Flynn et al. [4.22] introduced a damage imaging condition using local wavenumber estimation in the frequency-wavenumber domain to image both the structural features and defects. A wave mode separation technique was first applied onto the wavefield signal to isolate a narrowband and single mode wave signal with its center frequency and center wavenumber, and local wavenumber were defined as the highest magnitude of the space-wavenumber representation for each point. The space-wavenumber representation at given center wavenumber is calculated by the sum of three-dimensional monogenic envelopes of filtered wavefield signal at center wavenumber within the interested sampling period. But simply operating HT in two directions separately is not the genuine two-dimensional generalization, and both HT and SFT techniques require detailed material characteristic for wave mode separation and filtering for obtaining the 
local wavenumber. For structures with unknown material and composite layup, the real wavenumber value is often not available to be derived.

In this paper, a new guided wave-based damage visualization method employing Riesz transform (RT) as an image processing technique is developed on a stiffened curved composite panel. The developed damage imaging technique, named wavenumber index (WI), takes advantage of the abundant information contained in monogenic signals representation of the guided wavefield. The monogenic signal derived by RT, the genuine two-dimensional generalized HT in space domain, is to obtain instantaneous orientation, phase and amplitude of the reconstructed guided wavefield. The instantaneous wave energy is calculated from instantaneous amplitude, and the instantaneous wave vector is obtained by taking derivative of the instantaneous phase with respect to two coordinates in space domain. The magnitude of the instantaneous wave vector derived via RT indicates the instantaneous wavenumber value pointing the propagation direction at each point. The derivation of wavenumber vector involves no time-frequency transformation and requires no filtering in frequency-wavenumber domain. Since the wavenumber value is difficult to be specifically derived for structures with unknown material information, the WI is defined by integration of instantaneous wavevector throughout the sampling interval to highlight the wavenumber change due to discontinuity of material properties at the damaged area. The phase information from monogenic signal reserves detailed information for all scattered and reflected wavefield with very small intensity, so that the developed WI method is capable of dealing with structures with complex geometry.

The panel used in this paper was preliminarily impacted and examined in NASA Langley Research Center as described in Section 4.2. The experimental setup of the integrated non-contact scanning system consisted of Nd:YAG pulse laser and LDV is illustrated in Section 4.3. The derivation of 
monogenic wavefield and the instantaneous wave vector is shown in Section 4.4. The phase denoising procedure and the developed damage imaging condition is described in Section 4.5. The damage imaging results and comparison of ZLCC and WI are given in Section 4.7. The conclusion and the summary are given in the last part, Section 4.7.

\subsection{Preliminary Examination}

The stiffened curved composite sandwich panel used in this paper is a section of aileron from a Boeing C-17 Globemaster III aircraft. The curved geometry and material/geometry discontinuities increase the difficulty for analyzing the guided wavefield. In order to create the real impact damage instead of an artificial damage, the panel was impacted at NASA Langley Research Center with a $\phi 25.4 \mathrm{~mm}$ hemispherical impactor and the impact energy of $15 J(11 \mathrm{ft}-\mathrm{lbs})$. The panel geometry of the composite foam panel and the impact location is shown in Figure 3.1(a), and the experimental setup of the ball-drop impactor is shown in Figure 3.1(b). A location was intentionally impacted in close proximity to the T-joint internal stiffener, which is bonded to the back surface of the panel. The joint region, stiffener and damage location is illustrated as a side view of cross-section of the scanning area as Figure 3.1(c). The T-joint stiffeners are also made of composites, and the layup is unknown. The jointed region is around $42 \mathrm{~mm}$ wide with the $3 \mathrm{~mm}$ thick stiffener in the center and the impact damage is located only about $7 \mathrm{~mm}$ from the edge of the joint. Even though the BVID created on the face-sheet only shows as a small superficial dent on the surface, it may reduce the strength of the composite structure significantly by $20 \% \sim 30 \%$ [4.23]. 


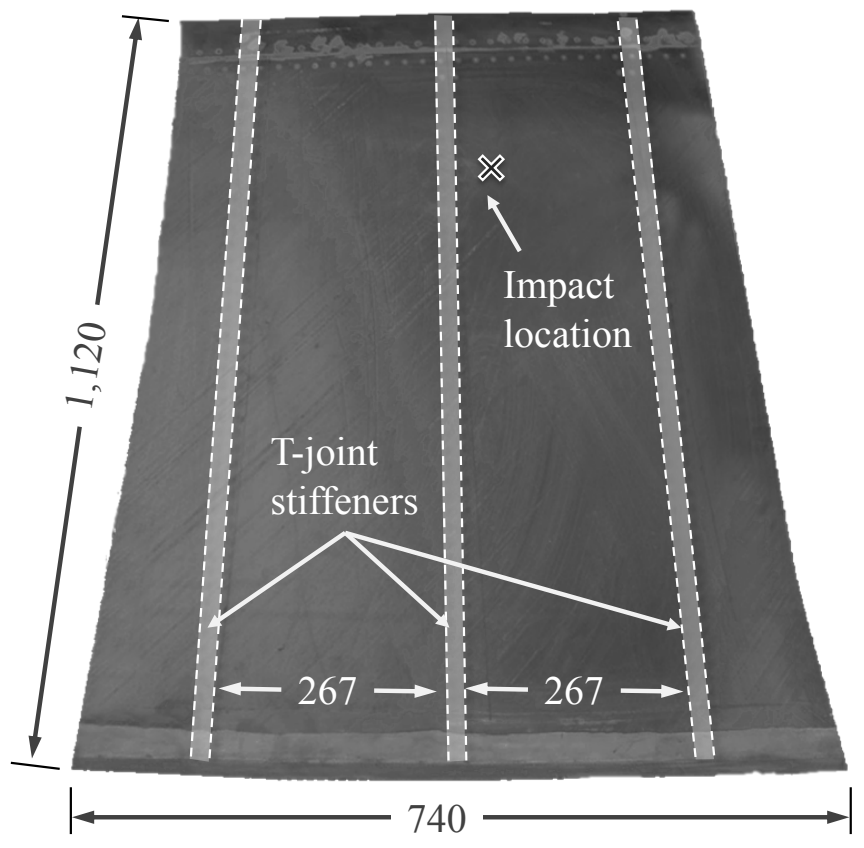

(unit: $m m$ )

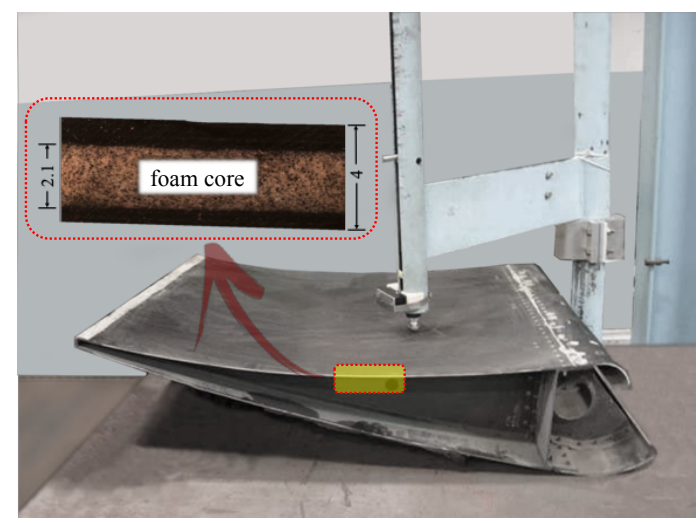

(b)

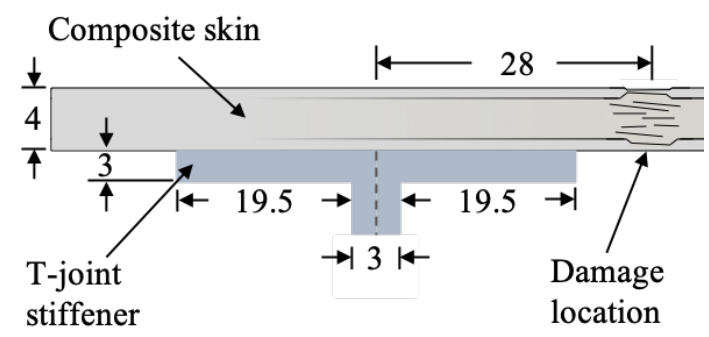

(a)

(c)

Figure 3.1.(a) The geometry of the stiffened curved composite foam panel (not-to-scale),

(b) The illustration of ball-drop impact test, (c) The illustration for the cross section of the impact damage region close to the stiffener (not-to-scale).

For comparison purpose, the impact damage was imaged by ultrasonic ultrasonic C-scan as a baseline. The ultrasonic C-scan system consists of an Olympus submersion transducer and a Panametrics Model 5055 PR-101 pulser receiver operating at $10 \mathrm{MHz}$. The sampling resolution was $0.254 \mathrm{~mm}$, and the sampling area was a $38 \mathrm{~mm} \times 38 \mathrm{~mm}$ square covering the damage area, generating scan images with $151 \times 151$-pixel resolution. The images were interpolated in MATLAB to produce higher-definition images. The ultrasonic C-scan system setup is shown in Figure 3.2(a) and the final result shown in Figure 3.2(b). A RMS algorithm embedded in the C-scan system was 
employed to generate the damage image throughout the thickness to highlight all of the delaminations underneath the face-sheet as shown in Figure 3.12 in the Appendix.

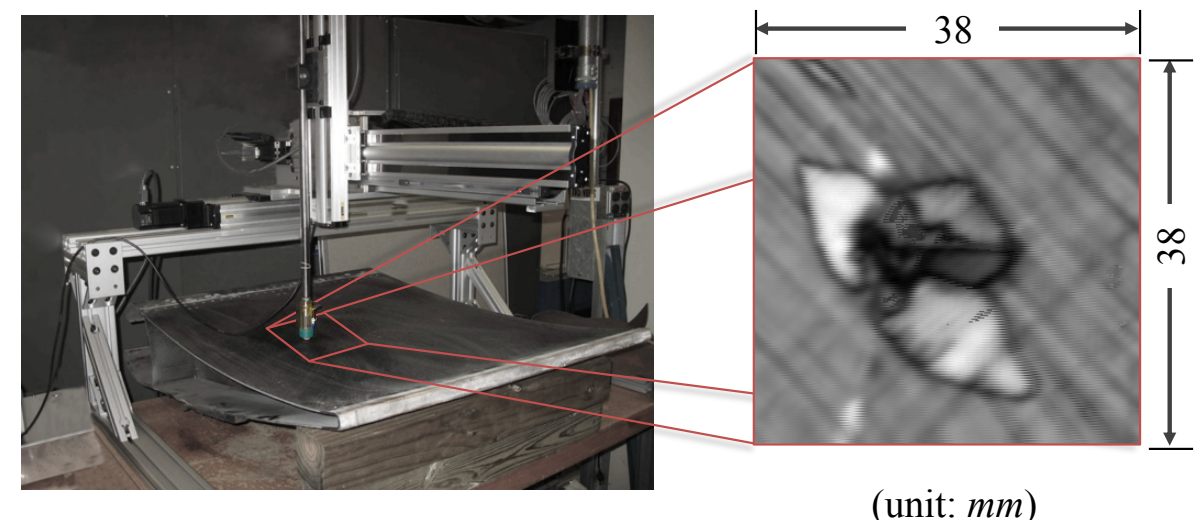

Figure 3.2. The ultrasonic C-scan experimental setup and the result for the impact damage.

\subsection{Experimental Setup}

An impact damage induced at a location far away from the stiffener on the same composite sandwich panel was initially investigated by He and Yuan [4.24]. Several imaging condition methods were used and compared using controlled ultrasonic waves with piezoelectric (PZT) as actuators and laser Doppler vibrometer (LDV) as sensors. A signal received in frequency selection test was first conducted on the panel, and the excitation signal center frequency was selected as 60 $\mathrm{kHz}$ for the relatively large and dominant $\mathrm{A}_{0}$ wave mode and avoiding complex scattering patterns. The impact damage investigated in this research was initially studied by Chang and Yuan [4.25] using the automated PZT/LDV scanning system with a five-cycle Hanning-windowed toneburst signal at center frequency $60 \mathrm{kHz}$. In this paper, the broadband ultrasonic guided waves were excited by a Nd:YAG Q-switched pulse laser, and the out-of-plane velocity was collected pointby-point by a LDV (Polytec OFV-505) mounted on an programmable translational stage. The LDV location was fixed during the scanning, and the pulse laser beam was steered by a calibrated 
galvomirror to excite the ultrasonic guided wave on the designed grid precisely within the interrogation region. Due to the Betti's reciprocity theorem extended to the linear elastodynamic case [4.26], the reconstructed wavefield is equivalently generated from the LDV location and then propagates towards the interrogation region. Each signal was measured repeatedly thirty times average to minimize the incoherent noise, thus improving signal-to-noise ratio, and the experiments were performed separately from four LDV locations outside the scanned region. The wavefield image was then reconstructed from the three-dimensional data matrix; namely in-plane coordinate and time. The scanning system setup is illustrated as Figure 3.3. The PC controls galvomirror to point the pulse laser beam on the grid precisely and the translational stage to locate the laser beam of the LDV at the sensing location. The pulse laser is triggered by the simple Transistor-Transistor Logic (TTL) signal sent from the PC, and another TTL signal is sent from pulse laser to LDV for synchronization.

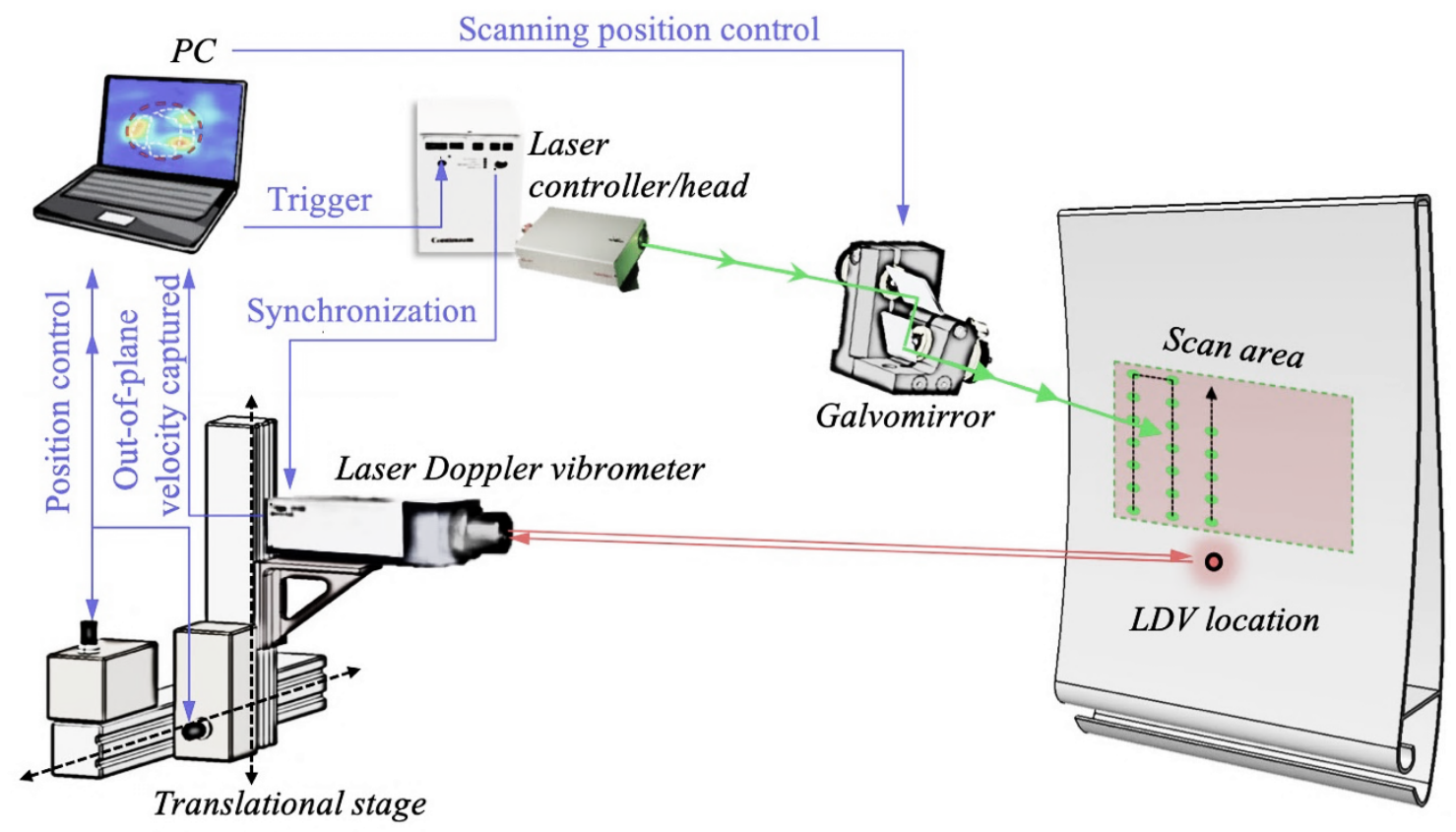

Figure 3.3. A schematic of laser scanning system for imaging the barely visible impact damage 


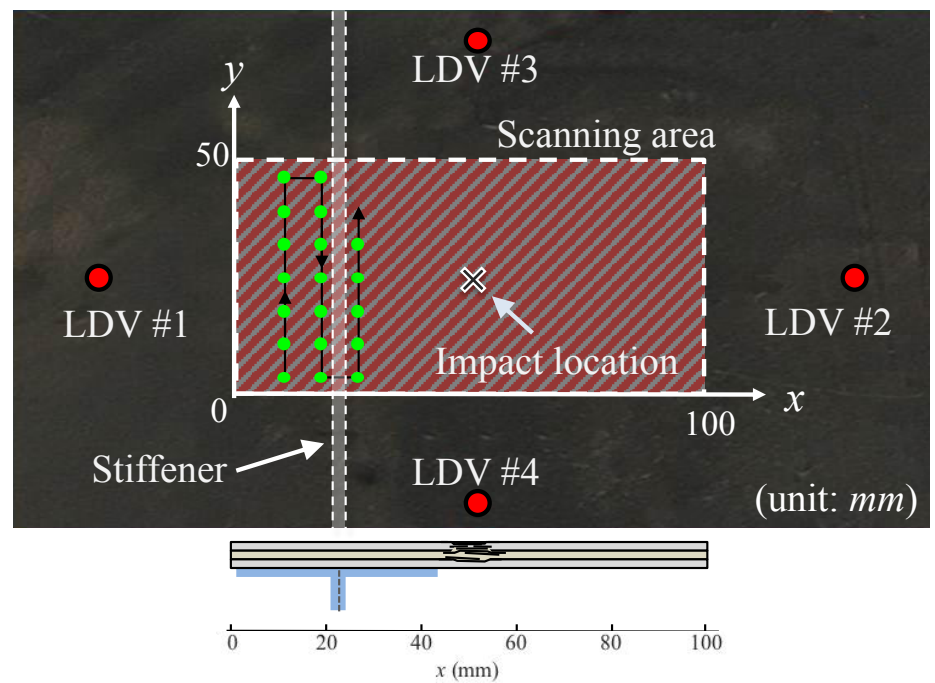

Figure 3.4. The illustration of LDV locations, scanning area and the crosssection of the panel

The pulse laser used to excite the ultrasonic signal was an Nd:YAG Q-switched laser producing $532 \mathrm{~nm}$ wavelength light. The repetition rate of pulse laser is $15 \mathrm{~Hz}$, each of which has energy of $16 \mathrm{~mJ}$ and pulse-width of 5-7 nsec. The pulse laser beam diameter was measured to be about 3.5 $m m$ on the panel. The Nd:YAG pulse laser was raster scanned on the panel surface by an optical scanner using a set of galvano-mirrors having a repeatability of $8 \mu \mathrm{rad}$ and positioned at about 500 $\mathrm{mm}$ normal from the panel. To examine the characteristics of the excitation signal generated by the pulse laser, it is convenient to measure the signal using LDV right next to the location, roughly $1-2 \mathrm{~mm}$ as shown in Figure 3.5 since the wavefield is captured from the LDV. Figure 3.5(a) shows the signal in time domain; the Figure 3.5(b) shows its magnitude in frequency domain. The LDV was placed about $0.9 \mathrm{~m}$ away from the test article. The LDV decoder for the velocity measurement was set to VD-09 $10 \mathrm{~mm} / \mathrm{s} / \mathrm{V}$ and the sampling frequency was $2.56 \mathrm{MHz}$. It yielded the maximum measurable frequency at $250 \mathrm{kHz}$ and the maximum sensitivity at frequencies around $100 \mathrm{kHz}$, as shown in Figure 3.5(b). For better signal-to-noise ratio (SNR), the measured data was averaged 30 times, and the reflective tapes were used at each LDV scanning location. The total sampling point was $3321(41 \times 81)$ with spacing of $1.25 \mathrm{~mm}$, so that the scanning area was a $50 \mathrm{~mm}$ 
$\times 100 \mathrm{~mm}$ rectangle, and the stiffener was covered in the scanning area. The $1.25 \mathrm{~mm}$ spacing provided the spatial sampling frequency at $8001 / \mathrm{m}$, and the measuring range could be up to 400 $1 / m$ in wavenumber value, according to Nyquist sampling theorem. The wavefield image was then reconstructed by interpolating the measured data with cubic spline. The wavenumber maps of the wavefield propagating in four directions then were obtained by taking Fourier transform in space domain, as shown in Figure 3.6. Since the excitation was a wideband signal, the wavenumber values were located in a relatively large region than a signal point, and the values were roughly between $401 / \mathrm{m}$ to $1001 / \mathrm{m}$. It indicated that the sampling spacing of $1.25 \mathrm{~mm}$ is more than sufficient for the wavefield measurement and reconstruction.

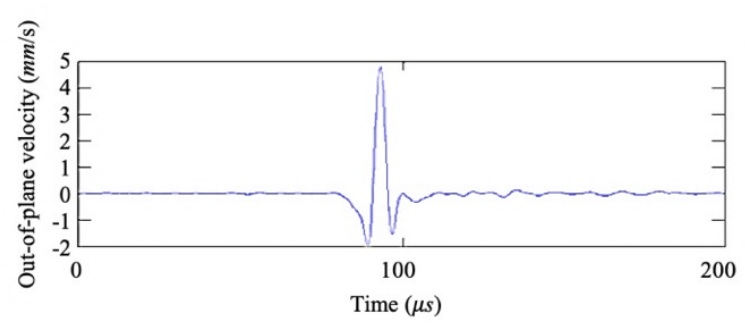

(a) Time domain

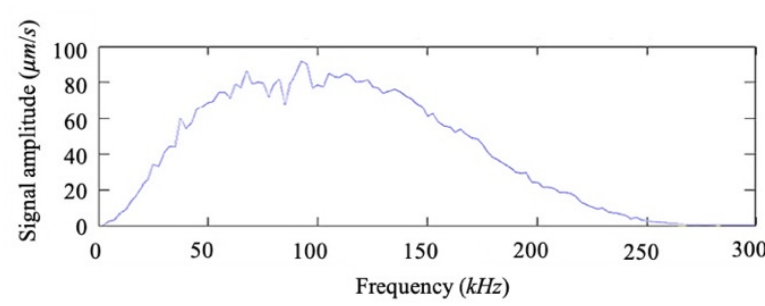

(b) Frequency domain

Figure 3.5. Excitation signal in the honeycomb composite panel generated by the pulse laser was measured by a nearby LDV. 


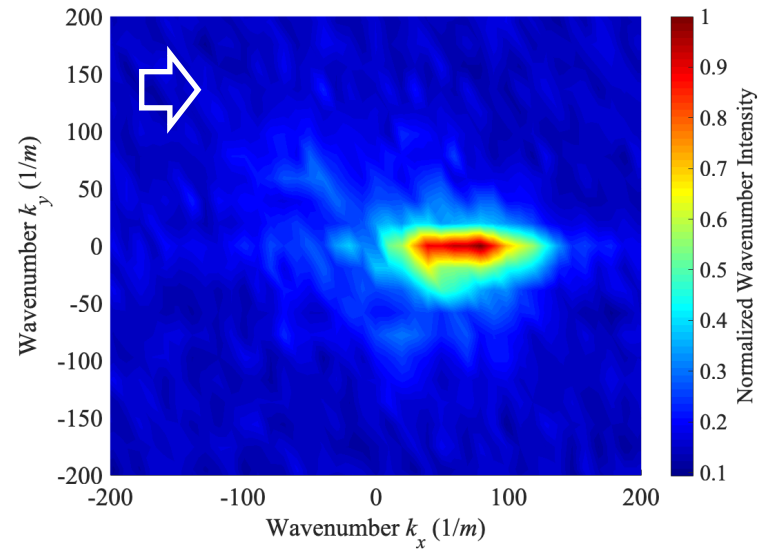

(a)

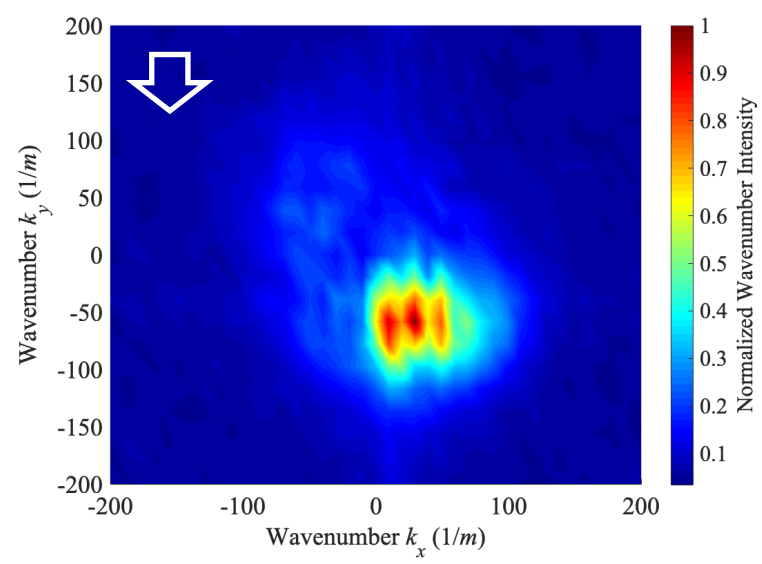

(c)

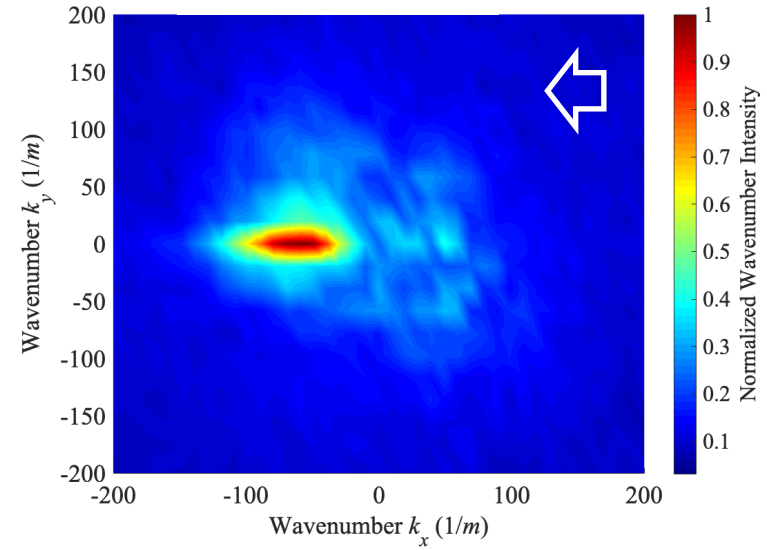

(b)

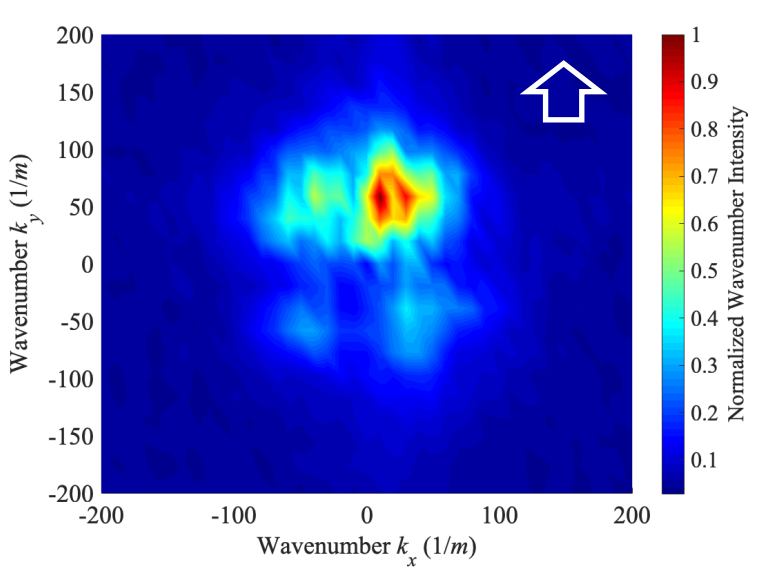

(d)

Figure 3.6. The wavenumber map of the wavefield excited from four locations shown in Figure

3.4. The arrow indicates the wave propagating directed toward the damage region.

(a) \#1, rightward, (b) \#2, leftward, (c) \#3, downward and (d) \#4, upward.

The BVID through the thickness of the panel disrupts the wave progression, where some portion of the wave energy reflects back from the boundary of the damage and the remaining portion refracts into the damaged region. While within the small delaminated region, a superposition of waves propagating in opposite (forward and backward) directions generates momentary standing waves in all directions trapped within this region. This portion of wave energy trapped in the damaged region is then called as standing wave energy. The guided wavefield is disturbed when 
it encounter the damage area with reduced stiffness and these anomalies can be further measured and extracted from damage imaging condition. The snapshots of wavefields in scan \#1 and \#2 at $t=160 \mu s$, and scan $\# 3$ and $\# 4$ at $t=140 \mu s$ are shown in Figure 3.7, the direction of the wave progression is also denoted as the arrow at the upper left corner in each figure. Figure 3.7(a) shows the snapshot of the wavefield for LDV location \#1 with wave propagating toward the right, Figure 3.7(b) for impact LDV location \#2 with wave propagating toward the left, Figure 3.7(c) for LDV location \#3 with wave propagating downward, and Figure 3.7(d) for LDV location \#4 with wave propagating upward. The disturbance of the wavefield induced by the discontinuity at the damaged area can be clearly seen in all four snapshots; however, it is less clear for Figure 3.7(a) since wave energy attenuated significantly while the wave passes through the stiffener. The joint area is marked as a shaded area on the left of the wavefield image, and the stiffener is marked as the light area with dashed lines. The impact damage image is marked with red dashed line according to the ultrasonic C-scan result previously shown in Figure 3.2(b). 


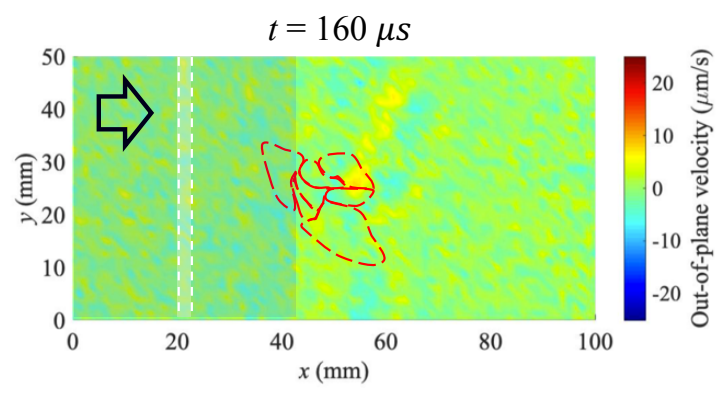

(a)

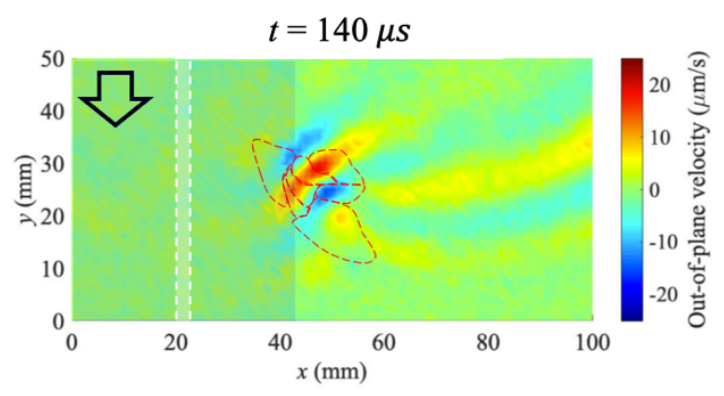

(c)

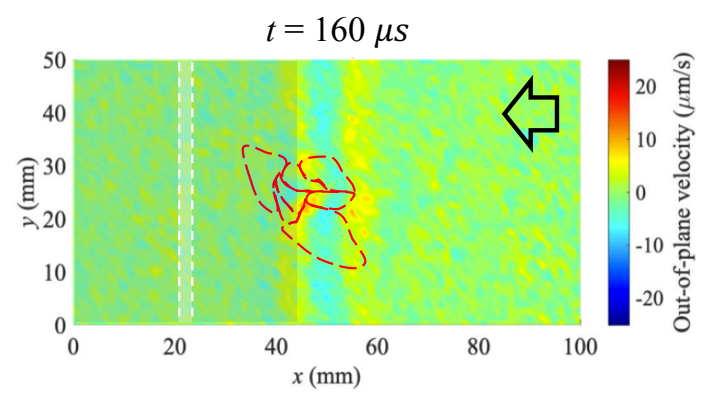

(b)

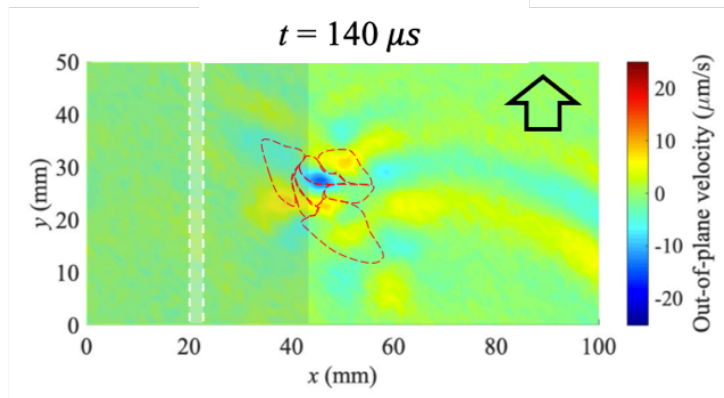

(d)

Figure 3.7. The snapshot of the wavefield "video" for (a) \#1, rightward, (b) \#2, leftward, (c) \#3, downward and (d) \#4, upward.

\subsection{Monogenic Wavefield and Instantaneous Wave Vector}

The complex-valued representation of any real signal, which is also named as the analytic signal representation, is commonly used as an alternative representation for simple mathematical representation and computational efficiency; however, every real signal requires an appropriately derived imaginary part to construct the complex representation without loss of signal energy and phase distortion. Hilbert transform (HT) is a well-known tool to compute the imaginary parts of real-valued signals in order to generate its analytic signal. HT provides local amplitude and phase information through generating analytic signal along any orientation of interest. HT is defined as 
the convolution of $1 / \pi t$ and the real function $f(t)$, which in frequency domain is the product of isgn $\omega$ and Fourier transformed function $\tilde{f}(\omega)$, as shown in Eq.(3.1).

$$
\mathcal{H}[f(t)]=\frac{1}{\pi t} \otimes f(t) \stackrel{F F T}{\longrightarrow} i \operatorname{sgn} \omega \cdot \tilde{f}(\omega)
$$

where $\operatorname{sgn}(\omega)=\left\{\begin{array}{cl}1 & , \text { for } \omega>0 \\ 0 & , \text { for } \omega=0 \\ -1 & , \text { for } \omega<0\end{array}, \mathcal{H}(\cdot)\right.$ is Hilbert transform operator, $\otimes$ denotes convolution operator, and $\tilde{f}(\omega)$ is the fast Fourier transform (FFT) of the function $f(t)$. The HT of the $f(t)$ is the purely imaginary part $f_{H}(t)$ of the analytic signal $f_{+}(t)$, where $f_{+}(t)=f(t)+i f_{H}(t)$, and $f_{H}(t)$ is also called the quadrature pair of the real signal $f(t)$. The analytic signal $f_{+}(t)$ can be expressed in a polar form with amplitude $A(t)$ and phase $\phi(t)$ as shown in Eq. (3.2), where $A(t)=\sqrt{|f(t)|^{2}+\left|f_{H}(t)\right|^{2}}$, $\phi(t)=\tan ^{-1}\left[f_{H}(t) / f(t)\right]$. The instantaneous frequency $\omega(t)$ can be further calculated as the derivative of the instantaneous phase $\phi(t)$ as the expression $\omega(t)=d \phi(t) / d t$.

$$
f_{+}(t) \equiv A(t) e^{i \phi(t)}
$$

HT has been considered as a robust time-frequency analysis tool, and it can be further employed for space-wavenumber analysis when HT is operated in space domain to obtain the local instantaneous spatial phase and local instantaneous wavenumber (IW). A damage imaging condition using IW was developed and applied to highlight the local wavenumber induced by the discontinuity of material properties around the damage area. Since HT is only capable of dealing with one-dimensional (1-D) signal, the IW was calculated by employing HT and taking derivative of the instantaneous spatial phase with respect to two orientations separately [4.21]. However, the generalization of two-dimensional (2-D) IW obtained from the spatial phase by simply calculating 
HT twice at each orientation is not isotropic and lacks the invariance-equivariance property for local phase and amplitude [4.27].

Given a displacement function of the plane wavefield $w(x, y, t)$ at any time instant $t$, it can be represented as the real part of the complex-valued signal by defining the phase of the signal $\phi(x, y, t)=k_{x} x+k_{y} y-\omega t=\mathbf{k x}-\omega t$, where $k_{x}, k_{y}$ is the wavenumber along $x, y$ direction respectively, $\mathbf{k}=\left[k_{x}, k_{y}\right]^{\prime}$ is the direction of wave propagation and $\omega$ is the temporal frequency as shown below.

$$
w(x, y, t)=\operatorname{Re}\left[e^{i\left(k_{x} x+k_{y} y-\omega t\right)}\right]=\cos \left(k_{x} x+k_{y} y-\omega t\right)=\cos \phi
$$

At a given time instant $t$, the instantaneous wavefield can be considered as an image snapshot. Since the wavefield is 2-D signal, two imaginary parts are needed to construct the quadrature image for obtaining the complex-valued wavefield. Therefore, Riesz transform (RT) and the monogenic signal of Felsberg and Sommer [4.28], proven to be appropriate 2-D generalization of HT and analytic signal, are introduced to derive the instantaneous wavenumber of the wavefield. For the displacement $w(x, y, t)$ at a given time instant $t$, the definition of RT is given by

$$
\mathcal{R}[w(x, y, t)]=-\frac{\mathbf{x}}{2 \pi|\mathbf{x}|^{3}} \otimes w(x, y, t) \stackrel{\text { 2-D FFT }}{\longrightarrow} i \frac{\mathbf{k}}{|\mathbf{k}|} \tilde{w}\left(k_{x}, k_{y}, t\right)
$$

where $\mathcal{R}(\cdot)$ denotes RT operator, $\otimes$ denotes convolution operator, $\tilde{w}\left(k_{x}, k_{y}, t\right)$ is the spatially 2 -D

FFT of the wavefield at time $t, \mathbf{x}=[x, y]^{T}$ is the local spatial coordinate, and $\mathbf{k}=\left[k_{x}, k_{y}\right]^{T}$ is the local coordinate in wavenumber domain. Similarly, the RT can be considered as a product of the spatially Fourier transformed signal and the RT transfer function in the wavenumber domain. The two imaginary parts for constructing the quadrature image are then: 


$$
\begin{aligned}
& \tilde{\hat{w}}_{|x|}\left(k_{x}, k_{y}, t\right)= \begin{cases}i \frac{k_{x}}{|\mathbf{k}|} \tilde{w}\left(k_{x}, k_{y}, t\right), & \mathbf{k} \neq 0 \\
0, & \mathbf{k}=0\end{cases} \\
& \tilde{\hat{\hat{w}}}_{|y|}\left(k_{x}, k_{y}, t\right)= \begin{cases}i \frac{k_{y}}{|\mathbf{k}|} \tilde{w}\left(k_{x}, k_{y}, t\right), & \mathbf{k} \neq 0 \\
0, & \mathbf{k}=0\end{cases}
\end{aligned}
$$

where $\tilde{\hat{w}}_{|x|}\left(k_{x}, k_{y}, t\right)$ and $\tilde{\hat{w}}_{|y|}\left(k_{x}, k_{y}, t\right)$ are the RT of the real wavefield along $x$-axis and $y$-axis in wavenumber domain, and the two imaginary parts to construct the quadrature image and the monogenic wavefield are the inverse FFT of $\tilde{\hat{w}}_{|x|}\left(k_{x}, k_{y}, t\right)$ and $\tilde{\hat{w}}_{|x|}\left(k_{x}, k_{y}, t\right)$. The RT operator $i \frac{k_{x}}{|\mathbf{k}|}$ and $i \frac{k_{y}}{|\mathbf{k}|}$ in wavenumber domain is illustrated in Figure 3.8(a) and Figure 3.8(b) respectively.

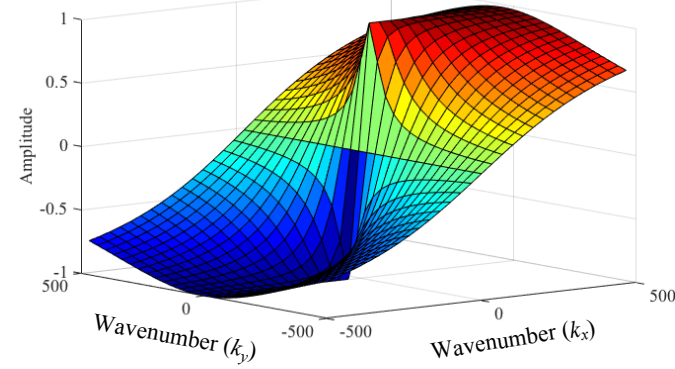

(a)

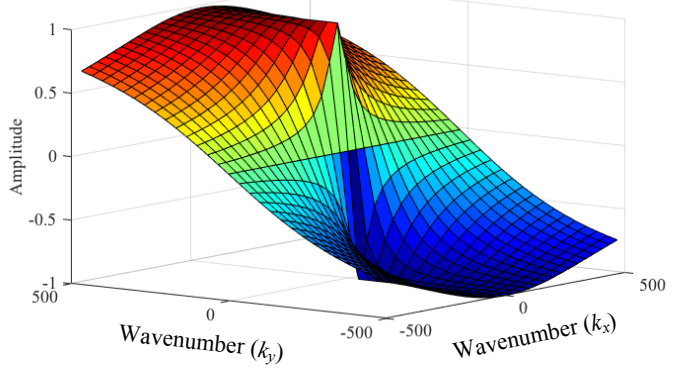

(b)

Figure 3.8. Illustrastion of Riesz transform operator in wavenumber domain along (a) $x$-axis as shown in Eq. (3.5), (b) $y$-axis as shown in Eq. (3.6).

The Riesz transformed wavefield signal can be represented as a Riesz triple vector [4.27] for the construction of monogenic signal as: 


$$
w_{+}(x, y, t)=\left[w(x, y, t), \hat{w}_{|x|}(x, y, t), \hat{w}_{|y|}(x, y, t)\right]^{T}
$$

where $w_{+}(x, y, t)$ is the monogenic signal of the original wavefield, and $\hat{w}_{|x|}, \hat{w}_{|y|}$ are the RT along $x$ and $y$ axis respectively. Also, from the monogenic signal, the instantaneous wave energy is defined as

$$
\mathrm{E}(x, y, t)=|w(x, y, t)|^{2}+\left|\hat{w}_{|x|}(x, y, t)\right|^{2}+\left|\hat{w}_{|y|}(x, y, t)\right|^{2}
$$

And the local instantaneous amplitude which is also the envelope of the monogenic signal, is defined as

$$
\mathrm{A}(x, y, t)=\sqrt{|w(x, y, t)|^{2}+\left|w_{R}(x, y, t)\right|^{2}}
$$

where $w_{R}(x, y, t)=\sqrt{\left|\hat{w}_{|x|}(x, y, t)\right|^{2}+\left|\hat{w}_{|y|}(x, y, t)\right|^{2}}$ is the RT steered to the dominant orientation. The spatial phase $\phi(x, y, t)$ and the Riesz orientation $\theta(x, y, t)$ at any instant can be calculated from the Riesz triple vector as shown in Eq. (3.10) and (3.11), and the illustration for the concept of orientation, phase direction and the wavefront is shown in Figure 3.9.

$$
\begin{aligned}
& \phi(x, y, t)=\tan ^{-1}\left(\frac{w_{R}(x, y, t)}{w(x, y, t)}\right) \\
& \theta(x, y, t)=\tan ^{-1}\left(\frac{\hat{w}_{|y|}(x, y, t)}{\hat{w}_{|x|}(x, y, t)}\right)
\end{aligned}
$$




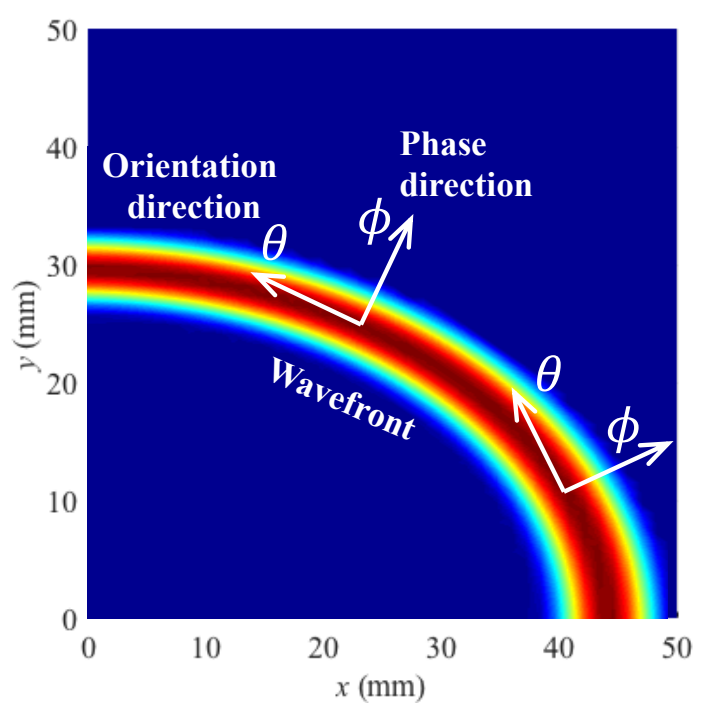

Figure 3.9. The illustration of wavefront, orientation and dominant phase direction as described in Eq. (3.10) and (3.11).

The spatial phase here is defined as the phase derived along the dominant direction normal to the wavefront, where the wavefield signals possess the maximum variation, which is the direction of phase velocity; the Riesz orientation is defined tangent to the wavefront, where the wave signal is least changing [4.28]. In short, at any given $x, y$ and $t$, the Riesz representation can be written in terms of the local amplitude $A$, the local dominant orientation $\theta$ and the local spatial phase $\phi$ as [4.29]:

$$
w=\mathrm{A} \cos \phi, \hat{w}_{|x|}=\mathrm{A} \sin \phi \cos \theta, \hat{w}_{|y|}=\mathrm{A} \sin \phi \sin \theta
$$

and

$$
w=A \cos \phi, w_{R}=\mathrm{A} \sin \phi
$$

The RT steered to its dominant orientation is the quadrature pair of the original wavefield signal at the given location and instant, and it can be applied as the imaginary part to generate the analytic 
signal as Eq. (3.8). Therefore, the steered RT can be considered as the HT along its dominant orientation.

$$
w_{+}=w+i w_{R}=\mathrm{A} \cos \phi+i \mathrm{~A} \sin \phi=\mathrm{A} e^{i \phi}
$$

The local spatial phase derived from RT is exactly the phase of the original wavefield $\phi(\mathbf{x}, t)=\mathbf{k x}-\omega t$. The instantaneous wave vector derived via Reisz transform is then defined as Eq. (3.15)

$$
\mathbf{k}=\nabla \phi(\mathbf{x}, t)=\left[\frac{\partial \phi}{\partial x}, \frac{\partial \phi}{\partial y}\right]^{T}
$$

The instantaneous wavenumber (IW) is then the magnitude of the instantaneous wave vector:

$$
\operatorname{IW}(x, y, t)=|\mathbf{k}|=\sqrt{\left(\frac{\partial \phi}{\partial x}\right)^{2}+\left(\frac{\partial \phi}{\partial y}\right)^{2}}
$$

The generalization of 2-D IW obtained by employing HT with respect to two orientations separately does not truly represent the real instantaneous wavenumber of the whole wavefield. RT is the generalized HT for 2-D signal processing, and it is used in this paper for computing the amplitude and the phase [4.28], giving the real instantaneous information. This generalized IW method effectively shows the discontinuity at the damage area; however, the technique increases not only sensitivity but also noise because the derivation of IW requires taking derivative of the phase which will amplify the effect of ambient noise. Therefore, a phase denoising technique is needed to eliminate the unwanted magnified noise, and the IW need to be integrated with consideration of wave energy for better damage imaging result. The phase denoising and the damage imaging condition: Wavenumber Index will be described in next Section. 


\subsection{Phase Denoising and Damage Imaging via Wavenumber}

The IW derived via Riesz transform provides detailed space-wavenumber information of the wavefield at any instant. It does not require complex wave mode separation and filtering in frequency-wavenumber domain for the operation, which is computationally efficient and robust. However the noise becomes non-negligible after extracting the phase from the analytic or monogenic signal, since the amplitude information has been eliminated. For improving the quality of the result, the amplitude information is again introduced to the IW as instantaneous wave energy (IWE) filter. The IWE is defined as the normalized wave energy, which is the ratio of wave energy of each point and the maximum energy among all points at the instant, as shown in Eq.(4.17). The signal with IWE below threshold is considered as noise, and the phases derived from noise signals are considered negligible. In this paper, the threshold is set at 3\% of the maximum instantaneous wave energy. With the introduction of the IWE, the influence of noise can be effectively suppressed for improving image quality. Furthermore, wavenumber index (WI) can be defined as the integration of the product of the determined IW value and weight IWE throughout the sampling interval, as shown in Eq.(4.17).

$$
\operatorname{IWE}\left(x, y, t_{0}\right)=\frac{\mathrm{E}^{2}\left(x, y, t_{0}\right)}{\mathrm{E}_{\text {Max }}^{2}\left(t_{0}\right)}
$$

Unfortunately, the noise of instantaneous phase will be enlarged significantly after taking derivative. A spatial amplitude-weighted Gaussian blur denoising method previously used in Riesz pyramid motion magnification [4.29] is applied for spatial smoothing of the derived instantaneous phase.

$$
\phi_{\text {Smooth }}=\frac{\mathrm{A} \phi \otimes K_{\sigma}}{\mathrm{A} \otimes K_{\sigma}}
$$


where $K_{\sigma}$ is the Gaussian blur kernel with standard deviation $\sigma$ and local coordinate $(x, y), \otimes$ denotes convolution operator, A is the instantaneous amplitude derived by RT, wand the Gaussian Kernel is known as

$$
K_{\sigma}(x, y)=\frac{1}{2 \pi \sigma^{2}} e^{-\frac{x^{2}+y^{2}}{\sigma^{2}}}
$$

In this paper, the Gaussian blur kernel is set at $\sigma=1$ and the filter size is 3 . The spatial smoothing effectively reduces the noise influence in the IW derivation, enhancing the quality of the damage imaging result. Since the IW derived via Riesz transform shows all the wavenumber values, of which the guided wave changes at the damage location with reduced stiffness, either less support or weaker bonding despite of the propagating direction. The damage area can then be visualized by integrating all the wavenumber values above the IWE threshold throughout the sampling time, so that the difference in wavenumber can be highlighted. The noise can be averaged out and the image quality can be enhanced after integration, but the integrated wavenumber cannot represent the real wavenumber at the location and the instant. Therefore, in this paper, the wavenumber index is shown in the normalized value, and then becomes:

$$
\overline{\mathrm{WI}}(x, y)=\frac{\mathrm{WI}(x, y)}{\operatorname{Max}[\mathrm{WI}(x, y)]}
$$

where $\operatorname{WI}(x, y)=\sum_{t=t_{i}}^{T} \operatorname{IW}(x, y, t)-\operatorname{Min}\left(\sum_{t=t_{i}}^{T} \operatorname{IW}(x, y, t)\right), t_{i}$ is the initial time instant of the interested segment of sampling, and $T$ is the total period. The WI yields a wavenumber map for damage imaging with good sensitivity, computational efficiency as well as accuracy. The damage identification result with WI is given and compared with the C-scan result and ZLCC result in the next Section. 


\subsection{Results and Comparison}

The stiffened curved composite sandwich panel was scanned from four different LDV locations, as shown in Figure 3.4. The ZLCC results shown in Figure 3.10 are generated and compared with results from the proposed WI shown in Figure 3.11. The joint area is marked as a white shaded area on the left of the wavefield image, and the stiffener is marked as the shaded area with white dotted line. The damage is marked as the red dashed line with the rectangular C-scan region marked as the red solid line. The results for the wavefield of LDV location \#1 with guided wave propagating toward right are shown Figure 3.10(a) and Figure 3.11(a), and the image quality of ZLCC is apparently poorer than others because majority part of the wave energy is blocked by the stiffener. The WI result in this scan is significantly clearer than the ZLCC result which is the merit of taking advantage of the amplitude-eliminated phase information after amplitude being eliminated. The T-joint of the stiffener creates a discontinuity at the edge, which is detected by both methods here as shown in the results. The results for LDV location \#2, another scan with wave propagating direction perpendicular to the stiffer, are shown Figure 3.10(b) and Figure 3.11(b). Even though, similarly, most of the wave energy is attenuated by the stiffener, the wave has interacted with the damage before it hit the edge of the stiffener, providing relatively sharper damage imaging than the previous one and the edge of the joint area can be roughly seen in the results.

The ZLCC and WI results for the other two scans with LDV location \#3 are shown in Figure 3.10(c) and Figure 3.11(c), and \#4 in Figure 3.10(d) and Figure 3.11(d) respectively. Since the wave propagating direction is parallel to the stiffener, the portion of wave energy interacting with the damage is significantly higher than the one with propagating direction perpendicular to the stiffener. The interaction between the guided wave and the major delamination is dominant, and 
most of the wave energy is trapped within the major delamination region creating the local resonant vibration. The damage image highlights major delamination in the ZLCC resulting from the standing wave energy, and the WI results show damage region consistent with all other images; however, the major delamination with least stiffness still creates the largest discontinuity in material properties and leads to the concentrated intensity in the results.

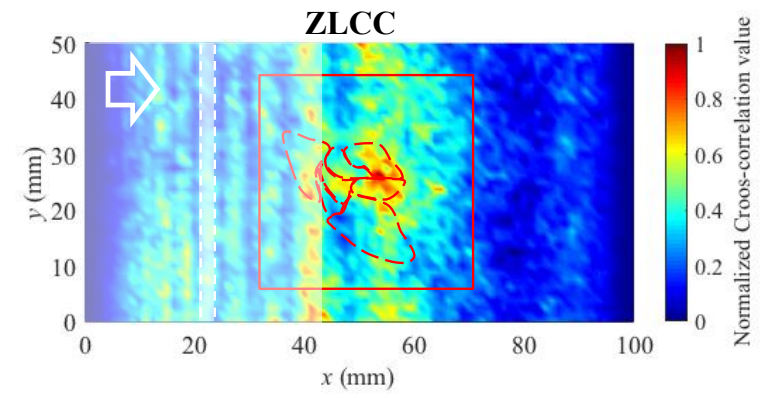

(a)

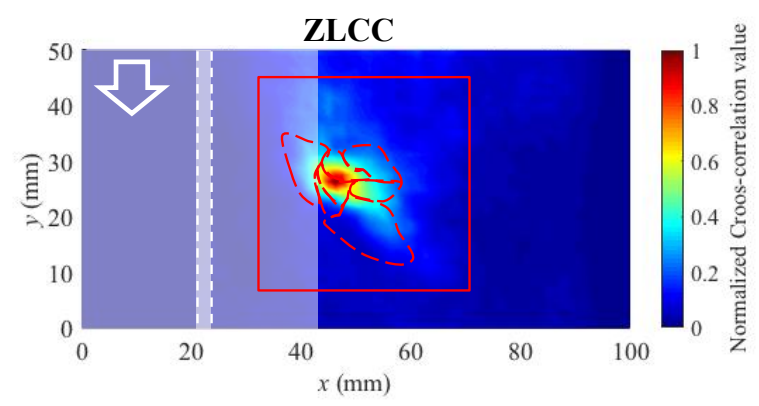

(c)

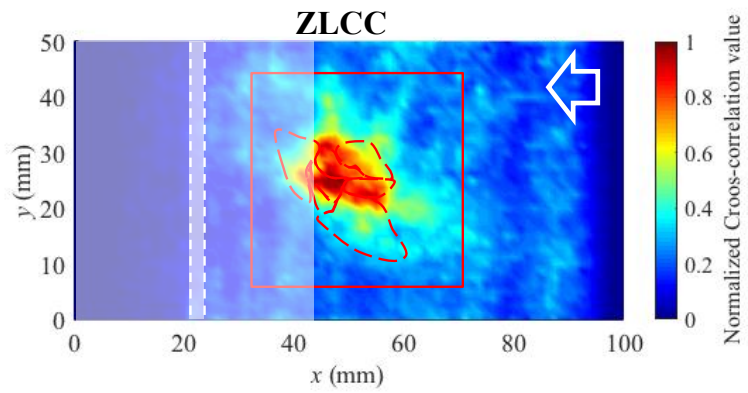

(b)

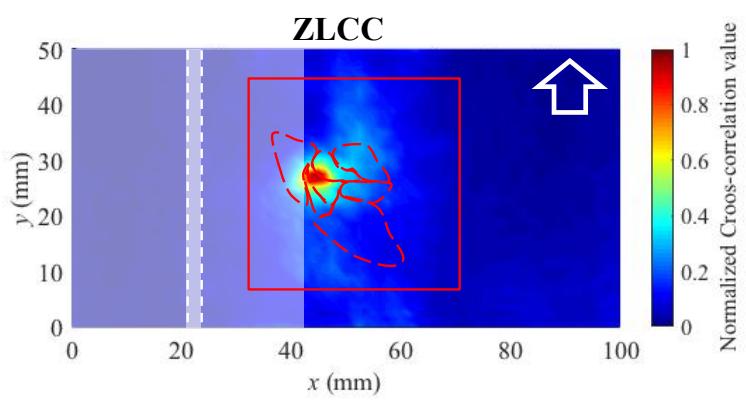

(d)

Figure 3.10. ZLCC results for LDV location and wave propagting direction:

(a) \#1, rightward, (b) \#2, leftward, (c) \#3, downward and (d) \#4, upward. 


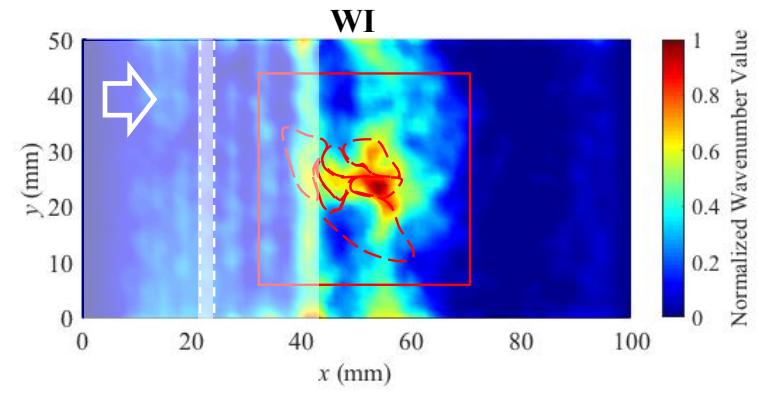

(a)

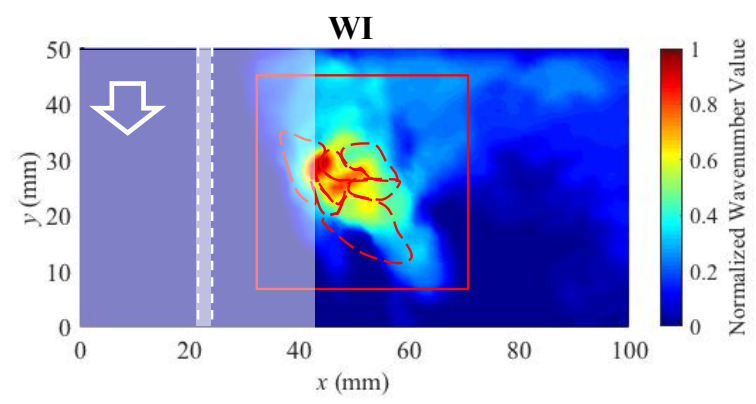

(c)

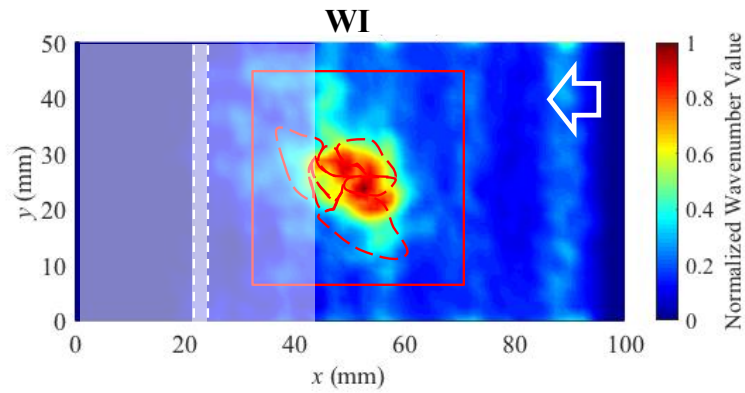

(b)

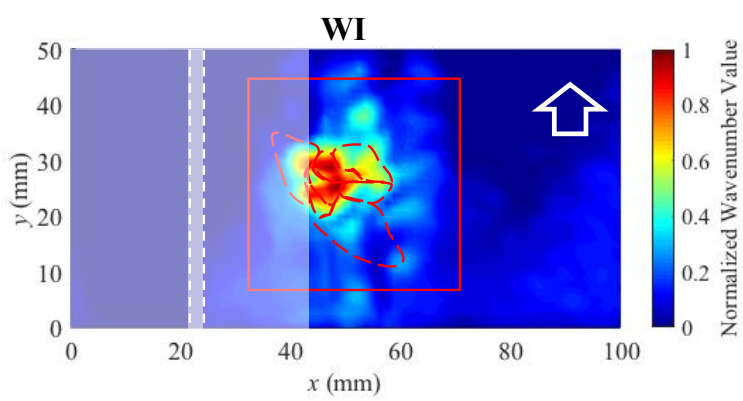

(d)

Figure 3.11. WI results for LDV location and wave propagting direction:

(a) \#1, rightward, (b) \#2, leftward, (c) \#3, downward and (d) \#4, upward.

Generally speaking, both damage imaging methods can effectively image the locations and the shape of the BVID; however, ZLCC yields sharper and more concentrated image than WI methods when the guided wave is less influenced by the stiffener, since ZLCC method is based on not only the disturbance and reflection at the edge of the damage but also the standing wave in the damage region, which is less sensitive to the minor delamination without significant local resonant behavior and highly dependent to the wave propagation direction. WI is mainly based on the changes of material properties instead of the interaction at the damage location, providing better detection capability for minor delamination and small defects when the guided wave is attenuated and disturbed by the stiffener. However WI is also very sensitive to the noise, especially in the 
derivation of phase information. In contrast, ZLCC provides clear and sharp damage images with less noise, and the WI results are more consistent to the C-scan image, however, with less quality and sharpness. Even though the detection resolution for both methods is the same, since two algorithms are based on the same reconstructed wavefield, WI has better sensitivity than ZLCC when the attenuation of the wave amplitude is not negligible. Because WI is only operated in time domain instead of in frequency domain as ZLCC is, it shows better computational efficiency for the large amount of samples.

For simple damage types and structures, the damage detection using instantaneous wavenumber method to show the damage can be quantitative [4.21]. But in this paper the sandwich composite with real impact damage is very complex to derive theoretical wavenumber value. The method can be improved to achieve quantitative in the future study with the assistance of mode separating and wavenumber filtering techniques. The WI technique provides damage imaging results that is consistent with the $\mathrm{C}$-scan images, yielding accurate damage localization with higher sensitivity to the property changes. But it sacrifices the signal-to-noise ratio and the quality of image for the improvement of damage detection. The limitation for $\mathrm{WI}$ is the same as all other imaging condition using wavefield analysis, that the damages need to be located within the scan region, and it is better to be not around the boundaries of the region.

\subsection{Summary and Conclusion}

A guided wave-based damage imaging condition is developed and proposed in this paper. The main aim of this paper is to identify and visualize the impact damage that occurs in the vicinity of a stiffener, which significantly increases the complexity for analyzing the wavefield, by employing the proposed damage imaging condition. The stiffened curved composite sandwich panel used in 
this paper was a section of aileron on a Boeing C-17 Globemaster III military transport aircraft that has been in service for over thirty years. The panel was impacted at NASA Langley Research Center and the impact damage was characterized by an ultrasonic C-scan system for preliminary study and references. The guided wave was excited by a Nd:YAG Q-switched pulse laser and captured by a laser Doppler vibrometer (LDV), and the wavefield images were reconstructed from the three-dimensional data matrix $w(x, y, t)$. Four laser scans were performed with four LDV locations to generate wavefield from these orthogonal directions.

Riesz transform (RT) is a genuine and isotropic generalization of two-dimensional Hilbert transform. For the space-wavenumber, RT is considered as a viable image processing technique and is introduced to the application in non-destructive inspection (NDI) in this paper. By introducing RT to the guided wave analysis, the un-uniformity and inconsistency of wave intensity can be eliminated, and only the phase information is considered can be of value to increase the sensitivity. The material/geometry discontinuity can be shown by observing the change in wave vector. The proposed new imaging condition, named wavenumber index (WI), integrates all the wave vector values within the sampling time of interest and the wave energy threshold filter to highlight all the material/geometry discontinuities. Because of the high sensitivity in phase information, WI improves the accuracy of damage localization but sacrifice the image quality and signal-to-noise ratio after taking derivative to get the instantaneous wave vector. It also shows consistent damage images for all sensing locations and the directions of wave propagation in comparison with zero-lag cross-correlation (ZLCC), which is mainly based on the refraction and reflection at the edge of damage area, especially in the complex stiffened curved panel. WI requires no complex wave filtering and mode separation technique, and it is fully employed in time domain, which is computationally efficient and robust. 
To conclude, the WI via RT developed in this paper provides better accuracy and consistency in damage imaging than the ZLCC based on the wave intensity and reflections, which also requires wavenumber filtering operating in freqency-wavenumber domain. However, the high sensitivity of phase information somehow becomes a disadvantage after taking derivative for noisy signals. Denoising can be helpful to improve the image quality, and the image decomposition may be discussed in the future research. The results have shown a good potential of Riesz transform used as a guided wave-based analysis tool for the application in the field of NDI. 


\subsection{Appendix: C-scan Result}

The C-scan results with respect to the depth beneath the impact location shown in Figure 3.12 are not evenly distributed throughout the thickness for demonstrating clear results only.

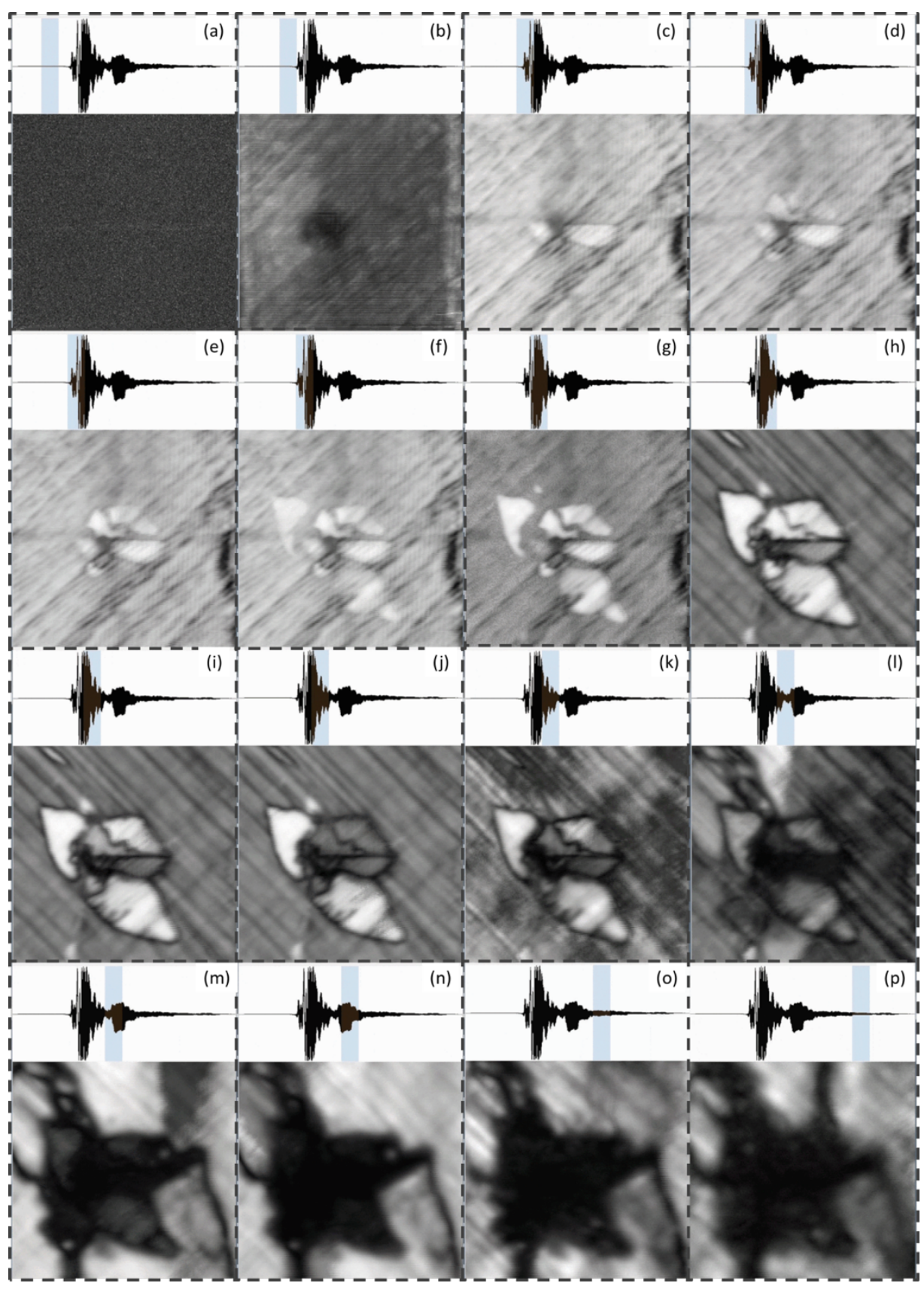

Figure 3.12. The C-scan result shows all the delaminations at different depths beneath the impact location. 


\subsection{References}

[4.1] I.M. Daniel, E.E. Gdoutos, K-A. Wang and J. L. Abot, "Failure modes of composite sandwich beams," International journal of damage mechanics, vol.11, no. 4, pp.309-334, 2002.

[4.2] T.S Lim, S.L. Chang and G.L. Dai, "Failure modes of foam core sandwich beams under static and impact loads," Journal of composite materials, vol.38, no.18, pp.1639-1662, 2004.

[4.3] S. Sánchez-Sáez, E. Barbero, R. Zaera and C. Navarro, "Compression after impact of thin composite laminates," Composites Science and Technology, vol.65, no.13, pp.1911-1919, 2005.

[4.4] F.G. Yuan, ed, Structural Health Monitoring (SHM) in Aerospace Structures, Woodhead Publishing, 2016.

[4.5] C.Y. Chang and F.G. Yuan, "Extraction of guided wave dispersion curve in isotropic and anisotropic materials by Matrix Pencil method," Ultrasonics, vol.89, pp.143-154, 2018.

[4.6] J.B. Ihn and F.K. Chang, "Pitch-catch active sensing methods in structural health monitoring for aircraft structures," Structural Health Monitoring, vol.7, no.1, pp.5-19, 2008.

[4.7] Jiaze He and F.G Yuan, "Lamb-wave-based two-dimensional areal scan damage imaging using reverse-time migration with a normalized zero-lag cross-correlation imaging condition," Structural Health Monitoring, vol.16, no.4, pp.444-457, 2017.

[4.8] S.B. Kim, C.G. Lee, J.W. Hong, H.W. Park and H. Sohn, ”Applications of an instantaneous damage detection technique to plates with additional complexities," Journal of Nondestructive Evaluation, vol.29, no.3, pp.189-205, 2010. 
[4.9] X. Yu, Z. Fan, M. Castaings and C. Biateau, " Feature guided wave inspection of bond line defects between a stiffener and a composite plate," NDT \& E International, vol.89, pp.44$55,2017$.

[4.10] M. Ruzzene, S. M. Jeong, T. E. Michaels, J. E. Michaels, and B. Mi, "Simulation and Measurement of Ultrasonic Waves in Elastic Plates using Laser Vibrometry," AIP Conference Proceedings, Vol.760, no.1, pp.172-179, 2005.

[4.11] A. Zak, M. Radzienski, M. Krawczuk, and W. Ostachowicz, "Damage Detection Strategies based on Propagation of Guided Elastic Waves," Smart Materials and Structures, vol.21, p.035024, 2012.

[4.12] H. Sohn, D. Dutta, J.Y. Yang, H.J. Park, M. DeSimio, S. Olson, and E. Swenson, "Delamination detection in composites through guided wave field image processing," Composites science and technology, vol.71, no.9, pp.1250-1256, 2011.

[4.13] Y.K. An, B. Park, and H. Sohn, "Complete noncontact laser ultrasonic imaging for automated crack visualization in a plate," Smart Materials and Structures, vol.22, no.2, p.025022, 2013.

[4.14] B. Park, Y.K An, and H. Sohn, "Visualization of hidden delamination and debonding in composites through noncontact laser ultrasonic scanning," Composites science and technology, vol.100, pp.10-18, 2014.

[4.15] M. Ruzzene, "Frequency-wavenumber domain filtering for improved damage visualization," In Ultrasonic And Advanced Methods For Nondestructive Testing And Material Characterization, pp.591-611, 2007.

[4.16] M.S. Harb and F.G. Yuan, "Impact damage imaging using non-contact ACT/LDV system," Structural Health Monitoring, vol.15, no.2, pp.2567-2574, 2015. 
[4.17] M.S. Harb and F.G. Yuan, "Damage imaging using non-contact air-coupled transducer/laser Doppler vibrometer system," Structural Health Monitoring, vol.15, no.2, pp.193-203, 2016.

[4.18] D. Girolamo, H.Y Chang, and F.G. Yuan, "Impact Damage Visualization in a Honeycomb Composite Panel through Laser Inspection using Zero-lag Cross-correlation Imaging Condition," Ultrasonics, vol.81, pp.152-165, 2018.

[4.19] M.D. Rogge and C.A. Leckey, "Characterization of impact damage in composite laminates using guided wavefield imaging and local wavenumber domain analysis," Ultrasonics, vol.53, no.7, pp.1217-1226, 2013.

[4.20] P.D. Juarez and C.A. Leckey, "Multi-frequency local wavenumber analysis and ply correlation of delamination damage," Ultrasonics, vol.62, pp.56-65, 2015

[4.21] O. Mesnil, C.A. Leckey and M. Ruzzene, "Instantaneous and local wavenumber estimations for damage quantification in composites," Structural Health Monitoring, vol.14, no.3, pp.193-204, 2015.

[4.22] E.B. Flynn, S.Y. Chong, G.J Jarmer and J.R. Lee, "Structural imaging through local wavenumber estimation of guided waves," NDT \& E International, vol.59, pp.1-10, 2013.

[4.23] S. Sánchez-Sáez, E. Barbero, R. Zaera, and C. Navarro, ”Compression after impact of thin composite laminates," Composites Science and Technology, vol.65, no.13, pp.1911-1919, 2005.

[4.24] J. He, and F.G. Yuan, "Lamb wave-based BVID imaging for a curved composite sandwich panel,” In AIP Conference Proceedings, vol.1806, no.1, pp.050012, AIP Publishing, 2017.

[4.25] H.Y. Chang and F.G. Yuan, "Impact damage imaging in a curved composite panel with wavenumber index via Riesz transform," Proc. SPIE 10599, Nondestructive 
Characterization and Monitoring of Advanced Materials, Aerospace, Civil Infrastructure, and Transportation XII, p.105990L, 2018.

[4.26] J. Achenbach, Reciprocity in Elastodynamics, Cambridge University Press, 2003.

[4.27] K. Langley, and S.J. Anderson, “The Riesz transform and simultaneous representations of phase, energy and orientation in spatial vision," Vision research, vol.50, no.17, pp.1748$1765,2010$.

[4.28] M. Felsberg and G. Sommer," The monogenic signal," IEEE Transactions on Signal Processing, vol.49, no.12, pp.3136-3144, 2001.

[4.29] N. Wadhwa, M. Rubinstein, F. Durand and W.T. Freeman, "Riesz pyramids for fast phasebased video magnification," In Computational Photography (ICCP), 2014 IEEE International Conference on , pp.1-10, 2014. 


\section{CHAPTER 5 Damage Visualization of Scattered Ultrasonic Wavefield via Integrated High-speed Camera System}

\subsection{Introduction}

Guided wave-based techniques have shown promise in nondestructive inspection (NDI) and structural health monitoring (SHM) to reduce the risks of catastrophic failures of aircrafts [5.1]. Since the guided wave signal from a damaged structure contains the temporal and spatial information of the defect source, proper algorithms can be implemented for damage detection and visualization [5.2]. The techniques can be broadly divided into active sensing and passive sensing, and guided wave information can be obtained for both methods through either contact, e.g., discrete guided wave signal from a piezoelectric (PZT) sensor array [5.3], or non-contact methods, e.g. wavefield scanning using Nd-YAG pulse laser [5.4], and laser Doppler vibrometer (LDV) [5.5]. In passive sensing, guided wave can be indirectly obtained by taking advantage of the ambient disturbance generated naturally from turbulent flows during the flight [5.6], or directly obtained by impact source or by acoustic emission from the failure of the component [5.7], in which potential defect location is buried in the signal. In active sensing, the guided wave is excited/measured directly using actuator/sensor, and discrete data for reconstructing wavefield propagation is acquired through regional scanning on the structure for accurately locating and visualizing the damage [5.2]. Even though the performance and limitation of these techniques varies, one common drawback is that the systems require significant set-up time. Further collecting signal or scanning wavefield can be very time-consuming.

Optical measurement via high-speed cameras is one of the most viable means that can circumvent these problems for both active and passive sensing, as the IC technology rapidly advances following Moore's law, the new high-speed cameras nowadays are promising good potentials to 
be able to capture propagating guided wave on the surface of the objects. The wavefield reconstructed from camera images can provide the most spatially continuous sampling result compared to any other measurement methods with discrete sensing locations. The optical measurement combined with algorithms for both passive and active damage visualization allows real-time health monitoring and rapid damage detection of the aircraft, and thus reducing frequent scheduled maintenance and downtime. Even though currently the measurement using camera and LDV system cost roughly the same amount of time and the advantage of using camera is not significant, the computing time for optical measurement basically is only limited by the processing speed, and it will improve as the development in technology [5.8]. In this study, for the first time, an optical guided acoustic wave-based damage visualization technique using high-speed camera was established.

The high-speed camera enables capturing images of rapid wave propagation on a surface of the structure excited by a PZT actuator, and two in-plane displacement components was further extracted using digital image correlation (DIC). Due to the current limitation in the performance of high-speed cameras, strategies were needed because of the difficulty on the measurement of the acoustic wave signal with extremely high frequency and the small magnitude of wave deformation. In terms of solving the current hardware problem, the sample interleaving (to increase equivalent sampling frequency) and image stitching (to increase equivalent field-of-view (FOV)) were both employed in wavefield reconstruction. Both techniques are straightforward, intuitive and have been applied to several experiments with long history for increasing equivalent temporal and spatial sampling frequency. For the application in optical measurement of guided wave, Schaeffer et al. [5.9] have used both techniques to measure the in-plane wave in a metamaterial [5.9], and Darnton and Ruzzene [9] have employed the sample interleaving in a qualitative study on a 
fiberglass plate using a high-speed camera [5.10], but the damage visualization and quantitative study had not been conducted yet.

The integrated scanning system was developed to improve the signal quality on the affordable hardware. Since the speckle quality applied on the specimen is critical for displacement extraction [5.11] and the speckle and FOV size are highly related [5.12], the optimization of the speckle is needed and the surface treatment and perfect primer are also inevitable to increase the signal quality [5.13]. The measured images are then analyzed to extract the in-plane displacement information using VIC-2D software by Correlated Solutions, Inc.. The damage imaging condition technique employed to visualize the damage is named wavenumber index (WI), which was developed and had been successfully applied to composites by Chang and Yuan [5.14]. The WI technique showed the damage image by analyzing the local material/geometrical discontinuity from the guided wavefield reconstructed from the LDV data. In this study, the technique was improved to eliminate the misalignment from stitched image data taken from the high-speed camera. Combined with both active and passive damage visualization technique, this work done on the integration and optimization of high-speed camera scanning system and the new damage imaging condition shows a new revolution to achieve the real-time NDI and SHM.

In the next section, the experimental setup of the camera system is presented. Following that, the wavefield image reconstructed from the displacement field using DIC is illustrated, and the damage image generated by the improved WI technique is shown and explained. A short conclusion is made at the last section. 


\subsection{Experiment Setup}

The high-speed camera used in the research was a Photron Mini AX-200, and the maximum resolution was $1,024 \times 1,024$ pixels at 6,400 frame per second, which yields the limited data requisition rate at roughly 10 gigabytes per second, so the resolution will be reduced as the frame rate increases. For the balance between image resolution and sampling frequency, the camera was set at $384 \times 384$ pixels at 36,000 frame per second. The camera and two Zaila Daylight LEDs were mounted on the two-dimensional translational stage for precise position control with minimum step in $0.01 \mathrm{~mm}$. Each LED was equipped with a $10^{\circ}$ holographic lens and a low-light-loss diffusion filter for the flat illumination. The images were captured using Photron FASTCAM Viewer, and the function generator (Tektronix AFG3022C) was synchronized with the camera. The specific time-delay was set for each test, and the time-delayed signal was then amplified with a voltage amplifier (Krohn-Hite 7602M) for the excitation using a PZT actuator. The PZT actuator was an APC product \#81 that was made with material \#850, and it was $25.4 \mathrm{~mm}$ in diameter and $2.1 \mathrm{~mm}$ in thickness. The excitation signal was initially chosen as a continuous sine wave with the frequency at $14 \mathrm{kHz}$, which is slightly lower than the theoretical Nyquist frequency $18 \mathrm{kHz}$.

Due to the limited performance, in order to ensure the measurability of the guided wave on the specimen for the camera, low Young's modulus and high density are required for low wave speed and large displacements. Thus a high-density polyethylene (HDPE) plate was selected for the proof of concept. The HDPE plate was $300 \mathrm{~mm} \times 300 \mathrm{~mm}$ and $0.85 \mathrm{~mm}$ in thickness with the density $950 \mathrm{~kg} / \mathrm{m}^{3}$ and Young's modulus $0.8 \mathrm{GPa}$, so that the theoretical dispersion relation could be calculated with the given parameters. The PZT actuator was placed at $85 \mathrm{~mm}$ away from the edge of the scan area, which is a $70 \mathrm{~mm} \times 70 \mathrm{~mm}$ square region located at the center of the plate. A 12 
$m m \times 25 \mathrm{~mm}$ rectangular magnet attached to the back of the plate and located at the center of the scan area to mimic as a damage. The schematic experimental setup is illustrated in Figure 4.1.

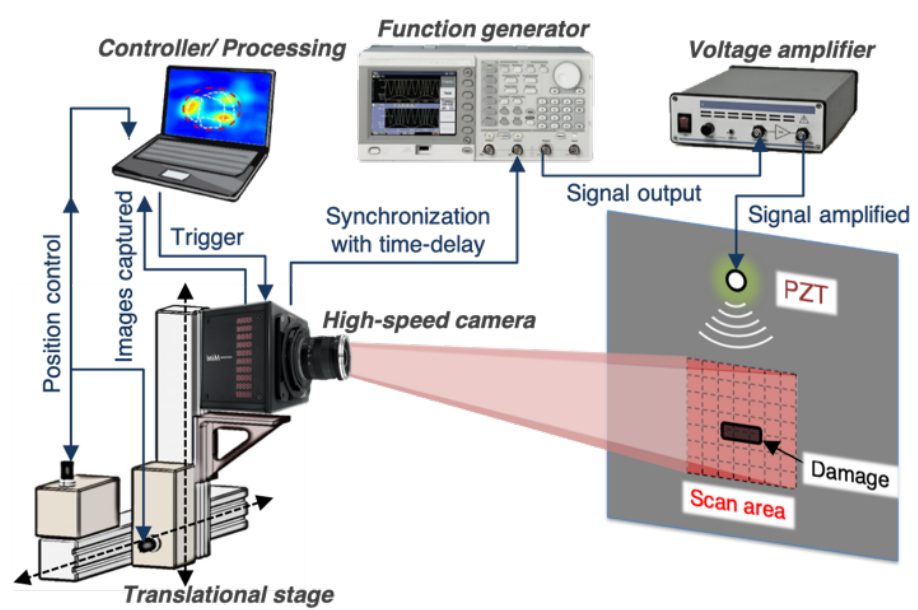

(a)

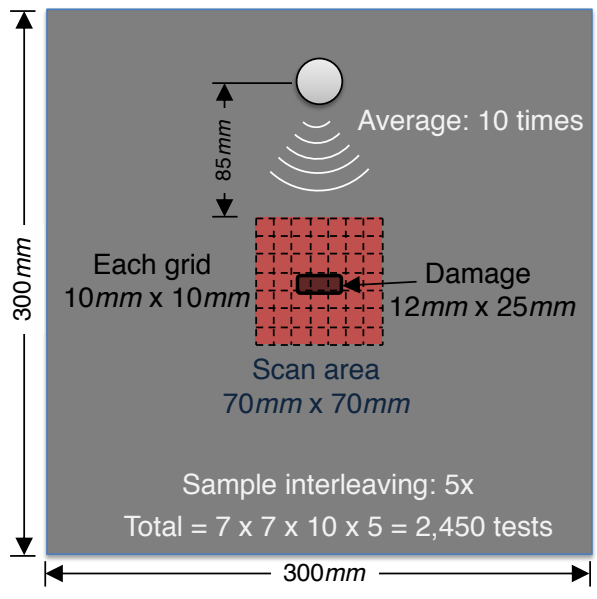

(b)

Figure 4.1. (a) The schematic system layout of experimental setup, and

(b) the illustration of specimen setup in the test

Since the field of view (FOV) for each measurement was limited by the small targeted displacement, the entire scan region was split into $7 \times 7$ sections, each of which was measured separately. The individual measurements were further stitched together to reconstruct the signal as if it were measured within the entire scan area. In addition, to increase the equivalent sampling frequency with the current limited frame rate, a sample interleaving technique was conducted by repeating the signal excitation with a specific time-delay. The time-delayed signals were interleaved sequentially to reconstruct a signal that was measured equivalently with multiple times sampling frequency. In this experiment, the equivalent sampling frequency was increased by 5 times by employing the sample interleaving technique. Also, because of the low signal-to-noise ratio led by the subtle displacement in the experiment, each measurement was repeated ten times 
and then averaged to eliminate incoherent noise. Therefore the total amount of measurement was 2,450 times in order to overcome the limitation of hardware instead of capturing the whole field signal at once. The DIC software used for extracting in-plane displacement field in the experiment was VIC-2D by Correlated Solutions, Inc., and the maximum in-plane resolution can reach up to $0.00001 \times$ FOV. Each measurement was applied with DIC analysis to obtain the displacement data before stitching, interleaving and averaging

Before measuring the wavefield by high-speed camera, the wavefield was also obtained by a LDV (Polytec OFV-505), and the damage image could be visualized by employing a damage imaging condition for references. The scanning region for LDV was the same $70 \mathrm{~mm} \times 70 \mathrm{~mm}$ square with $2 \mathrm{~mm}$ spacing between sampling points, leading to total $36 \times 36$ points in an experiment. The reflective tape was applied above the scanning area for better signal quality, and the base coat and speckles were applied onto the same area after the LDV scanning. After several tests and evaluations, the paint selected for best quality for the base coat was the Rust-Oleum flat-white paint-primer, and the speckles were applied with a Harder \& Steenbeck Infinity CR plus airbrush with a nozzle in $0.15 \mathrm{~mm}$ for the minimal speckle size.

\subsection{Logarithmic Value of Wavenumber Index (WI)}

In this article, the damage was visualized with the wavenumber index (WI) imaging condition via Riesz transform that was previously developed by Chang and Yuan [5.14]. However, unlike delamination or other damage types in composite structures that weaken the structure, the magnet attached to the plate added extra mass and stiffness to the region instead of reducing them. Thus the interaction between the guided wave and the damage is different from the behavior with 
delamination. Moreover, the misalignment between each stitched sub-section caused a significant larger discontinuity (in different order) of wavenumber at the boundaries than the damage region, which led to the damage image with poor quality. In order to remove the dominance of the discontinuity in the wavenumber index caused by image stitching, the damage imaging condition was slightly modified to improve the visualization of the simulated damage (magnet) on the HDPE plate.

The modified WI is shown in the normalized value as expressed in Eq.(5.1), and the nonnormalized WI calculated using logarithmic accumulated wavenumber value is expressed in Eq.(5.2), where $t_{i}$ is the initial time instant of the interested segment of sampling, $T$ is the total period, and $k(x, y, t)$ is the instantaneous wavenumber derived via Riesz transform that was previously employed by Chang and Yuan [5.14]. In this case, the accumulated wavenumber value is in logarithmic scale in order to eliminate the misalignment problem from stitching, since the values at each boundary are significantly higher than the damaged area. The modified WI method was also applied to the wavefield measured by the LDV. Even though the LDV data was not affected by stitching, the damage image was still generated with the modified method for fair comparison. The result using the logarithmic values was clearer and more concentrated to the center of the damage than the original method, so the visualized damage region was slightly smaller than the real damage region.

$$
\begin{gathered}
\overline{\mathrm{WI}}(x, y)=1-\frac{\mathrm{WI}(x, y)}{\operatorname{Max}[\mathrm{WI}(x, y)]} \\
\mathrm{WI}(x, y)=\log \left[\sum_{t=t_{i}}^{T} k(x, y, t)-\operatorname{Min}\left(\sum_{t=t_{i}}^{T} k(x, y, t)\right)\right]
\end{gathered}
$$




\subsection{Results and Comparison- Signal Frequency below the Nyquist Frequency}

Due to the extremely low magnitude of the displacements excited from the PZT actuator, in order to visualize the reconstructed acoustic wavefield, the displacement extracted by DIC required a bandpass frequency filter for enhancing the signal quality. For DIC measurement, there were two wavefield reconstructed with $x$-direction and $y$-direction displacement, and the frequency spectrums of pre-filtered signal for $y$-directions were plotted at an arbitrary location via fastFourier transform (FFT) in Figure 2(a). Even though the signal frequency at $14 \mathrm{kHz}$ was shown clearly in the frequency spectrum, which indicated the presence of the wavefield in the extracted displacement data, the wavefield was not able to be visualized in time domain because of the ambient noise and unwanted misalignment caused by the sample interleaving. Significant peaks in the frequency spectrum induced by the misalignment of interleaved data could be shown at $0 \mathrm{~Hz}$, $36 \mathrm{kHz}$ and $72 \mathrm{kHz}$. After applying the filter, the signal frequency was perfectly shown in Figure 2(b) so that the reconstructed wavefield could be visualized in time domain, and the interaction between the guided wave and the damage could also be observed. 


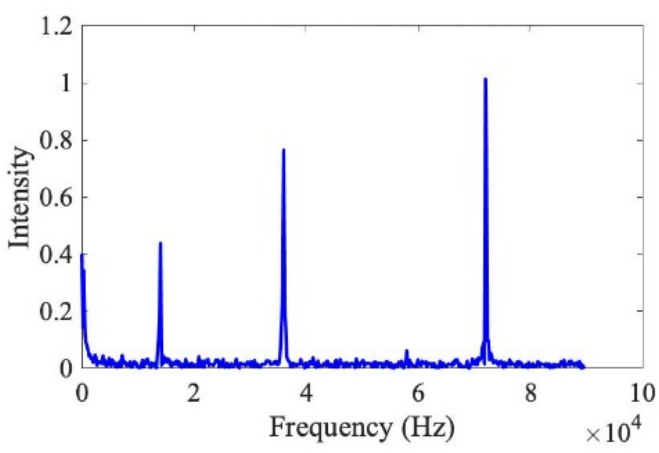

(a)

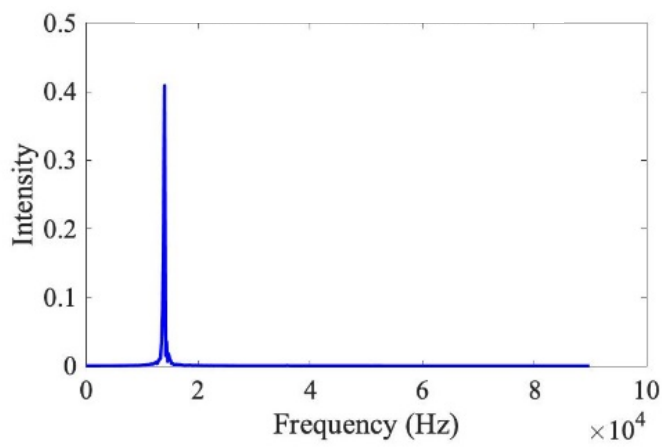

(c)

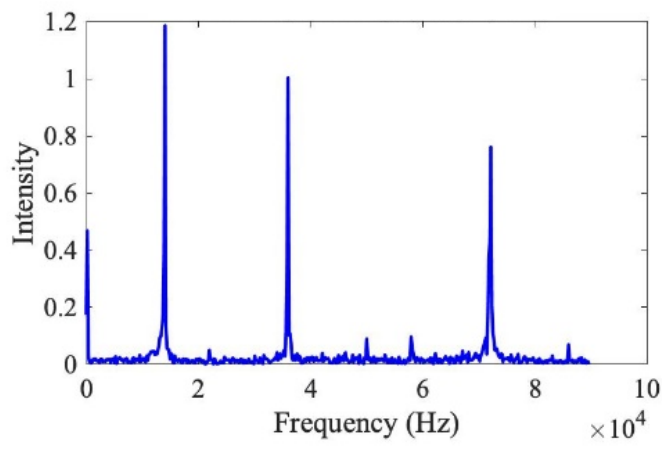

(b)

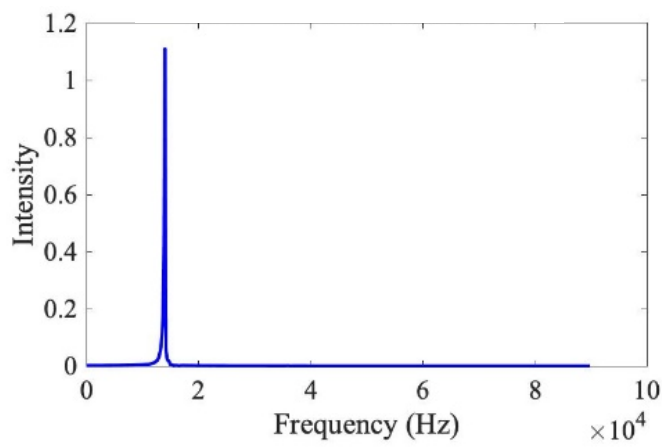

(d)

Figure 4.2. Frequency spectrum for $14 \mathrm{kHz}$ excitation at an arbitrary point before filtering for (a) $x$-displacement, and (b) y-displacement; after filtering for (c) $x$-displacement, and (d) y-displacement

Since a single LDV could only measure the out-of-plane motion and a single camera could only measure in-plane motion, the detectable wavefield obtained from the two methods belong to different wave modes. The theoretical wave speed and wavelength for each modes were calculated from the material properties of the HDPE plate. For $A_{0}$ mode, the group velocity $c_{\mathrm{g}}=338 \mathrm{~m} / \mathrm{s}$ and the corresponding wavelength $\lambda=13 \mathrm{~mm}$; for $S_{0}$ mode, the group velocity $c_{\mathrm{g}}=1,423 \mathrm{~m} / \mathrm{s}$ and the corresponding wavelength $\lambda=100 \mathrm{~mm}$; for $S H$ mode, the group velocity $c_{\mathrm{g}}=739 \mathrm{~m} / \mathrm{s}$ and the corresponding wavelength $\lambda=53 \mathrm{~mm}$. 


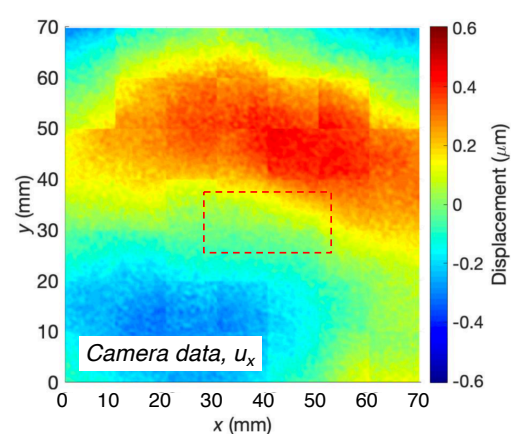

(a)

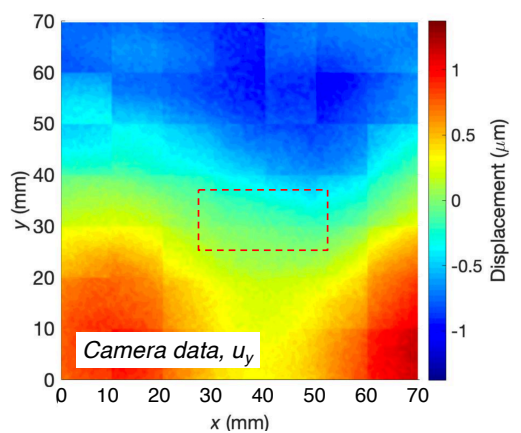

(b)

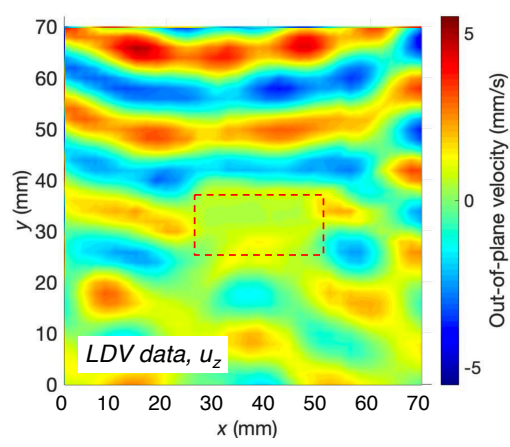

(c)

Figure 4.3. The wavefield snapshot at $800 \mu \mathrm{s}$ for (a) $u_{x}$, (b) $u_{y}$ from camera, and (c) $u_{z}$ from LDV. The red dotted rectangle indicates the simulated damage at the back of the plate.

For DIC measurement, there were two wavefields reconstructed with displacements in both $x$ - and $y$-directions. By comparing the wavelength and wave speed of the measured wavefield to the theoretical values, it could be concluded that the reconstructed wavefield using $y$-displacement obtained from DIC was in $S_{0}$ mode, and the one using $x$-displacement obtained from DIC was in $S H$ mode. Similarly, the wavefield measured by the LDV could be readily identified as $A_{0}$ mode by comparing with the theoretical values, and the wave propagation and interaction with damage could also be clearly observed. The snapshots of each wavefield at $800 \mu$ s are shown in Figure 3. Since the scan area was $70 \mathrm{~mm} \times 70 \mathrm{~mm}$, which was of comparable size as the wavelength of $S_{0}$ mode, as shown in Figure 3(b), the wave propagation was relatively less clear than that in both $\mathrm{SH}$ and $A_{0}$ modes, shown in Figure 3(a) and (c), and the damage region was marked with red dotted line.

The damage imaging results for the $S H$ mode (from $x$-displacement via DIC), $S_{0}$ mode (from $y$ displacement via DIC) and $A_{0}$ mode (from out-of-plane velocity via LDV) are shown in Figure 4 (a), (b) and (c) respectively, and the damage region is marked with red dotted line. The result 
generated from $\mathrm{SH}$ mode successfully visualize the image that is consistent with the exact location and size of the damage. The result from $S_{0}$ mode shows the image at the correct $y$-coordinate, but the $x$-coordinate is not exactly at the damage region and some image artifacts are shown outside the region. In addition, the damage image from the LDV measurement is clear, consistent with but slightly smaller than the real damage region, probably because of the double-sided tape used for attaching the mass was too thick, soft and slightly smaller.

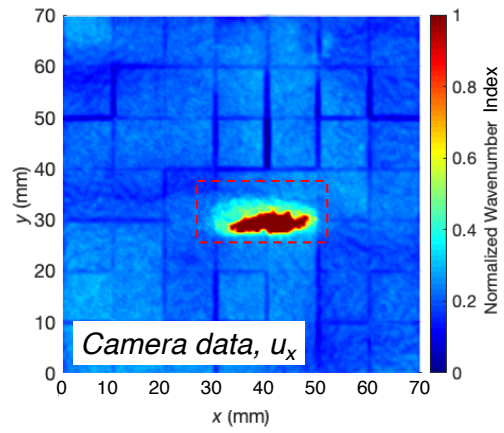

(a)

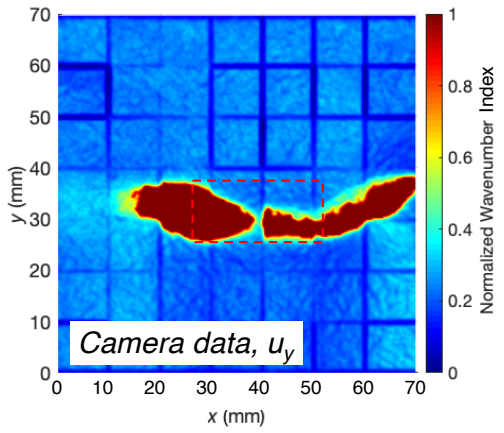

(b)

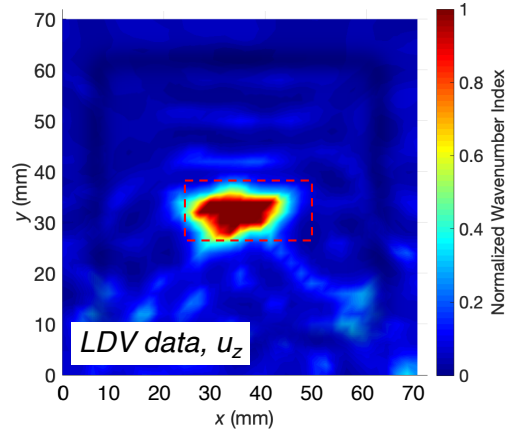

(c)

Figure 4.4. The damage image generated from wavefield in (a) $\mathrm{SH}$ mode from camera, (b) $S_{0}$ mode from camera, and (c) $A_{0}$ mode from LDV. The red dotted rectangle indicates the simulated damage at the back of the plate.

The reason that causes the difference and the error of the damage image generated from $S_{0}$ mode can be possibly categorized into two main reasons: (1) The wavelength and the dimension of the damage (added mass) and (2) the interaction between the damage and wave motion in different modes. First, the wavelength for $S_{0}$ mode is more than eight times of the damage size $12 \mathrm{~mm}$ in $y$ direction which is relatively too large for damage visualization, compared to the wavelength of $S H$ mode. Second, the in-plane motion of $S_{0}$ mode is less sensitive to simulated damage of the mass added to the plate than the out-of-plane motion of $A_{0}$ mode due to the nature of the particle motion 
for different wave modes. Third, since the damage is $25 \mathrm{~mm}$ in $x$-direction and $12 \mathrm{~mm}$ in $y$ direction, the damage has more interaction with the $S H$ mode moving in $x$-direction than the $S_{0}$ mode. Further research for the interaction between $S H$ and $S_{0}$ wave and the real delamination in composites will be conducted. From the reasons mentioned above, it is reasonable and understandable that the image from $S_{0}$ mode has less accurate result.

\subsection{Results and Comparison- Signal Frequency above the Nyquist Frequency}

For the horizontal damage, it can be successfully visualized with the SH mode of the reconstructed wavefield, since the wavelength of SH mode is roughly two times of the horizontal dimension of the rectangular magnet, and the damage is barely detectable for this wavelength value. However, for the same magnet piece with other orientation, the projected length of the damage in horizontal direction will be reduced, and the wavelength in SH mode will become too large for the damage visualization, which leads to a unsuccessful broken damage image. Therefore, for the visualization of smaller damages or damages with other shape and orientations, the signal frequency needs to be increased for reducing the wavelength.

The signal frequency was then further increased to $28 \mathrm{kHz}$, which is above the Nyquist frequency of the sampling frequency of the high-speed camera at $36 \mathrm{kHz}$. Since the wave speed of $S H$ mode is independent to the signal frequency, and the $S_{0}$ mode basically remain the same for low frequency range, the adjusted signal frequency was chosen as $28 \mathrm{kHz}$ based on this nature of the dispersion relations of guided wave, so that the wavelength is simply reduced into half by doubling the signal frequency. The frequency spectrums of the wave signal at an arbitrary point within the scanning region before/after bandpass filtering are shown in Figure 4.5. Similarly, significant 
peaks in the frequency spectrum induced by the misalignment of interleaved data before bandpass filtering were shown at $0 \mathrm{~Hz}, 36 \mathrm{kHz}$ and $72 \mathrm{kHz}$ in Figure 4.5 (a) and (b). The signal frequency above the Nyquist frequency for the camera was successfully captured and identified with the equivalent five times sampling frequency that was enhanced by the sampling interleaving technique. With the bandpass filter, the misalignment can be perfectly removed and the signal frequency can be extracted for the wavefield reconstruction.

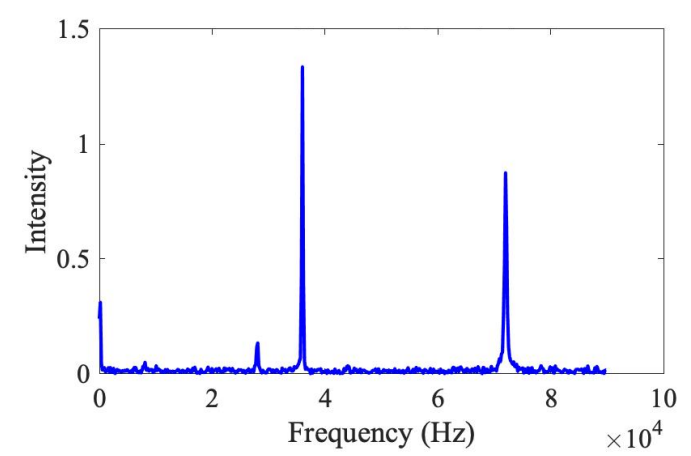

(a)

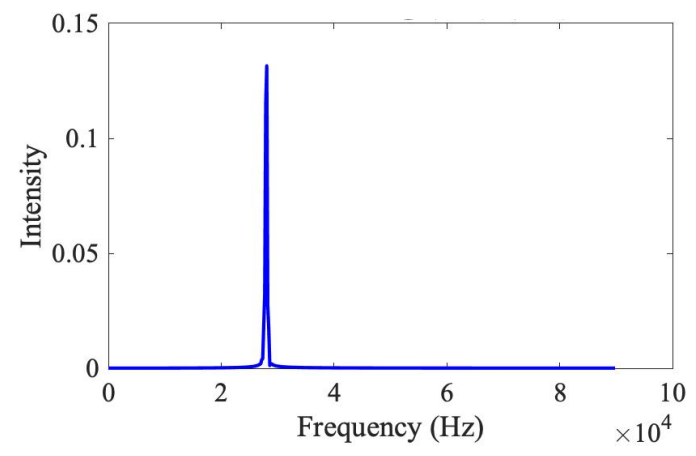

(c)

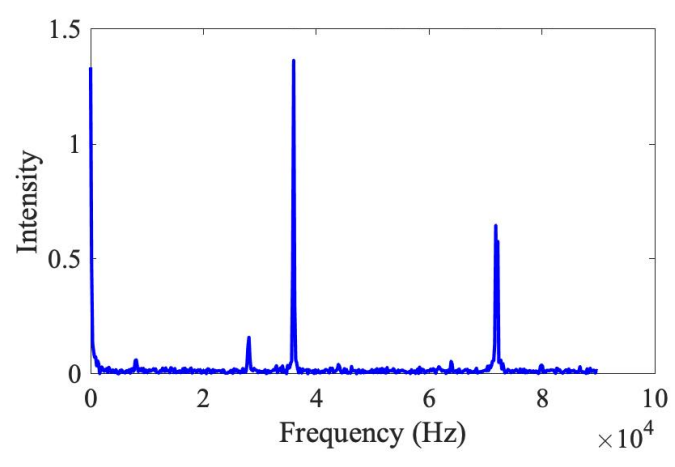

(b)

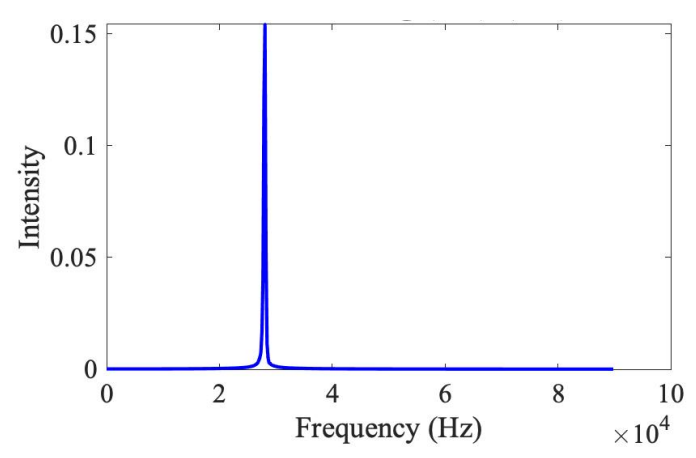

(d)

Figure 4.5. Frequency spectrum for $28 \mathrm{kHz}$ excitation at an arbitrary point before filtering for (a) $x$-displacement, and (b) y-displacement; after filtering for (c) $x$-displacement, and (d) y-displacement 
When the signal frequency was increased to $28 \mathrm{kHz}$, for $S_{0}$ mode, the group velocity remained at $c_{\mathrm{g}}=1,423 \mathrm{~m} / \mathrm{s}$ so the corresponding wavelength became $\lambda=51 \mathrm{~mm}$; for the fundamental $\mathrm{SH}$ mode, the group velocity is independent to the frequency so it remained at $c_{\mathrm{g}}=739 \mathrm{~m} / \mathrm{s}$ and the corresponding wavelength became $\lambda=26 \mathrm{~mm}$. The wavelengths for both wave modes were both reduced into half, but the projected length in horizontal direction also became half with a 60 degree tilt, and the projected length in vertical direction changed from $12 \mathrm{~mm}$ to $21.7 \mathrm{~mm}$, which is slightly less than a half of the wavelength of $S_{0}$ mode. The damage visualization results are shown in Figure 4.6. Figure 4.6 (a) shows the damage image created using the $S H$ mode reconstructed from displacement in $x$-direction, and Figure 4.6 (b) shows the result created using the $S_{0}$ mode reconstructed from displacement in $y$-direction. The damage image generated using the wavefield in SH mode perfectly indicates the location and geometry of the tilted magnet as the imitated damage; however, the result from S0 mode failed to visualize the hidden damage and only shows the location of the top boundary of the damage region.

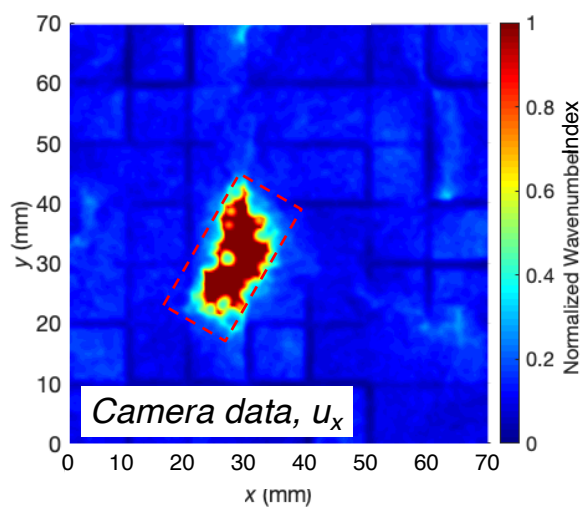

(a)

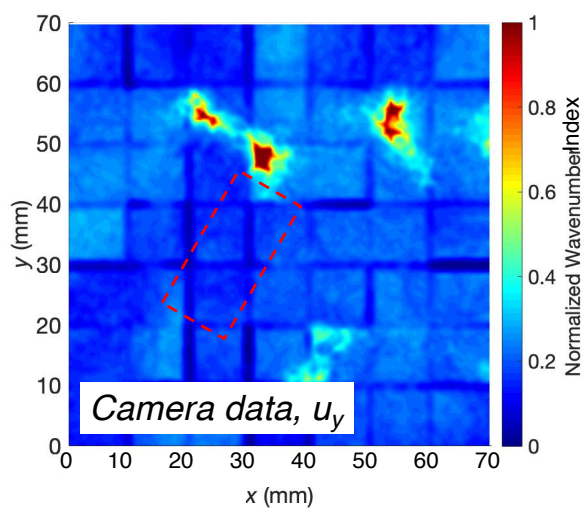

(b)

Figure 4.6. The damage image generated from reconstructed wavefield via camera in (a) $\mathrm{SH}$ mode, (b) $S_{0}$ mode. The red dotted line indicates the simulated damage at the back of the plate. 
The rectangular magnet was then replaced by a circular aluminum disk with $25.4 \mathrm{~mm}$ in diameter and $10 \mathrm{~mm}$ in thickness which is more than sufficient to create the discontinuity for mimicking the damage. The circular disk provided the equal dimension in both $x$ - and $y$-directions for studying the sensitivity of both wave modes for damage visualization, which is shown in the Figure 4.7. Similarly, Figure 4.7 (a) shows the damage image created using the $S H$ mode reconstructed from the displacement in $x$-direction, and Figure 4.7 (b) shows the result created using the $S_{0}$ mode reconstructed from the displacement in $y$-direction. The damage image from the $S H$ mode still perfectly shows the imitated damage region and location, but the result from $S_{0}$ mode is still broken and only indicates the top of the damage boundary.

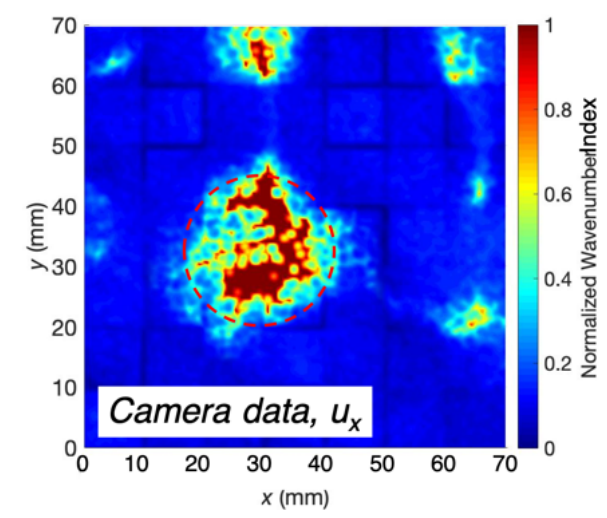

(a)

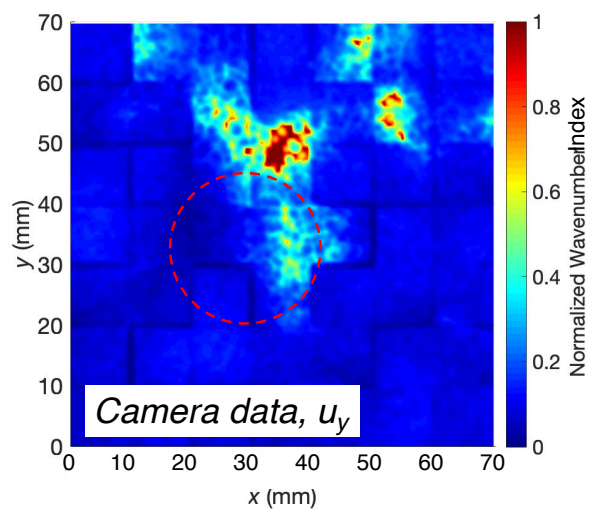

(b)

Figure 4.7. The damage image generated from reconstructed wavefield via camera in (a) $\mathrm{SH}$ mode, (b) $S_{0}$ mode. The red dotted line indicates the simulated damage at the back of the plate.

By increasing the signal frequency from $14 \mathrm{kHz}$ to $28 \mathrm{kHz}$, the wavelengths were reduced to half for both $S H$ mode and $S_{0}$ mode, and the resolution and sensitivity for damage visualization were supposed to be increased as the reduction in wavelength. However it still failed to visualize either the rectangular damage or the circular damage using the $S_{0}$ mode, while it maintained the same accuracy and good agreement with the damage size and location using $S H$ mode. It makes more 
sense to conclude that the $S_{0}$ mode is not sensitive to detect and visualize the added mass on the plate, at least for this experimental condition with very low frequency range. Even though only the results generated from $\mathrm{SH}$ mode successfully visualized the damage on the HDPE plate, it was still the first time that the damage was visualized using the wavefield reconstructed using a high-speed camera to measure the in-plane displacement.

\subsection{Summary and Conclusion}

In this article, a major breakthrough on the application of high-speed camera with digital image correlation (DIC) in nondestructive inspection (NDI) and structural health monitoring (SHM) was achieved for capturing guided acoustic wavefield with the aim of damage imaging. The damages

were successfully visualized using the scattered guided wavefield measured via a high-speed camera with DIC system. By conducting the sampling interleaving and image stitching technique, the integrated system was able to achieve about 250 times of original data requisition rate specified by the camera. Moreover, the experimental parameters and speckle pattern were specifically designed and studied for the best signal quality for measuring sub-micron scale displacements, so that the wavefield sensing and reconstruction became possible. The concept using camera to measure the in-plane wave modes and visualize the hidden damage was demonstrated on a high density polyethylene (HDPE) plate, which is a soft polymer plate with less than one percent of stiffness of regular CFRP composite structures, in order to introduce large deformation and low wave speed, so that the wavefield of both $S H$ and $S_{0}$ mode were able to be successfully detected using an affordable high-speed camera. 
The phase-based damage imaging condition, wavenumber index (WI), that was previously developed and applied to the visualization of delamination in composites through a laser scanning system, was employed to demonstrate the concept of the damage visualization on the reconstructed wavefield using a high-speed camera. Due to the significant local discontinuity in wavenumber induced by the spatial misalignment from image stitching, the WI technique was further enhanced with the introduction of logarithmic value to eliminate the misalignment and other ambient noise for improving the accuracy and imaging quality. Even though the signal quality was sacrificed for the signal frequency above the Nyquist frequency, the WI technique was successful in the damage visualization thanking to its high sensitivity to the material/geometrical discontinuities. Complex frequency/wavenumber filters were not required in the damage imaging processing, making the WI technique a robust and computationally efficient solution for the guided wave-based damage visualization.

The algorithms and techniques conducted in this article successfully demonstrated the visualization of hidden damage in a plate structure using WI damage imaging condition via highspeed camera with digital image correlation for the very first time. Currently the concept could only be demonstrated on the HDPE plate due to the subtle displacement and the wave speed limited by the camera hardware. The integrated system as well as the algorithms will be optimized and applied to aluminum and composite structures with the high power piezoelectric shaker in the near future. The detailed study for the interaction between the damage and different wave modes will be conducted with full-field three-dimensional measurement using multiple high-speed cameras in the near future. 


\subsection{References}

[5.1] F.G. Yuan, Ed, Structural Health Monitoring (SHM) in Aerospace Structures, Woodhead Publishing, 2016.

[5.2] H.Y. Chang, F.G. Yuan, "Impact damage imaging in a curved composite panel with wavenumber index via Riesz transform," Proc. SPIE, vol.10599, p.105990L, 2018.

[5.3] J. He, C.A. Leckey, P.E. Leser and W.P. Leser, "Multi-mode reverse time migration damage imaging using ultrasonic guided waves," Ultrasonics, vol.94, pp.319-331, 2019.

[5.4] D. Girolamo, H.Y. Chang and F.G. Yuan, "Impact damage visualization in a honeycomb composite panel through laser inspection using zero-lag cross-correlation imaging condition," Ultrasonics, vol. 87, pp.152-165, 2018.

[5.5] Y. Chang and F.G. Yuan, "Damage Detection and Localization using Random Decrement Technique on Metallic Plates,” Proc. of SPIE, International Society for Optics and Photonics, vol. 10970, p. 109702Z, 2019.

[5.6] Y. Chang and F.G. Yuan, "Damage Detection and Isolation via Autocorrelation: A Step toward Passive Sensing," Proc. of SPIE, International Society for Optics and Photonics, vol. 10599, p. 105991K, 2018.

[5.7] C. Grosse and M. Ohtsu, eds., Acoustic emission testing. Springer Science \& Business Media, 2008.

[5.8] P.L. Reu, D.P. Rohe and L.D. Jacobs, "Comparison of DIC and LDV for practical vibration and modal measurements," Mechanical Systems and Signal Processing, vol.86, pp.2-16, 2017. 
[5.9] M. Schaeffer, G. Trainiti and M. Ruzzene, "Optical measurement of in-plane waves in mechanical metamaterials through digital image correlation," Scientific reports, vol.7, p.42437, 2017.

[5.10] A.T. Darnton and M. Ruzzene, "Optical measurement of guided waves," The Journal of the Acoustical Society of America, vol.141, no.5, pp.EL465-EL469, 2017.

[5.11] P.L. Reu, "Speckles and their relationship to the digital camera," Experimental Techniques, vol.38, no.4, pp.1-2, 2014.

[5.12] P.L. Reu, "All about speckles: aliasing," Experimental Techniques, vol.38, no.5, pp.1-3, 2014.

[5.13] P.L. Reu, "All about speckles: contrast," Experimental Techniques, vol.39, no.1, pp.1-2, 2015.

[5.14] H.Y. Chang and F.G. Yuan, "Damage imaging in a stiffened curved composite panel with wavenumber index via Riesz transform," Structural Health Monitoring, 2019, accepted for publication. 


\section{CHAPTER 6 Conclusion and Future Work}

\subsection{Summary and Conclusion}

In this dissertation, the main goal for the entire research is to develop a non-contact camera scanning system as well as a robust damage imaging algorithm to detect, locate and identify the hidden damage in composite structures. Traditionally, the signal is measured by discrete distributed sensors, and for a full-field scanning, the wavefield needs to be reconstructed by measuring each point sequentially. The camera is able to capture the motion at all the points within the scanning region at the same time, which can replace the current laser Doppler vibrometer (LDV) system and enable dealing with transient, non-stationary and non-repeatable problem. However the performance of current high-speed camera system is still limited due to the technology, and both temporal and spatial resolution for measuring the signal on composites are insufficient. In order to develop the damage imaging algorithm, the concept needs to be tested and proved with the current laser system that is mature, stable and reliable.

In the chapter 3, a fully non-contact laser-based NDI system was developed and applied for visualizing a hidden barely visible impact damage (BVID) in a quasi-isotropic [60/0/-60] honeycomb composite panel. The laser system consisted of a Q-switched Nd:YAG pulse laser, a set of galvano-mirrors to rapidly raster scan the Q-switched laser, and a laser Doppler vibrometer (LDV) to measure the ultrasonic wavefield guided. A zero-lag cross correlation (ZLCC) imaging condition as well as a wavenumber-frequency filtering technique were developed to identify and visualize delamination damage in the honeycomb composite panel with this laser system. The ZLCC enabled extraction of momentary standing waves formed and trapped within the delaminated region, in addition to cross-correlating the reflected wavefields from the geometric 
discontinuities (damage boundaries) and the incident guided wavefields. The laser system successfully acquired the guided wavefield in the scanned region and the reconstructed wavefield provided rough information about the damage location. The ZLCC provided accurate location and extent of the impact delamination damage. The image of the delaminated region was in very good agreement with that from an X-ray CT scan. The ZLCC imaging condition also enabled identification of the first two resonant modes moderately accurate accurately for the delaminated area which can be used for characterizing the damage region. The ZLCC operated at higher frequencies can effectively detect minor and smaller defects which may be ignored and hard to be identified.

Since ZLCC was the damage imaging technique based on frequency-wavenumber filtering which requires multiple dimensional Fourier transform, a new imaging condition was needed without complex transform and could be operated in time domain. In chapter4, a new algorithm, named wavenumber index (WI), which was based on image phase derived via Riesz transform (RT) was developed and applied on the guided wave reconstructed by laser system. The new technique was able to identify and visualize the impact damage that occurs in the vicinity of a stiffener, which significantly increases the complexity for analyzing the wavefield. The stiffened curved composite sandwich panel applied with the new method was a section of aileron on a Boeing C-17 Globemaster III military transport aircraft, and it was impacted at NASA Langley Research Center and the impact damage was characterized by an ultrasonic C-scan system for preliminary study and references.

By introducing RT to the guided wave analysis, the un-uniformity and inconsistency of wave intensity can be eliminated, and only the phase information is considered can be of value to increase the sensitivity. The material/geometry discontinuity can be shown by observing the 
change in wave vector. The WI technique integrated all the wave vector values within the sampling time of interest and the wave energy threshold filter to highlight all the material/geometry discontinuities. Because of the high sensitivity in phase information, WI improves the accuracy of damage localization but sacrifice the image quality and signal-to-noise ratio after taking derivative to get the instantaneous wave vector. It also shows consistent damage images for all sensing locations and the directions of wave propagation in comparison with ZLCC. The technique requires no complex wave filtering and mode separation technique, and it is fully employed in time domain, which is computationally efficient and robust.

Wavenumber Index derived via Riesz transform has proven to be a robust and accurate damage imaging condition and has been applied to honeycomb composite and composite aileron scanned by laser system. In chapter 5, the WI method was further modified for the damage visualization on the wavefield reconstructed through the measurement by high-speed digital image correlation (DIC). A major breakthrough on the application of DIC in nondestructive inspection (NDI) and structural health monitoring (SHM) was achieved for capturing guided acoustic wavefield with the aim of damage imaging. The damage was successfully visualized using the scattered guided wavefield measured via high-speed camera with DIC system. By conducting the sampling interleaving and image stitching technique, the integrated system achieved about 250 times of original data requisition rate specified by the camera. Moreover, the experimental parameters and speckle pattern were specifically designed and studied for the best signal quality for measuring sub-micron scale displacements, so that the wavefield sensing became possible. The concept using camera to measure the in-plane wave modes was demonstrated on the soft polymer plate (less than one percent of stiffness of regular CFRP composite structures) in order to introduce large deformation, so that the wavefield of both SH and S0 mode were successfully detected using the 
affordable high-speed camera. The damage imaging condition, wavenumber index (WI), was improved and employed to eliminate ambient noise and misalignment for better accuracy and quality. The algorithms and techniques conducted in this dissertation realized the visualization of damage using WI damage imaging condition via high-speed camera for the very first time in the world.

\subsection{Future Work}

The combination of the developed Wavenumber Index (WI) technique and the high-speed digital image correlation (DIC) system successfully visualized the hidden damage on a polymer plate for the very first time, and the wavefield was reconstructed and shown clearly with the measurement by the camera. The sampling interleaving and image stitching methods were proven to be effective in increasing the equivalent temporal and spatial resolution in guided wave reconstruction. The next step is to extend this technique to the application in composite panels. For composites, in order to visualize the damage, the selection of signal frequency is limited by the wavelength which is corresponded with the damage size, and it will needs a signal frequency in ultrasonic range. The misalignment of sampling interleaving is increased and the signal-to-noise ratio (SNR) is decreased by raising the target signal frequency. Therefore an excitation source with significantly larger magnitude in higher frequency range is require for generating the guided wave for measurement by the camera system, so that the displacement can be above the noise floor of the DIC system and the SNR can be also enhanced.

Since the maximum power output of the current amplifier used for generating the wave signal is only 34 Walt, a more powerful amplifier is required to be compatible with the piezoshaker that can 
generate the signal large enough for composites. These new devices for the experiment in composites are currently in the purchase procedure. This dissertation has shown the potential and possibility of applying the technique onto the composites for the detection, visualization and identification of hidden damages, and the concept can be proven on composites once the new equipment is purchased.

The current Wavenumber Index (WI) has shown good results in the visualization in structures either using laser system or camera system. Riesz transform (RT) provide phase information that is sensitive to all the material/geometrical discontinuities, leading to accurate damage imaging that is consistent to ultrasonic scan and X-ray CT scan. But the WI technique requires taking derivative of the spatial phase, which increases the sensitivity to noise and induces errors for noisy signals. This disadvantage can be improved by employing RT and spatial phase directly without taking derivative, which will need to depend on followers to keep exploring the application of RT in the wavefield analysis. 